ENVIRONMENTAL RESTORATION

\section{PROGRAM}

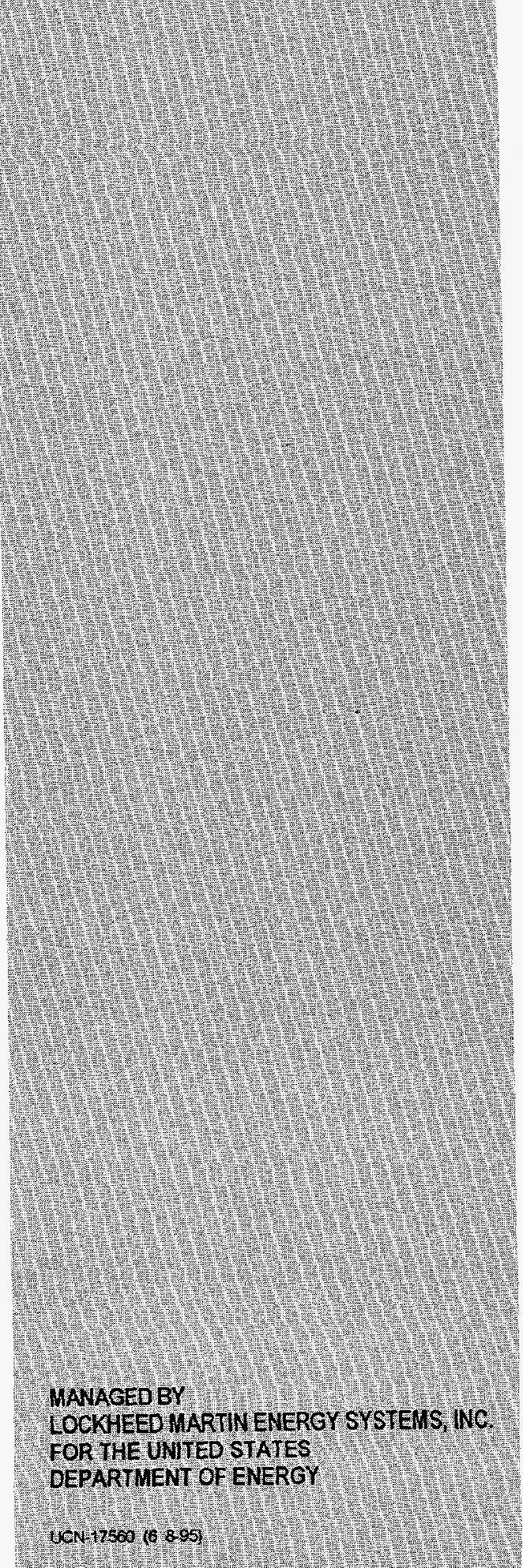

\section{Field Grouting Summary Report on the WAG 4 Seeps 4 and 6 Removal Action Project, Oak Ridge National Laboratory, Oak Ridge, Tennessee}

\section{Volume 2. Appendixes A-D}

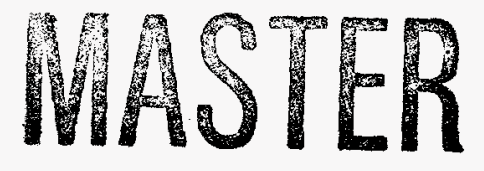

This document has been approved by the ORNL Technical Information Office for release to the public. Date:5/16/97

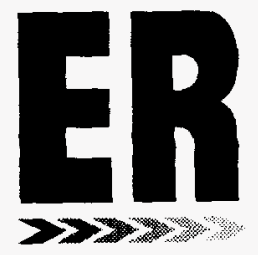




\section{ECO Grouting Specialists Limited}

contributed to the preparation of this document and should not be considered an eligible contractor for its review. 


\section{DISCLAMERR}

Portions of this document may be illegible in electronic image products. Images are produced from the best available original document. 


\section{DISCLAIMER}

This report was prepared as an account of work sponsored by an agency of the United States Government. Neither the United States Government nor any agency thereof, nor any of their employees, make any warranty, express or implied, or assumes any legal liability or responsibility for the accuracy, completeness, or usefulness of any information, apparatus, product, or process disclosed, or represents that its use would not infringe privately owned rights. Reference herein to any specific commercial product, process, or service by trade name, trademark, manufacturer, or otherwise does not necessarily constitute or imply its endorsement, recommendation, or favoring by the United States Government or any agency thereof. The views and opinions of authors expressed herein do not necessarily state or reflect those of the United States Government or any agency thereof. 


\title{
Field Grouting Summary Report on the WAG 4 Seeps 4 and 6 \\ Removal Action Project, Oak Ridge National Laboratory, Oak Ridge, Tennessee
}

\section{Volume 2. Appendixes A-D}

Date Issued-May 1997

\author{
Prepared by \\ ECO Grouting Specialists Limited \\ Cheltenham, Ontario, Canada \\ under subcontract $12 \mathrm{X}-\mathrm{ND} 857 \mathrm{~V}$ \\ Prepared for the \\ U.S. Department of Energy \\ Office of Environmental Management \\ under budget and reporting code EW 20 \\ Environmental Management Activities at the \\ OAK RIDGE NATIONAL LABORATORY \\ Oak Ridge, Tennessee 37831 \\ managed by \\ LOCKHEED MARTIN ENERGY SYSTEMS, INC. \\ for the \\ U.S. DEPARTMENT OF ENERGY \\ under contract DE-AC05-84OR21400
}




\section{EXECUTIVE SUMMARY}

During the summer of 1996, a unique multi-phase, multi-stage, low-pressure permeation grouting pilot program was performed inside portions of four unilned waste disposal trenches at Waste Area Grouping (WAG) 4 at Oak Ridge National I aboratory. (ORNI) in Oak Ridge, Tennessee. The project was deemed a non-time-critical renoval. action under the Comprehensive Environmental Response, Compensation, and Liability Act of 1980 (CERCLA), however, due to a history of heavy precipitation in the fall, the schedule was fast-tracked to meet an October 31, 1996 grouting completion date:

The technical objective of the removal action was to redice the off-site transport of Strontium $90\left({ }^{\circ} \mathrm{S}\right)$ by grouting portions of four waste disposal trenches believed to be responsible for over 70 percent of the ${ }^{2}$ Sr l leaving the site. A goal of the grouting operation was to reduce the average in situ hy draulic conductivity of the grouted waste materials to a value equal to or less than $1 \times 10^{6} \mathrm{~cm} / \mathrm{sec}$. This target hydraulic conductivity value was established to be at least two orders of magnitude lower than that of the surrounding natural ground.

Field grouting activities started in July 1996 and were completed October 21, 1996. Sleeve pipes (driven semi-remotely) were injected at least four times with multiple formulae of regular portland cement based grouts, ultrafine cement based grouts, and acrylamide grouts. Multiple-hole grout injection was monitored using real-time monitoring equipment allowing apparent Lugeen values to be cohtinuously calculated by treating the entire grouting operation as an extended hydraulic conductivity test using grout as the test fluid. By comparing the in situ permeability of the formation to the apparent Lugeon values, the grout formulae were continually adjusted to ensure injection of the grout formulation containing the maximum possible solias content without limiting its ability to permeate the formation. Regular portland and ultrafine eenent based grouts had delayed set times and contained up to cight different ingredients.

Approximately 137,600. gallons of grout were injected. At the completion of production grouting, the effectiveness of the grout spread and the residual in situ hydraulic conductivity of the grouted mass were assessed. The results of hydraulic conductivity tests performed through check pipes after grouting indicate that the average in situ hydraulic conductivity of the waste materals was reduced to a value less than $1 \times 10^{-6}$ $\mathrm{cm} / \mathrm{sec}$. The suecess of this project demonstrates the potential of low pressure permeation grouting as a predictable engineered containment technology for the treatment of low level nuclear waste burial sites.

This report describes brief background to the project describes and analyzes the grouting operations, draws conclusions from the work performed, and presents some of the Tessons learned." 
APPENDIX A

PIPE DRIVING RECORDS 


\begin{tabular}{|c|c|c|c|c|c|c|c|c|c|c|c|c|c|c|c|c|c|c|c|c|c|c|c|c|c|c|c|}
\hline New & Old & Depth & Date & Full & Partial & & & & & & & & $\overline{W S P L}$ & $R F O$ & $O T P E$ & SPE & $\frac{I F C}{1 F C}$ & $\overline{M E A}$ & & & & & & & & Total & \\
\hline Hole No. & Hole No. & Drwen & Drwen & Depth & Depth & $0.1^{\circ}-(-3)(-3)$ & $1-2$ & $2 \cdot 3^{\circ}-(-1)-(10)$ & $3-4^{*}$ & $4.5^{5}$ & 5.6 & $6-7$ & 7.8 & 8. 9 & $|9-10|$ & $\begin{array}{l}10 \cdot- \\
11{ }^{\prime}\end{array}$ & 11. & $\begin{array}{l}12- \\
43 \\
\end{array}$ & $\begin{array}{l}130^{\circ} \\
14^{\prime}\end{array}$ & $\begin{array}{l}14.5^{\circ} \\
15^{\circ}\end{array}$ & $\begin{array}{l}15 . \\
16\end{array}$ & $\begin{array}{l}16- \\
17\end{array}$ & 16 & $\begin{array}{l}180^{\circ} \\
199^{\circ}\end{array}$ & $20^{\circ}$ & Blaws & Comments \\
\hline & & & & & & & & & & & & & & & & & & & & & & & & & & & \\
\hline 1001 & 1008 & 12.3 & 23 July 1996 & $x$ & & & & & & & & & & & & & & & & & & & & & & 0 & \\
\hline 1002 & 1002 & 12.5 & 23 July 1996 & $\mathrm{x}$ & & & & & & & & & & & & & & & & & & & & & & 0 & \\
\hline 1003 & 1003 & 13.8 & 23 July 1996 & $x$ & & & & & & & & & & & & & & & & & & & & & & 0 & . \\
\hline 1004 & 1004 & 14.7 & 23 July 1996 & $x$ & & & & & & & & & & & & & & & & & & & & & & 0 & \\
\hline 1005 & 1005 & 121 & 24 Juty 1996 & $x$ & & & & & & & & & & & & & & & & & & & & & & 0 & \\
\hline 1000 & 1008 & 13.8 & 24 July 1996 & $x$ & & & & & & & & & & & & & & & & & & & & & & 0 & \\
\hline RW107 & RW107 & 10.0 & 7 August 1996 & $x$ & & 4 & 4 & 4 & 6 & 6 & 2 & 2 & 2 & 3 & 3. & & & & & & & & & & & 36 & 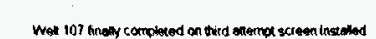 \\
\hline 1008 & 1008 & 140 & 24 Juty 1996 & $x$ & & & & & & & & & & & & & & & & & & & & & & 0 & \\
\hline 1000 & 1009 & 127 & 24 July 1996 & $x$ & & & & & & & & & & & & & & & & & & & & & & 0 & \\
\hline 1010 & 1070 & 140 & 24 Jury 1996 & $x$ & & & & & & & & & & & & & & & & & & & & & & 0 & \\
\hline 1011 & 1011 & 148 & 8 Auruest 1905 & $x$ & & & & & & & & & & & & & & & & & & & & & & 0 & 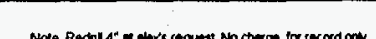 \\
\hline 1012 & 年 & 10 & at initans & $\times$ & & & & & & & & & & & & & & & & & & & & & & a & \\
\hline 1013 & ing & & as & 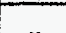 & & & & & & & & & & & & & & & & & & & & & & 列 & \\
\hline & & & w & & & - & & & $M$ & Lent 1 & - & ( & & 烈 & 列 & & & & - & 管 & & & & & & - & \\
\hline & 1014 & 14.8 & 1) August 1996 & $x$ & & 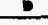 & & & & & & & & $<10$ & 11 & & 3 & 4 & & & & & & & & 59 & ardomio ossosis broms \\
\hline 1018 & 1018 & 14.7 & 1 August 1996 & $x$ & & 4 & 9 & 6 & 8 & 9 & 7 & 10 & 9 & 7 & 12 & 11 & 8 & 7 & 8 & 6 & & & & & & 121 & Eanson \\
\hline 1016 & 1016 & 15.5 & 1 August 1996 & $x$ & & 9 & 10 & $8=$ & & & & 11 & blows & & & $<1$ & 1 & 1 & 1 & 1 & 5 & & & & & 39 & - varysont. \\
\hline 1017 & 1017 & 14.6 & 1 August 1996 & $x$ & & 5 & 6 & 5 & 4 & 4 & 4 & 3 & 3> & 8 & 7 & 6 & 7 & 6 & 9 & $<8$ & & & & & & 74 & Fany son \\
\hline 1018 & 8018 & 138 & 1 Auqust 1996 & $x$ & & 6 & 7 & 6 & 5 & 4 & 9 & 7 & 6 & 12 & 11 & 9 & 14 & 15 & 13 & & & & & & & 124 & 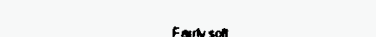 \\
\hline 1040 & 1010 & 148 & I Auguest 1996 & $x$ & & 4 & 5 & 4 & 3 & 6 & 4 & 5 & 5 & 1 & 2 & 2 & 1 & 1 & 3 & 5 & & & & & & 59 & 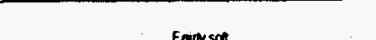 \\
\hline anc & 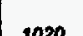 & 130 & 1 Aunust 1906 & $x$ & & 1 & 2 & 3 & 7 & 6 & 9 & $B$ & 7 & 8 & 7 & 9 & 28 & 29 & & & & & & & & 128 & 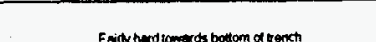 \\
\hline . & (091 & 147 & 1 annust 1006 & $x$ & & 6 & 7 & 3 & 10 & 5 & 6 & 7 & 13 & 17 & 15 & 15 & 14 & 16 & 13 & 17 & & & & & & et 1 & 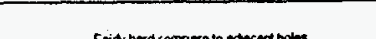 \\
\hline 1029 & 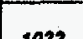 & 100 & 1 aroust 10006 & $x$ & & , & & 14 & plous a & & & & $\Leftrightarrow$ & & 11 & blows & & & & & s & & & & & 䧄 & CH: \\
\hline 1023 & (3) & 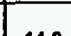 & 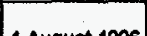 & m & & 2 & 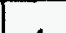 & 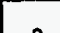 & 6 & 5 & 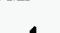 & 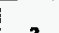 & 7 & 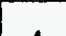 & $\mathrm{A}^{-} \mathrm{C}$ & 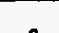 & a & & & & & & & & & $\mathrm{C}_{\mathrm{C}}$ & \\
\hline 1009 & & & & $x$ & & & 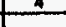 & 2 & & & & & & & & & & 2 & 7 & 6 & & & & & & 67 & Faing son \\
\hline 1024 & 1024 & 14.8 & 1 Auqust 1996 & $\mathbf{x}$ & & 3 & 4 & 2 & 1 & 2 & 3 & 7 & 6 & 1 & 1 & 1 & 2 & 3 & 2 & 6 & & & & & & 44 & son \\
\hline 1025 & 1025 & 14.0 & 1 Auqust 1996 & $x$ & & 3 & 4 & 3 & 2 & 6 & 7 & 3 & 4 & 7 & 6 & 42 & & & & & & & & & & 87 & 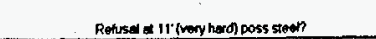 \\
\hline 1026 & 1028 & 14.6 & 1 August 1996 & $x$ & & 4 & 3 & 4 & 5 & 3 & 6 & 2 & 4 & 5 & 3 & 4 & 3 & 1 & 2 & 6 & & & & & & 55 & Fains sot \\
\hline 1027 & 1027 & 13.7 & 1 Auqust 1996 & $x$ & & 4 & 3 & 5 & 4 & 5 & 2 & 1 & 2 & 3 & 2 & 1 & 1 & 2 & 4 & & & & & & & 39 & Forin son \\
\hline 2000 & 1000 & 140 & 1 Anoust 1906 & $x$ & & 3 & 4 & 4 & 2 & 2 & 1 & 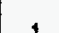 & 6 & 7 & 11 & 13 & 14 & 17 & 29 & & & & & & & $m_{0}$ & 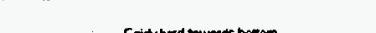 \\
\hline
\end{tabular}




\begin{tabular}{|c|c|c|c|c|c|c|c|c|c|c|c|c|c|c|c|c|c|c|c|c|c|c|c|c|c|c|c|}
\hline New & Old & Depth & Date & Full & Pantial & & & & & & & $B L O$ & WSP & $R F$ & OT PE & $\frac{S P E}{10 .}$ & IFIC & $\frac{1 E A}{12 .}$ & $\frac{R E}{13-}$ & & & & & & & Total & \\
\hline Hole No. & Hole No. & Diven & Diven & Depth & Depth & $0 . y^{\prime}$ & 1.2 & $2-3^{\prime}$ & $3-4$ & 4.5 & $5-6$ & $6 \cdot 7$ & 7.8 & $8-9$ & $9-10$ & $\begin{array}{l}10 . \\
111^{\circ}\end{array}$ & $112^{11}$ & $\begin{array}{r}12 \cdot \\
130^{\prime}\end{array}$ & $\begin{array}{l}13-4^{\circ} \\
14^{\circ}\end{array}$ & $\begin{array}{l}14- \\
15 \\
15\end{array}$ & $\begin{array}{l}15- \\
16\end{array}$ & $\begin{array}{l}16- \\
17 \\
\end{array}$ & 16 & $\begin{array}{l}10- \\
19^{\prime} \\
\end{array}$ & 20 & Blowe & Comments \\
\hline & & & & & & & & & & & & & 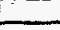 & 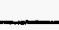 & & & & & & & & & & & & & \\
\hline 1020 & 1020 & 14.7 & 1 August 1996 & $x$ & & 4 & 3 & 4 & 6 & 3 & 3 & 4 & 5 & 7 & 11 & 14 & 10 & 9 & 13 & 7 & & & & & & 105 & 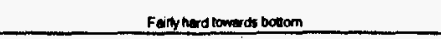 \\
\hline 1030 & 1030 & 14.7 & August 1996 & $x$ & & 3 & 4 & 6 & 4 & 5 & 3 & 4 & 5 & 4 & 6 & 2 & 7 & 3 & 4 & 7 & & & & & & 67 & Fonty ent \\
\hline 1031 & 1031 & 13.2 & 1 August 1996 & $x$ & & 3 & 3 & 4 & 2 & 2 & 4 & 8 & 3 & 5 & 7 & 9 & 30 & 21 & 23 & & & & & & & 124 & Foring sor \\
\hline 1032 & 1032 & 14.3 & 1 August 1996 & $x$ & & 3 & 2 & 2 & 1 & 2 & 2 & 2 & 1 & 1 & 1 & 3 & 6 & 5 & 3 & 4 & & & & & & 38 & veryson \\
\hline 8033 & 1033 & 147 & 1 August 1996 & $\mathrm{x}$ & & 3 & 4 & 1 & 1 & 4 & 3 & 4 & 4 & 2 & 4 & 4 & 4 & 4 & 3 & 4 & & & & & & 51 & vorsson. \\
\hline 1034 & 1034 & 14.7 & 5 August 1996 & $\mathrm{x}$ & & 2 & 5 & 4 & 5 & 4 & 4 & 2 & 2 & 2 & 2 & 3 & 1 & 1 & 1 & 1 & & & & & & 39 & 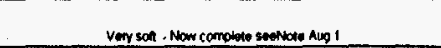 \\
\hline 1035 & 1035 & 145 & 1 August 1996 & $x$ & & 2 & 6 & 6 & 3 & 3 & 3 & 2 & 1 & 2 & 2 & 7 & 10 & 10 & 35 & 34 & & & & & & 126 & form $\operatorname{sen}$ \\
\hline 1036 & 1038 & 14.3 & 1 August 1996 & $x$ & & 2 & 5 & 4 & 3 & 2 & 2 & 1 & 1 & 2 & 2 & 3 & 2 & 3 & 2 & 5 & & & & & & 39 & Vem sont \\
\hline 1037 & 1037 & 143 & 1 Auqust 1996 & $x$ & & 2 & 3 & 4 & 4 & 4 & 4 & 3 & 4 & 4 & 3 & 3 & 2 & 2 & 2 & & & & & & & 44 & \\
\hline 1038 & 1038 & 133 & 5 Aurust 1995 & $x$ & & 3 & 4 & 5 & 5 & 5 & 5 & 4 & 4 & 5 & 5 & 5 & 6 & 7 & 8 & & & & & & & 71 & menotsen \\
\hline 1038 & 1039 & 14.2 > & 5 Ay & $x$ & & 5 & 6 & 7 & 4 & 3 & 4 & 3 & 3 & 3 & 3 & 2 & 3 & 3 & 3 & 30 & & & & & & $B 2$ & fantron \\
\hline 1040 & 1040 & 113 & 5 Auqust 1996 & $x$ & & 5 & 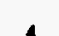 & 4 & 3 & 3 & 3 & 4 & 4 & 5 & 6 & 28 & 77 & & & & & & & & & 96 & s \\
\hline 1081 & 1011 & $\frac{11.3}{11.3}$ & 5 August 1996 & $\frac{\hat{x}}{x}$ & & $\frac{5}{7}$ & 8 & 8 & $\frac{5}{6}$ & $\frac{5}{5}$ & $\frac{5}{3}$ & 2 & 6 & 20 & 28 & 30 & 32 & & & & & & & & & 155 & \\
\hline 1002 & 1042 & 11.3 & 5 August 1996 & $\bar{x}$ & & 6 & 7 & 6 & 6 & 5 & 4 & 3 & 6 & 8 & 18 & 25 & 32 & & & & & & & & & 126 & \\
\hline 1043 & 1043 & 14.2 & 5 August 1996 & $x$ & & 3 & 4 & 4 & 4 & 4 & 4 & 4 & 6 & 8 & 13 & 14 & 8 & 6 & 10 & 19 & & & & & & 111 & \\
\hline ram & $10 \mathrm{~m}$ & 14.9 & 5 August 1996 & $x$ & & 8 & 8 & 8 & 12 & 6 & 1 & 4 & 3 & 3 & 2 & 2 & 2 & 3 & 3 & 21 & & & & & & 89 & \\
\hline 1045 & 1006 & 16.4 & 5 August 1996 & $x$ & & 7 & 5 & 5 & 4 & 1 & 3 & 2 & 5 & 6 & 1 & 7 & 8 & 8 & 9 & 12 & 20 & 26 & & & & 138 & 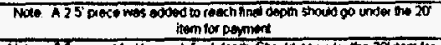 \\
\hline 1048 & 1048 & 16.3 & 5 Auguat 1996 & $x$ & & 4 & 4 & 4 & 4 & 1 & 3 & 3 & 3 & 5 & 5 & 5 & 5 & 6 & 6 & 6 & 6 & 20 & & & & 93 & 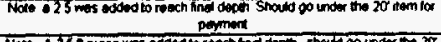 \\
\hline 1047 & 1047 & 16.3 & 5 August 1996 & $x$ & & 4 & 4 & 4 & 5 & 4 & 3 & 3 & 3 & 2 & 2 & 3 & 4 & 4 & 4 & 4 & 8 & 12 & & & & 71 & 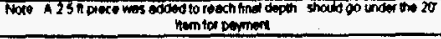 \\
\hline 1040 & 1040 & 16.5 & 5Auguset 1996 & $\bar{x}$ & & 4 & 5 & 3 & 3 & 5 & 6 & 6 & 6 & 8 & 9 & 12 & 25 & 27 & 14 & 19 & 32 & 30 & & & & 218 & 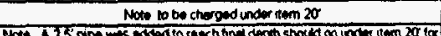 \\
\hline 1040 & 1000 & 16.5 & 5 August 1996 & $x$ & & 4 & 4 & 4 & 4 & 3 & 3 & 3 & 2 & $\underline{6}$ & 8 & 3 & 2 & 2 & 2 & 20 & 20 & 25 & & & & & Dis \\
\hline 1050 & 1050 & 16.7 & 5 A August 1996 & $\underline{x}$ & & 11 & 11 & 11 & 11 & 11 & 11 & 11 & 11 & 11 & 11. & 11 & 4 & 4 & 4 & 4 & 4 & 21 & & & & 162 & 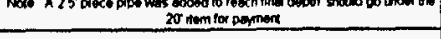 \\
\hline 1034 & 1051 & 14.9 & S August 1996 & $\underline{x}$ & & 1 & 4 & 4 & 4 & 3 & 3 & 3 & 3 & 4 & 3 & 3 & 4 & 8 & 6. & 4 & & & & & & 60 & \\
\hline 1052 & 1052 & 14.3 & 5 August 1996 & $x$ & & 8 & 10 & 10 & 8 & 6 & 6 & 3 & 5 & 5 & 4 & 4 & 4 & 4 & 4 & 14 & & & & & & 97 & \\
\hline 1053 & 1053 & 14.6 & 5 August 1996 & $x$ & & 10 & 6 & 6 & 6 & 6 & 7 & 8 & 8 & 8 & 6 & 4 & 4 & 4 & 4 & 14 & & & & & & 101 & \\
\hline 1054 & 1054 & 14.8 & 5 August 1996 & $x$ & & 4 & 4 & 4 & 4 & 4 & 6 & 6 & 6 & 6 & 4 & 4 & 4 & 4 & 4 & 4 & 17 & & & & & 85 & \\
\hline 1056 & 1055 & 15.0 & 5 August 1996 & $x$ & & 6 & 6 & 6 & 7 & 7 & 7 & 7 & 6 & 6 & 6 & 7 & 7 & 7 & 7 & 17 & & & & & & 109 & \\
\hline 1056 & 1056 & 1.5 & 5 August 1996 & & $x$ & & & & & & & & & & & & & & & & & & & & & & \\
\hline R1056 & $R 1056$ & 14.9 & 7 August 1996 & $x$ & & 5 & 5 & 6 & 6 & $\theta^{\circ}+$ & 6 & 5 & 6 & 5 & 6 & 5 & 5 & 5 & 6 & 16 & & & & & & 93 & Retessed of id if \\
\hline$\frac{-1050}{10.57}$ & $\frac{\pi}{1057}$ & $\frac{19.0}{15.0}$ & 7 August 1996 & $x$ & & 6 & 8 & 8 & 10 & 80 & 30 & 10 & 10 & 7 & 10 & 10 & 15 & 15 & 17 & 17 & & & & & & 253 & 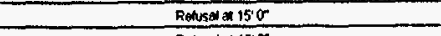 \\
\hline 1058 & 1058 & 15.0 & 7 Auqust 1996 & $x$ & & 4 & 6 & 8 & 16 & 30 & 5 & 5 & 5 & $\frac{4}{7}$ & 4 & 4 & 5 & 6 & 6 & $\frac{16}{16}$ & & & - & & & 124 & 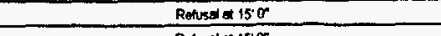 \\
\hline 1059 & 1059 & 15.0 & 7 August 1996 & $\bar{x}$ & & 4 & 4 & $a$ & 5 & 8 & 12 & 8 & 6 & 1 & 5 & 5 & 5 & 4 & 4 & 15 & & & & & & 96 & Retusadeat is' or \\
\hline 1000 & 1000 & 14.9 & 7 August 1996 & $x$ & & 3 & 5 & 7 & 14 & 12 & 8 & 6 & 5 & 6 & 6 & 9 & 9 & 12 & 15 & 18 & & & & & & 137 & 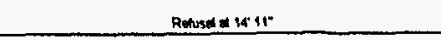 \\
\hline 1081 & 1081 & 149 & 6 August 1996 & $x$ & & 6 & 5 & 5 & 4 & 1 & 4 & 4 & 3 & 4 & 4 & 5 & 5 & 5 & 5 & 15 & & & & & & 78 & \\
\hline 1062 & 1062 & 15.0 & 6 August 1996 & $\bar{x}$ & & 5 & 6 & 6 & 6 & 6 & 6 & 6 & 5 & 5 & 6 & 7 & 7 & 7 & 9 & 16 & & & & & & 103 & \\
\hline 1063 & 1063 & 15.0 & 6 August 1996 & $\bar{x}$ & & 8 & 8 & 8 & 7 & 6 & 5 & 4 & 3 & 5 & 7 & 7 & 8 & 10 & 12 & 13 & & & & & & 111 & \\
\hline & 1084 & 15.2 & 7 August 1996 & $x$ & & 5 & 5 & 5 & 5 & 5 & 5 & 5 & 5 & 6 & 8 & 12 & 8 & 7 & 6 & 5 & 12 & & & & & 104 & conchesed \\
\hline
\end{tabular}




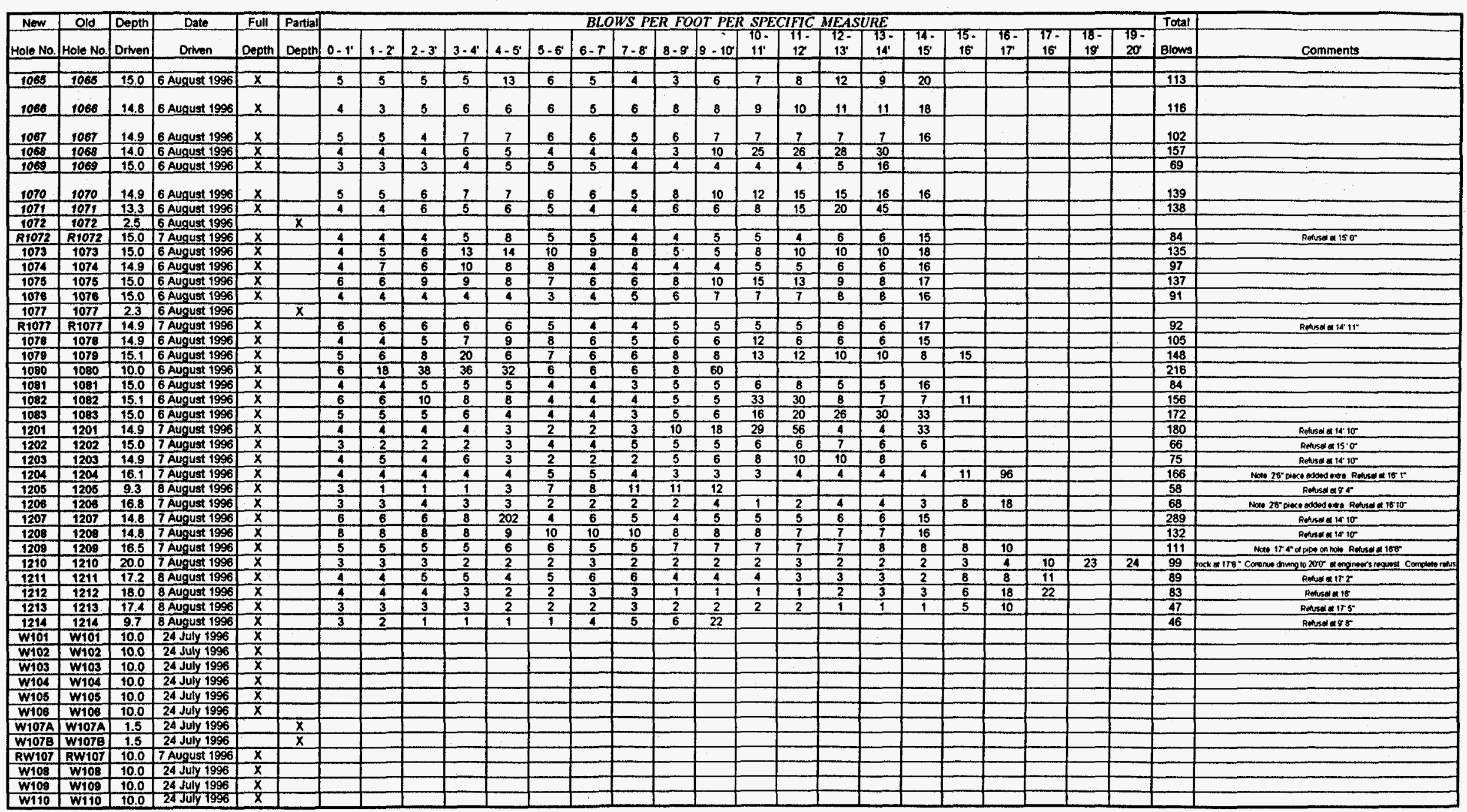




\begin{tabular}{|c|c|c|c|c|c|c|c|c|c|c|c|c|c|c|c|c|c|c|c|c|c|c|c|c|c|c|c|}
\hline New & Old & Depth & Dale & Full & Partial & & & & & & & & BLO & S PER & FOOT PE & RPPEC & FIC MEA & ASURE & & & & & & & & \begin{tabular}{|l} 
Total \\
\end{tabular} & \\
\hline & Hole No. & $\begin{array}{c}\text { Driven } \\
(\mathrm{i})\end{array}$ & Driven & Depth & Depth & $0.1^{\prime}$ & 1.2 & $2.3^{\circ}$ & $3-4^{\prime}$ & $4-5$ & 5.6 & 6.7 & $7.8^{\circ}$ & $8 \cdot 9$ & $9-10$ & $10-11$ & $11 \cdot 12$ & $12 \cdot 13^{\prime}$ & $13-14^{\prime}$ & $14-15^{\prime}$ & $15 \cdot 16^{6}$ & $16-17$ & $17-16^{\prime}$ & $18-19$ & $19 \cdot 20^{\circ}$ & Blows & Comments \\
\hline & & & & & & & & & & & & & & & & & & & & & & & & & & & \\
\hline 2001 & 2001 & 13.6 & 22 July 1996 & $\bar{x}$ & & 3 & & 2 & & 1 & & 1 & & 1 & & 2 & & 7 & 12 & & & & & & & $\frac{29}{20}$ & \\
\hline 2002 & 2002 & 14.3 & 22 July 1996 & $x$ & & 2 & & 2 & & 1 & & 1 & & 1 & & 3 & 3 & 4 & 7 & 11 & & & & & & 35 & \\
\hline 2003 & 2003 & 14.3 & 22 July 1996 & $x$ & & 2 & & 2 & & 3 & & 1 & & 1 & & 3 & $\frac{6}{9}$ & 11 & $\frac{13}{2}$ & & & & & & & $\frac{42}{26}$ & \\
\hline 2004 & 2004 & 15.3 & 22 July 1996 & $x$ & & 3 & & 2 & & 2 & & 1 & & 1 & & 1 & 3 & 4 & 3 & 6 & & & & & & 26 & \\
\hline 2005 & 2005 & 14.7 & 13 August 1996 & $x$ & & 0 & 0 & 0 & 0 & 0 & 0 & 0 & 1 & 1 & 1 & 1 & 1 & 1 & 6 & 14 & & & & & & 26 & 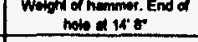 \\
\hline 2006 & 2006 & 14.5 & 23 July 1996 & $x$ & & 2 & 3 & 4 & 6 & 5 & 7 & 8 & 11 & 6 & 5 & 7 & 11 & 13 & 6 & 11 & & & & & & 105 & \\
\hline 2007 & 2007 & 15.3 & 23 July 1996 & $\bar{x}$ & & 1 & 2 & 3 & 5 & 4 & 6 & 8 & 9 & 10 & 3 & 4 & 2 & 11 & 6 & 11 & 14 & & & & & 99 & \\
\hline 2008 & 2008 & 13.1 & 23 July 1996 & $\bar{x}$ & & & & & & & & & & & & & & & & & & & & & & 0 & \\
\hline 2008 & 2009 & 14.2 & 13 August 1996 & $x$ & & 0 & 0 & 0 & 0 & 0 & 0 & 0 & 0 & 0 & 1 & 1 & 1 & 1 & 4 & 6 & & & & & & 14 & 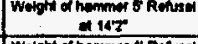 \\
\hline 2010 & 2010 & 15.4 & 13 Auqust 1996 & $x$ & & 0 & 0 & 0 & 0 & 0 & 0 & 0 & 0 & 0 & 1 & 1 & 1 & 2 & 3 & 6 & 14 & & & & & 28 & 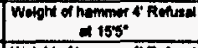 \\
\hline 2011 & 2011 & 14.8 & 13 Auqust 1996 & $x$ & & 0 & 0 & 0 & 0 & 0 & 0 & 0 & 1 & 1 & 4 & 5 & 6 & 8 & 18 & 51 & & & & & & 94 & 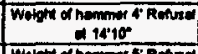 \\
\hline 2012 & 2012 & 14.8 & 13 August 1996 & $x$ & & 1 & 1 & 0 & 0 & 0 & 0 & 0 & 0 & 1 & 1 & 1 & 2 & 2 & 6 & 41 & & & & & & 56 & 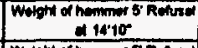 \\
\hline 2013 & 2013 & 14.5 & 13 August 1996 & $x$ & & 0 & 0 & 0 & 0 & 0 & 0 & 0 & 0 & 0 & 1 & 1 & 1 & 2 & 3 & 12 & & & & & & 20 & 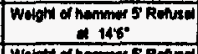 \\
\hline 2014 & 2014 & 14.7 & 13 Auqust 1996 & $x$ & & 0 & 0 & 0 & 0 & 0 & 0 & 0 & 0 & 1 & 1 & 2 & 2 & 2 & 4 & 22 & & & & & & 34 & 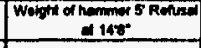 \\
\hline 2015 & 2015 & 21.5 & 13 August 1996 & $x$ & & 0 & 0 & 0 & 0 & 0 & 0 & 0 & 0 & 0 & 1 & 2 & 2 & 3 & 4 & 4 & 3 & 3 & 3 & 3 & \begin{tabular}{|c|c|}
$17+16+1$ \\
9
\end{tabular} & $\cdots 80$ & 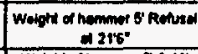 \\
\hline 2016 & 2016 & 16.1 & 13 Auqust 1996 & $x$ & & 0 & 0 & 0 & 0 & 0 & 0 & 0 & 0 & 0 & 1 & 0 & 0 & 0 & 0 & 0 & 1 & 15 & & & & 17 & 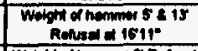 \\
\hline 2017 & 2017 & 14.6 & 13 Auqust 1996 & $x$ & & 0 & 0 & 0 & 0 & 0 & 0 & 0 & 0 & 0 & 0 & 1 & 1 & 1 & 4 & 16 & & & & & & 23 & 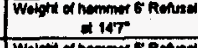 \\
\hline 2018 & 2018 & 15.5 & 13 Auqust 1996 & $x$ & & 0 & 0 & 0 & 0 & 0 & 0 & 0 & 1 & 1 & 1 & 1 & 1 & 1 & 1 & 1 & 8 & & & & & 16 & 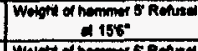 \\
\hline 2010 & 2019 & 15.0 & 13 August 1996 & $x$ & & 0 & 0 & 0 & 0 & 0 & 0 & 0 & 0 & 1 & 2 & 4 & 4 & 6 & 6 & 16 & & & & & & 39 & 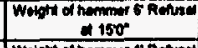 \\
\hline 2020 & 2020 & 15.0 & 13 Auqust 1996 & $x$ & & 0 & 0 & 0 & 0 & 0 & 0 & 0 & 2. & 2 & 3 & 3 & 4 & 6 & 6 & 6 & & & & & & 32 & 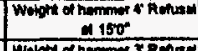 \\
\hline 2021 & 2021 & 15.4 & 13 August 1996 & $x$ & & 0 & 0 & 0 & 0 & 0 & 0 & 2 & 1 & 1 & 1 & 1 & 1 & 1 & 1 & 1 & 8 & & & & & 18 & 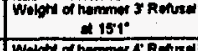 \\
\hline 2022 & 2022 & 15.8 & 13 Auqust 1996 & $x$ & & 0 & 0 & 0 & 0 & 0 & 0 & 0 & 0 & 2 & 1 & 1 & 1 & 1 & 1 & 1 & 8 & & & & & 16 & 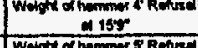 \\
\hline 2023 & 2023 & 15.5 & 13 Auqust 1996 & $x$ & & 0 & 0 & 0 & 0 & 0 & 0 & 0 & 0 & 0 & 2 & 1 & 1 & 1 & 2 & 20 & & & & & & 27 & 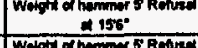 \\
\hline 2024 & 2024 & 16.0 & 13 Auqust 1996 & $x$ & & 0 & 0 & 0 & 0 & 0 & 0 & 0 & 0 & 2 & 1 & 1 & 1 & 1 & 1 & 1 & 30 & & & & & 38 & 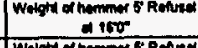 \\
\hline 2025 & 2025 & 15.0 & 13 Auqust 1996 & $\underline{x}$ & & 0 & 0 & 0 & $\underline{0}$ & 0 & 0 & 0 & 0 & 60 & 208 & & & & & & & & & & & 268 & 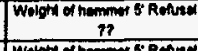 \\
\hline 2026 & 2027 & 15.0 & 13 August 1996 & $x$ & & 0 & 0 & 0 & 0 & 0 & 0 & 0 & 0 & 1 & 1 & 2 & 4 & 10 & 15 & 16 & & & & & & 49 & 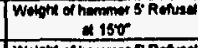 \\
\hline 2027 & 2028 & 16.8 & 13 Auqust 1996 & $x$ & & 0 & 0 & 0 & 0 & 0 & 0 & 0 & 0 & 0 & 1 & 1 & 1 & 1 & 1 & 1 & 15 & 17 & & & & 38 & 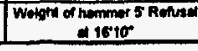 \\
\hline
\end{tabular}




\begin{tabular}{|c|c|c|c|c|c|c|c|c|c|c|c|c|c|c|c|c|c|c|c|c|c|c|c|c|c|c|c|}
\hline New & Old & Depth & Date & Full & Pantial & & & & & & & & BLO & S PER & $F O O T P E$ & ER SPEC & IFIC MEA & SURE & & & & & & & & Total & \\
\hline Hole No. & Hole No. & (A) & Driven & Depth & Depth & 0.1 & 1.2 & $2-3^{\prime}$ & $3-4^{\prime}$ & 4-5' & $5-6$ & $6-7$ & $7.8^{\prime}$ & $8-9$ & $9-10^{\prime}$ & $10 \cdot 11^{\prime}$ & $11-12$ & $12-13^{\prime}$ & $13-14$ & $14-15^{\prime}$ & $15-16^{\prime}$ & $16-17$ & $17-16$ & $18-19^{\prime}$ & $19-20$ & Blows & Comments \\
\hline 2028 & 2029 & 17.1 & 13 August 1996 & $x$ & & 0 & 0 & 0 & 0 & 0 & 0 & 0 & 0 & 1 & 1 & 1 & 1 & 1 & 1 & 1 & 14 & 26 & 20 & & & 67 & 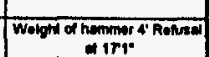 \\
\hline 2029 & 2030 & 16.5 & 13 August 1996 & $x$ & & 0 & 0 & 0 & 0 & 0 & 0 & 0 & 0 & 0 & 0 & 0 & 0 & 0 & 1 & 1 & 6 & 18 & & & & 26 & 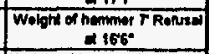 \\
\hline 2030 & 2031 & 9.0 & 14 Auqust 1996 & $x$ & & 0 & 1 & 1 & 0 & 3 & 3 & 4 & 120 & 125 & & & & & & & & & & & & 257 & 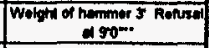 \\
\hline 2031 & 2032 & 17.0 & 13 August 1996 & $x$ & & & & & & & & & & & & & & & & & & & & & & 0 & \\
\hline 2032 & 2033 & 17.3 & 14 August 1996 & $x$ & & 0 & 0 & 0 & 0 & 0 & 0 & 0 & 0 & 2 & 2 & 2 & 135 & 18 & 2 & 2 & 14 & 30 & 35 & & & 242 & 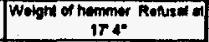 \\
\hline 2033 & 2036 & 17.5 & 8 August 1996 & $x$ & & 3 & 3 & 4 & 3 & 3 & 3 & 2 & 2 & 2 & 2 & 2 & 3 & 2 & 2 & 2 & 4 & 18 & 18 & & & 75 & Renseden irs: \\
\hline 2034 & 2037 & 15.8 & 8 Auqust 1996 & $\bar{x}$ & & 4 & 3 & 3 & 3 & 3 & 3 & 3 & 3 & 5 & 10 & 12 & 15 & 7 & 7 & 7 & 20 & & & & & 108 & Reneded a 1510 \\
\hline 2035 & 2038 & 17.5 & 8 Auqust 1996 & $x$ & & 1 & 1 & 1 & 1 & 1 & 2 & 2 & 2 & 1 & 1 & 1 & 1 & 2 & 6 & 6 & 8 & 8 & 30 & & & 75 & Refunecteiro \\
\hline 2036 & 2039 & 20.0 & 8 August 1996 & $\frac{x}{x}$ & & 4 & 4 & 4 & 6 & 8 & 16 & 30 & 18 & 4 & 4 & 4 & 4 & 4 & 4 & 4 & 3 & 3 & 3 & 3 & 3 & 133 & Nat on sontom $y$ yat in 20 \\
\hline 2037 & 2010 & 14.7 & 8 August 1996 & $x$ & & 4 & 4 & 6 & 4 & 4 & 4 & 4 & 3 & 3 & 4 & 6 & 5 & 5 & 7 & 7 & & & & & & .70 & folused of w:F \\
\hline 2038 & 2041 & 20.0 & 8 August 1996 & $x$ & & 4 & 4 & 4 & 2 & 2 & 3 & 3 & 2 & 2 & 2 & 2 & 1 & 1 & 1 & 1 & 1 & 2 & 20 & 13 & 7 & 77 & Ronuset a 2000 \\
\hline 2039 & 2042 & 15.0 & 9 August 1996 & $x$ & & 3 & 3 & 3 & 4 & 4 & 3 & 3 & 3 & 3 & 4 & 5 & 6 & 7 & 7 & 10 & & & & & & 68 & Reolusener $150^{\circ}$ \\
\hline 2040 & 2043 & 20.0 & 9 August 1996 & $x$ & & 3 & 3 & 4 & 4 & 1 & 1 & 1 & 2 & 1 & 1 & 1 & 1 & 1 & 1 & 2 & 1 & 1 & 15 & 17 & 19 & 80 & Rensara $=200^{\circ}$ \\
\hline 2041 & 2044 & 19.3 & 9 August 1996 & $x$ & & 2 & 3 & 3 & 3 & 2 & 1 & 1 & 1 & 1 & 1 & 1 & 2 & 5 & 2 & 1 & 18 & 53 & & & & 100 & Ronsen I I $199^{\circ}$ \\
\hline 2042 & 2045 & 14.8 & 9 August 1996 & $\bar{x}$ & & 3 & 3 & 3 & 3 & 3 & 3 & 4 & 4 & 4 & 4 & 4 & 5 & 5 & 5 & 16 & & & & & & 69 & Relused in if io \\
\hline 2043 & 2046 & 15.1 & 9 August 1996 & $\bar{x}$ & & 4 & 5 & 8 & 3 & 3 & 4 & 4 & 4 & 4 & 4 & 4 & 4 & 5 & 5 & 6 & & & & & & 67 & Renesein $150^{\circ}$ \\
\hline$A$ & 20,7 & 3.0 & 9 August 1996 & & & & & & & & & & & & & & & & & & & & & & & & \\
\hline 204 & $R 2047$ & 15.0 & 9 August 1996 & $x$ & $\bar{x}$ & 2 & 4 & 4 & 3 & 3 & 3 & 3 & 3 & 5 & 5 & 6 & 5 & 5 & 5 & 11 & & & & & & 67 & Renusen a is 0 \\
\hline 2045 & 2048 & 20.0 & 9August 1996 & & & 2 & 4 & 12 & 2 & 2 & 2 & 3 & $\frac{2}{2}$ & 2 & 1 & 1 & 1 & 2 & 2 & 2 & 5 & 6 & 7 & 7 & 11 & 76 & Renrea $\times 20^{\circ}$ \\
\hline 2046 & 2049 & 15.0 & 9 August 1996 & $\bar{x}$ & & 2 & 1 & 1 & 2 & 2 & 2 & 2 & 2 & 3 & 4 & 4 & 5 & 5 & 5 & 9 & & & & & & 49 & Aonuer e e 15: $0^{\circ}$ \\
\hline $20 \sqrt{7}$ & 2050 & 15.0 & 9 August 1996 & $x$ & & 2 & 1 & 1 & 2 & 2 & 2 & 2 & 2 & 3 & 4 & 4 & 5 & 5 & 5 & 5 & & & & & & 45 & Renzed in 180 \\
\hline W201 & W201 & 10.0 & 22 Julv 1996 & $\frac{x}{x}$ & & & & & & & & & & & & & & & & & & & & & & & \\
\hline W202 & $w_{202}$ & 5.0 & 22 July 1996 & $x$ & & & & & & & & & & & & & & & & & & & & & & & \\
\hline W203 & W203 & 10.0 & 22 July 1996 & $x$ & & & & & & & & & & & & & & & & & & & & & & & \\
\hline W204 & \begin{tabular}{|l|} 
W204 \\
\end{tabular} & 10.0 & 22 July 1996 & & $x$ & & & & & & & & & & & & & & & & & & & & & 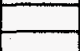 & \\
\hline W205 & W205 & 10.0 & 22 July 4996 & $x$ & & & & & & & & & & & & & & & & & & & & & & & \\
\hline W2026 & W2026 & 10.0 & 13 August 1996 & $x$ & & 0 & 0 & 0 & 0 & 0 & 0 & 0 & 2 & 1 & 1 & & & & & & & & & & & 4 & 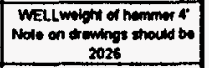 \\
\hline 01 & D1 & 10.0 & 9 August 1996 & $\underline{x}$ & & 2 & 3 & 3 & 3 & 3 & 3 & 3 & 3 & 3 & 3 & & & & & & & & & & & 29 & 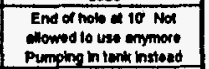 \\
\hline D2 & D2 & 10.0 & 9 August 1996 & $x$ & & 2 & 2 & 2. & 2 & 2 & 2 & 2 & 2 & 2 & 2 & & & & & & & & & & & 20 & 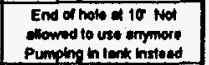 \\
\hline D3 & D & 4.0 & 9 Aucust 1996 & & $\underline{x}$ & 4 & 4 & 4 & 8 & & & & & & & & & & & & & & & & & 20 & 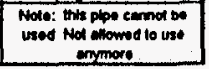 \\
\hline
\end{tabular}




\begin{tabular}{|c|c|c|c|c|c|c|c|c|c|c|c|c|c|c|c|c|c|c|c|c|c|c|c|c|c|c|c|}
\hline New & Old & Depth & Date & Full & Partial & \multicolumn{20}{|c|}{ BLOWS PER FOOT PER SPECIFIC MEASURE } & \multirow{2}{*}{\begin{tabular}{|c|} 
Tolit \\
Blows \\
\end{tabular}} & \multirow[b]{2}{*}{ Comments } \\
\hline Hole No. & Hole No. & Driven & Driven & Depth & Depth & $0.1^{\prime}$ & $1-2$ & 2-3 & $3.4^{\circ}$ & 4.5 & $5-6$ & 6.7 & $7-8^{\prime}$ & $8-9$ & $9-10$ & $10-11$ & $11-12$ & $12-13$ & $13-14$ & $14-15$ & $15-16$ & $|16-17|$ & $17 \cdot 16$ & $18 \cdot 19$ & $19-20$ & & \\
\hline 3001 & $3 M$ & 16.7 & 30 Auoust 1996 & $\bar{x}$ & & 20 & 17 & 6 & & & & & & & 1 & 2 & 2 & 3 & & 5 & & & & & & & \\
\hline 3002 & 3006 & 15.0 & 30 August 1996 & $x$ & & $\frac{07}{16}$ & 8 & $\frac{6}{6}$ & $\frac{6}{4}$ & $\frac{5}{4}$ & $\frac{3}{4}$ & $\frac{3}{2}$ & $\frac{3}{1}$ & $\frac{2}{2}$ & $\frac{1}{2}$ & $\frac{2}{1}$ & 2 & $\frac{3}{2}$ & $\frac{4}{10}$ & $\frac{2}{17}$ & 5 & 8 & & & & 95 & Retused 10 108 \\
\hline 3003 & $3 L$ & 15.7 & 30 August 1996 & $\hat{x}$ & & 17 & 12 & 5 & $\frac{7}{5}$ & $\frac{4}{5}$ & $\frac{4}{3}$ & $\frac{2}{1}$ & $\frac{1}{2}$ & $\frac{2}{4}$ & $\frac{5}{6}$ & 5 & $-\frac{1}{4}$ & $\frac{2}{4}$ & $\frac{10}{12}$ & $\frac{117}{17}$ & 22 & & & & & 80 & 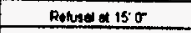 \\
\hline 3004 & 3009 & 14.8 & 30 August 1996 & $\bar{x}$ & & 20 & 8 & $\frac{2}{2}$ & $\frac{2}{2}$ & $\frac{\pi}{2}$ & $\frac{5}{2}$ & $\frac{1}{2}$ & $\frac{6}{2}$ & 1 & $\frac{5}{3}$ & $\frac{5}{2}$ & $\frac{4}{2}$ & $\frac{4}{2}$ & $\frac{12}{12}$ & $\frac{11}{20}$ & 22 & & & & & 124 & Retssa e ist \\
\hline 3005 & $3 K$ & 14.6 & 30 August 1996 & $x$ & & 12 & 10 & 8 & $\frac{6}{5}$ & 4 & $\frac{2}{5}$ & $\frac{4}{1}$ & $\frac{6}{1}$ & $\frac{1}{2}$ & $\frac{\pi}{4}$ & $\frac{\pi}{4}$ & 4 & $\frac{3}{4}$ & $\frac{12}{6}$ & $\frac{20}{15}$ & & & & & & 82 & Rethed at 14:10 \\
\hline 3006 & 3012 & 12.3 & 30 August 1996 & $\frac{x}{x}$ & & 10 & $\frac{10}{6}$ & $\frac{\pi}{4}$ & $\frac{3}{3}$ & 3 & 3 & 2 & $\frac{\pi}{2}$ & 4 & 4 & 12 & 20 & & & & & & & & & 85 & Bensogat at \\
\hline 3007 & 3013 & 15.8 & 30 August 1996 & $x$ & & 24 & 12 & 6 & 5 & 4 & 4 & 4 & 1 & 1 & $-\frac{7}{2}$ & $\frac{12}{2}$ & & 2 & $\sqrt{2}$ & 2 & 6 & 20 & & & & 73 & 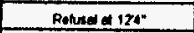 \\
\hline 3008 & 3014 & 12.2 & 30 August 1996 & $x$ & & 17 & 10 & $\frac{5}{6}$ & 4 & 3 & $\frac{7}{4}$ & 8 & $\frac{1}{4}$ & $\frac{1}{3}$ & $-\frac{2}{4}$ & $\frac{2}{14}$ & $\frac{2}{27}$ & 2 & & & 6 & 20 & & & & 99 & Petreated $1510 \%$ \\
\hline 3009 & 35 & 15.7 & 30 August 1996 & $x$ & & 20 & $\frac{\pi}{12}$ & $\frac{5}{5}$ & $\frac{7}{5}$ & $\frac{5}{5}$ & $\frac{7}{5}$ & 5 & 1 & $\frac{5}{1}$ & $\frac{4}{2}$ & $\frac{14}{1}$ & $\frac{4}{1}$ & 2 & 2 & 6 & $\bar{x}$ & & & & & 104 & Rensede a 1rT \\
\hline 3010 & 3016 & 15.9 & 30 August 1996 & $\bar{x}$ & & 17 & 10 & 6 & 6 & 5 & 4 & 4 & $\frac{1}{2}$ & $\frac{1}{1}$ & 9 & 0 & 0 & & & & 20 & & & & & 93 & Ponsed e 158 \\
\hline 3011 & 3017 & 14.1 & 30 AuQust 1996 & $\bar{x}$ & & 22 & 10 & 8 & 4 & 3 & 2 & 2 & & -1 & $\frac{1}{1}$ & $\frac{2}{3}+2+2+2$ & 0 & 4 & 8 & 17 & 25 & & & & & 110 & Beohese $154^{\circ}$ \\
\hline 3012 & 31 & 16.6 & 30 August 1996 & $\frac{a}{x}$ & & 20 & 10 & 3 & $\frac{9}{2}$ & $\frac{3}{2}$ & $\frac{6}{1}$ & $\frac{2}{4}$ & $\frac{1}{1}$ & $\frac{1}{1}$ & $\frac{1}{1}$ & 2 & 4 & 6 & 16 & 27 & & & & & & 109 & Retherere is \\
\hline 3013 & 3018 & 16.4 & 28 August 1996 & $\frac{\hat{x}}{x}$ & & 4 & $\sqrt{3}$ & $\frac{2}{2}$ & 2 & 1 & 1 & $\frac{1}{1}$ & $\frac{1}{1}$ & $\frac{1}{1}$ & $\frac{1}{4}$ & $\frac{4}{6}$ & $\frac{4}{30}$ & 6 & 6 & 5 & 20 & 10 & & & & 97 & Rolusenet ist \\
\hline 3014 & 3019 & 16.4 & 28 Auqust 1996 & $x$ & & 6 & 6 & 10 & 8 & 6 & $i$ & $\frac{1}{1}$ & $\frac{1}{2}$ & $\frac{1}{2}$ & $\frac{4}{4}$ & $\frac{6}{5}$ & $\frac{36}{5}$ & 42 & 26 & & & & & & & 130 & End of hout in $13 \%$ \\
\hline 3015 & 3020 & 150 & 28 August 1996 & $\frac{a}{x}$ & & 6 & 6 & 5 & 5 & $\frac{0}{3}$ & $\frac{1}{2}$ & $\frac{1}{2}$ & $\frac{2}{1}$ & $\frac{2}{1}$ & $\frac{4}{1}$ & $\frac{5}{3}$ & $\frac{5}{3}$ & 6 & 18 & 22 & 18 & 19 & & & & 139 & End of hole of 105 \\
\hline 3016 & $3 H$ & 13.1 & 30 August 1996 & $\frac{\hat{x}}{x}$ & & $\frac{8}{8}$ & $\frac{1}{6}$ & $\frac{2}{4}$ & $\frac{3}{4}$ & $\frac{\pi}{2}$ & $\frac{2}{2}$ & $\frac{2}{2}$ & $\frac{1}{2}$ & $\frac{1}{2}$ & $\frac{1}{4}$ & $\frac{8}{8}$ & $\frac{3}{9}$ & $\frac{3}{x}$ & 3 & 14 & & & & & & 58 & End of mon $\alpha 150$ \\
\hline 3017 & 3021 & 14.0 & 28 Auqust 1996 & $\frac{a}{x}$ & & 3 & $\frac{2}{2}$ & 2 & 1 & 1 & 1 & $\frac{2}{1}$ & $\frac{1}{1}$ & $\frac{2}{1}$ & 1 & $\frac{7}{1}$ & $\frac{5}{2}$ & $\frac{20}{18}$ & $\bar{M}$ & & & & & & & 73 & Polusene is 10 \\
\hline & & & & & & & & & & & & & & & & & & & & & & & & & & 119 & End of then a d 140 \\
\hline 3018 & 3022 & 20.0 & 28 August 1996 & $x$ & & 2 & 1 & 1 & 3 & 3 & 3 & 3 & 2 & 1 & 1 & 1 & 2 & 2 & 2 & 3 & 36 & 38 & 30 & & 3 & 143 & ind of then = 2000 Possibe \\
\hline 3010 & 3023 & 15.8 & 28 Auqust 1996 & $\frac{x}{x}$ & & 2 & 3 & 3 & 3 & 4 & 4 & 4 & 5 & 4 & 1 & 1 & 1 & 1 & $\frac{2}{1}$ & 1 & 25 & & & & & $\frac{143}{63}$ & Rocktmon mod Sa \\
\hline 3020 & 3024 & 15.5 & 28 August 1996 & $\bar{x}$ & & 4 & 4 & 5 & 4 & 3 & 3 & 1 & 1 & $\frac{1}{1}$ & 1 & 1 & 1 & 1 & 1 & $\frac{1}{1}$ & $\frac{26}{26}$ & & & & & 58 & Exc of thene 158 \\
\hline 3021 & 3025 & 13.7 & 28 Auqust 1996 & $x$ & & 4 & 5 & 3 & 3 & $\frac{5}{3}$ & 3 & 3 & 3 & 3 & 3 & 8 & 10 & 12 & 13 & & & & & & & 76 & Ens ol hot \& 138 \\
\hline 3022 & 3026 & 16.0 & 28 Auqust 1996 & $\bar{x}$ & & 4 & 5 & 1 & 1 & 1 & 1 & 1 & 1 & 1 & 1 & 1 & 1 & 2 & 5 & 6 & 78 & & & & & 50 & Ens a thes of $100^{\circ}$ \\
\hline 3023 & 3027 & 16.0 & 28 Auqust 1996 & $\frac{x}{x}$ & & 2 & 1 & 2 & 1 & 1 & 1 & 1 & 2 & 2 & 4 & 2 & 8 & 5 & 4 & $\frac{\pi}{1}$ & 30 & & & & & 67 & End ol how of 180 \\
\hline$\frac{3024}{3025}$ & $\frac{3028}{3030}$ & $\frac{16.1}{150}$ & 30 Auqust 1996 & $\frac{x}{x}$ & & 6 & 3 & 3 & 3 & 3 & 3 & 2 & 2 & 2 & 3 & 3 & 3 & 4 & 5 & 7 & 30 & & & & & 82 & Renre a I I $\mathrm{r}$ \\
\hline 3025 & 3029 & 15.0 & 28 Auqust 1996 & $\frac{x}{x}$ & & 4 & 4 & 4 & 5 & 7 & 5 & 4 & 4 & 5 & 5 & 6 & 8 & 8 & 12 & 12 & & & & & & 93 & End atroten is $15^{\circ} \sigma$ \\
\hline 3026 & 3030 & 16.0 & 28 AugL & $\frac{x}{x}$ & & 6 & 4 & 2 & 2 & 5 & 4 & 4 & 3 & 3 & 2 & 1 & 1 & 1 & $\frac{\pi}{1}$ & 1 & 22 & & & & & 62 & End of how of 1800 \\
\hline 3027 & 3031 & 16.0 & 30 August 1996 & $\underline{x}$ & & 4 & 3 & 2 & 2 & 2 & 2 & 2 & 2 & 1 & 2 & 1 & 1 & 2 & 2 & $\frac{2}{2}$ & 16 & & & & & 46 & Penses a a is \\
\hline 3028 & 3032 & 16.1 & 28 Auqust 1996 & $\bar{x}$ & & 3 & 4 & 4 & 5 & 4 & 4 & 4 & 3 & 2 & 3 & 3 & 2 & 2 & 2 & 2 & 26 & 36 & & & & 109 & End of hab a $161^{\circ}$ \\
\hline
\end{tabular}




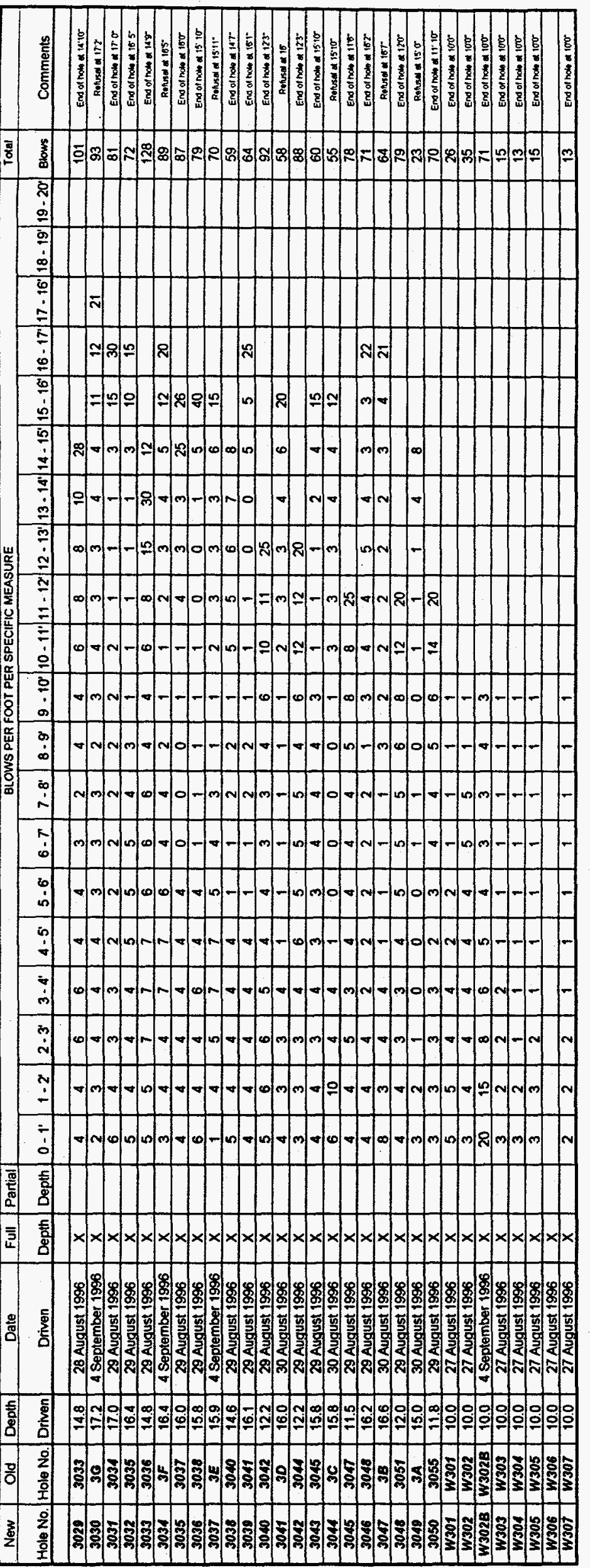




\begin{tabular}{|c|c|c|c|c|c|c|c|c|c|c|c|c|c|c|c|c|c|c|c|c|c|c|c|c|c|c|c|}
\hline New & Old & Depth & Date & Full & Partial & & & & & & & & BLOV & $S$ PER & $\infty D_{\text {PE }}$ & R SPEC & IFIC MEA & SURE & & & & & & & & Total & \\
\hline Hole No. & Hole No. & Driven & Driven & Depth & Depth & $0-1$ & 1.2 & $2-3^{\prime}$ & 3.4 & 4-5' & $5 \cdot 6$ & 6.7 & $7-8^{\prime}$ & $8-9^{\prime}$ & $9-10^{\circ}$ & $10-11$ & $11 \cdot 12$ & $12-13^{\prime}$ & $13 \cdot 14$ & $14-15$ & $15-16$ & $16-17$ & $17-16$ & $18-19$ & $19-20$ & Blows & Comments \\
\hline & & & & & & & & & & & & & & & & & & & & & & & & & & & \\
\hline 4001 & 1002 & 14.6 & 16 August 1996 & $x$ & & 6 & 6 & 6 & 8 & 8 & 9 & 9 & 12 & 12 & 12 & 12 & 12 & 10 & 10 & 10 & & & & & & 142 & Poss son rock Roused e ide? \\
\hline 1002 & .0003 & 14.4 & 16 August 1996 & $x$ & & 5 & 5 & 5 & 5 & 8 & 9 & 10 & 10 & 13 & 20 & 26 & 28 & 50 & 30 & 26 & & & & & & 250 & Poss son rock Reousese \\
\hline 4003 & 2004 & 13.3 & 16 Auqust 1996 & $\frac{x}{x}$ & & & & & & & & & & & & & & & & & & & & & & & 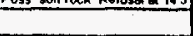 \\
\hline .004 & 4005 & 12.3 & 16 August 1996 & $x$ & & 6 & 6 & 5 & 4 & 3 & 3 & 3 & 3 & 2 & 5 & 6 & 12 & 13 & & & & & & & & 71 & Renssa at 1284: \\
\hline 4005 & 2006 & 12.2 & 16 August 1996 & $\bar{x}$ & & 5 & 4 & 4 & 3 & 3 & 2 & 2 & 2 & 6 & 8 & 6 & 8 & 18 & & & & & & & & 71 & Rehsse ef I I r \\
\hline 4006 & 4007 & 10.8 & 16 August 1996 & $x$ & & 4 & 4 & 4 & 4 & 5 & 5 & 5 & 4 & 4 & 18 & & & & & & & & & & & 57 & Ransose e 100 \\
\hline 1007 & 4008 & 11.3 & 16 August 1996 & $x$ & & 20 & 10 & 8 & 8 & 8 & 6 & 4 & 3 & 1 & 1 & 1 & 12 & & & & & & & & & 82 & 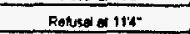 \\
\hline 4008 & 4000 & 9.6 & 16 August 1996 & $x$ & & 5 & 5 & 5 & 4 & 4 & 4 & 3 & 4 & 4 & 15 & & & & & & & & & & & 53 & Rethas a $97^{\circ}$ \\
\hline 4009 & .010 & 9.7 & 16 August 1996 & $\bar{x}$ & & 4 & 4 & 4 & 4 & 5 & 5 & 5 & 6 & 6 & 18 & & & & & & & & & & & 61 & Reursedere \\
\hline$\$ 010$ & 4011 & 11.5 & 16 August 1996 & $x$ & & 12 & 2 & 2 & 2 & 1 & 1 & 1 & 1 & 1 & 1 & 8 & 35 & & & & & & & & & 67 & Rensodellig \\
\hline 4011 & 4012 & 8.7 & 16 August 1996 & $\bar{x}$ & & 6 & 4 & 4 & 3 & 3 & 3 & 5 & 5 & 16 & & & & & & & & & & & & 49 & 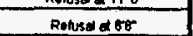 \\
\hline .012 & 4013 & 8.7 & 16 August 1996 & $x$ & & 5 & 4 & 4 & 4 & 4 & 4 & 4 & 6 & 18 & & & & & & & & & & & & 53 & Retuss a o $88^{\circ}$ \\
\hline 1013 & $\$ 014$ & 10.0 & 16 August 1996 & $x$ & & 6 & 4 & 4 & 4 & 2 & 2 & 2 & 20 & 48 & 90 & & & & & & & & & & & 182 & Retureate 100 \\
\hline 4014 & IA & 8.0 & 19 August 1996 & $x$ & & & & & & & & & & & & & & & & & & & & & & $\frac{106}{0}$ & 年 \\
\hline 4015 & 2016 & 9.3 & 16 August 1996 & $x$ & & 4 & 4 & 4 & 6 & 6 & 6 & 8 & 10 & 18 & 20 & & & & & & & & & & & 86 & 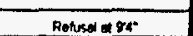 \\
\hline 1016 & 48 & 11.5 & 19 August 1996 & $\bar{x}$ & & & & & & & & & & & & & & & & & & & & & & & \\
\hline 4017 & 9017 & 9.7 & 16 August 1996 & $\bar{x}$ & & 4 & 2 & 2 & 4 & 4 & 8 & 8 & 12 & 9 & 19 & & & & & & & & & & & 72 & Reabsenterer \\
\hline 0098 & IC & 9.0 & 19 August 1996 & $x$ & & & & & & & & & & & & & & & & & & & & & & 12 & 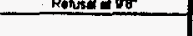 \\
\hline 4018 & $d E$ & 11.3 & 20 Auqust 1996 & $\bar{x}$ & & & & & & & & & & & & & & & & & & & & & & & \\
\hline 9020 & 4010 & 9.2 & 16 August 1996 & $x$ & & 4 & 4 & 5 & 6 & 6 & 6 & 8 & 8 & 12 & 20 & & & & & & & & & & & 79 & Rechessetery \\
\hline 4021 & 10 & 12.2 & 19 August 1996 & $x$ & & & & & & & & & & & & & & & & & & & & & & & \\
\hline 4022 & 9020 & 9.2 & 16 Auqust 1996 & $x$ & & 4 & 4 & 4 & 4 & 4 & 4 & 4 & 4 & 6 & 18 & & & & & & & & & & & 56 & 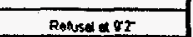 \\
\hline 4023 & 4047 & 12.8 & 19August 1996 & $x$ & & 20 & 15 & 10 & 6 & 6 & 6 & 6 & 10 & 6 & 6 & 6 & 7 & 17 & & & & & & & & 121 & Rearsed a 128 \\
\hline 2024 & 1022 & 9.2 & 16 August 1996 & $x$ & & 4 & 4 & 4 & 4 & 5 & 5 & 6 & 8 & 8 & 12 & & & & & & & & & & & 60 & Rensed of $\theta$ r \\
\hline 4025 & 1023 & 9.1 & 16 August 1996 & $x$ & & 5 & 5 & 6 & 6 & 6 & 6 & 8 & 8 & 6 & 16 & & & & & & & & & & & $\frac{00}{72}$ & Rothese a git \\
\hline 4026 & 1048 & 12.3 & 19 August 1996 & $x$ & & 6 & 4 & 4 & 4 & 2 & 2 & 2 & 2 & 3 & 2 & 4 & 7 & 17 & & & & & & & & 59 & 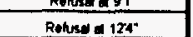 \\
\hline 2027 & $\$ 024$ & 9.7 & 16 August 1996 & $x$ & & 4 & 4 & 4 & 6 & 6 & 8 & 12 & 12 & 15 & 16 & & & & & & & & & & & 87 & Rensed a d of \\
\hline 9028 & 2049 & 12.3 & 19 August 1996 & $\bar{x}$ & & 20 & 18 & 6 & 5 & 4 & 3 & 4 & 4 & 4 & 4 & 4 & 6 & 16 & & & & & & & & $\frac{01}{98}$ & 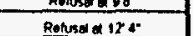 \\
\hline
\end{tabular}


PIPE DRIVING SUMMARY

\begin{tabular}{|c|c|c|c|c|c|c|c|c|c|c|c|c|c|c|c|c|c|c|c|c|c|c|c|c|c|c|c|}
\hline New & old & Depth & Date & Full & Parrial & & & & & & & & & $S$ SPER & FOT PI & R SPEC & FIC ME & ASURE & & & & & & & & Total & \\
\hline Hole No. & tole No. & Driven & Driven & Depth & Depth & $0.1^{\prime}>->$ & $1-2$ & $2 \cdot 3^{3}$ & $3-4^{\prime}$ & 4. $5^{\prime}$ & $5-6$ & $6 \cdot 7$ & $7 \cdot 8^{\prime}$ & 8.9 & & -1 & $1-$ & $12-13$ & {$[13.14$} & {$[14-15$} & $415 \cdot 16$ & $|16 \cdot 177|$ & $17-16^{\prime}$ & $\mid 18 \cdot 19$ & $19-20$ & Blows & Comments \\
\hline 6029 & If & 9.3 & 20 August 1996 & $\bar{x}$ & & & & & & & & & & & & & & & & & & & & & & & \\
\hline 1030 & 1026 & 8.5 & 16 August 1996 & $\bar{x}$ & & 4 & 4 & 4 & 5 & 5 & 5 & 6 & 7 & 17 & & & & & & & & & & & & 57 & Reabsued ar \\
\hline$\$ 031$ & 1050 & 12.4 & 19 August 1996 & $x$ & & 20 & 20 & 8 & 8 & 3 & 3 & 3 & 3 & 3 & 3 & 3 & 5 & 15 & & & & & & & & 97 & Rethso ex 125 \\
\hline$\frac{2032}{4032}$ & 4027 & 9.8 & 16 August 1996 & $\frac{x}{x}$ & & 4 & 4 & 4 & 4 & 3 & 10 & 12 & 12 & 14 & & & & & & & & & & & & 67 & Pensed a o f i 10 \\
\hline 4033 & 2051 & $\frac{12.5}{98}$ & 19 August 1996 & $\frac{x}{x}$ & & $\frac{12}{1}$ & 12 & 10 & $\frac{6}{4}$ & 4 & 4 & $-\frac{4}{12}$ & 4 & 6 & 6 & 6 & 16 & & & & & & & & & 90 & Renssed a $1280^{\circ}$ \\
\hline$\frac{4034}{4025}$ & \begin{tabular}{|c|c|}
.028 \\
053
\end{tabular} & $\frac{8.6}{128}$ & 16 August 1996 & $\frac{x}{x}$ & & $\frac{4}{30}$ & $\frac{4}{12}$ & $\frac{4}{8}$ & \begin{tabular}{|l}
4 \\
5
\end{tabular} & $\frac{8}{5}$ & 12 & $\frac{12}{1}$ & $\frac{12}{4}$ & $\frac{25}{3}$ & & & & & & & & & & & & $\begin{array}{ll}85 \\
104 \\
104\end{array}$ & Retusat e o or \\
\hline$\frac{4035}{4036}$ & \begin{tabular}{|l}
4052 \\
4030
\end{tabular} & $\frac{12.8}{97}$ & $\frac{19 \text { August } 1996}{19 \text { Auqust }}$ & $\frac{x}{x}$ & - & $\frac{30}{5}$ & $\frac{12}{5}$ & $\frac{8}{5}$ & \begin{tabular}{|l|}
5 \\
5
\end{tabular} & $\frac{5}{5}$ & \begin{tabular}{|l|}
8 \\
8
\end{tabular} & $\frac{4}{12}$ & $\frac{4}{15}$ & $\frac{3}{20}$ & $\frac{3}{40}$ & 5 & 5 & 16 & & & & & & & & $\frac{104}{120}$ & 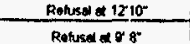 \\
\hline 4037 & 4053 & 12.8 & 19 August 1996 & $x$ & & 12 & 8 & 6 & 3 & 3 & 3 & 4 & 3 & $\frac{2}{3}$ & 2 & 2 & 2 & 15 & & & & & & & & 66 & Rotusseder $1710^{\circ}$ \\
\hline 4038 & 40 & 8.9 & 20 August 1996 & $x$ & & & & & & & & & & & & & & & & & & & & & & & \\
\hline 2039 & 4032 & $\frac{97}{125}$ & 19 August 1996 & $\frac{x}{y}$ & & 5 & 5 & 5 & 5 & 6 & $\frac{6}{1}$ & 12 & 12 & 18 & 25 & & & & & & & & & & & 99 & Reterace of \\
\hline 4040 & 4033 & $\frac{13.5}{13}$ & 19August 1996 & $\frac{x}{x}$ & & $\frac{12}{12}$ & $\frac{12}{8}$ & 8 & 4 & 4 & 4 & 4 & 4 & 4 & 4 & 4 & $\frac{10}{2}$ & 12 & 15 & & & & & & & 101 & Pensede 13\% \\
\hline$\frac{4041}{4042}$ & $\frac{4054}{4055}$ & $\frac{13}{12.9}$ & $\frac{19 \text { August } 1996}{\text { 19August 1996 }}$ & $\frac{x}{x}$ & - & $\frac{12}{6}$ & $\frac{8}{6}$ & $\frac{5}{4}$ & \begin{tabular}{|l|}
3 \\
4
\end{tabular} & $\frac{3}{4}$ & $-\frac{3}{4}$ & $\frac{3}{4}$ & $\frac{3}{4}$ & $\frac{3}{4}$ & $\frac{2}{5}$ & $\frac{2}{5}$ & $\frac{2}{8}$ & $\frac{15}{10}$ & & & - & 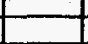 & & & - & \begin{tabular}{|l|}
64 \\
68 \\
\end{tabular} & 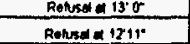 \\
\hline$\frac{2043}{4043}$ & 8034 & 9.7 & 19August 1996 & $\hat{x}$ & & 4 & 4 & 4 & 5 & 6 & 8 & 12 & 12 & $\frac{15}{15}$ & 20 & & & & & & & & & & & \begin{tabular}{|c|}
90 \\
\end{tabular} & Reveredear \\
\hline 1044 & 4036 & 13 & 19 August 1996 & $x$ & & 5 & 5 & 5 & 5 & 5 & 5 & 5 & 5 & 4 & 3 & 4 & 6 & 15 & & & & & & & & 72 & Rensoua a 130 \\
\hline 4045 & 4056 & 10.0 & 19 Auqust 1996 & & & & & & & & & & & & & & & & & & & & & & & & \\
\hline 4046 & 4037 & $\begin{array}{l}8.2 \\
100\end{array}$ & 19 Auqust 1996 & $\frac{x}{y}$ & & 4 & 3 & 3 & 4 & 5 & 10 & 12 & 12 & 29 & & & & & & & & & & & & 82 & \\
\hline 4048 & $\frac{6057}{4 H}$ & $\frac{12.8}{150}$ & $\frac{19 \text { August } 1996}{20 \text { Aunoust } 1999}$ & $\frac{x}{x}$ & & & & & & & & & & & & & & & & & & & & & & & \\
\hline 4049 & 4039 & \begin{tabular}{|c|}
13.3 \\
\end{tabular} & 19 August 1996 & $x$ & & 4 & 4 & 5 & 6 & 6 & 6 & 4 & 3 & 3 & 3 & 2 & 12 & 12 & 26 & & & & & & & 96 & 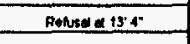 \\
\hline$\frac{1050}{1050}$ & 4058 & 8.7 & 19 August 1996 & $\frac{x}{x}$ & & & & & & & & & & & & & & & & & & & & & & & \\
\hline 2051 & 2040 & \begin{tabular}{|l|}
9.8 \\
12
\end{tabular} & $19 \mathrm{Aug}$ & $\frac{x}{x}$ & & 6 & 8 & 10 & 12 & 12 & 10 & 10 & 12 & 14 & 16 & & & & & & & & & & & $\frac{110}{8}$ & Rotres a \& p p10 \\
\hline 4052 & 4059 & 14.2 & 19 Aus & $\frac{x}{y}$ & & & & & & & & & & & & & & & & & & & & & & 0 & \\
\hline 4053 & 4042 & $\frac{13.2}{1.0}$ & $19 \mathrm{Au}$ & $\frac{x}{x}$ & & $\frac{12}{30}$ & 6 & 4 & 5 & 4 & 4 & 3 & -3 & 3 & 6 & $-\frac{8}{9}$ & $\frac{8}{10}$ & $+\frac{10}{x}$ & 15 & & & & & & & 91 & Rensed of i $13{ }^{\prime} r$ \\
\hline$\frac{4054}{4055}$ & \begin{tabular}{|l}
4043 \\
4048
\end{tabular} & $\frac{12.9}{127}$ & $\frac{1 \text { AAAus }}{\text { 19Aug }}$ & $\frac{x}{x}$ & - & $\frac{30}{60}$ & $\frac{18}{30}$ & $\frac{6}{15}$ & $\frac{6}{8}$ & $\frac{4}{6}$ & -4 & $\frac{4}{4}$ & $\frac{4}{3}$ & $\frac{4}{4}$ & $\frac{8}{3}$ & $\frac{8}{3}$ & $\frac{10}{3}$ & $\frac{20}{14}$ & & & & & 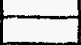 & & & $\mid$\begin{tabular}{|l|l|}
157 \\
157
\end{tabular} & 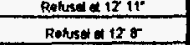 \\
\hline 4056 & 4046 & 12.9 & 16 Auqust 1996 & $x$ & & 5 & 5 & 5 & 4 & 4 & 3 & 3 & 4 & $\frac{7}{2}$ & 1 & 8 & 8 & 26 & & & & & & & & 78 & Resure o a 12 it \\
\hline W401 & $\begin{array}{l}\text { W401 } \\
\text { werp? }\end{array}$ & $\frac{10.0}{10 n}$ & 16 Auqust 1996 & $\frac{x}{x}$ & & & & & & & & & & & & & & & & & & & & & & & \\
\hline W402 & W402 & 10.0 & 16 August 1996 & $x$ & & & & & & & & & & & & & & & & & & & & & & & \\
\hline W403 & w403 & 10.0 & $16 \mathrm{Au}$ & $\underline{\lambda}$ & & 5 & 5 & 3 & 5 & 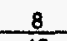 & 3 & 10 & 10 & 13 & 20 & 26 & 28 & 50 & 30 & 26 & & & & & & 250 & son rocke \\
\hline (1) & W404 & no & & $\bar{x}$ & & 18 & 20 & 22 & 20 & 15 & 2 & 2 & 2 & 10 & 8 & 8 & 8 & 8 & 16 & & & & & & & 159 & Enat a 13:- \\
\hline W40 & WA05 & 8.0 & $16 \mathrm{~A}$ & $\bar{x}$ & & & & & & & & & & & & & & & & & & & & & & & \\
\hline$w 40$ & W406 & 10.0 & & & & & & & & & & & & & & & & & & & & & & & & & \\
\hline Wat & W403B & $\frac{10.0}{100}$ & $\frac{27 A}{27 A}$ & $\frac{x}{y}$ & & & & & & & & & & & & & & & & & & & & & & & \\
\hline$W 4048$ & $\frac{W 408}{W 405 B}$ & $\frac{10.0}{100}$ & $\begin{array}{l}27 \text { Auqust } 1996 \\
27 \text { Auqust } 1996\end{array}$ & $x$ & & & & & & & & & & & & & & & & & & & & & & & \\
\hline
\end{tabular}




\section{APPENDIX B}

CASING GROUT INJECTION RECORDS 


\section{ACT REMBCO JOINT VENTURE \\ Oml Wag 4 Seeps 4 \& 6 Removal Action Project \\ Casing Grout Table}

Date: July 29, 1996

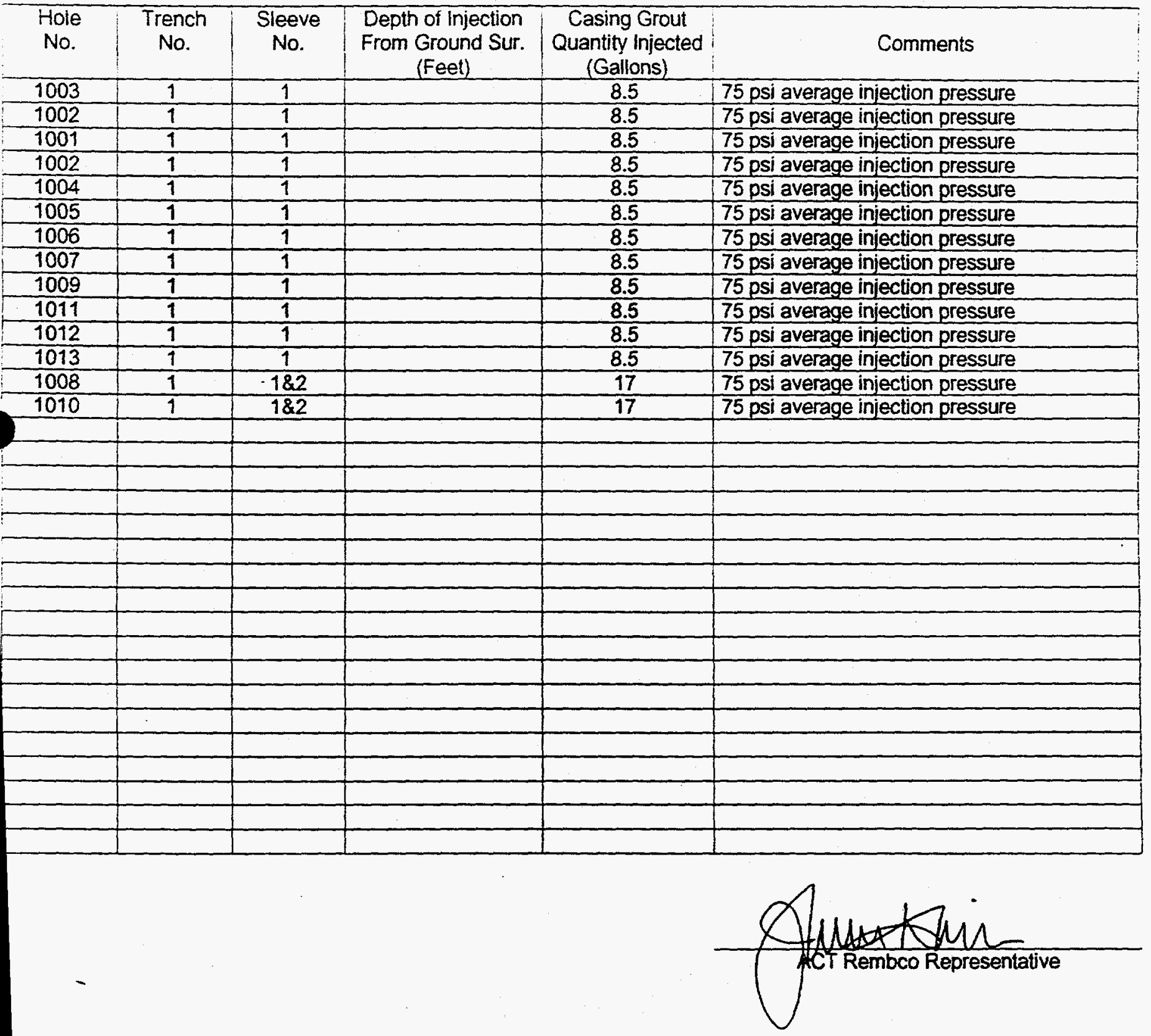




\title{
ACT REMBCO JOINT VENTURE \\ Ornl Wag 4 Seeps 4 \& 6 Removal Action Project
}

\author{
Casing Grout Table
}

Date: July 30, 1996

\begin{tabular}{|c|c|c|c|c|c|}
\hline $\begin{array}{l}\text { Hole } \\
\text { No. }\end{array}$ & $\begin{array}{c}\text { Trench } \\
\text { No. }\end{array}$ & $\begin{array}{c}\text { Sleeve } \\
\text { No. }\end{array}$ & $\begin{array}{l}\text { Depth of Injection } \\
\text { From Ground Sur. } \\
\text { (Feet) }\end{array}$ & $\begin{array}{c}\text { Casing Grout } \\
\text { Quantity Injected } \\
\text { (Gallons) }\end{array}$ & Comments \\
\hline 1001 & 1 & 2 & & 8.5 & 65 psi average injection pressure \\
\hline 1001 & 1 & 3 & & 8.5 & 65 psi average injection pressure \\
\hline 1001 & 1 & 4 & & 8.5 & 65 psi average injection pressure \\
\hline 1001 & 1 & 5 & & 8.5 & 65 psi average injection pressure \\
\hline 1001 & $T$ & 6 & & 8.5 & 65 psi average injection pressure \\
\hline 1001 & 1 & 7 & & 8.5 & 65 psi average injection pressure \\
\hline 1002 & 1 & 2 & & 8.5 & 65 psi average injection pressure \\
\hline 1002 & 1 & 3 & & 8.5 & 65 psi average injection pressure \\
\hline 1002 & 1 & 4 & 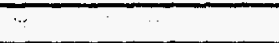 & 8.5 & 65 psi average injection pressure \\
\hline 1002 & 1 & 5 & & 8.5 & 65 psi average injection pressure \\
\hline 1002 & 1 & 6 & & 8.5 & 65 psi average injection pressure \\
\hline 1002 & $T$ & 7 & & 8.5 & $65 \mathrm{psi}$ average injection pressure \\
\hline 1003 & 1 & 2 & & 8.5 & 65 psi average injection pressure \\
\hline 1003 & 1 & 3 & & 8.5 & 65 psi average injection pressure \\
\hline 1003 & 1 & 4 & & 8.5 & 65 psi average injection pressure \\
\hline 1003 & 1 & 5 & & 8.5 & 65 psi average injection pressure \\
\hline 1003 & 1 & 6 & & 8.5 & 65 psi average injection pressure \\
\hline 1003 & 1 & 7 & & 8.5 & 65 psi average injection pressure \\
\hline 1003 & 1 & 8 & & 8.5 & 65 psi average injection pressure \\
\hline 1004 & $T$ & 2 & & 8.5 & 65 psi average injection pressure \\
\hline 1004 & 1 & 3 & & 8.5 & 65 psi average injection pressure \\
\hline 1004 & 1 & 4 & & 8.5 & 65 psi average injection pressure \\
\hline & & & & & \\
\hline & & & & & \\
\hline & & & & & \\
\hline & & & & & \\
\hline & & & & & \\
\hline & & & & & \\
\hline & & & & & \\
\hline & & & & & \\
\hline & & & & & \\
\hline & & & & & \\
\hline & & & & & \\
\hline & & & & & \\
\hline & & & & & \\
\hline
\end{tabular}

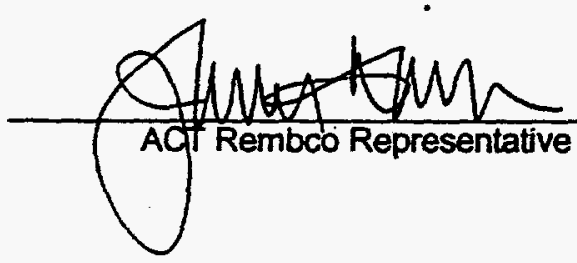




\section{ACT REMBCO JOINT VENTURE \\ Ornl Wag 4 Seeps 4 \& 6 Removal Action Project}

Casing Grout Table

Date: August 02, 1996

\begin{tabular}{|c|c|c|c|c|c|}
\hline $\begin{array}{l}\text { Hole } \\
\text { No. }\end{array}$ & $\begin{array}{c}\text { Trench } \\
\text { No. }\end{array}$ & $\begin{array}{c}\text { Sleeve } \\
\text { No. }\end{array}$ & $\begin{array}{l}\text { Depth of injection } \\
\text { From Ground Sur. } \\
\text { (Feet) }\end{array}$ & $\begin{array}{c}\text { Casing Grout } \\
\text { Quantity Injected } \\
\text { (Litres) }\end{array}$ & Comments \\
\hline 1004 & 1 & $5=$ & 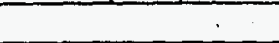 & 25 & 30 psi average injection pressure \\
\hline 1004 & 1 & $6=$ & & 25 & 30 psi average iniection pressure \\
\hline 1005 & 1 & 2 & & 25 & 30 psi average injection pressure \\
\hline 1004 & 1 & 7 - & & 25 & 30 psi average injection pressure \\
\hline 1005 & 1 & $3^{2}$ & & 25 . & 30 psi average injection pressure \\
\hline 1007 & 1 & 2 & & 25 & 30 psi average injection pressure \\
\hline 1007 & 1 & 3 & & 25 & 30 psi average injection pressure \\
\hline 1007 & 1 & 4 & & 25 & 30 psi average injection pressure \\
\hline 1007 & 1 & $5-$ & 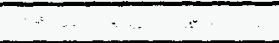 & 25 & 30 psi average injection pressure \\
\hline 1007 & 1 & $6-$ & & 25 & 30 psi average injection pressure \\
\hline 1007 & 1 & $7=$ & & 25 & 30 psi average injection pressure \\
\hline 1005 & 1 & 4 & & 25 & 30 psi average injection pressure \\
\hline 1005 & 1 & 5 & & 25 & 30 psi average injection pressure \\
\hline 1006 & 1 & $4-$ & & 50 & 30 psi average injection pressure \\
\hline 1006 & $\overline{1}$ & $5-$ & & 25 & 30 psi average injection pressure \\
\hline 1006 & 1 & $6^{-}$ & & 25 & 30 psi average injection pressure \\
\hline 1006 & 1 & 7 & & 25 & 30 psi average injection pressure \\
\hline 1008 & 1 & $3 !$ & & 25 & 30 psi average injection pressure \\
\hline 1008 & 1 & 4 & & 25 & 30 psi average injection pressure \\
\hline 1008 & 1 & 5 & & 25 & 30 psi average injection pressure \\
\hline 1008 & 1 & 6 & & 25 & 30 psi average injection pressure \\
\hline 1008 & 1 & 7 & & 25 & 30 psi average injection pressure \\
\hline 1009 & 1 & 2, & & 25 & 30 psi average injection pressure \\
\hline 1009 & 1 & 3 & & 25 & 30 psi average injection pressure \\
\hline 1009 & 1 & $\overline{4}$ & & 25 & 30 psi average injection pressure \\
\hline 1009 & 1 & 5 & & 25 & 30 psi average injection pressure \\
\hline 1009 & 1 & 67 & & 25 & 30 psi average injection pressure \\
\hline 1010 & 1 & $\frac{5}{3}$ & & 25 & 30 psi average injection pressure \\
\hline 1010 & $\overline{1}$ & 4 & & 25 & 30 psi average injection pressure \\
\hline 1010 & $\overline{1}$ & 5 & & 25 & 30 psi average injection pressure \\
\hline 1010 & $\overline{1}$ & 6 & & 25 & 30 psi average injection pressure \\
\hline 1010 & 1 & 7 & & 25 & 30 psi average injection pressure \\
\hline 1011 & 1 & 2 & & 25 & 30 psi average injection pressure \\
\hline 1011 & 1 & 3 & & 25 & 30 psi average injection pressure \\
\hline 1011 & 1 & 4 & & 25 & 30 psi average injection pressure \\
\hline 1011 & 1 & 5 & & 25 & 30 psi average injection pressure \\
\hline 1014 & 1 & 6 & & 25 & 30 psi average injection pressure \\
\hline 1011 & 1 & 7 & & 25 & 30 psi average injection pressure \\
\hline 1011 & 1 & 81 & & 25 & 30 psi average injection pressure \\
\hline 1012 & 1 & 2 & & 25 & 30 psi average injection pressure \\
\hline 1012 & 1 & $3 !$ & & 25 & 30 psi average injection pressure \\
\hline$\longdiv { 1 0 1 2 }$ & $\overline{1}$ & $\overline{4}$ & & 25 & 30 psi average injection pressure \\
\hline
\end{tabular}




\section{ACT REMBCO JOINT VENTURE}

Oml Wag 4 Seeps 4 \& 6 Removal Action Project

Casing Grout Table

Date: August 02, 1996

\begin{tabular}{|c|c|c|c|c|c|}
\hline $\begin{array}{l}\text { Hole } \\
\text { No. }\end{array}$ & $\begin{array}{c}\text { Trench } \\
\text { No. }\end{array}$ & $\begin{array}{c}\text { Sleeve } \\
\text { No. }\end{array}$ & $\begin{array}{l}\text { Depth of Injection } \\
\text { From Ground Sur. } \\
\text { (Feet) }\end{array}$ & $\begin{array}{c}\text { Casing Grout } \\
\text { Quantity Injected } \\
\text { (Litres) }\end{array}$ & Comments \\
\hline 1012 & 1 & 5 & & 25 & 30 psi average injection pressure \\
\hline 1012 & 1 & 6 & & 25 & 30 psi average injection pressure \\
\hline 1012 & 1 & 7 & & 25 & 30 psi average injection pressure \\
\hline 1013 & 1 & 2 & & 25 & 30 psi average injection pressure \\
\hline 1013 & 1 & $3 !$ & & 25 & 30 psi average injection pressure \\
\hline 1013 & 1 & $\overline{4}$ & & 25 & 30 psi average injection pressure \\
\hline 1013 & 1 & 5 & & 25 & $30 \mathrm{psi}$ average injection pressure \\
\hline 1013 & $T$ & $\overline{6}$ & & 25 & 30 psi average injection pressure \\
\hline 1013 & 1 & $\overline{7}$ & & 25 & 30 psi average injection pressure \\
\hline 1013 & 1 & 8 & & 25 & 30 psi average injection pressure \\
\hline 1014 & 1 & $1=$ & & 25 & 60 psi average injection pressure \\
\hline 1015 & 1 & $1,2,3$ & & 75 & 60 psi average injection pressure \\
\hline 1016 & 1 & $1-$ & & 25 & 60 psi average injection pressure \\
\hline 1017 & 1 & 1 & & 25 & 60 psi average injection pressure \\
\hline 1018 & 1 & 9 & & 25 & 60 psi average injection pressure \\
\hline 1021 & 1 & 1- & & 25 & 60 psi average injection pressure \\
\hline 1022 & 1 & 1 & & 25 & 60 psi average injection pressure \\
\hline 1023 & 1 & $182-$ & & 50 & 60 psi average injection pressure \\
\hline 1024 & 1 & $1-$ & & 25 & 60 psi average injection pressure \\
\hline 1026 & 1 & $1-$ & & 25 & 60 psi average injection pressure \\
\hline 1027 & 1 & $1 \& 2-$ & & 50 & 60 psi average injection pressure \\
\hline 1028 & 1 & $182 /$ & & 50 & 60 psi average injection pressure \\
\hline 1029 & 9 & $1-$ & & 25 & 60 psi average injection pressure \\
\hline 1032 & 1 & $182-$ & & 50 & 60 psi average injection pressure \\
\hline 1033 & 1 & $1-$ & & 25 & $60 \mathrm{psi}$ average injection pressure \\
\hline 1035 & 1 & 15 & & 25 & 60 psi average injection pressure \\
\hline 1036 & 1 & 1 & & 25 & 60 psi average injection pressure \\
\hline 1037 & 1 & $1 \div$ & & 25 & 60 psi average injection pressure \\
\hline 1019 & 1 & $1=$ & & 25 & 60 psi average injection pressure \\
\hline 1020 & 1 & $1=$ & & 25 & 60 psi average injection pressure \\
\hline 1025 & 1 & $182=$ & & 50 & 60 psi average injection pressure \\
\hline 1030 & 1 & $1=$ & & 25 & 60 psi average injection pressure \\
\hline 1031 & 1 & $1=$ & & 25 & 60 psi average injection pressure \\
\hline 1014 & 1 & 2 & & 25 & 60 psi average injection pressure \\
\hline 1014 & 1 & 3 & & 25 & 60 psi average injection pressure \\
\hline 1014 & 1 & 4 & & 25 & 60 psi average injection pressure \\
\hline 1014 & 1 & 51 & & 25 & 60 psi average injection pressure \\
\hline 1014 & 1 & 6 & & 25 & 60 psi average injection pressure \\
\hline 1014 & $\overline{1}$ & 7 & & 25 & 60 psi average injection pressure \\
\hline 1014 & 1 & 8 & & 25 & 60 psi average injection pressure \\
\hline 1015 & 1 & 41 & & 25 & 60 psi average injection pressure \\
\hline 1015 & 1 & 57 & & 25 & 60 psi average injection pressure \\
\hline
\end{tabular}




\title{
ACT REMBCO JOINT VENTURE \\ Ornl Wag 4 Seeps 4 \& 6 Removal Action Project
}

\author{
Casing Grout Table
}

Date: August 02, 1996

\begin{tabular}{|c|c|c|c|c|c|}
\hline $\begin{array}{l}\text { Hole } \\
\text { No. }\end{array}$ & $\begin{array}{c}\text { Trench } \\
\text { No. }\end{array}$ & $\begin{array}{c}\text { Sleeve } \\
\text { No. }\end{array}$ & $\begin{array}{c}\text { Depth of Injection } \\
\text { From Ground Sur. } \\
\text { (Feet) }\end{array}$ & $\begin{array}{c}\text { Casing Grout } \\
\text { Quantity Injected } \\
\text { (Litres) }\end{array}$ & Comments \\
\hline 1015 & 1 & $\overline{6}$ & & 25 & 60 psi average injection pressure \\
\hline 1015 & 1 & 7 & & 25 & 60 psi average injection pressure \\
\hline 1015 & 1 & 8 & & 25 & 60 psi average injection pressure \\
\hline 1016 & $T$ & 2 & & 25 & 60 psi average injection pressure \\
\hline 1016 & 1 & 3 & & 25 & 60 psi average injection pressure \\
\hline 1016 & 1 & $4:$ & & 25 & 60 psi average injection pressure \\
\hline 1016 & 1 & 5 & & 25 & 60 psi average injection pressure \\
\hline 1016 & 1 & 6 & & 25 & 60 psi average injection pressure \\
\hline 1016 & 1 & 7 & & 25 & 60 psi average injection pressure \\
\hline 1016 & 1 & 8 & & 25 & 60 psi average injection pressure \\
\hline 1017 & 1 & 2 & & 25 & 60 psi average injection pressure \\
\hline 1017 & 1 & 3 & & 25 & 60 psi average injection pressure \\
\hline 1017 & 1 & $\overline{4}$ & & 25 & 60 psi average injection pressure \\
\hline 1017 & 1 & $\overline{5}$ & & 25 & 60 psi average injection pressure \\
\hline 1017 & 1 & 6 & & 25 & 60 psi average injection pressure \\
\hline 1017 & $\frac{T}{1}$ & 7 & & 25 & 60 psi average injection pressure \\
\hline 1017 & 1 & 8 & & 25 & 60 psi average injection pressure \\
\hline 1018 & 1 & $2 !$ & & 25 & 60 psi average injection pressure \\
\hline 1018 & 1 & 3 & & 25 & 60 psi average injection pressure \\
\hline 1018 & 1 & 4 & & 25 & 60 psi average injection pressure \\
\hline 1018 & 1 & 5 & & 25 & 60 psi average injection pressure \\
\hline 1018 & 1 & 6 & & 25 & 60 osi average injection pressure \\
\hline 1018 & 1 & 7 & & 25 & 60 psi average injection pressure \\
\hline 1021 & 1 & 2 & & 25 & 60 psi average injection pressure \\
\hline 1021 & 1 & $\overline{3}$ & & 25 & 60 psi average injection pressure \\
\hline 1021 & 1 & 4 & & 25 & 60 psi average injection pressure \\
\hline 1021 & 1 & $\overline{5}$ & & 25 & 60 psi average injection pressure \\
\hline 1021 & 1 & 6 & & 25 & 60 psi average injection pressure \\
\hline 1021 & 1 & 71 & & 25 & 60 psi average injection pressure \\
\hline 1022 & 1 & 21 & & 25 & 60 psi average injection pressure \\
\hline 1022 & 1 & $\overline{3}$ & & 25 & 60 psi average injection pressure \\
\hline 1022 & $T$ & 4 & & 25 & 60 psi average injection pressure \\
\hline 1022 & 1 & 5 & & 25 & 60 psi average injection pressure \\
\hline 1022 & 1 & 6 & & 25 & 60 psi average injection pressure \\
\hline 1022 & 1 & 7 & & 25 & 60 psi average injection pressure \\
\hline 1023 & $T$ & $3 \& 4$ & & 50 & 60 psi average injection pressure \\
\hline 1023 & 1 & $5 \%$ & & 25 & 60 psi average injection pressure \\
\hline 1023 & 1 & 6 & & 25 & 60 psi average injection pressure \\
\hline 1023 & 1 & 7 & & 25 & 60 psi average injection pressure \\
\hline 1024 & 1 & $2 !$ & & 25 & 60 psi average injection pressure \\
\hline 1024 & 1 & 3 & & 25 & 60 psi average injection pressure \\
\hline 1024 & 1 & 4 & & 25 & 60 psi average injection pressure \\
\hline
\end{tabular}


ACT REMBCO JOINT VENTURE

Ornl Wag 4 Seeps 4 \& 6 Removal Action Project

Casing Grout Table

Date: August 02, 1996

\begin{tabular}{|c|c|c|c|c|c|}
\hline $\begin{array}{c}\text { Hole } \\
\text { No. }\end{array}$ & $\begin{array}{c}\text { Trench } \\
\text { No. }\end{array}$ & $\begin{array}{c}\text { Sleeve } \\
\text { No. }\end{array}$ & $\begin{array}{c}\text { Depth of Injection } \\
\text { From Ground Sur. } \\
\text { (Feet) }\end{array}$ & $\begin{array}{c}\text { Casing Grout } \\
\text { Quantity Injected } \\
\text { (Litres) }\end{array}$ & Comments \\
\hline 1024 & 1 & 5 & & 25 & 60 psi average injection pressure \\
\hline 1024 & 1 & 6 & & 25 & 60 psi average injection pressure \\
\hline 1024 & 1 & 7 & & 25 & 60 psi average injection pressure \\
\hline 1026 & 1 & 2 & & 25 & 60 psi average injection pressure \\
\hline 1026 & 1 & 3 & & 25 & 60 psi average injection pressure \\
\hline 1026 & 1 & 4 & & 25 & 60 psi average injection pressure \\
\hline 1026 & 1 & 5 & & 25 & 60 psi average injection pressure \\
\hline 1026 & 1 & 6 & & 25 & 60 psi average injection pressure \\
\hline 1026 & 1 & 7 & & 25 & 60 psi average injection pressure \\
\hline 1026 & 1 & 8 & & 25 & 60 psi average injection pressure \\
\hline
\end{tabular}

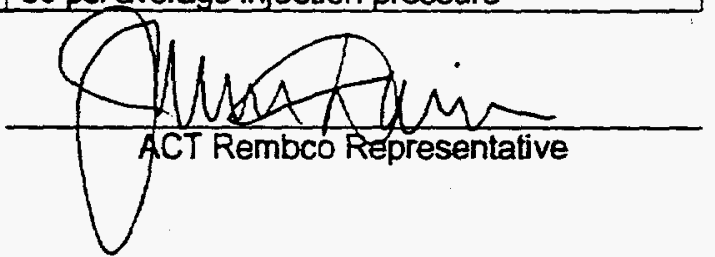




\title{
ACT REMBCO JOINT VENTURE \\ Oml Wag 4 Seeps 4 \& 6 Removal Action Project
}

\author{
Casing Grout Table
}

Date: August 06, 1996

\begin{tabular}{|c|c|c|c|c|c|}
\hline $\begin{array}{l}\text { Hole } \\
\text { No. }\end{array}$ & $\begin{array}{c}\text { Trench } \\
\text { No. }\end{array}$ & $\begin{array}{c}\text { Sleeve } \\
\text { No. }\end{array}$ & $\begin{array}{l}\text { Depth of injection } \\
\text { From Ground Sur. } \\
\text { (Feet) }\end{array}$ & $\begin{array}{c}\text { Casing Grout } \\
\text { Quantity injected } \\
\text { (Litres) }\end{array}$ & Comments \\
\hline 1019 & $\overline{1}$ & 2 & & 25 & 65 psi average injection pressure \\
\hline 1020 & 1 & $\overline{2}$ & & 25 & 65 psi average injection pressure \\
\hline 1019 & 1 & 3 & & 25 & 65 psi average injection pressure \\
\hline 1020 & 1 & 3 & & 25 & 65 psi average injection pressure \\
\hline 1019 & 1 & $\overline{4}$ & & 25 & 65 psi average injection pressure \\
\hline 1020 & 1 & 4 & & 25 & 65 psi average injection pressure \\
\hline 1019 & 1 & 5 & & 25 & 65 psi average injection pressure \\
\hline 1020 & 1 & 5 & & 25 & 65 psi average injection pressure \\
\hline 1019 & 1 & 6 & & 25 & 65 psi average injection pressure \\
\hline 1020 & 1 & $\overline{6}$ & & 25 & 65 psi average injection pressure \\
\hline 1019 & 1 & 7 & & 25 & 65 psi average injection pressure \\
\hline 1025 & 1 & 3 & & 25 & 65 psi average injection pressure \\
\hline 1027 & 1 & $\overline{3}$ & & 25 & 65 psi average injection pressure \\
\hline 1025 & 1 & 4 & & 25 & 65 psi average injection pressure \\
\hline 1027 & 1 & 4 & & 25 & 65 psi average injection pressure \\
\hline 1025 & 1 & 5 & & 25 & 65 psi average injection pressure \\
\hline 1027 & 1 & 5 & & 25 & 65 psi average injection pressure \\
\hline 1025 & 1 & 6 & & 25 & 65 psi average injection pressure \\
\hline 1027 & 1 & 6 & & 25 & 65 psi average injection pressure \\
\hline 1028 & 1 & 3 & & 25 & 65 psi average injection pressure \\
\hline 1030 & 1 & 2 & & 25 & 65 psi average injection pressure \\
\hline 1028 & 1 & $\overline{4}$ & & 25 & 65 psi average injection pressure \\
\hline 1030 & 1 & $\overline{3}$ & & 25 & 65 psi average injection pressure \\
\hline 1028 & 1 & 5 & & 25 & 65 psi average injection pressure \\
\hline 1030 & 1 & 4 & & 25 & 65 psi average injection pressure \\
\hline 1028 & 1 & 6 & & 25 & 65 psi average injection pressure \\
\hline 1030 & 1 & $\overline{5}$ & & 25 & 65 psi average injection pressure \\
\hline 1028 & 1 & 7 & & 25 & 65 psi average injection pressure \\
\hline 1030 & 1 & 6 & & 25 & 65 psi average injection pressure \\
\hline 1030 & 1 & 7 & & 25 & 65 psi average injection pressure \\
\hline 1031 & 1 & 2 & & 25 & 65 psi average injection pressure \\
\hline 1032 & 1 & $\overline{3}$ & & 25 & 65 psi average injection pressure \\
\hline 1031 & 1 & 3 & & 25 & 65 psi average injection pressure \\
\hline 1032 & 1 & 4 & & 25 & 65 psi average injection pressure \\
\hline 1031 & 1 & 4 & & 25 & 65 psi average injection pressure \\
\hline 1032 & 1 & 5 & & 25 & 65 psi average injection pressure \\
\hline 1031 & 1 & 5 & & 25 & 65 psi average iniection pressure \\
\hline 1032 & 1 & 6 & & 25 & 65 psi average injection pressure \\
\hline 1031 & 1 & 6 & & 25 & 65 psi average injection pressure \\
\hline 1032 & 1 & 7 & & 25 & 65 psi average injection pressure \\
\hline 1033 & $T$ & 2 & & 25 & 65 psi average injection pressure \\
\hline 1034 & 1 & 2 & & 25 & 65 psi average injection pressure \\
\hline
\end{tabular}




\section{ACT REMBCO JOINT VENTURE \\ Ornl Wag 4 Seeps 4 \& 6 Removal Action Project}

Casing Grout Table

Date: August 06, 1996

\begin{tabular}{|c|c|c|c|c|c|}
\hline $\begin{array}{l}\text { Hole } \\
\text { No. }\end{array}$ & $\begin{array}{c}\text { Trench } \\
\text { No. }\end{array}$ & $\begin{array}{c}\text { Sleeve } \\
\text { No. }\end{array}$ & $\begin{array}{l}\text { Depth of injection } \\
\text { From Ground Sur. } \\
\text { (Feet) }\end{array}$ & $\begin{array}{c}\text { Casing Grout } \\
\text { Quantity Injected } \\
\text { (Litres) }\end{array}$ & Comments \\
\hline 1033 & 1 & $\overline{3}$ & & 25 & 65 psi average injection pressure \\
\hline 1034 & 1 & 3 & & 25 & 65 psi average injection pressure \\
\hline 1033 & 1 & 4 & & 25 & 65 psi average injection pressure \\
\hline 1034 & 1 & 4 & & 25 & 65 psi average injection pressure \\
\hline 1033 & 1 & 5 & & 25 & 65 psi average injection pressure \\
\hline 1034 & 1 & 5 & & 25 & 65 psi average injection pressure \\
\hline 1033 & $\overline{1}$ & 6 & & 25 & 65 psi average injection pressure \\
\hline 1034 & 1 & 6 & & 25 & 65 psi average injection pressure \\
\hline 1033 & 1 & 7 & & 25 & 65 psi average injection pressure \\
\hline 1034 & 1 & 7 & & 25 & 65 psi average injection pressure \\
\hline 1035 & 1 & 2 & & 25 & 65 psi average injection pressure \\
\hline 1036 & 1 & 2 & & 25 & 65 psi average injection pressure \\
\hline 1035 & 1 & $\overline{3}$ & & 25 & 65 psi average injection pressure \\
\hline 1036 & 1 & 3 & & 25 & 65 psi average injection pressure \\
\hline 1035 & 1 & 4 & & 25 & 65 psi average injection pressure \\
\hline 1036 & 1 & 4 & & 25 & 65 psi average injection pressure \\
\hline 1035 & 1 & 5 & & 25 & 65 psi average injection pressure \\
\hline 1036 & 1 & 5 & & 25 & 65 psi average injection pressure \\
\hline 1035 & 1 & 6 & & 25 & 65 psi average injection pressure \\
\hline 1036 & 1 & 6 & & 25 & 65 psi average injection pressure \\
\hline 1035 & 1 & 7 & & 25 & 65 psi average injection pressure \\
\hline 1036 & 1 & 7 & & 25 & 65 psi average injection pressure \\
\hline 1037 & 1 & 2 & & 25 & 65 psi average injection pressure \\
\hline 1038 & 1 & 2 & & 25 & 65 psi average injection pressure \\
\hline 1037 & 1 & $\overline{3}$ & & 25 & 65 psi average injection pressure \\
\hline 1038 & 1 & 3 & & 25 & 65 psi average injection pressure \\
\hline 1037 & $\overline{1}$ & $\overline{4}$ & & 25 & 65 psi average injection pressure \\
\hline 1038 & 1 & 4 & & 25 & 65 psi average injection pressure \\
\hline 1037 & 1 & 5 & & 25 & 65 psi average injection pressure \\
\hline 1038 & 1 & 5 & & 25 & 65 psi average injection pressure \\
\hline 1037 & 1 & 6 & & 25 & 65 psi average injection pressure \\
\hline 1038 & 1 & 6 & & 25 & 65 psi average injection pressure \\
\hline 1037 & 1 & $\frac{7}{7}$ & & 25 & 65 psi average injection pressure \\
\hline
\end{tabular}




\title{
ACT REMBCO JOINT VENTURE \\ Ornl Wag 4 Seeps 4 \& 6 Removal Action Project
}

\author{
Casing Grout Table
}

Date: August 07, 1996

\begin{tabular}{|c|c|c|c|c|}
\hline $\begin{array}{l}\text { Hole } \\
\text { No. }\end{array}$ & $\begin{array}{c}\text { Trench } \\
\text { No. }\end{array}$ & $\begin{array}{c}\text { Sleeve } \\
\text { No. }\end{array}$ & $\begin{array}{l}\text { Casing Grout } \\
\text { Quantity Injected } \\
\text { (Litres) }\end{array}$ & Comments \\
\hline 1039 & $\overline{1}$ & 2 & 25 & 50 psi average injection pressure \\
\hline 1040 & 1 & 2 & 25 & 50 psi average injection pressure \\
\hline 1039 & 1 & $\overline{3}$ & 25 & 50 psi average injection pressure \\
\hline 1040 & 1 & 3 & 25 & 50 psi average injection pressure \\
\hline 1039 & $\overline{1}$ & 4 & 25 & $50 \mathrm{psi}$ average injection pressure \\
\hline 1040 & 1 & 4 & 25 & 50 osi average injection pressure \\
\hline 1039 & 1 & 5 & 25 & 50 psi average injection pressure \\
\hline 1040 & 1 & 5 & 25 & 50 psi average injection pressure \\
\hline 1039 & 1 & 6 & 25 & 50 psi average injection pressure \\
\hline 1040 & 1 & 6 & 25 & 50 psi average injection pressure \\
\hline 1039 & 1 & 7 & 25 & 50 psi average injection pressure \\
\hline 1041 & 1 & 2 & 25 & 50 psi average injection pressure \\
\hline 1042 & 1 & 2 & 25 & 50 psi average injection pressure \\
\hline 1041 & 1 & 3 & 25 & $50 \mathrm{psi}$ average injection pressure \\
\hline 1042 & 1 & 3 & 25 & 50 psi average injection pressure \\
\hline 1041 & 1 & 4 & 25 & 50 psi average injection pressure \\
\hline 1042 & 1 & 4 & 25 & 50 psi average injection pressure \\
\hline 1041 & 1 & 5 & 25 & 50 psi average injection pressure \\
\hline 1042 & 1 & 5 & 25 & 50 psi average injection pressure \\
\hline 1043 & 1 & 2 & 25 & 50 psi average injection pressure \\
\hline 1044 & 1 & $\frac{\pi}{2}$ & 25 & 50 psi average injection pressure \\
\hline 1043 & $\frac{1}{1}$ & 3 & 25 & 50 psi average injection pressure \\
\hline 1044 & 1 & 3 & 25 & 50 psi average injection pressure \\
\hline 1043 & $\frac{i}{1}$ & 4 & 25 & 50 psi average injection pressure \\
\hline 1044 & 1 & $\overline{4}$ & 25 & 50 psi average injection pressure \\
\hline 1043 & 1 & 5 & 25 & $50 \mathrm{psi}$ average injection pressure \\
\hline 1044 & 1 & 5 & 25 & 50 psi average injection pressure \\
\hline 1043 & 1 & 6 & 25 & 50 psi average injection oressure \\
\hline 1044 & 1 & 6 & 25 & 50 psi average injection pressure \\
\hline 1043 & 1 & 7 & 25 & 50 psi average injection pressure \\
\hline 1044 & 1 & 7 & 25 & 50 psi average injection pressure \\
\hline
\end{tabular}




\title{
ACT REMBCO JOINT VENTURE \\ Ornl Wag 4 Seeps 4 \& 6 Removal Action Project
}

\author{
Casing Grout Table
}

Date: August 07, 1996

\begin{tabular}{|c|c|c|c|l|}
\hline $\begin{array}{c}\text { Hole } \\
\text { No. }\end{array}$ & $\begin{array}{c}\text { Trench } \\
\text { No. }\end{array}$ & $\begin{array}{c}\text { Sleeve } \\
\text { No. }\end{array}$ & $\begin{array}{c}\text { Casing Grout } \\
\text { Quantity Injected } \\
\text { (Litres) }\end{array}$ & Comments \\
\hline 1045 & 1 & 2 & 25 & 50 psi average injection pressure \\
\hline 1046 & 1 & 2 & 25 & 50 psi average injection pressure \\
\hline 1045 & 1 & 3 & 25 & 50 psi average injection pressure \\
\hline 1046 & 1 & 3 & 25 & 50 psi average injection pressure \\
\hline 1045 & 1 & 4 & 25 & 50 psi average injection pressure \\
\hline 1046 & 1 & 4 & 25 & 50 psi average injection pressure \\
\hline 1045 & 1 & 5 & 25 & 50 psi average injection pressure \\
\hline 1046 & 1 & 5 & 25 & 50 psi average injection pressure \\
\hline 1045 & 1 & 6 & 25 & 50 psi average injection pressure \\
\hline 1046 & 1 & 6 & 25 & 50 psi average injection pressure \\
\hline 1045 & 1 & 7 & 25 & 50 psi average injection pressure \\
\hline 1046 & 1 & 7 & 25 & 50 psi average injection pressure \\
\hline 1047 & 1 & 2 & 25 & 50 psi average injection pressure \\
\hline 1049 & 1 & 2 & 25 & 50 psi average injection pressure \\
\hline 1047 & 1 & 3 & 25 & 50 psi average injection pressure \\
\hline 1049 & 1 & 3 & 25 & 50 psi average injection pressure \\
\hline 1047 & 1 & 4 & 25 & 50 psi average injection pressure \\
\hline 1049 & 1 & 4 & 25 & 50 psi average injection pressure \\
\hline 1047 & 1 & 5 & 25 & 50 psi average injection pressure \\
\hline 1049 & 1 & 5 & 25 & 50 psi average injection pressure \\
\hline 1047 & 1 & 6 & 25 & 50 psi average injection pressure \\
\hline 1049 & 1 & 6 & 25 & 50 psi average injection pressure \\
\hline 1049 & 1 & 7 & 25 & 50 psi average injection pressure \\
\hline 1048 & 1 & 2 & 25 & 55 psi average injection pressure \\
\hline 1050 & 1 & 2 & 25 & 55 psi average injection pressure \\
\hline 1048 & 1 & 3 & 25 & 55 psi average injection pressure \\
\hline 1050 & 1 & 3 & 25 & 55 psi average injection pressure \\
\hline 1048 & 1 & 4 & 25 & 55 psi average injection pressure \\
\hline 1050 & 1 & 4 & 25 & 55 psi average injection pressure \\
\hline 1048 & 1 & 5 & 25 & 55 psi average injection pressure \\
\hline 1050 & 1 & 5 & 25 & 55 psi average injection pressure \\
\hline
\end{tabular}




\title{
ACT REMBCO JOINT VENTURE \\ Ornl Wag 4 Seeps 4 \& 6 Removal Action Project
}

\author{
Casing Grout Tabie
}

Date: August 07, 1996

\begin{tabular}{|c|c|c|c|c|}
\hline $\begin{array}{l}\text { Hole } \\
\text { No. }\end{array}$ & $\begin{array}{c}\text { Trench } \\
\text { No. }\end{array}$ & $\begin{array}{c}\text { Sleeve } \\
\text { No. }\end{array}$ & $\begin{array}{c}\text { Casing Grout } \\
\text { Quantity Injected } \\
\text { (Litres) }\end{array}$ & Comments \\
\hline 1048 & 1 & 6 & 25 & 55 psi average injection pressure \\
\hline 1050 & 1 & 6 & 25 & 55 psi average injection pressure \\
\hline 1048 & 1 & 7 & 25 & 55 psi average injection pressure \\
\hline 1050 & 1 & 7 & 25 & 55 psi average injection pressure \\
\hline 1051 & 1 & 2 & 25 & 55 psi average injection pressure \\
\hline 1052 & 1 & 2 & 25 & 55 psi average injection pressure \\
\hline 1051 & 1 & 3 & 25 & 55 psi average injection pressure \\
\hline 1052 & 1 & 3 & 25 & 55 psi average injection pressure \\
\hline 1051 & 1 & 4 & 25 & 55 psi average injection pressure \\
\hline 1052 & 1 & 4 & 25 & 55 psi average injection pressure \\
\hline 1051 & 1 & 5 & 25 & 55 psi average injection pressure \\
\hline 1052 & 1 & 5 & 25 & 55 psi average injection pressure \\
\hline 1051 & 1 & 6 & 25 & 55 psi average injection pressure \\
\hline 1052 & 1 & 6 & 25 & 55 psi average injection pressure \\
\hline 1051 & 1 & 7 & 25 & 55 psi average injection pressure \\
\hline 1052 & 1 & 7 & 25 & 55 psi average injection pressure \\
\hline 1053 & 1 & 2 & 25 & 55 psi average injection pressure \\
\hline 1054 & 1 & 2 & 25 & 55 psi average injection pressure \\
\hline 1053 & 1 & 3 & 25 & 55 psi average injection pressure \\
\hline 1054 & 1 & 3 & 25 & 55 psi average injection pressure \\
\hline 1053 & 1 & 4 & 25 & 55 psi average injection pressure \\
\hline 1054 & 1 & 4 & 25 & 55 psi average injection pressure \\
\hline 1053 & 1 & 5 & 25 & 55 psi average injection pressure \\
\hline 1054 & 1 & 5 & 25 & 55 psi average injection pressure \\
\hline 1053 & 1 & 6 & 25 & 55 psi average injection pressure \\
\hline 1054 & 1 & 6 & 25 & 55 psi average injection pressure \\
\hline 1053 & 1 & 7 & 25 & 55 psi average injection pressure \\
\hline 1054 & 1 & 7 & 25 & 55 psi average injection pressure \\
\hline 1055 & 1 & 2 & 25 & 55 psi average injection pressure \\
\hline 1057 & 1 & 2 & 25 & 55 psi average injection pressure \\
\hline 1055 & 1 & 3 & 25 & 55 psi average injection pressure \\
\hline
\end{tabular}




\title{
ACT REMBCO JOINT VENTURE Ornl Wag 4 Seeps 4 \& 6 Removal Action Project
}

\author{
Casing Grout Table
}

Date: August 07, 1996

\begin{tabular}{|c|c|c|c|c|}
\hline $\begin{array}{c}\text { Hole } \\
\text { No. }\end{array}$ & $\begin{array}{c}\text { Trench } \\
\text { No. }\end{array}$ & $\begin{array}{c}\text { Sleeve } \\
\text { No. }\end{array}$ & $\begin{array}{c}\text { Casing Grout } \\
\text { Quantity Injected } \\
\text { (Litres) }\end{array}$ & Comments \\
\hline 1057 & 1 & 3 & 25 & 55 psi average injection pressure \\
\hline 1055 & 1 & 4 & 25 & 55 psi average injection pressure \\
\hline 1057 & 1 & 4 & 25 & 55 psi average injection pressure \\
\hline 1055 & 1 & 5 & 25 & 55 psi average injection pressure \\
\hline 1057 & 1 & 5 & 25 & 55 psi average injection pressure \\
\hline 1055 & 1 & 6 & 25 & 55 psi average injection pressure \\
\hline 1057 & 1 & 6 & 25 & 55 psi average injection pressure \\
\hline 1055 & 1 & 7 & 25 & 55 psi average injection pressure \\
\hline 1057 & 1 & 7 & 25 & 55 psi average injection pressure \\
\hline 1062 & 1 & 2 & 25 & 55 psi average injection pressure \\
\hline 1063 & 1 & 2 & 25 & 55 psi average injection pressure \\
\hline 1062 & 1 & 3 & 25 & 55 psi average injection pressure \\
\hline 1063 & 1 & 3 & 25 & 55 psi average injection pressure \\
\hline 1062 & 1 & 4 & 25 & 55 psi average injection pressure \\
\hline 1063 & 1 & 4 & 25 & 55 psi average injection pressure \\
\hline 1062 & 1 & 5 & 25 & 55 psi average injection pressure \\
\hline 1063 & 1 & 5 & 25 & 55 psi average injection pressure \\
\hline 1062 & 1 & 6 & 25 & 55 psi average injection pressure \\
\hline 1063 & 1 & 6 & 25 & 55 psi average injection pressure \\
\hline 1062 & 1 & 7 & 25 & 55 psi average injection pressure \\
\hline 1063 & 1 & 7 & 25 & 55 psi average injection pressure \\
\hline 1065 & 1 & 2 & 25 & 55 psi average injection pressure \\
\hline 1066 & 1 & 2 & 25 & 55 psi average injection pressure \\
\hline 1065 & 1 & 3 & 25 & 55 psi average injection pressure \\
\hline 1066 & 1 & 3 & 25 & 55 psi average injection pressure \\
\hline 1065 & 1 & 4 & 25 & 55 psi average injection pressure \\
\hline 1066 & 1 & 4 & 25 & 55 psi average injection pressure \\
\hline 1065 & 1 & 5 & 25 & 55 psi average injection pressure \\
\hline 1066 & 1 & 5 & 25 & 55 psi average injection pressure \\
\hline 1065 & 1 & 6 & 25 & 55 psi average injection pressure \\
\hline 1066 & 1 & 6 & 25 & 55 psi average injection pressure \\
\hline & & & & \\
\hline
\end{tabular}




\title{
ACT REMBCO JOINT VENTURE Ornl Wag 4 Seeps 4 \& 6 Removal Action Project
}

\author{
Casing Grout Table
}

Date: August 07, 1996

\begin{tabular}{|c|c|c|c|c|}
\hline $\begin{array}{l}\text { Hole } \\
\text { No. }\end{array}$ & $\begin{array}{c}\text { Trench } \\
\text { No. }\end{array}$ & $\begin{array}{c}\text { Sleeve } \\
\text { No. }\end{array}$ & $\begin{array}{c}\text { Casing Grout } \\
\text { Quantity Injected } \\
\text { (Litres) }\end{array}$ & Comments \\
\hline 1065 & 1 & 7 & 25 & 55 psi average injection pressure \\
\hline 1066 & 1 & 7 & 25 & 55 psi average injection pressure \\
\hline 1067 & 1 & 2 & 25 & 55 psi average injection pressure \\
\hline 1068 & 1 & 2 & 25 & 55 psi average injection pressure \\
\hline 1067 & $\overline{1}$ & $\overline{3}$ & 25 & 55 psi average injection pressure \\
\hline 1068 & 1 & 3 & 25 & 55 psi average injection pressure \\
\hline 1067 & 1 & 4 & 25 & 55 psi average injection pressure \\
\hline 1068 & 1 & 4 & 25 & 55 psi average injection pressure \\
\hline 1067 & 1 & 5 & 25 & 55 psi average injection pressure \\
\hline 1068 & 1 & 5 & 25 & 55 psi average injection pressure \\
\hline 1067 & 1 & 6 & 25 & 55 psi average injection pressure \\
\hline 1068 & $\overline{1}$ & 6 & 25 & 55 psi average injection pressure \\
\hline 1067 & 1 & 7 & 25 & 55 psi average injection pressure \\
\hline 1068 & 1 & 7 & 25 & 55 psi average injection pressure \\
\hline 1069 & 1 & 2 & 25 & 65 psi average injection pressure \\
\hline 1070 & 1 & 2 & 25 & 65 psi average injection pressure \\
\hline 1069 & 1 & 3 & 25 & 65 psi average injection pressure \\
\hline 1070 & 1 & 3 & 25 & 65 psi average injection pressure \\
\hline 1069 & 1 & 4 & 25 & 65 psi average injection pressure \\
\hline 1070 & $\overline{1}$ & 4 & 25 & 65 psi average injection pressure \\
\hline 1069 & 1 & 5 & 25 & 65 psi average injection pressure \\
\hline 1070 & 1 & 5 & 25 & 65 psi average injection pressure \\
\hline 1069 & 1 & 6 & 25 & 65 psi average injection pressure \\
\hline 1070 & 1 & 6 & 25 & 65 psi average injection pressure \\
\hline 1069 & 1 & 7 & 25 & 65 psi average injection pressure \\
\hline 1070 & 1 & 7 & 25 & 65 psi average injection pressure \\
\hline 1060 & 1 & 2 & 25 & 65 psi average injection pressure \\
\hline 1061 & 1 & 2 & 25 & 65 psi average injection pressure \\
\hline 1060 & 1 & 3 & 25 & 65 psi average injection pressure \\
\hline 1061 & 1 & 3 & 25 & 65 psi average injection pressure \\
\hline 1060 & 1 & $\overline{4}$ & 25 & 65 psi average injection pressure \\
\hline
\end{tabular}




\title{
ACT REMBCO JOINT VENTURE \\ Ornl Wag 4 Seeps 4 \& 6 Removal Action Project
}

\author{
Casing Grout Table
}

Date: August 07, 1996

\begin{tabular}{|c|c|c|c|c|}
\hline $\begin{array}{l}\text { Hole } \\
\text { No. }\end{array}$ & $\begin{array}{c}\text { Trench } \\
\text { No. }\end{array}$ & $\begin{array}{c}\text { Sleeve } \\
\text { No. }\end{array}$ & $\begin{array}{c}\text { Casing Grout } \\
\text { Quantity injected } \\
\text { (Litres) }\end{array}$ & Comments \\
\hline 1061 & 1 & 4 & 25 & 65 psi average injection pressure \\
\hline 1060 & 1 & 5 & 25 & 65 psi average injection pressure \\
\hline 1061 & 1 & 5 & 25 & 65 psi average injection pressure \\
\hline 1060 & 1 & 6 & 25 & 65 psi average injection pressure \\
\hline 1061 & 1 & 6 & 25 & 65 psi average injection pressure \\
\hline 1060 & 1 & 7 & 25 & 65 psi average injection pressure \\
\hline 1061 & 1 & 7 & 25 & 65 psi average injection pressure \\
\hline 1058 & 1 & 2 & 25 & 65 psi average injection pressure \\
\hline 1059 & 1 & 2 & 25 & 65 psi average injection pressure \\
\hline 1058 & 1 & 3 & 25 & 65 psi average injection pressure \\
\hline 1059 & 1 & 3 & 25 & 65 psi average injection pressure \\
\hline 1058 & 1 & 4 & 25 & 65 psi average injection pressure \\
\hline 1059 & 1 & 4 & 25 & 65 psi average injection pressure \\
\hline 1058 & 1 & 5 & 25 & 65 psi average injection pressure \\
\hline 1059 & 1 & 5 & 25 & 65 psi average injection pressure \\
\hline 1058 & 1 & 6 & 25 & 65 psi average injection pressure \\
\hline 1059 & 1 & 6 & 25 & 65 psi average injection pressure \\
\hline 1058 & 1 & 7 & 25 & 65 psi average injection pressure \\
\hline 1059 & 1 & 7 & 25 & 65 psi average injection pressure \\
\hline 1056 & 1 & 2 & 25 & 65 psi average injection pressure \\
\hline 1056 & 1 & 3 & 25 & 65 psi average injection pressure \\
\hline 1056 & 1 & 4 & 25 & 65 psi average injection pressure \\
\hline 1056 & 1 & 5 & 25 & 65 psi average injection pressure \\
\hline 1056 & 1 & 6 & 25 & 65 psi average injection pressure \\
\hline 1056 & 1 & 7 & 25 & 65 psi average injection pressure \\
\hline
\end{tabular}


ACT REMBCO JOINT VENTURE

Ornl Wag 4 Seeps 4 \& 6 Removal Action Project

Casing Grout Table

August 09, 1996

\begin{tabular}{|c|c|c|c|c|}
\hline $\begin{array}{l}\text { Hole } \\
\text { No. }\end{array}$ & $\begin{array}{c}\text { Trench } \\
\text { No. }\end{array}$ & $\begin{array}{c}\text { Sleeve } \\
\text { No. }\end{array}$ & $\begin{array}{c}\text { Casing Grout } \\
\text { Quantity Injected } \\
\text { (Litres) }\end{array}$ & Comments \\
\hline 1210 & 1 & 1,2 & 50 & 75 psi average injection pressure \\
\hline 1210 & 1 & 3 & 25 & 75 psi average injection pressure \\
\hline 1210 & 1 & 4 & 25 & 75 psi average injection pressure \\
\hline 1210 & 1 & 5 & 25 & 75 psi average injection pressure \\
\hline 1210 & 1 & 6 & 25 & 75 psi average injection pressure \\
\hline 1210 & 1 & 7 & 25 & 75 psi average injection pressure \\
\hline 1213 & 1 & 1,2 & 50 & 75 psi average injection pressure \\
\hline 1213 & 1 & 3 & 25 & 75 psi average injection pressure \\
\hline 1213 & 1 & 4 & 25 & 75 psi average injection pressure \\
\hline 1213 & 1 & 5 & 25 & 75 psi average injection pressure \\
\hline 1213 & 1 & 6 & 25 & 75 psi average injection pressure \\
\hline 1213 & 1 & 7 & 25 & 75 psi average injection pressure \\
\hline 1213 & 1 & 8 & 25 & 75 psi average injection pressure \\
\hline 1213 & 1 & 9 & 25 & 75 psi average injection pressure \\
\hline 1206 & 1 & $\overline{1,2}$ & 50 & 75 psi average injection pressure \\
\hline 1206 & 1 & 3 & 25 & 75 psi average injection pressure \\
\hline 1206 & 1 & 4 & 25 & 75 psi average injection pressure \\
\hline 1206 & 1 & 5 & 25 & 75 psi average injection pressure \\
\hline 1206 & 1 & 6 & 25 & 75 psi average injection pressure \\
\hline 1206 & 1 & 7 & 25 & 75 psi average injection pressure \\
\hline 1206 & 1 & 8 & 25 & 75 psi average injection pressure \\
\hline 1211 & 1 & 1,2 & 50 & 75 psi average injection pressure \\
\hline 1211 & 1 & 3 & 25 & 75 psi average injection pressure \\
\hline 1211 & 1 & 4 & 25 & 75 psi average injection pressure \\
\hline 1211 & 1 & 5 & 25 & 75 psi average injection pressure \\
\hline 1211 & 1 & 6 & 25 & 75 psi average injection pressure \\
\hline 1211 & 1 & 7 & 25 & 75 psi average injection pressure \\
\hline 1211 & 1 & 8 & 25 & 75 psi average injection pressure \\
\hline 1211 & 1 & 9 & 25 & 75 psi average injection pressure \\
\hline 1214 & 1 & 1,2 & 50 & 75 psi average injection pressure \\
\hline 1214 & 1 & 3 & 25 & 75 psi average injection pressure \\
\hline 1214 & 1 & 4 & 25 & 75 psi average injection pressure \\
\hline 1214 & 1 & 5 & 25 & 75 psi average injection pressure \\
\hline 1205 & 1 & 1,2 & 50 & 75 psi average injection pressure \\
\hline
\end{tabular}


ACT REMBCO JOINT VENTURE

Orni Wag 4 Seeps 4 \& 6 Removal Action Project

Casing Grout Table

August 09, 1996

\begin{tabular}{|c|c|c|c|c|}
\hline $\begin{array}{c}\text { Hole } \\
\text { No. }\end{array}$ & $\begin{array}{c}\text { Trench } \\
\text { No. }\end{array}$ & $\begin{array}{c}\text { Sleeve } \\
\text { No. }\end{array}$ & $\begin{array}{c}\text { Casing Grout } \\
\text { Quantity Injected } \\
\text { (Litres) }\end{array}$ & Comments \\
\hline 1205 & 1 & 3 & 25 & 75 psi average injection pressure \\
\hline 1205 & 1 & 4 & 25 & 75 psi average injection pressure \\
\hline 1212 & 1 & 1,2 & 50 & 75 psi average injection pressure \\
\hline 1212 & 1 & 3 & 25 & 75 psi average injection pressure \\
\hline 1212 & 1 & 4 & 25 & 75 psi average injection pressure \\
\hline 1212 & 1 & 5 & 25 & 75 psi average injection pressure \\
\hline 1212 & 1 & 6 & 25 & 75 psi average injection pressure \\
\hline 1212 & 1 & 7 & 25 & 75 psi average injection pressure \\
\hline 1212 & 1 & 8 & 25 & 75 psi average injection pressure \\
\hline 1212 & 1 & 9 & 25 & 75 psi average injection pressure \\
\hline 1212 & 1 & 10 & 25 & 75 psi average injection pressure \\
\hline
\end{tabular}

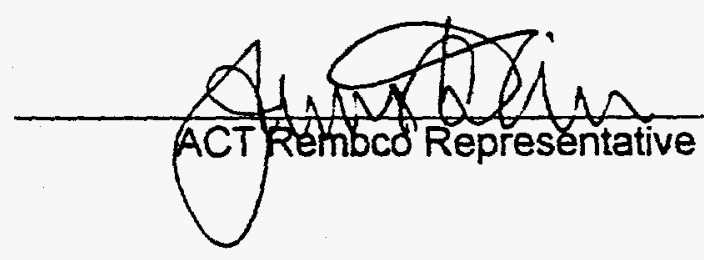




\section{ACT REMBCO JOINT VENTURE \\ Ornl Wag 4 Seeps 4 \& 6 Removal Action Project}

Casing Grout Table

September 06, 1996

\begin{tabular}{|c|c|c|c|l|}
\hline $\begin{array}{c}\text { Hole } \\
\text { No. }\end{array}$ & $\begin{array}{c}\text { Trench } \\
\text { No. }\end{array}$ & $\begin{array}{c}\text { Sleeve } \\
\text { No. }\end{array}$ & $\begin{array}{c}\text { Casing Grout } \\
\text { Quantity Injected } \\
\text { Litres) }\end{array}$ & Comments \\
\hline 1071 & 1 & $1-7$ & 140 & $60 \mathrm{psi}$ average injection pressure \\
\hline 1072 & 1 & $1-7$ & 140 & $60 \mathrm{psi}$ average injection pressure \\
\hline 1073 & 1 & $1-7$ & 140 & $60 \mathrm{psi}$ average injection pressure \\
\hline 1074 & 1 & $1-7$ & 140 & $60 \mathrm{psi}$ average injection pressure \\
\hline 1075 & 1 & $1-7$ & 140 & $60 \mathrm{psi}$ average injection pressure \\
\hline 1076 & 1 & $1-7$ & 140 & $60 \mathrm{psi}$ average injection pressure \\
\hline 1077 & 1 & $1-7$ & 140 & $60 \mathrm{psi}$ average injection pressure \\
\hline 1078 & 1 & $1-7$ & 140 & $60 \mathrm{psi}$ average injection pressure \\
\hline 1079 & 1 & $1-7$ & 140 & $60 \mathrm{psi}$ average injection pressure \\
\hline 1081 & 1 & $1-7$ & 140 & $60 \mathrm{psi}$ average injection pressure \\
\hline 1082 & 1 & $1-7$ & 140 & $60 \mathrm{psi}$ average injection pressure \\
\hline 1083 & 1 & $1-7$ & 140 & $60 \mathrm{psi}$ average injection pressure \\
\hline 2001 & 2 & $1-7$ & 140 & $60 \mathrm{psi}$ average injection pressure \\
\hline 2002 & 2 & $1-7$ & 140 & $60 \mathrm{psi}$ average injection pressure \\
\hline 2003 & 2 & $1-7$ & 140 & $60 \mathrm{psi}$ average injection pressure \\
\hline 2004 & 2 & $1-7$ & 140 & $60 \mathrm{psi}$ average injection pressure \\
\hline 2005 & 2 & $1-7$ & 140 & $60 \mathrm{psi}$ average injection pressure \\
\hline 2006 & 2 & $1-7$ & 140 & $60 \mathrm{psi}$ average injection pressure \\
\hline 2007 & 2 & $1-7$ & 140 & $60 \mathrm{psi}$ average injection pressure \\
\hline 2034 & 2 & $1-7$ & 140 & $60 \mathrm{psi}$ average injection pressure \\
\hline 2035 & 2 & $1-7$ & 140 & $60 \mathrm{psi}$ average injection pressure \\
\hline 2036 & 2 & $1-7$ & 140 & $60 \mathrm{psi}$ average injection pressure \\
\hline 2037 & 2 & $1-7$ & 140 & $60 \mathrm{psi}$ average injection pressure \\
\hline 2038 & 2 & $1-7$ & 140 & $60 \mathrm{psi}$ average injection pressure \\
\hline 2039 & 2 & $1-7$ & 140 & $60 \mathrm{psi}$ average injection pressure \\
\hline & & & & \\
\hline
\end{tabular}




\section{ACT REMBCO JOINT VENTURE \\ Ornl Wag 4 Seeps 4 \& 6 Removal Action Project}

\section{Casing Grout Table}

September 07, 1996

\begin{tabular}{|c|c|c|c|l|}
\hline $\begin{array}{c}\text { Hole } \\
\text { No. }\end{array}$ & $\begin{array}{c}\text { Trench } \\
\text { No. }\end{array}$ & $\begin{array}{c}\text { Sleeve } \\
\text { No. }\end{array}$ & $\begin{array}{c}\text { Casing Grout } \\
\text { Quantity Injected } \\
\text { (Litres) }\end{array}$ & Comments \\
\hline 1080 & 1 & $1-5$ & 100 & 60 psi average injection pressure \\
\hline 2008 & 2 & $1-7$ & 140 & 60 psi average injection pressure \\
\hline 2009 & 2 & $1-7$ & 140 & 60 psi average injection pressure \\
\hline 2010 & 2 & $1-3$ & 60 & 60 psi average injection pressure \\
\hline 2011 & 2 & $1-3$ & 60 & 60 psi average injection pressure \\
\hline 2012 & 2 & $1-3$ & 60 & 60 psi average injection pressure \\
\hline 2013 & 2 & $1-7$ & 140 & 60 psi average injection pressure \\
\hline 2018 & 2 & $1-7$ & 140 & 60 psi average injection pressure \\
\hline 2022 & 2 & $1-7$ & 140 & 60 psi average injection pressure \\
\hline 2023 & 2 & $1-7$ & 140 & 60 psi average injection pressure \\
\hline 2024 & 2 & $1-7$ & 140 & 60 psi average injection pressure \\
\hline 2025 & 2 & $1-5$ & 100 & 60 psi average injection pressure \\
\hline 2026 & 2 & $1-7$ & 140 & 60 psi average injection pressure \\
\hline 2029 & 2 & $1-9$ & 180 & 60 psi average injection pressure \\
\hline 2030 & 2 & $1-3$ & 60 & 55 psi average injection pressure \\
\hline 2031 & 2 & $1-9$ & 180 & 55 psi average injection pressure \\
\hline 2040 & 2 & $1-9$ & 180 & 55 psi average injection pressure \\
\hline 2041 & 2 & $1-9$ & 180 & 55 psi average injection pressure \\
\hline 2042 & 2 & $1-7$ & 140 & 55 psi average injection pressure \\
\hline 2043 & 2 & $1-7$ & 140 & 55 psi average injection pressure \\
\hline 2044 & 2 & $1-7$ & 140 & 55 psi average injection pressure \\
\hline 2045 & 2 & $1-9$ & 180 & 55 psi average injection pressure \\
\hline 2046 & 2 & $1-7$ & 140 & 55 psi average injection pressure \\
\hline 2047 & 2 & $1-7$ & 140 & 55 psi average injection pressure \\
\hline & & & & \\
\hline
\end{tabular}


ACT REMBCO JOINT VENTURE

Ornl Wag 4 Seeps 4 \& 6 Removal Action Project

Casing Grout Table

September 11, 1996

\begin{tabular}{|c|c|c|c|c|}
\hline $\begin{array}{l}\text { Hole } \\
\text { No. }\end{array}$ & $\begin{array}{c}\text { Trench } \\
\text { No. }\end{array}$ & $\begin{array}{c}\text { Sleeve } \\
\text { No. }\end{array}$ & $\begin{array}{c}\text { Casing Grout } \\
\text { Quantity Injected } \\
\text { (Litres) }\end{array}$ & Comments \\
\hline 4001 & 4 & 1.7 & 140 & 70 psi average injection pressure \\
\hline 4002 & 4 & $1-7$ & 140 & 70 psi average injection pressure \\
\hline 4003 & 4 & $1-7$ & 140 & 70 psi average injection pressure \\
\hline 4004 & 4 & $1-5$ & 100 & 70 psi average injection pressure \\
\hline 4005 & 4 & $1-5$ & 100 & 70 psi average injection pressure \\
\hline 4006 & 4 & 15 & 100 & 70 psi average injection pressure \\
\hline 4007 & 4 & $1-5$ & 100 & 70 psi average injection pressure \\
\hline 4008 & 4 & 1.5 & 100 & 70 psi average injection pressure \\
\hline 4009 & $\overline{4}$ & $1-5$ & 100 & $70 \mathrm{psi}$ average injection pressure \\
\hline 4010 & 4 & $1-5$ & 100 & 70 psi average injection pressure \\
\hline 4011 & 4 & $1-3$ & 60 & $70 \mathrm{psi}$ average injection pressure \\
\hline 4012 & 4 & $1-3$ & 60 & $70 \mathrm{psi}$ average injection pressure \\
\hline 4013 & 4 & $1-5$ & 100 & 70 psi average injection pressure \\
\hline 4014 & $\overline{4}$ & $1-3$ & 60 & 70 psi average injection pressure \\
\hline 4015 & $\overline{4}$ & $1-3$ & 60 & $70 \mathrm{psi}$ average injection pressure \\
\hline 4016 & $\overline{4}$ & $1-5$ & 100 & 70 psi average injection pressure \\
\hline 4017 & $\overline{4}$ & $1-5$ & 100 & 70 psi average injection pressure \\
\hline 4018 & 4 & $1-5$ & 100 & $70 \mathrm{psi}$ average injection pressure \\
\hline 4019 & 4 & $1-5$ & 100 & 70 psi average injection pressure \\
\hline 4020 & 4 & $1-3$ & 60 & $70 \mathrm{psi}$ average injection pressure \\
\hline 4021 & $\overline{4}$ & $1-5$ & 100 & $70 \mathrm{psi}$ average injection pressure \\
\hline 4022 & 4 & $1-3$ & 60 & $70 \mathrm{psi}$ average injection pressure \\
\hline 4023 & 4 & $1-5$ & 100 & 70 psi average injection pressure \\
\hline 4025 & 4 & $1-3$ & 60 & $70 \mathrm{psi}$ average injection pressure \\
\hline 4026 & 4 & $1-5$ & 100 & $70 \mathrm{psi}$ average injection pressure \\
\hline 4028 & 4 & $1-5$ & 100 & 70 psi average injection pressure \\
\hline 4031 & 4 & $1-5$ & 100 & $70 \mathrm{psi}$ average injection pressure \\
\hline 4032 & 4 & $1-3$ & 60 & 70 psi average injection pressure \\
\hline
\end{tabular}


ACT REMBCO JOINT VENTURE

Ornl Wag 4 Seeps 4 \& 6 Removal Action Project

\section{Casing Grout Table}

\section{Sentemicer 1. 19g6}

\begin{tabular}{|c|c|c|c|c|}
\hline $\begin{array}{c}\text { Hole } \\
\text { No. }\end{array}$ & $\begin{array}{c}\text { Trench } \\
\text { No. }\end{array}$ & $\begin{array}{c}\text { Sleeve } \\
\text { No. }\end{array}$ & $\begin{array}{c}\text { Casing Grout } \\
\text { Quantity Injected } \\
\text { (Litres) }\end{array}$ & Comments \\
\hline 4033 & 4 & $1-3$ & 100 & 70 psi average injection pressure \\
\hline 4034 & 4 & $1-3$ & 60 & 70 psi average injection pressure \\
\hline 4036 & 4 & $1-5$ & 100 & 70 psi average injection pressure \\
\hline 4038 & 4 & $1-3$ & 60 & 70 psi average injection pressure \\
\hline 4040 & 4 & $1-7$ & 140 & 70 psi average injection pressure \\
\hline 4042 & 4 & $1-7$ & 140 & 70 psi average injection pressure \\
\hline 4046 & 4 & $1-3$ & 60 & 70 psi average injection pressure \\
\hline
\end{tabular}

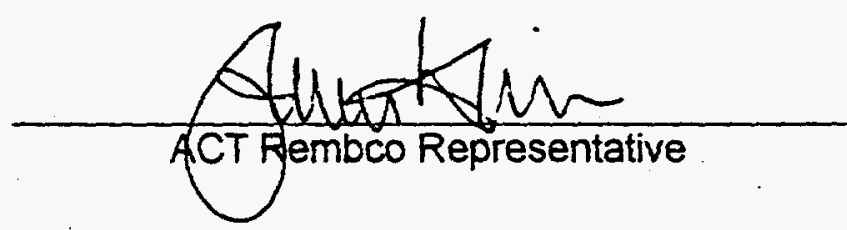




\title{
ACT REMBCO JOINT VENTURE \\ Orni Wag 4 Seeps 4 \& 6 Removal Action Project
}

\author{
Casing Grout Table
}

September 12,1996

\begin{tabular}{|c|c|c|c|l|}
\hline $\begin{array}{c}\text { Hole } \\
\text { No. }\end{array}$ & $\begin{array}{c}\text { Trench } \\
\text { No. }\end{array}$ & $\begin{array}{c}\text { Sleeve } \\
\text { No. }\end{array}$ & $\begin{array}{c}\text { Casing Grout } \\
\text { Quantity Injected } \\
\text { (Litres) }\end{array}$ & Comments \\
\hline 4044 & 4 & $1-7$ & 140 & 70 psi average injection pressure \\
\hline 4045 & 4 & $1-5$ & 100 & 70 psi average injection pressure \\
\hline 4047 & 4 & $1-5$ & 100 & 70 psi average injection pressure \\
\hline 4048 & 4 & $1-7$ & 140 & 70 psi average injection pressure \\
\hline 4050 & 4 & $1-3$ & 60 & 70 psi average injection pressure \\
\hline 4051 & 4 & $1-5$ & 100 & 70 psi average injection pressure \\
\hline 4052 & 4 & $1-7$ & 140 & 70 psi average injection pressure \\
\hline 4053 & 4 & $1-7$ & 140 & 70 psi average injection pressure \\
\hline 4054 & 4 & $1-7$ & 140 & 70 psi average injection pressure \\
\hline 4055 & 4 & $1-5$ & 100 & 70 psi average injection pressure \\
\hline 3001 & 3 & $1-9$ & 180 & 70 psi average injection pressure \\
\hline 3002 & 3 & $1-7$ & 140 & 70 psi average injection pressure \\
\hline 3003 & 3 & $1-7$ & 140 & 70 psi average injection pressure \\
\hline 3004 & 3 & $1-7$ & 140 & 70 psi average injection pressure \\
\hline 3005 & 3 & $1-7$ & 140 & 70 psi average injection pressure \\
\hline 3006 & 3 & $1-5$ & 100 & 70 psi average injection pressure \\
\hline 3007 & 3 & $1-7$ & 140 & 70 psi average injection pressure \\
\hline 3008 & 3 & $1-5$ & 100 & 70 psi average injection pressure \\
\hline 3009 & 3 & $1-7$ & 140 & 70 psi average injection pressure \\
\hline 3010 & 3 & $1-7$ & 140 & 70 psi average injection pressure \\
\hline 3011 & 3 & $1-7$ & 140 & 70 psi average injection pressure \\
\hline 3012 & 3 & $1-9$ & 180 & 70 psi average injection pressure \\
\hline 3013 & 3 & $1-7$ & 140 & 70 psi average injection pressure \\
\hline 3014 & 3 & $1-7$ & 140 & 70 psi average injection pressure \\
\hline 3015 & 3 & $1-7$ & 140 & 70 psi average injection pressure \\
\hline 3016 & 3 & $1-7$ & 140 & 70 psi average injection pressure \\
\hline 3017 & 3 & $1-7$ & 140 & 70 psi average injection pressure \\
\hline 3018 & 3 & $1-11$ & 220 & 70 psi average injection pressure \\
\hline & & & & \\
\hline
\end{tabular}




\title{
ACT REMBCO JOINT VENTURE \\ Ornl Wag 4 Seeps 4 \& 6 Removal Action Project
}

\author{
Casing Grout Table
}

September 12, 1996

\begin{tabular}{|c|c|c|c|c|}
\hline $\begin{array}{c}\text { Hole } \\
\text { No. }\end{array}$ & $\begin{array}{c}\text { Trench } \\
\text { No. }\end{array}$ & $\begin{array}{c}\text { Sleeve } \\
\text { No. }\end{array}$ & $\begin{array}{c}\text { Casing Grout } \\
\text { Quantity Injected } \\
\text { (Litres) }\end{array}$ & Comments \\
\hline 3020 & 3 & $1-7$ & 140 & 70 psi average injection pressure \\
\hline 3022 & 3 & $1-7$ & 140 & 70 psi average injection pressure \\
\hline 3023 & 3 & $1-7$ & 140 & 70 psi average injection pressure \\
\hline 3024 & 3 & $1-7$ & 140 & $70 \mathrm{psi}$ average injection pressure \\
\hline 3025 & 3 & $1-7$ & 140 & 70 psi average injection pressure \\
\hline 3026 & 3 & $1-7$ & 140 & 70 psi average injection pressure \\
\hline 3027 & 3 & $1-7$ & 140 & 70 psi average injection pressure \\
\hline 3028 & 3 & $1-7$ & 140 & 70 psi average injection pressure \\
\hline 3029 & 3 & $1-7$ & 140 & 70 psi average injection pressure \\
\hline 3030 & 3 & $1-7$ & 180 & 70 ss average injection pressure \\
\hline 3031 & 3 & $1-9$ & 180 & $70 \mathrm{psi}$ average injection pressure \\
\hline 3032 & 3 & $1-9$ & 180 & $70 \mathrm{psi}$ average injection pressure \\
\hline 3033 & 3 & $1-7$ & 140 & $70 \mathrm{psi}$ average injection pressure \\
\hline 3034 & 3 & $1-9$ & 180 & $70 \mathrm{psi}$ average injection pressure \\
\hline
\end{tabular}

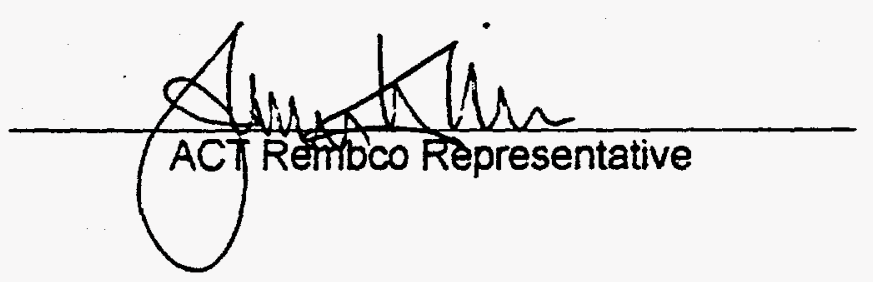




\section{ACT REMBCO JOINT VENTURE \\ Ornl Wag 4 Seeps 4 \& 6 Removal Action Project}

Casing Grout Table

September 13, 1996

\begin{tabular}{|c|c|c|c|c|}
\hline $\begin{array}{l}\text { Hole } \\
\text { No. }\end{array}$ & $\begin{array}{c}\text { Trench } \\
\text { No. }\end{array}$ & $\begin{array}{c}\text { Sleeve } \\
\text { No. }\end{array}$ & $\begin{array}{c}\text { Casing Grout } \\
\text { Quantity Injected } \\
\text { (Litres) }\end{array}$ & Comments \\
\hline 4029 & 4 & $1-3$ & 60 & 60 psi average injection pressure \\
\hline 4030 & 4 & $1-3$ & 60 & 60 psi average injection pressure \\
\hline 4035 & 4 & $1-5$ & 100 & 60 psi average injection pressure \\
\hline 4037 & 4 & $1-5$ & 100 & 60 psi average injection pressure \\
\hline 4039 & 4 & $1-5$ & 100 & 60 psi average injection pressure \\
\hline 4041 & 4 & $1-7$ & 140 & 60 psi average injection pressure \\
\hline 4043 & 4 & $1-5$ & 100 & 60 psi average injection pressure \\
\hline 4049 & 4 & $1-7$ & 140 & 60 psi average injection pressure \\
\hline 4056 & 4 & 1.7 & 140 & $60 \mathrm{psi}$ average injection pressure \\
\hline 3019 & 3 & $1-7$ & 140 & 60 psi average injection pressure \\
\hline 3021 & 3 & $1-7$ & 140 & 60 psi average injection pressure \\
\hline 3035 & 3 & $1-7$ & 140 & $60 \mathrm{psi}$ average injection pressure \\
\hline 3036 & 3 & $1-7$ & 140 & 60 psi average injection pressure \\
\hline 3037 & 3 & $1-7$ & 140 & 60 psi average injection pressure \\
\hline 3038 & 3 & $1-7$ & 140 & 60 psi average injection pressure \\
\hline 3039 & 3 & $1-7$ & 140 & 60 psi average injection pressure \\
\hline 3040 & 3 & $1-5$ & 100 & 60 psi average injection pressure \\
\hline 3041 & 3 & $1-7$ & 140 & 60 psi average injection pressure \\
\hline 3042 & 3 & 1.5 & 100 & 60 psi average injection pressure \\
\hline 3043 & 3 & $1-7$ & 140 & 60 psi average injection pressure \\
\hline 3044 & 3 & $1-7$ & 140 & 60 psi average injection pressure \\
\hline 3045 & 3 & $1-5$ & 100 & $60 \mathrm{psi}$ average injection pressure \\
\hline 3046 & 3 & $1-7$ & 140 & 60 psi average injection pressure \\
\hline 3047 & 3 & $1-9$ & 180 & 60 psi average injection pressure \\
\hline 3048 & 3 & $1-5$ & 100 & 60 psi average injection pressure \\
\hline 3049 & 3 & $1-7$ & 140 & 60 psi average injection pressure \\
\hline 3050 & 3 & $1-5$ & 100 & 60 psi average injection pressure \\
\hline
\end{tabular}



APPENDIX C

IN SITU HYDRAULIC CONDUCTIVITY TESTING RECORDS 


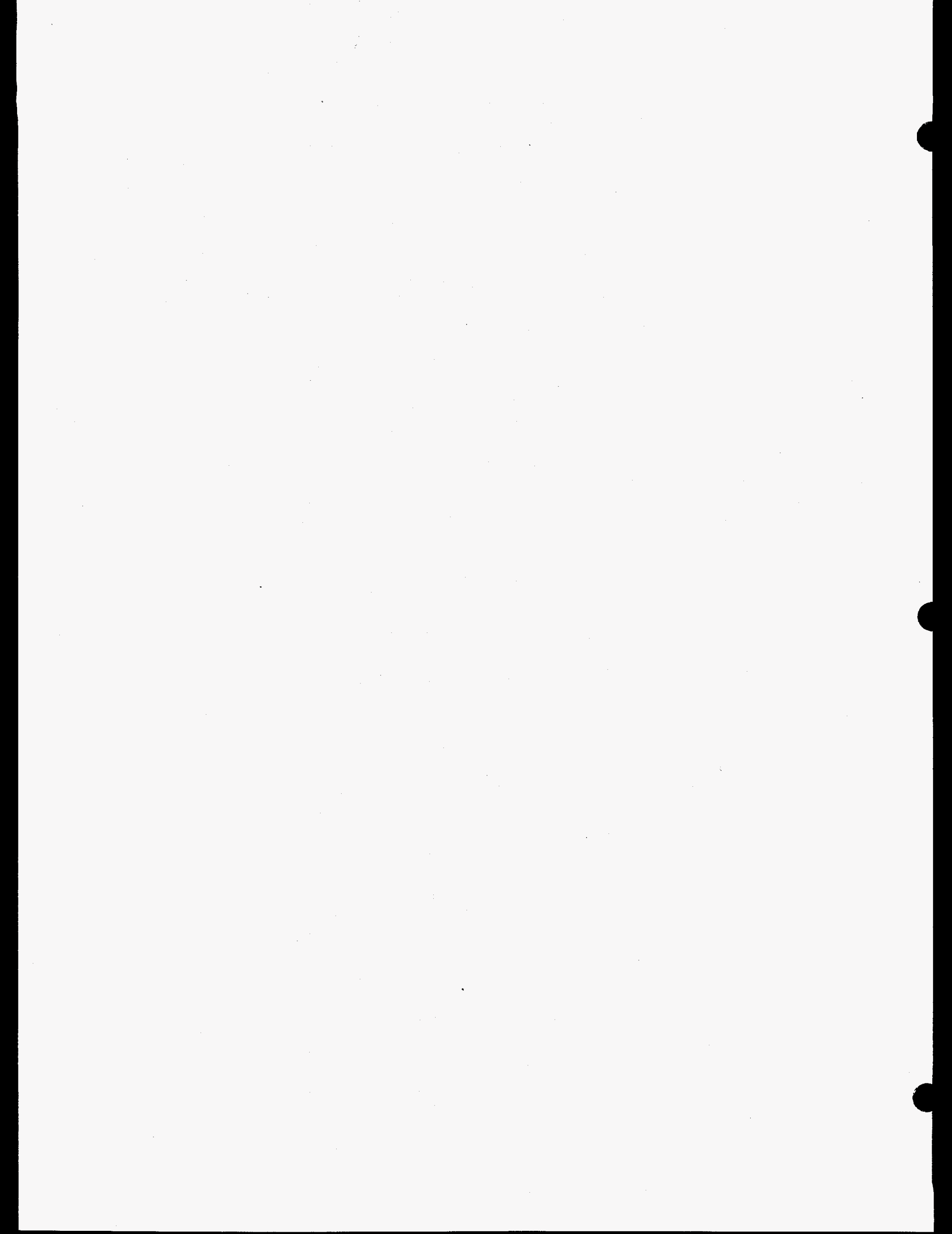




\begin{tabular}{|c|c|c|c|c|c|c|c|c|c|c|c|c|c|c|}
\hline & & & \multirow{2}{*}{\multicolumn{10}{|c|}{ SUMMARY OF RESIDUAL IN SITU HYDRAULIC CONDUCTIVITY CALCULATIONS }} & & \\
\hline & & & & & & & & & & & & & & \\
\hline & & & & & \multicolumn{5}{|c|}{ WAG 4 SEEPS 4 AND 6 REMOVAL ACTION } & & & & & \\
\hline & & & & & \multicolumn{5}{|c|}{ Test Date: October $28 \& 29,1996$} & & & & & \\
\hline & & & & & & & & & & & & & & \\
\hline & & Depth & Depih & & Corr. & & Approx. & & & & & Total & Total & \\
\hline & Stick & to & to Top & Stage & Stage & Avg. Corr. & Depth & Grav. Hd. & Grav. Hd. & Press. & Head & Effec. & Effec. & Hydraulic \\
\hline Hole & Up & Tip & Packer & Length & Length & Flow Rate & to Water & Abv. Grnd. & Bel. Grnd. & Head & Losses & Head & Head & Conductivity \\
\hline No. & (f) & $(f t)$ & (ft) & (ft) & (m) & $(\mathrm{cc} / \mathrm{min})$ & $(\mathrm{ft})^{*}$ & (m) & (m) & (m) & $(m)$ & (m) & (psi) & $(\mathrm{cm} / \mathrm{s})$ \\
\hline 1002 & 1.04 & 12.50 & 6 & 1.899 & 2.04 & 1.0 & 5.2 & 2.53 & 1.585 & 1.76 & 1.42 & 4.455 & 6.337 & $1.45905 \mathrm{E}-07$ \\
\hline 1012 & 0.71 & 14.67 & 6 & 2.460 & 2.54 & 60.7 & 5.5 & 2.38 & 1.676 & 3.52 & 1.42 & 6.156 & 8.757 & $5.14721 E-06$ \\
\hline 1022 & 0.64 & 14.92 & 6 & 2.515 & 2.54 & 34.0 & 5.8 & 2.13 & 1.768 & 3.52 & 1.42 & 5.998 & 8.532 & 2.95933E-06 \\
\hline 1032 & 0.81 & 14.33 & 6 & 2.387 & 2.54 & 16.1 & 6.1 & 2.01 & 1.859 & 3.52 & 1.42 & 5.969 & 8.491 & $1.40804 E-06$ \\
\hline 1042 & 0.90 & 11.33 & 6 & 1.500 & 1.52 & 3.0 & 6.4 & 1.83 & 1.951 & 3.52 & 1.42 & 5.881 & 8.365 & 4.45031 E- 07 \\
\hline 1055 & 0.67 & 15.00 & 6 & 2.548 & 2.54 & 10.7 & 6.9 & 1.58 & 2.103 & 3.52 & 1.42 & 5.783 & 8.226 & $9.65898 \mathrm{E}-07$ \\
\hline 1075 & 0.64 & 15.00 & 6 & 2.539 & 2.54 & 3.0 & 7.5 & 1.40 & 2.286 & 3.52 & 1.42 & 5.786 & 8.230 & $2.70678 \mathrm{E}-07$ \\
\hline 2002 & 0.67 & 14.33 & 6 & 2.344 & 2.54 & 47.7 & 3.8 & 2.13 & 1.158 & 3.52 & 1.42 & 5.388 & 7.665 & 4.62148E-06 \\
\hline 2013 & 0.92 & 14.50 & 6 & 2.472 & 2.54 & 3.0 & 4.4 & 1.98 & 1.341 & 3.52 & 1.42 & 5.421 & 7.711 & $2.88896 \mathrm{E}-07$ \\
\hline 2024 & 0.75 & 16.00 & 6 & 2.877 & 3.05 & 1.38 & 5.2 & 1.86 & 1.585 & 3.52 & 1.42 & 5.545 & 7.888 & $1.08199 \mathrm{E}-07$ \\
\hline 2031 & 0.50 & 9.00 & 6 & 0.668 & 0.50 & 31.1 & 5.6 & 1.62 & 1.707 & 3.52 & 1.42 & 5.427 & 7.720 & $1.51979 \mathrm{E}-05$ \\
\hline 2036 & 0.50 & 20.00 & 6 & 4.020 & 4.06 & 37.8 & 6 & 1.52 & 1.829 & 3.52 & 1.42 & 5.449 & 7.751 & $2.26573 E-06$ \\
\hline 2046 & 0.25 & 15.00 & 6 & 2.420 & 2.54 & 13.6 & 6.5 & 1.22 & 1.981 & 3.52 & 1.42 & 5.301 & 7.541 & $1.33929 \mathrm{E}-06$ \\
\hline 3007 & 0.71 & 12.33 & 6 & 1.747 & 2.04 & 23.8 & 7.6 & 2.68 & 2.316 & 3.52 & 1.42 & 7.096 & 10.095 & 2.17995E-06 \\
\hline 3013 & 0.88 & 16.58 & 6 & 3.094 & 3.05 & 11.6 & 8.2 & 2.47 & 2.499 & 3.52 & 1.42 & 7.069 & 10.056 & $7.13381 \mathrm{E}-07$ \\
\hline 3019 & 0.64 & 20.00 & 6 & 4.063 & 4.06 & 3.0 & 8.5 & 2.19 & 2.591 & 3.52 & 1.42 & 6.881 & 9.788 & 1.42397E-07 \\
\hline 3029 & 0.81 & 16.10 & 6 & 2.926 & 3.05 & 42.1 & 9.3 & 2.26 & 2.835 & 3.52 & 1.42 & 7.195 & 10.234 & $2.544 E-06$ \\
\hline 3040 & $1: 10$ & 16.10 & 8 & 2.405 & 2.54 & 34.3 & 10 & 1.77 & 3.048 & 3.52 & 1.42 & 6.918 & 9.841 & $2.58835 \mathrm{E}-06$ \\
\hline 3049 & 0.50 & 12.00 & 6 & 1.582 & 1.52 & 1.0 & 10.5 & 1.22 & 3.200 & 3.52 & 1.42 & 6.520 & 9.275 & $1.33791 \mathrm{E}-07$ \\
\hline 4023 & 0.88 & 12.75 & 6 & 1.926 & 2.04 & 5.6 & 7.5 & 2.62 & 2.286 & 3.52 & 1.42 & 7.006 & 9.966 & 5.19555E-07 \\
\hline 4033 & 0.85 & 12.50 & 6 & 1.841 & 2.04 & 9.0 & 8.0 & 2.25 & 2.438 & 3.52 & 1.42 & 6.788 & 9.656 & $8.61764 \mathrm{E}-07$ \\
\hline 4042 & 0.79 & 12.90 & 6 & 1.945 & 2.04 & 45.5 & 8.5 & 2.01 & 2.591 & 3.52 & 1.42 & 6.701 & 9.532 & 4.41365E-06 \\
\hline 4053 & 0.58 & 13.20 & 6 & 1.972 & 2.04 & 1.0 & 9.0 & 1.46 & 2.743 & 3.52 & 1.42 & 6.303 & 8.966 & $1.03122 \mathrm{E}-07$ \\
\hline & & "Measur & ed below & round sui & & & & & & & & & & \\
\hline
\end{tabular}




\section{ACT REMBCO JOINT VENTURE Ornl Wag 4 Seeps 4 \& 6 Removal Action Project}

In-Situ Hydraulic Conductivity Testing Record

August 05, 1996

\begin{tabular}{|c|c|c|c|c|c|c|}
\hline $\begin{array}{l}\text { Hole } \\
\text { No. }\end{array}$ & $\begin{array}{l}\text { Sleeve } \\
\text { No. }\end{array}$ & $\begin{array}{l}\text { Flow } \\
\text { Rate } \\
\text { (lpm) }\end{array}$ & $\begin{array}{c}\text { Gauge } \\
\text { Pressure } \\
\text { (psi) }\end{array}$ & $\begin{array}{l}\text { Effective } \\
\text { Pressure } \\
\text { (psi) }\end{array}$ & $\begin{array}{l}\text { Length of } \\
\text { Zone Tested } \\
\text { (m) }\end{array}$ & $\begin{array}{l}\text { Hydraulic Conductivity Value } \\
(\mathrm{m} / \mathrm{s})\end{array}$ \\
\hline 1001 & 1 & 20.0 & 80.0 & Negative & 0.5 & Infinity \\
\hline 1001 & 4 & 30.0 & 54.0 & Negative & 0.5 & Infinity \\
\hline 1001 & 2 & 24.0 & 44.0 & Negative & 0.5 & Infinity \\
\hline 1001 & 5 & 25.0 & 50.0 & Negative & 0.5 & Infinity \\
\hline 1001 & 6 & 30.0 & 55.0 & Negative & 0.5 & Infinity \\
\hline 1001 & 3 & 25.0 & 80.0 & Negative & 0.5 & Infinity \\
\hline 1002 & 3 & 31.0 & 54.0 & Negative & 0.5 & Infinity \\
\hline 1002 & 4 & 30.0 & 55.0 & Negative & 0.5 & Infinity \\
\hline 1002 & 6 & 27.0 & 35.0 & Negative & 0.5 & Infinity \\
\hline 1002 & 5 & 27.0 & 32.0 & Negative & 0.5 & Infinity \\
\hline 1002 & 2 & 28.0 & 52.0 & Negative & 0.5 & Infinity \\
\hline 1002 & 1 & 24.0 & 84.0 & Negative & 0.5 & Infinity \\
\hline 1003 & 1 & 25.0 & 38.0 & Negative & 0.5 & Infinity \\
\hline 1003 & 3 & 26.0 & 30.0 & Negative & 0.5 & Infinity \\
\hline 1003 & 4 & 23.0 & 28.0 & Negative & 0.5 & Infinity \\
\hline 1003 & 5 & 22.0 & 30.0 & Negative & 0.5 & Infinity \\
\hline 1003 & 6 & 23.0 & 30.0 & Negative & 0.5 & Infinity \\
\hline 1003 & 7 & 25.0 & 34.0 & Negative & 0.5 & Infinity \\
\hline 1003 & 2 & 24.0 & 30.0 & Negative & 0.5 & Infinity \\
\hline 1004 & 3 & 25.0 & 35.0 & Negative & 0.5 & Infinity \\
\hline 1004 & 7 & 26.0 & 35.0 & Negative & 0.5 & Infinity \\
\hline 1004 & 6 & 25.0 & 36.0 & Negative & 0.5 & Infinity \\
\hline 1004 & 5 & 27.0 & 40.0 & Negative & 0.5 & Infinity \\
\hline 1004 & 4 & 26.0 & 40.0 & Negative & 0.5 & Infinity \\
\hline 1004 & 2 & 26.0 & 34.0 & Negative & 0.5 & Infinity \\
\hline 1004 & 1 & 25.0 & 50.0 & Negative & 0.5 & Infinity \\
\hline 1005 & 1 & 24.0 & 36.0 & Negative & 0.5 & Infinity \\
\hline 1005 & 6 & 19.0 & 40.0 & Negative & 0.5 & Infinity \\
\hline 1005 & 5 & 20.0 & 27.0 & Negative & 0.5 & Infinity \\
\hline 1005 & 4 & 25.0 & 35.0 & Negative & 0.5 & Infinity \\
\hline 1005 & 3 & 27.0 & 38.0 & Negative & 0.5 & Infinity \\
\hline 1005 & 2 & 27.0 & 36.0 & Negative & 0.5 & Infinity \\
\hline 1006 & 3 & 24.0 & 30.0 & Negative & 0.5 & Infinity \\
\hline
\end{tabular}




\title{
ACT REMBCO JOINT VENTURE Ornl Wag 4 Seeps 4 \& 6 Removal Action Project
}

\author{
In-Situ Hydraulic Conductivity Testing Record
}

August 05, 1996

\begin{tabular}{|c|c|c|c|c|c|c|}
\hline $\begin{array}{l}\text { Hole } \\
\text { No. }\end{array}$ & $\begin{array}{l}\text { Sleeve } \\
\text { No. }\end{array}$ & $\begin{array}{l}\text { Flow } \\
\text { Rate } \\
\text { (Ipm) }\end{array}$ & \begin{tabular}{|c} 
Gauge \\
Pressure \\
(psi)
\end{tabular} & \begin{tabular}{|c|} 
Effective \\
Pressure \\
(psi)
\end{tabular} & $\begin{array}{c}\text { Length of } \\
\text { Zone Tested } \\
(\mathrm{m})\end{array}$ & $\begin{array}{l}\text { Hydraulic Conductivity Value } \\
(\mathrm{m} / \mathrm{s})\end{array}$ \\
\hline 1006 & 6 & 25.0 & 30.0 & Negative & 0.5 & Infinity \\
\hline 1006 & 5 & 25.0 & 32.0 & Negative & 0.5 & Infinity \\
\hline 1006 & 4 & 26.0 & 34.0 & Negative & 0.5 & Infinity \\
\hline 1006 & 1,2 & 24.0 & 34.0 & Negative & 1 & Infinity \\
\hline 1007 & 4 & 27.0 & 32.0 & Negative & 0.5 & Infinity \\
\hline 1007 & 1 & 24.0 & 34.0 & Negative & 0.5 & Infinity \\
\hline 1007 & 3 & 25.0 & 25.0 & Negative & 0.5 & Infinity \\
\hline 1007 & 2 & 26.0 & 33.0 & Negative & 0.5 & Infinity \\
\hline 1007 & 5 & 24.0 & 23.0 & Negative & 0.5 & Infinity \\
\hline 1007 & 7 & 15.0 & 34.0 & Negative & 0.5 & Infinity \\
\hline 1007 & 6 & 26.0 & 29.0 & Negative & 0.5 & Infinity \\
\hline 1008 & 4 & 27.0 & 27.0 & Negative & 0.5 & Infinity \\
\hline 1008 & 1,2 & 24.0 & 30.0 & Negative & 1 & Infinity \\
\hline 1008 & 8 & 25.0 & 33.0 & Negative & 0.5 & Infinity \\
\hline 1008 & 5 & 26.0 & 28.0 & Negative & 0.5 & Infinity \\
\hline 1008 & 6 & 28.0 & 32.0 & Negative & 0.5 & Infinity \\
\hline 1008 & 3 & 30.0 & 30.0 & Negative & 0.5 & Infinity \\
\hline 1008 & 7 & 25.0 & 35.0 & Negative & 0.5 & Infinity \\
\hline 1009 & 5 & 25.0 & 28.0 & Negative & 0.5 & Infinity \\
\hline 1009 & 6 & 28.0 & 34.0 & Negative & 0.5 & Infinity \\
\hline 1009 & 2 & 28.0 & 28.0 & Negative & 0.5 & Infinity \\
\hline 1009 & 3 & 24.0 & 24.0 & Negative & 0.5 & Infinity \\
\hline 1009 & 1 & 23.0 & 48.0 & Negative & 0.5 & Infinity \\
\hline 1009 & 4 & 26.0 & 27.0 & Negative & 0.5 & Infinity \\
\hline 1010 & 7 & 26.0 & 26.0 & Negative & 0.5 & Infinity \\
\hline 1010 & 5 & 32.0 & 36.0 & Negative & 0.5 & Infinity \\
\hline 1010 & 6 & 33.0 & 38.0 & Negative & 0.5 & Infinity \\
\hline 1010 & 4 & 30.0 & 35.0 & Negative & 0.5 & Infinity \\
\hline 1011 & 7 & 28.0 & 31.0 & Negative & 0.5 & Infinity \\
\hline 1011 & 4 & 31.0 & 35.0 & Negative & 0.5 & Infinity \\
\hline 1011 & 2 & 31.0 & 35.0 & Negative & 0.5 & Infinity \\
\hline 1011 & 3 & 30.0 & 35.0 & Negative & 0.5 & Infinity \\
\hline 911 & 1 & 20.0 & 50.0 & Negative & 0.5 & Infinity \\
\hline
\end{tabular}




\title{
ACT REMBCO JOINT VENTURE Ornl Wag 4 Seeps 4 \& 6 Removal Action Project
}

\author{
In-Situ Hydraulic Conductivity Testing Record
}

August 05, 1996

\begin{tabular}{|c|c|c|c|c|c|c|}
\hline $\begin{array}{l}\text { Hole } \\
\text { No. }\end{array}$ & $\begin{array}{l}\text { Sleeve } \\
\text { No. }\end{array}$ & $\begin{array}{l}\text { Flow } \\
\text { Rate } \\
\text { (lpm) }\end{array}$ & $\begin{array}{l}\text { Gauge } \\
\text { Pressure } \\
\text { (psi) }\end{array}$ & $\begin{array}{l}\text { Effective } \\
\text { Pressure } \\
\text { (psi) }\end{array}$ & $\begin{array}{l}\text { Length of } \\
\text { Zone Tested } \\
\text { (m) }\end{array}$ & $\begin{array}{l}\text { Hydraulic Conductivity Value } \\
\qquad(\mathrm{m} / \mathrm{s})\end{array}$ \\
\hline 1011 & 5 & 33.0 & 40.0 & Negative & 0.5 & Infinity \\
\hline 1011 & 6 & 33.0 & 41.0 & Negative & 0.5 & Infinity \\
\hline 1012 & 4 & 28.0 & 26.0 & Negative & 0.5 & Infinity \\
\hline 1012 & 2 & 26.0 & 28.0 & Negative & 0.5 & Infinity \\
\hline 1012 & 1 & 22.0 & 50.0 & Negative & 0.5 & Infinity \\
\hline 1012 & 3 & 28.0 & 28.0 & Negative & 0.5 & Infinity \\
\hline 1012 & 5 & 30.0 & 30.0 & Negative & 0.5 & Infinity \\
\hline 1012 & 7 & 26.0 & 32.0 & Negative & 0.5 & Infinity \\
\hline 1012 & 6 & 29.0 & 30.0 & Negative & 0.5 & Infinity \\
\hline 1013 & 3 & 29.0 & 31.0 & Negative & 0.5 & Infinity \\
\hline 1013 & 2 & 30.0 & 31.0 & Negative & 0.5 & Infinity \\
\hline 1013 & 6 & 28.0 & 31.0 & Negative & 0.5 & Infinity \\
\hline 1013 & 4 & 29.0 & 30.0 & Negative & 0.5 & Infinity \\
\hline 1013 & 5 & 28.0 & 30.0 & Negative & 0.5 & Infinity \\
\hline 1013 & 7 & 26.0 & 32.0 & Negative & 0.5 & Infinity \\
\hline 1013 & 1 & 22.0 & 45.0 & Negative & 0.5 & Infinity \\
\hline 1014 & 5 & 27.0 & 24.0 & Negative & 0.5 & Infinity \\
\hline 1014 & 4 & 30.0 & 30.0 & Negative & 0.5 & Infinity \\
\hline 1014 & 8 & 14.0 & 40.0 & Negative & 0.5 & Infinity \\
\hline 1014 & 6 & 28.0 & 30.0 & Negative & 0.5 & Infinity \\
\hline 1014 & 7 & 27.0 & 32.0 & Negative & 0.5 & Infinity \\
\hline 1014 & 3 & 31.0 & 32.0 & Negative & 0.5 & Infinity \\
\hline 1014 & 2 & 31.0 & 31.0 & Negative & 0.5 & Infinity \\
\hline 1014 & 1 & 20.0 & 45.0 & Negative & 0.5 & Infinity \\
\hline 1015 & 5 & 27.0 & 35.0 & Negative & 0.5 & Infinity \\
\hline 1015 & 7 & 25.0 & 35.0 & Negative & 0.5 & Infinity \\
\hline 1015 & 6 & 28.0 & 36.0 & Negative & 0.5 & Infinity \\
\hline 1015 & 3 & 30.0 & 34.0 & Negative & 0.5 & Infinity \\
\hline 1015 & 4 & 32.0 & 38.0 & Negative & 0.5 & Infinity \\
\hline 1015 & 2 & 25.0 & 22.0 & Negative & 0.5 & Infinity \\
\hline 1016 & 3 & 27.0 & 31.0 & Negative & 0.5 & Infinity \\
\hline 1016 & 2 & 29.0 & 34.0 & Negative & 0.5 & Infinity \\
\hline 1016 & 4 & 26.0 & 28.0 & Negative & 0.5 & Infinity \\
\hline
\end{tabular}




\section{ACT REMBCO JOINT VENTURE \\ Ornl Wag 4 Seeps 4 \& 6 Removal Action Project \\ In-Situ Hydraulic Conductivity Testing Record}

August 05, 1996

\begin{tabular}{|c|c|r|r|r|r|l|}
\hline $\begin{array}{c}\text { Hole } \\
\text { No. }\end{array}$ & $\begin{array}{c}\text { Sleeve } \\
\text { No. }\end{array}$ & $\begin{array}{r}\text { Flow } \\
\text { Rate } \\
(\mathrm{pm})\end{array}$ & $\begin{array}{c}\text { Gauge } \\
\text { Pressure } \\
\text { (psi) }\end{array}$ & $\begin{array}{c}\text { Effective } \\
\text { Pressure } \\
\text { (psi) }\end{array}$ & $\begin{array}{c}\text { Length of } \\
\text { Zone Tested } \\
\text { (m) }\end{array}$ & $\begin{array}{c}\text { Hydraulic Conductivity Value } \\
\text { (m/s) }\end{array}$ \\
\hline 1016 & 5 & 24.0 & 26.0 & Negative & 0.5 & Infinity \\
\hline 1016 & 6 & 24.0 & 33.0 & Negative & 0.5 & Infinity \\
\hline 1016 & 7 & 24.0 & 30.0 & Negative & 0.5 & Infinity \\
\hline 1026 & 1 & 22.0 & 49.0 & Negative & 0.5 & Infinity \\
\hline
\end{tabular}

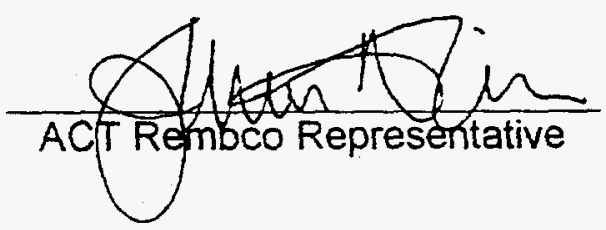




\section{ACT REMBCO JOINT VENTURE \\ Ornl Wag 4 Seeps 4 \& 6 Removal Action Project \\ In-Situ Hydraulic Conductivity Testing Record}

August 13, 1996

\begin{tabular}{|c|c|r|r|r|r|l|}
\hline $\begin{array}{c}\text { Hole } \\
\text { No. }\end{array}$ & $\begin{array}{c}\text { Sleeve } \\
\text { No. }\end{array}$ & $\begin{array}{c}\text { Flow } \\
\text { Rate } \\
(\text { lpm) }\end{array}$ & $\begin{array}{c}\text { Gauge } \\
\text { Pressure } \\
\text { (psi) }\end{array}$ & $\begin{array}{c}\text { Effective } \\
\text { Pressure } \\
\text { (psi) }\end{array}$ & $\begin{array}{c}\text { Length of } \\
\text { Zone Tested } \\
\text { (m) }\end{array}$ & $\begin{array}{c}\text { Hydraulic Conductivity Value } \\
\text { (m/s) }\end{array}$ \\
\hline 1205 & $2,3,4$ & 28.0 & 33.0 & Negative & 1.5 & Infinity \\
\hline 1205 & 1 & 20.0 & 35.0 & Negative & 0.5 & Infinity \\
\hline 1206 & 1 & 29.0 & 30.0 & Negative & 0.5 & Infinity \\
\hline 1206 & $3,4,5$ & 32.0 & 30.0 & Negative & 1.5 & Infinity \\
\hline 1210 & 1 & 29.0 & 28.0 & Negative & 0.5 & Infinity \\
\hline 1210 & $2,3,4$ & 30.0 & 30.0 & Negative & 1.5 & Infinity \\
\hline 1211 & $4,5,6$ & 33.0 & 30.0 & Negative & 1.5 & Infinity \\
\hline 1211 & 1 & 30.0 & 30.0 & Negative & 0.5 & Infinity \\
\hline 1212 & 1 & 30.0 & 29.0 & Negative & 0.5 & Infinity \\
\hline 1212 & $4,5,6$ & 32.0 & 30.0 & Negative & 1.5 & Infinity \\
\hline 1213 & $2,3,4$ & 34.0 & 29.0 & Negative & 1.5 & Infinity \\
\hline 1213 & 1 & 19.0 & 30.0 & Negative & 0.5 & Infinity \\
\hline 1214 & $2,3,4$ & 28.0 & 32.0 & Negative & 1.5 & Infinity \\
\hline 1214 & 1 & 23.0 & 35.0 & Negative & 0.5 & Infinity \\
\hline
\end{tabular}

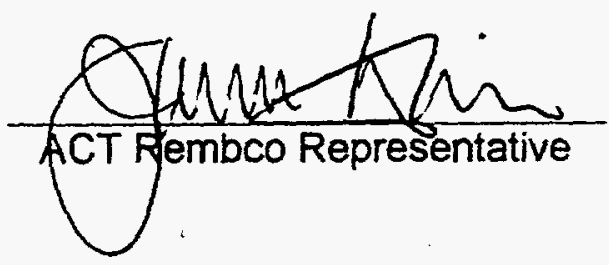




\section{ACT REMBCO JOINT VENTURE Ornl Wag 4 Seeps 4 \& 6 Removal Action Project}

In-Situ Hydraulic Conductivity Testing Record

August 15, 1996

\begin{tabular}{|c|c|c|c|c|c|l|}
\hline $\begin{array}{c}\text { Hole } \\
\text { No. }\end{array}$ & $\begin{array}{c}\text { Sleeve } \\
\text { No. }\end{array}$ & $\begin{array}{c}\text { Flow } \\
\text { Rate } \\
\text { (ipm) }\end{array}$ & $\begin{array}{c}\text { Gauge } \\
\text { Pressure } \\
\text { (psi) }\end{array}$ & $\begin{array}{c}\text { Effective } \\
\text { Pressure } \\
\text { (psi) }\end{array}$ & $\begin{array}{c}\text { Length of } \\
\text { Zone Tested } \\
\text { (m) }\end{array}$ & $\begin{array}{l}\text { Hydraulic Conductivity Value } \\
\text { (m/s) }\end{array}$ \\
\hline 1017 & 1 & 24.0 & 23.0 & $(-)$ & 0.5 & Infinity \\
\hline 1017 & 4 & 26.0 & 32.0 & $(-)$ & 0.5 & Infinity \\
\hline 1017 & 2 & 30.0 & 28.0 & $(-)$ & 0.5 & Infinity \\
\hline 1017 & 6 & 28.0 & 30.0 & $(-)$ & 0.5 & Infinity \\
\hline 1017 & 5 & 29.0 & 29.0 & $(-)$ & 0.5 & Infinity \\
\hline 1017 & 7 & 28.0 & 30.0 & $(-)$ & 0.5 & Infinity \\
\hline 1017 & 3 & 24.0 & 37.0 & $(-)$ & 0.5 & Infinity \\
\hline 1018 & 7 & 26.0 & 32.0 & $(-)$ & 0.5 & Infinity \\
\hline 1018 & 5 & 29.0 & 31.0 & $(-)$ & 0.5 & Infinity \\
\hline 1018 & 2 & 26.0 & 36.0 & $(-)$ & 0.5 & Infinity \\
\hline 1018 & 6 & 28.0 & 38.0 & $(-)$ & 0.5 & Infinity \\
\hline 1018 & 1 & 26.0 & 35.0 & $(-)$ & 0.5 & Infinity \\
\hline 1018 & 3 & 28.0 & 31.0 & $(-)$ & 0.5 & Infinity \\
\hline 1018 & 4 & 29.0 & 32.0 & $(-)$ & 0.5 & Infinity \\
\hline 1019 & 4 & 29.0 & 30.0 & $(-)$ & 0.5 & Infinity \\
\hline 1019 & 3 & 30.0 & 28.0 & $(-)$ & 0.5 & Infinity \\
\hline 1019 & 6 & 27.0 & 32.0 & $(-)$ & 0.5 & Infinity \\
\hline 1019 & 5 & 29.0 & 32.0 & $(-)$ & 0.5 & Infinity \\
\hline 1019 & 7 & 27.0 & 33.0 & $(-)$ & 0.5 & Infinity \\
\hline 1019 & 2 & 29.0 & 29.0 & $(-)$ & 0.5 & Infinity \\
\hline 1019 & 1 & 26.0 & 30.0 & $(-)$ & 0.5 & Infinity \\
\hline 1020 & 4 & 25.0 & 38.0 & $(-)$ & 0.5 & Infinity \\
\hline 1020 & 2 & 24.0 & 38.0 & $(-)$ & 0.5 & Infinity \\
\hline 1020 & 3 & 12.5 & 26.0 & $(-)$ & 0.5 & Infinity \\
\hline 1020 & 5 & 28.0 & 42.0 & $(-)$ & 0.5 & Infinity \\
\hline 1020 & 6 & 26.0 & 45.0 & $(-)$ & 0.5 & Infinity \\
\hline 1020 & 1 & 25.0 & 32.0 & $(-)$ & 0.5 & Infinity \\
\hline 1021 & 1 & 24.0 & 38.0 & $(-)$ & 0.5 & Infinity \\
\hline 1022 & 1 & 28.0 & 29.0 & $(-)$ & 0.5 & Infinity \\
\hline 1023 & 1 & 26.0 & 35.0 & $(-)$ & 0.5 & Infinity \\
\hline 1024 & 1 & 29.0 & 29.0 & $(-)$ & 0.5 & Infinity \\
\hline 1025 & 1 & 26.0 & 34.0 & $(-)$ & 0.5 & Infinity \\
\hline 026 & 1 & 27.0 & 34.0 & $(-)$ & 0.5 & Infinity \\
\hline & & & & & & \\
\hline
\end{tabular}




\section{ACT REMBCO JOINT VENTURE \\ Ornl Wag 4 Seeps 4 \& 6 Removal Action Project \\ In-Situ Hydraulic Conductivity Testing Record}

August 15, 1996

\begin{tabular}{|c|c|c|c|c|c|l|}
\hline $\begin{array}{c}\text { Hole } \\
\text { No. }\end{array}$ & $\begin{array}{c}\text { Sleeve } \\
\text { No. }\end{array}$ & $\begin{array}{c}\text { Flow } \\
\text { Rate } \\
(\mathrm{pm})\end{array}$ & $\begin{array}{c}\text { Gauge } \\
\text { Pressure } \\
(\mathrm{psi})\end{array}$ & $\begin{array}{c}\text { Effective } \\
\text { Pressure } \\
(\mathrm{psi})\end{array}$ & $\begin{array}{c}\text { Length of } \\
\begin{array}{c}\text { Zone Tested } \\
(\mathrm{m})\end{array}\end{array}$ & $\begin{array}{c}\text { Hydraulic Conductivity Value } \\
\text { (m/s) }\end{array}$ \\
\hline 1027 & 1 & 30.0 & 29.0 & $(-)$ & 0.5 & Infinity \\
\hline 1028 & 1 & 29.0 & 50.0 & $(-)$ & 0.5 & Infinity \\
\hline 1030 & 1 & 30.0 & 30.0 & $(-)$ & 0.5 & Infinity \\
\hline 1031 & 1 & 28.0 & 34.0 & $(-)$ & 0.5 & Infinity \\
\hline 1032 & 1 & 29.0 & 30.0 & $(-)$ & 0.5 & Infinity \\
\hline 1033 & 1 & 27.0 & 33.0 & $(-)$ & 0.5 & Infinity \\
\hline 1035 & 1 & 27.0 & 49.0 & $(-)$ & 0.5 & Infinity \\
\hline 1036 & 1 & 29.0 & 30.0 & $(-)$ & 0.5 & Infinity \\
\hline 1037 & 1 & 27.0 & 32.0 & $(-)$ & 0.5 & Infinity \\
\hline 1038 & 1 & 28.0 & 30.0 & $(-)$ & 0.5 & Infinity \\
\hline 1039 & 1 & 27.0 & 29.0 & $(-)$ & 0.5 & Infinity \\
\hline
\end{tabular}

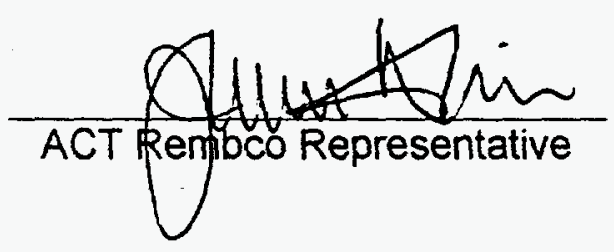




\section{ACT REMBCO JOINT VENTURE Ornl Wag 4 Seeps 4 \& 6 Removal Action Project}

In-Situ Hydraulic Conductivity Testing Record

August 16, 1996

\begin{tabular}{|c|c|c|c|c|c|c|}
\hline $\begin{array}{l}\text { Hole } \\
\text { No. }\end{array}$ & $\begin{array}{l}\text { Sleeve } \\
\text { No. }\end{array}$ & $\begin{array}{l}\text { Flow } \\
\text { Rate } \\
\text { (lpm) }\end{array}$ & $\begin{array}{c}\text { Gauge } \\
\text { Pressure } \\
\text { (psi) }\end{array}$ & $\begin{array}{c}\text { Effective } \\
\text { Pressure } \\
\text { (psi) }\end{array}$ & $\begin{array}{l}\text { Length of } \\
\text { Zone Tested } \\
\text { (m) }\end{array}$ & $\begin{array}{l}\text { Hydraulic Conductivity Value } \\
\qquad(\mathrm{m} / \mathrm{s})\end{array}$ \\
\hline 1021 & 2 & 25.0 & 68.0 & $(-)$ & 0.5 & Infinity \\
\hline 1021 & 5 & 30.0 & 42.0 & $(-)$ & 0.5 & Infinity \\
\hline 1021 & 8 & 31.0 & 48.0 & $(-)$ & 0.5 & Infinity \\
\hline 1021 & 7 & 32.0 & 45.0 & $(-)$ & 0.5 & Infinity \\
\hline 1021 & 6 & 28.0 & 30.0 & $(-)$ & 0.5 & \begin{tabular}{|l} 
Infinity \\
\end{tabular} \\
\hline 1021 & 4 & 30.0 & 41.0 & $(-)$ & 0.5 & Infinity \\
\hline 1021 & 3 & 30.0 & 40.0 & $(-)$ & 0.5 & Infinity \\
\hline 1022 & 4 & 26.0 & 40.0 & $(-)$ & 0.5 & Infinity \\
\hline 1022 & 2 & 25.0 & 35.0 & $(-)$ & 0.5 & Infinity \\
\hline 1022 & 8 & 26.0 & 37.0 & $(-)$ & 0.5 & Infinity \\
\hline 1022 & 3 & 27.0 & 38.0 & $(-)$ & 0.5 & Infinity \\
\hline 1022 & 7 & 28.0 & 40.0 & $(-)$ & 0.5 & Infinity \\
\hline 1022 & 6 & 28.0 & 40.0 & $(-)$ & 0.5 & Infinity \\
\hline 1022 & 5 & 28.0 & 42.0 & $(-)$ & 0.5 & Infinity \\
\hline 1023 & 4 & 26.0 & 28.0 & $(-)$ & 0.5 & Infinity \\
\hline 1023 & 6 & 24.0 & 30.0 & $(-)$ & 0.5 & Infinity \\
\hline 1023 & 5 & 25.0 & 28.0 & $(-)$ & 0.5 & Infinity \\
\hline 1023 & 7 & 24.0 & 30.0 & $(-)$ & 0.5 & Infinity \\
\hline 1023 & 2 & 26.0 & 37.0 & $(-)$ & 0.5 & Infinity \\
\hline 1023 & 3 & 32.0 & 28.0 & $(-)$ & 0.5 & Infinity \\
\hline 1024 & 5 & 29.0 & 29.0 & $(-)$ & 0.5 & Infinity \\
\hline 1024 & 3 & 29.0 & 29.0 & $(-)$ & 0.5 & Infinity \\
\hline 1024 & 6 & 28.0 & 28.0 & $(-)$ & 0.5 & Infinity \\
\hline 1024 & 7 & 27.0 & 27.0 & $(-1)$ & 0.5 & Infinity \\
\hline 1024 & 2 & 30.0 & 30.0 & $(-)$ & 0.5 & Infinity \\
\hline 1024 & 8 & 28.0 & 28.0 & $(-)$ & 0.5 & Infinity \\
\hline 1024 & 4 & 30.0 & 27.0 & $(-)$ & 0.5 & Infinity \\
\hline 1027 & 5 & 26.0 & 47.0 & $(-)$ & 0.5 & Infinity \\
\hline 1027 & 4 & 28.0 & 42.0 & $(-)$ & 0.5 & Infinity \\
\hline 1027 & 7 & 30.0 & 65.0 & $(-)$ & 0.5 & Infinity \\
\hline 1027 & 6 & 25.0 & 51.0 & $(-)$ & 0.5 & Infinity \\
\hline 1027 & 2 & 25.0 & 46.0 & $(-)$ & 0.5 & Infinity \\
\hline 927 & 3 & 25.0 & 38.0 & $(-)$ & 0.5 & Infinity \\
\hline
\end{tabular}




\section{ACT REMBCO JOINT VENTURE Ornl Wag 4 Seeps 4 \& 6 Removal Action Project In-Situ Hydraulic Conductivity Testing Record}

August 16, 1996

\begin{tabular}{|c|c|c|c|c|c|l|}
\hline $\begin{array}{c}\text { Hole } \\
\text { No. }\end{array}$ & $\begin{array}{c}\text { Sleeve } \\
\text { No. }\end{array}$ & $\begin{array}{c}\text { Flow } \\
\text { Rate } \\
(\text { (pm) }\end{array}$ & $\begin{array}{c}\text { Gauge } \\
\text { Pressure } \\
\text { (psi) }\end{array}$ & $\begin{array}{c}\text { Effective } \\
\text { Pressure } \\
\text { (psi) }\end{array}$ & $\begin{array}{c}\text { Length of } \\
\text { Zone Tested } \\
\text { (m) }\end{array}$ & $\begin{array}{c}\text { Hydraulic Conductivity Value } \\
\text { (m/s) }\end{array}$ \\
\hline 1030 & 5 & 30.0 & 40.0 & $(-)$ & 0.5 & Infinity \\
\hline 1030 & 6 & 28.0 & 40.0 & $(-)$ & 0.5 & Infinity \\
\hline 1030 & 4 & 27.0 & 35.0 & $(-)$ & 0.5 & Infinity \\
\hline 1030 & 2 & 25.0 & 30.0 & $(-)$ & 0.5 & Infinity \\
\hline 1030 & 7 & 35.0 & 50.0 & $(-)$ & 0.5 & Infinity \\
\hline 1030 & 3 & 26.0 & 35.0 & $(-)$ & 0.5 & Infinity \\
\hline 1030 & 8 & 35.0 & 50.0 & $(-)$ & 0.5 & Infinity \\
\hline 1032 & 3 & 25.0 & 52.0 & $(-)$ & 0.5 & Infinity \\
\hline 1032 & 8 & 27.0 & 54.0 & $(-)$ & 0.5 & Infinity \\
\hline 1032 & 4 & 27.0 & 46.0 & $(-)$ & 0.5 & Infinity \\
\hline 1032 & 5 & 24.0 & 48.0 & $(-)$ & 0.5 & Infinity \\
\hline 1032 & 6 & 25.0 & 50.0 & $(-)$ & 0.5 & Infinity \\
\hline 1032 & 2 & 29.0 & 52.0 & $(-)$ & 0.5 & Infinity \\
\hline 1032 & 7 & 25.0 & 50.0 & $(-)$ & 0.5 & Infinity \\
\hline 1037 & 7 & 25.0 & 53.0 & $(-)$ & 0.5 & Infinity \\
\hline 1037 & 5 & 29.0 & 40.0 & $(-)$ & 0.5 & Infinity \\
\hline 1037 & 6 & 27.0 & 46.0 & $(-)$ & 0.5 & Infinity \\
\hline 1037 & 2 & 28.0 & 37.0 & $(-)$ & 0.5 & Infinity \\
\hline 1037 & 4 & 28.0 & 38.0 & $(-)$ & 0.5 & Infinity \\
\hline 1037 & 3 & 27.0 & 36.0 & $(-)$ & 0.5 & Infinity \\
\hline 1040 & 6 & 27.0 & 70.0 & $(-)$ & 0.5 & Infinity \\
\hline 1040 & 4 & 27.0 & 54.0 & $(-)$ & 0.5 & Infinity \\
\hline 1040 & 2 & 26.0 & 49.0 & $(-)$ & 0.5 & Infinity \\
\hline 1040 & 1 & 20.0 & 68.0 & $(-)$ & 0.5 & Infinity \\
\hline 1040 & 3 & 27.0 & 53.0 & $(-)$ & 0.5 & Infinity \\
\hline 1040 & 5 & 28.0 & 58.0 & $(-)$ & 0.5 & Infinity \\
\hline 1041 & 1 & 23.0 & 70.0 & $(-)$ & 0.5 & Infinity \\
\hline 1042 & 3 & 27.0 & 62.0 & $(-)$ & 0.5 & Infinity \\
\hline 1042 & 6 & 29.0 & 73.0 & $(-)$ & 0.5 & Infinity \\
\hline 1042 & 5 & 28.0 & 70.0 & $(-)$ & 0.5 & Infinity \\
\hline 1042 & 4 & 27.0 & 62.0 & $(-)$ & 0.5 & Infinity \\
\hline 1042 & 1 & 20.0 & 68.0 & $(-)$ & 0.5 & Infinity \\
\hline 1042 & 2 & 26.0 & 60.0 & $(-)$ & 0.5 & Infinity \\
\hline & & & & & & \\
\hline
\end{tabular}




\title{
ACT REMBCO JOINT VENTURE Ornl Wag 4 Seeps 4 \& 6 Removal Action Project
}

In-Situ Hydraulic Conductivity Testing Record

\author{
August 16, 1996
}

\begin{tabular}{|c|c|c|c|c|c|c|}
\hline $\begin{array}{l}\text { Hole } \\
\text { No. }\end{array}$ & $\begin{array}{l}\text { Sleeve } \\
\text { No. }\end{array}$ & $\begin{array}{l}\text { Flow } \\
\text { Rate } \\
\text { (lpm) }\end{array}$ & $\begin{array}{c}\text { Gauge } \\
\text { Pressure } \\
\text { (psi) }\end{array}$ & $\begin{array}{c}\text { Effective } \\
\text { Pressure } \\
\text { (psi) }\end{array}$ & $\begin{array}{l}\text { Length of } \\
\text { Zone Tested } \\
\text { (m) }\end{array}$ & $\begin{array}{l}\text { Hydraulic Conductivity Value } \\
\qquad(\mathrm{m} / \mathrm{s})\end{array}$ \\
\hline 1043 & 1 & 27.0 & 71.0 & $(-)$ & 0.5 & Infinity \\
\hline 1044 & 3 & 27.0 & 80.0 & $(-)$ & 0.5 & Infinity \\
\hline 1044 & 2 & 25.0 & 55.0 & $(-)$ & 0.5 & Infinity \\
\hline 1044 & 4 & 29.0 & 80.0 & $(-)$ & 0.5 & Infinity \\
\hline 1044 & 5 & 27.0 & 90.0 & 10 & 0.5 & $1.02 E-04$ \\
\hline 1044 & 7 & 24.0 & 93.0 & 15 & 0.5 & $6.07 E-05$ \\
\hline 1044 & 8 & 26.0 & 80.0 & $(-1)$ & 0.5 & Infinity \\
\hline 1044 & 6 & 26.0 & 93.0 & 13 & 0.5 & Infinity \\
\hline 1046 & 1 & 25.0 & 68.0 & $(-)$ & 0.5 & Infinity \\
\hline 1047 & 4 & 28.0 & 66.0 & $(-)$ & 0.5 & Infinity \\
\hline 1047 & 5 & 28.0 & 70.0 & $(-)$ & 0.5 & Infinity \\
\hline 1047 & 6 & 28.0 & 83.0 & 3 & 0.5 & $3.54 \mathrm{E}-04$ \\
\hline 1047 & 3 & 29.0 & 61.0 & $(-)$ & 0.5 & Infinity \\
\hline 1047 & 2 & 27.0 & 57.0 & $(-)$ & 0.5 & Infinity \\
\hline 1050 & 6 & 28.0 & 63.0 & $(-)$ & 0.5 & Infinity \\
\hline 1050 & 7 & 28.0 & 68.0 & $(-)$ & 0.5 & Infinity \\
\hline 1050 & 3 & 27.0 & 65.0 & $(-1)$ & 0.5 & Infinity \\
\hline 1050 & 8 & 26.0 & 71.0 & $(-)$ & 0.5 & Infinity \\
\hline 1050 & 9 & 20.0 & 75.0 & $(-)$ & 0.5 & Infinity \\
\hline 1050 & 5 & 23.0 & 60.0 & $(-)$ & 0.5 & Infinity \\
\hline 1050 & $\overline{4}$ & 29.0 & 65.0 & $(-)$ & 0.5 & Infinity \\
\hline 1050 & 2 & 28.0 & 64.0 & $(-)$ & 0.5 & Infinity \\
\hline 1053 & 4 & 28.0 & 54.0 & $(-)$ & 0.5 & Infinity \\
\hline 1053 & 5 & 26.0 & 58.0 & $(-)$ & 0.5 & Infinity \\
\hline 1053 & 6 & 32.0 & 69.0 & $(-)$ & 0.5 & Infinity \\
\hline 1053 & 2 & 25.0 & 55.0 & $(-)$ & 0.5 & Infinity \\
\hline 1053 & 7 & 25.0 & 64.0 & $(-)$ & 0.5 & Infinity \\
\hline 1053 & 8 & 23.0 & 74.0 & $(-)$ & 0.5 & Infinity \\
\hline 1053 & 3 & 31.0 & 56.0 & $(-)$ & 0.5 & Infinity \\
\hline 1055 & 2,3 & 20.0 & 56.0 & $(-)$ & 1 & Infinity \\
\hline 1055 & 4,5 & 19.0 & 56.0 & $(-)$ & 1 & Infinity \\
\hline 1055 & 6,7 & 25.0 & 55.0 & $(-)$ & 1 & Infinity \\
\hline 057 & 1 & 22.0 & 69.0 & $(-)$ & 0.5 & Infinity \\
\hline
\end{tabular}




\section{ACT REMBCO JOINT VENTURE Ornl Wag 4 Seeps 4 \& 6 Removal Action Project \\ In-Situ Hydraulic Conductivity Testing Record}

August 16, 1996

\begin{tabular}{|c|c|c|c|c|c|c|}
\hline $\begin{array}{l}\text { Hole } \\
\text { No. }\end{array}$ & $\begin{array}{l}\text { Sleeve } \\
\text { No. }\end{array}$ & $\begin{array}{l}\text { Flow } \\
\text { Rate } \\
\text { (lpm) }\end{array}$ & \begin{tabular}{|c|} 
Gauge \\
Pressure \\
(psi)
\end{tabular} & \begin{tabular}{|c} 
Effective \\
Pressure \\
(psi)
\end{tabular} & $\begin{array}{l}\text { Length of } \\
\text { Zone Tested } \\
(\mathrm{m})\end{array}$ & $\begin{array}{l}\text { Hydraulic Conductivity Value } \\
\qquad(\mathrm{m} / \mathrm{s})\end{array}$ \\
\hline 1057 & 2,3 & 20.0 & 56.0 & $(-)$ & 1 & Infinity \\
\hline 1057 & 4,5 & 20.0 & 55.0 & $(-)$ & 1 & Infinity \\
\hline 1057 & 6,7 & 25.0 & 56.0 & $(-)$ & 1 & Infinity \\
\hline 1210 & 7 & 30.0 & 45.0 & $(-)$ & 0.5 & Infinity \\
\hline 1210 & 6 & 22.0 & 142.0 & 72 & 0.5 & $1.16 \mathrm{E}-05$ \\
\hline 1210 & 5 & 6.5 & 175.0 & 157 & 0.5 & $1.57 \mathrm{E}-06$ \\
\hline 1211 & 5 & 11.0 & 165.0 & 125 & 0.5 & 3.34E-06 \\
\hline 1211 & 6 & 9.0 & 152.0 & 117 & 0.5 & $2.92 E-06$ \\
\hline 1211 & 4 & 25.0 & 35.0 & $(-)$ & 0.5 & Infinity \\
\hline 1212 & 6 & 27.0 & 30.0 & $(-)$ & 0.5 & Infinity \\
\hline 1212 & 5 & 27.0 & 35.0 & $(-)$ & 0.5 & Infinity \\
\hline 1212 & 4 & 30.0 & 30.0 & $(-)$ & 0.5 & Infinity \\
\hline 1213 & 5 & 29.0 & 29.0 & $(-)$ & 0.5 & \begin{tabular}{|l|} 
Infinity \\
\end{tabular} \\
\hline 1213 & 4 & 27.0 & 50.0 & $(-)$ & 0.5 & Infinity \\
\hline 1213 & 3 & 28.0 & 85.0 & $(-)$ & 0.5 & Infinity \\
\hline
\end{tabular}




\section{ACT REMBCO JOINT VENTURE Ornl Wag 4 Seeps 4 \& 6 Removal Action Project}

In-Situ Hydraulic Conductivity Testing Record

August 19, 1996

\begin{tabular}{|c|c|c|c|c|c|c|}
\hline $\begin{array}{l}\text { Hole } \\
\text { No. }\end{array}$ & $\begin{array}{l}\text { Sleeve } \\
\text { No. }\end{array}$ & $\begin{array}{l}\text { Flow } \\
\text { Rate } \\
\text { (lpm) }\end{array}$ & $\begin{array}{c}\text { Gauge } \\
\text { Pressure } \\
\text { (psi) }\end{array}$ & $\begin{array}{c}\text { Effective } \\
\text { Pressure } \\
\text { (psi) }\end{array}$ & $\begin{array}{c}\text { Length of } \\
\text { Zone Tested } \\
\text { (m) }\end{array}$ & $\begin{array}{l}\text { Hydraulic Conductivity Value } \\
\qquad(\mathrm{m} / \mathrm{s})\end{array}$ \\
\hline 1062 & 1 & 27.0 & 90.0 & 10 & 0.5 & $1.02 E-04$ \\
\hline 1063 & 2,3 & 26.0 & 20.0 & $(-)$ & 1 & Infinity \\
\hline 1063 & 6,7 & 23.0 & 20.0 & $(-1)$ & 1 & Infinity \\
\hline 1063 & 4,5 & 23.0 & 23.0 & $(-)$ & 1 & Infinity \\
\hline 1063 & 1 & 28.0 & 26.0 & $(-)$ & 1 & Infinity \\
\hline 1065 & 4,5 & 23.0 & 27.0 & $(-)$ & 1 & Infinity \\
\hline 1065 & 6,7 & 22.0 & 28.0 & $(-)$ & 1 & Infinity \\
\hline 1065 & 2,3 & 21.0 & 28.0 & $(-)$ & 1 & Infinity \\
\hline 1067 & 4,5 & 23.0 & 27.0 & $(-)$ & 0.5 & Infinity \\
\hline 1067 & 6,7 & 22.0 & 28.0 & $(-)$ & 0.5 & Infinity \\
\hline 1067 & 2,3 & 27.0 & 23.0 & $(-)$ & 0.5 & Infinity \\
\hline 1070 & 2,3 & 27.0 & 24.0 & $(-1)$ & 0.5 & Infinity \\
\hline 1070 & 4,5 & 28.0 & 30.0 & $(-)$ & 0.5 & Infinity \\
\hline 1070 & 6,7 & 22.0 & 29.0 & $(-1)$ & 0.5 & Infinity \\
\hline 1070 & 8,9 & 26.0 & 35.0 & $(-)$ & 0.5 & Infinity \\
\hline
\end{tabular}




\section{ACT REMBCO JOINT VENTURE \\ Ornl Wag 4 Seeps 4 \& 6 Removal Action Project \\ In-Situ Hydraulic Conductivity Testing Record}

August 19, 1996

\begin{tabular}{|c|c|c|c|c|c|l|}
\hline $\begin{array}{c}\text { Hole } \\
\text { No. }\end{array}$ & $\begin{array}{c}\text { Sleeve } \\
\text { No. }\end{array}$ & $\begin{array}{c}\text { Flow } \\
\text { Rate } \\
\text { (pm) }\end{array}$ & $\begin{array}{c}\text { Gauge } \\
\text { Pressure } \\
\text { (psi) }\end{array}$ & $\begin{array}{c}\text { Effective } \\
\text { Pressure } \\
\text { (psi) }\end{array}$ & $\begin{array}{c}\text { Length of } \\
\text { Zone Tested } \\
(\mathrm{m})\end{array}$ & $\begin{array}{l}\text { Hydraulic Conductivity Value } \\
(\mathrm{m} / \mathrm{s})\end{array}$ \\
\hline 1047 & 1 & 22.0 & 17.0 & $(-)$ & 0.5 & Infinity \\
\hline 1048 & 1 & 21.0 & 19.0 & $(-)$ & 0.5 & Infinity \\
\hline 1049 & 1 & 22.0 & 35.0 & $(-)$ & 0.5 & Infinity \\
\hline 1050 & 1 & 27.0 & 27.0 & $(-)$ & 0.5 & Infinity \\
\hline 1052 & 1 & 24.0 & 21.0 & $(-)$ & 0.5 & Infinity \\
\hline 1053 & 1 & 25.0 & 20.0 & $(-)$ & 0.5 & Infinity \\
\hline 1054 & 1 & 23.0 & 23.0 & $(-)$ & 0.5 & Infinity \\
\hline 1055 & 1 & 26.0 & 24.0 & $(-)$ & 0.5 & Infinity \\
\hline 1056 & 1 & 27.0 & 24.0 & $(-)$ & 0.5 & Infinity \\
\hline 1057 & 1 & 27.0 & 23.0 & $(-)$ & 0.5 & Infinity \\
\hline 1059 & 1 & 27.0 & 24.0 & $(-)$ & 0.5 & Infinity \\
\hline 1060 & 2,3 & 26.0 & 17.0 & $(-)$ & 1 & Infinity \\
\hline 1060 & 6,7 & 25.0 & 30.0 & $(-)$ & 1 & Infinity \\
\hline 1060 & 1 & 26.0 & 23.0 & $(-)$ & 0.5 & Infinity \\
\hline 1060 & 4,5 & 22.0 & 24.0 & $(-)$ & 1 & Infinity \\
\hline 1061 & 1 & 27.0 & 24.0 & $(-)$ & 0.5 & Infinity \\
\hline
\end{tabular}




\section{ACT REMBCO JOINT VENTURE \\ Ornl Wag 4 Seeps 4 \& 6 Removal Action Project \\ In-Situ Hydraulic Conductivity Testing Record}

September 09, 1996

\begin{tabular}{|c|c|c|c|c|c|l|}
\hline $\begin{array}{c}\text { Hole } \\
\text { No. }\end{array}$ & $\begin{array}{c}\text { Sleeve } \\
\text { No. }\end{array}$ & $\begin{array}{c}\text { Flow } \\
\text { Rate } \\
(\mathrm{Ipm})\end{array}$ & $\begin{array}{c}\text { Gauge } \\
\text { Pressure } \\
(\mathrm{psi})\end{array}$ & $\begin{array}{c}\text { Effective } \\
\text { Pressure } \\
\text { (psi) }\end{array}$ & $\begin{array}{c}\text { Length of } \\
\begin{array}{c}\text { Zone Tested } \\
(\mathrm{m})\end{array}\end{array}$ & $\begin{array}{c}\text { Hydraulic Conductivity Value } \\
\text { (m/s) }\end{array}$ \\
\hline 1075 & 1 & 4.0 & 90.0 & 75 & 0.5 & $2.02 \mathrm{E}-06$ \\
\hline 1080 & 2,3 & 24.0 & 25.0 & $(-)$ & 0.5 & Infinity \\
\hline 1083 & 1 & 24.0 & 20.0 & $(-)$ & 0.5 & Infinity \\
\hline 1080 & 4,5 & 24.0 & 30.0 & $(-)$ & 0.5 & Infinity \\
\hline 1080 & 1 & 22.0 & 26.0 & $(-)$ & 0.5 & Infinity \\
\hline 1083 & 2,3 & 26.0 & 20.0 & $(-)$ & 0.5 & Infinity \\
\hline 1083 & 4,5 & 21.0 & 16.0 & $(-)$ & 0.5 & Infinity \\
\hline 1083 & 6,7 & 17.0 & 23.0 & $(-)$ & 0.5 & Infinity \\
\hline 1075 & 2,3 & 20.0 & 22.0 & $(-)$ & 0.5 & Infinity \\
\hline 1075 & 4,5 & 22.0 & 21.0 & $(-)$ & 0.5 & Infinity \\
\hline 1075 & 6,7 & 21.0 & 21.0 & $(-)$ & 0.5 & Infinity \\
\hline & & & & & \\
\end{tabular}




\title{
ACT REMBCO JOINT VENTURE Ornl Wag 4 Seeps 4 \& 6 Removal Action Project
}

\author{
In-Situ Hydraulic Conductivity Testing Record
}

September 13, 1996

\begin{tabular}{|c|c|c|c|c|c|l|}
\hline $\begin{array}{c}\text { Hole } \\
\text { No. }\end{array}$ & $\begin{array}{c}\text { Sleeve } \\
\text { No. }\end{array}$ & $\begin{array}{c}\text { Flow } \\
\text { Rate } \\
\text { (lpm) }\end{array}$ & $\begin{array}{c}\text { Gauge } \\
\text { Pressure } \\
\text { (psi) }\end{array}$ & $\begin{array}{c}\text { Effective } \\
\text { Pressure } \\
\text { (psi) }\end{array}$ & $\begin{array}{c}\text { Length of } \\
\text { Zone Tested } \\
(\mathrm{m})\end{array}$ & $\begin{array}{l}\text { Hydraulic Conductivity Value } \\
\text { (m/s) }\end{array}$ \\
\hline 2001 & 1 & 17.0 & 68.0 & $(-)$ & 0.5 & Infinity \\
\hline 2001 & 6 & 16.0 & 25.0 & $(-)$ & 0.5 & Infinity \\
\hline 2002 & 4 & 20.0 & 19.0 & $(-)$ & 0.5 & Infinity \\
\hline 2002 & 5 & 18.0 & 21.0 & $(-)$ & 0.5 & Infinity \\
\hline 2002 & 2 & 5.5 & 88.0 & 75 & 0.5 & \\
\hline 2002 & 3 & 28.0 & 47.0 & $(-)$ & 0.5 & Infinity \\
\hline 2006 & 4 & 18.0 & 23.0 & $(-)$ & 0.5 & Infinity \\
\hline 2006 & 5 & 19.0 & 19.0 & $(-)$ & 0.5 & Infinity \\
\hline 2006 & 1 & 33.0 & 42.0 & $(-)$ & 0.5 & Infinity \\
\hline 2006 & 2 & 18.0 & 21.0 & $(-)$ & 0.5 & Infinity \\
\hline 2006 & 3 & 10.0 & 36.0 & $(-)$ & 0.5 & Infinity \\
\hline 2013 & 4 & 20.0 & 19.0 & $(-)$ & 0.5 & Infinity \\
\hline 2013 & 3 & 16.0 & 25.0 & $(-)$ & 0.5 & Infinity \\
\hline 2013 & 2 & 23.0 & 15.0 & $(-)$ & 0.5 & Infinity \\
\hline 2013 & 1 & 23.0 & 17.0 & $(-)$ & 0.5 & Infinity \\
\hline 2013 & 5 & 18.0 & 23.0 & $(-)$ & 0.5 & Infinity \\
\hline 2018 & 4 & 22.0 & 16.0 & $(-)$ & 0.5 & Infinity \\
\hline 2018 & 3 & 23.0 & 17.0 & $(-)$ & 0.5 & Infinity \\
\hline 2018 & 5 & 24.0 & 15.0 & $(-)$ & 0.5 & Infinity \\
\hline 2018 & 2 & 23.0 & 16.0 & $(-)$ & 0.5 & Infinity \\
\hline 2018 & 6 & 23.0 & 16.0 & $(-)$ & 0.5 & Infinity \\
\hline 2018 & 1 & 23.0 & 18.0 & $(-)$ & 0.5 & Infinity \\
\hline 2022 & 2 & 22.0 & 18.0 & $(-)$ & 0.5 & Infinity \\
\hline 2022 & 5 & 23.0 & 18.0 & $(-)$ & 0.5 & Infinity \\
\hline 2022 & 6 & 20.0 & 22.0 & $(-)$ & 0.5 & Infinity \\
\hline 2022 & 3 & 22.0 & 19.0 & $(-)$ & 0.5 & Infinity \\
\hline 2022 & 4 & 20.0 & 20.0 & $(-)$ & 0.5 & Infinity \\
\hline 2022 & 1 & 22.0 & 16.0 & $(-)$ & 0.5 & Infinity \\
\hline 2024 & 3 & 24.0 & 15.0 & $(-)$ & 0.5 & Infinity \\
\hline 2024 & 1 & 22.0 & 17.0 & $(-)$ & 0.5 & Infinity \\
\hline 2024 & 2 & 21.0 & 21.0 & $(-)$ & 0.5 & Infinity \\
\hline 2025 & 1 & 22.5 & 17.0 & $(-)$ & 0.5 & Infinity \\
\hline 2025 & 3 & 8.0 & 44.0 & 14 & 0.5 & \\
\hline & & & & & & $2.17 E-05$ \\
\hline
\end{tabular}




\section{ACT REMBCO JOINT VENTURE Ornl Wag 4 Seeps 4 \& 6 Removal Action Project}

In-Situ Hydraulic Conductivity Testing Record

September 13, 1996

\begin{tabular}{|c|c|c|c|c|c|l|}
\hline $\begin{array}{c}\text { Hole } \\
\text { No. }\end{array}$ & $\begin{array}{c}\text { Sleeve } \\
\text { No. }\end{array}$ & $\begin{array}{c}\text { Flow } \\
\text { Rate } \\
\text { (lpm) }\end{array}$ & $\begin{array}{c}\text { Gauge } \\
\text { Pressure } \\
\text { (psi) }\end{array}$ & $\begin{array}{c}\text { Effective } \\
\text { Pressure } \\
\text { (psi) }\end{array}$ & $\begin{array}{c}\text { Length of } \\
\text { Zonested } \\
\text { (m) }\end{array}$ & $\begin{array}{l}\text { Hydraulic Conductivity Value } \\
\text { (m/s) }\end{array}$ \\
\hline 2025 & 2 & 0.5 & 58.0 & 58 & 0.5 & 3.27E-07 \\
\hline 2026 & 1 & 23.0 & 16.0 & $(-)$ & 0.5 & Infinity \\
\hline 2026 & 3 & 23.0 & 20.0 & $(-)$ & 0.5 & Infinity \\
\hline 2026 & 4 & 22.0 & 22.0 & $(-)$ & 0.5 & Infinity \\
\hline 2026 & 5 & 24.0 & 15.0 & $(-)$ & 0.5 & Infinity \\
\hline 2026 & 2 & 4.0 & 50.0 & 40 & 0.5 & \\
\hline 2027 & 1 & 18.0 & 24.0 & $(-)$ & 0.5 & Infinity \\
\hline 2029 & 2 & 15.0 & 28.0 & $(-)$ & 0.5 & Infinity \\
\hline 2029 & 3 & 23.0 & 16.0 & $(-)$ & 0.5 & Infinity \\
\hline 2029 & 5 & 20.0 & 21.0 & $(-)$ & 0.5 & Infinity \\
\hline 2029 & 6 & 12.0 & 32.0 & $(-)$ & 0.5 & Infinity \\
\hline 2029 & 4 & 21.0 & 20.0 & $(-)$ & 0.5 & Infinity \\
\hline 2029 & 1 & 22.0 & 19.0 & $(-)$ & 0.5 & Infinity \\
\hline 2030 & 2 & 18.0 & 28.0 & $(-)$ & 0.5 & Infinity \\
\hline 2030 & 1 & 23.0 & 17.0 & $(-)$ & 0.5 & Infinity \\
\hline 2031 & 2 & 21.0 & 24.0 & $(-)$ & 0.5 & Infinity \\
\hline 2031 & 3 & 23.0 & 20.0 & $(-)$ & 0.5 & Infinity \\
\hline 2031 & 4 & 24.0 & 20.0 & $(-)$ & 0.5 & Infinity \\
\hline 2031 & 5 & 23.0 & 20.0 & $(-)$ & 0.5 & Infinity \\
\hline 2031 & 1 & 23.0 & 18.0 & $(-)$ & 0.5 & Infinity \\
\hline 2031 & 6 & 24.0 & 20.0 & $(-)$ & 0.5 & Infinity \\
\hline 2036 & 9 & 18.0 & 23.0 & $(-)$ & 0.5 & Infinity \\
\hline 2036 & 10 & 19.0 & 23.0 & $(-)$ & 0.5 & Infinity \\
\hline 2036 & 1 & 23.0 & 17.0 & $(-)$ & 0.5 & Infinity \\
\hline 2037 & 3 & 21.0 & 24.0 & $(-)$ & 0.5 & Infinity \\
\hline 2037 & 4 & 22.0 & 24.0 & $(-)$ & 0.5 & Infinity \\
\hline 2037 & 5 & 20.0 & 25.0 & $(-)$ & 0.5 & Infinity \\
\hline 2037 & 2 & 20.0 & 26.0 & $(-)$ & 0.5 & Infinity \\
\hline 2038 & 5 & 14.0 & 31.0 & $(-)$ & 0.5 & Infinity \\
\hline 2038 & 10 & 20.0 & 22.0 & $(-)$ & 0.5 & Infinity \\
\hline 2038 & 8 & 20.0 & 20.0 & $(-)$ & 0.5 & Infinity \\
\hline 2038 & 1 & 23.0 & 18.0 & $(-)$ & 0.5 & Infinity \\
\hline 038 & 7 & 24.0 & 15.0 & $(-)$ & 0.5 & Infinity \\
\hline & & & & & & \\
\hline
\end{tabular}


ACT REMBCO JOINT VENTURE

Ornl Wag 4 Seeps 4 \& 6 Removal Action Project

In-Situ Hydraulic Conductivity Testing Record

September 13, 1996

\begin{tabular}{|c|c|c|c|c|c|l|}
\hline $\begin{array}{c}\text { Hole } \\
\text { No. }\end{array}$ & $\begin{array}{c}\text { Sleeve } \\
\text { No. }\end{array}$ & $\begin{array}{c}\text { Flow } \\
\text { Rate } \\
(\mathrm{pm})\end{array}$ & $\begin{array}{c}\text { Gauge } \\
\text { Pressure } \\
\text { (psi) }\end{array}$ & $\begin{array}{c}\text { Effective } \\
\text { Pressure } \\
\text { (psi) }\end{array}$ & $\begin{array}{c}\text { Length of } \\
\text { Zone Tested } \\
(\mathrm{m})\end{array}$ & $\begin{array}{c}\text { Hydraulic Conductivity Value } \\
\text { (m/s) }\end{array}$ \\
\hline 2039 & 1 & 23.0 & 19.0 & $(-)$ & 0.5 & Infinity \\
\hline 2043 & 1 & 20.0 & 23.0 & $(-)$ & 0.5 & Infinity \\
\hline 2043 & 3 & 21.0 & 26.0 & $(-)$ & 0.5 & Infinity \\
\hline 2043 & 5 & 19.0 & 27.0 & $(-)$ & 0.5 & Infinity \\
\hline 2043 & 4 & 19.0 & 28.0 & $(-)$ & 0.5 & Infinity \\
\hline 2043 & 2 & 21.0 & 26.0 & $(-)$ & 0.5 & Infinity \\
\hline 2045 & 1 & 19.0 & 25.0 & $(-)$ & 0.5 & Infinity \\
\hline 2046 & 5 & 24.0 & 27.0 & $(-)$ & 0.5 & Infinity \\
\hline 2046 & 4 & 20.0 & 21.0 & $(-)$ & 0.5 & Infinity \\
\hline 2046 & 1 & 20.0 & 21.0 & $(-)$ & 0.5 & Infinity \\
\hline 2046 & 3 & 20.0 & 20.0 & $(-)$ & 0.5 & Infinity \\
\hline 2046 & 2 & 21.0 & 20.0 & $(-)$ & 0.5 & Infinity \\
\hline 2047 & 1 & 13.0 & 34.0 & $(-)$ & 0.5 & Infinity \\
\hline 2047 & 2 & 22.0 & 35.0 & $(-)$ & 0.5 & Infinity \\
\hline 2047 & 3 & 24.0 & 31.0 & $(-)$ & 0.5 & Infinity \\
\hline 2047 & 4 & 20.0 & 33.0 & $(-)$ & 0.5 & Infinity \\
\hline 2047 & 5 & 18.0 & 29.0 & $(-)$ & 0.5 & Infinity \\
\hline
\end{tabular}




\section{ACT REMBCO JOINT VENTURE \\ Ornl Wag 4 Seeps 4 \& 6 Removal Action Project \\ In-Situ Hydraulic Conductivity Testing Record}

September 19, 1996

\begin{tabular}{|c|c|c|c|c|c|c|}
\hline $\begin{array}{c}\text { Hole } \\
\text { No. }\end{array}$ & $\begin{array}{c}\text { Sleeve } \\
\text { No. }\end{array}$ & $\begin{array}{c}\text { Flow } \\
\text { Rate } \\
(\mathrm{lpm})\end{array}$ & $\begin{array}{c}\text { Gauge } \\
\text { Pressure } \\
(\mathrm{psi})\end{array}$ & $\begin{array}{c}\text { Effective } \\
\text { Pressure } \\
(\mathrm{psi})\end{array}$ & $\begin{array}{c}\text { Length of } \\
\begin{array}{c}\text { Zone Tested } \\
(\mathrm{m})\end{array}\end{array}$ & $\begin{array}{c}\text { Hydraulic Conductivity Value } \\
(\mathrm{m} / \mathrm{s})\end{array}$ \\
\hline 1012 & 1 & 0.3 & 13.0 & 12.0 & 0.5 & $9.49 \mathrm{E}-07$ \\
\hline 1012 & 2 & 0.9 & 24.8 & 23.8 & 0.5 & $1.44 \mathrm{E}-06$ \\
\hline 1012 & 2 & 3.0 & 35.8 & 33.8 & 0.5 & $3.37 \mathrm{E}-06$ \\
\hline 1012 & 2 & 0.6 & 12.2 & 11.2 & 0.5 & $2.03 \mathrm{E}-06$ \\
\hline 1012 & 3 & 0.7 & 15.0 & 14.0 & 0.5 & $1.90 \mathrm{E}-06$ \\
\hline 1012 & 4 & 1.5 & 15.0 & 14.0 & 0.5 & $4.07 \mathrm{E}-06$ \\
\hline 1012 & 5 & 0.4 & 16.0 & 15.0 & 0.5 & $1.01 \mathrm{E}-06$ \\
\hline 1012 & 6 & 0.4 & 14.6 & 13.6 & 0.5 & $1.12 \mathrm{E}-06$ \\
\hline 1012 & 7 & 2.0 & 15.0 & 14.0 & 0.5 & $5.42 \mathrm{E}-06$ \\
\hline & & & & & & $1.11 \mathrm{E}-06$ \\
\hline 1022 & 1 & 0.3 & 11.3 & 10.3 & 0.5 & $4.22 \mathrm{E}-07$ \\
\hline 1022 & 2 & 0.1 & 10.0 & 9.0 & 0.5 & $0.00 \mathrm{E}+00$ \\
\hline 1022 & 3 & 0.0 & 11.0 & 10.0 & 0.5 & $7.17 \mathrm{E}-06$ \\
\hline 1022 & 4 & 2.4 & 13.7 & 12.7 & 0.5 & $0.00 \mathrm{E}+00$ \\
\hline 1022 & 4 & 0.0 & 25.0 & 25.0 & 0.5 & $4.90 \mathrm{E}-07$ \\
\hline 1022 & 5 & 0.2 & 16.5 & 15.5 & 0.5 & $0.00 \mathrm{E}+00$ \\
\hline 1022 & 6 & 0.0 & 16.0 & 16.0 & 0.5 & $0.00 \mathrm{E}+00$ \\
\hline 1022 & 7 & 0.0 & 14.5 & 14.5 & 0.5 & \\
\hline & & & & & & \\
\hline
\end{tabular}

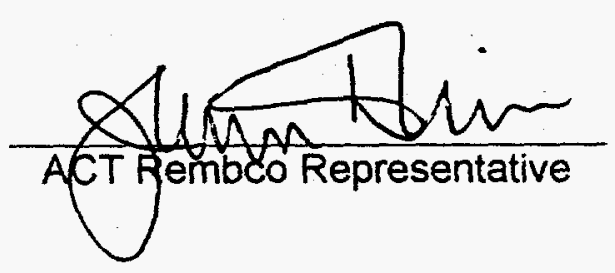




\section{ACT REMBCO JOINT VENTURE \\ Ornl Wag 4 Seeps 4 \& 6 Removal Action Project}

\section{In-Situ Hydraulic Conductivity Testing Record}

September 21, 1996

\begin{tabular}{|c|c|c|c|c|c|c|}
\hline $\begin{array}{c}\text { Hole } \\
\text { No. }\end{array}$ & $\begin{array}{c}\text { Sleeve } \\
\text { No. }\end{array}$ & $\begin{array}{c}\text { Flow } \\
\text { Rate } \\
(\mathrm{pm})\end{array}$ & $\begin{array}{c}\text { Gauge } \\
\text { Pressure } \\
(\mathrm{psi})\end{array}$ & $\begin{array}{c}\text { Effective } \\
\text { Pressure } \\
(\mathrm{psi})\end{array}$ & $\begin{array}{c}\text { Length of } \\
\text { Zone Tested } \\
(\mathrm{m})\end{array}$ & $\begin{array}{c}\text { Hydraulic Conductivity Value } \\
(\mathrm{m} / \mathrm{s})\end{array}$ \\
\hline 1005 & 1,2 & 0.2 & 18.5 & 17.5 & 1 & $2.17 \mathrm{E}-07$ \\
\hline 1005 & 3,4 & 0.1 & 21.0 & 20.0 & 1 & $9.49 \mathrm{E}-08$ \\
\hline 1011 & 1,2 & 0.1 & 24.5 & 23.5 & 1 & $8.07 \mathrm{E}-08$ \\
\hline 1011 & 3,4 & 0.2 & 22.1 & 21.1 & 1 & $1.80 \mathrm{E}-07$ \\
\hline 1013 & 3,4 & 0.1 & 23.5 & 22.5 & 1 & $8.43 \mathrm{E}-08$ \\
\hline 1013 & 1,2 & 0.1 & 24.5 & 23.5 & 1 & $8.07 \mathrm{E}-08$ \\
\hline 1013 & $1-4$ & 0.1 & 26.2 & 25.2 & 2 & $3.76 \mathrm{E}-08$ \\
\hline 1017 & 3,4 & 0.4 & 23.5 & 22.5 & 1 & $3.37 \mathrm{E}-07$ \\
\hline 1017 & 1,2 & 0.2 & 23.0 & 22.0 & 1 & $1.73 \mathrm{E}-07$ \\
\hline 1021 & 1,2 & 0.1 & 25.0 & 24.0 & 1 & $1.91 \mathrm{E}-08$ \\
\hline 1021 & 3,4 & 0.2 & 24.5 & 23.5 & 1 & $1.61 \mathrm{E}-07$ \\
\hline 1038 & 1,2 & 0.2 & 13.9 & 12.9 & 1 & $2.94 \mathrm{E}-07$ \\
\hline 1038 & 3,4 & 0.4 & 11.8 & 10.8 & 1 & $7.03 \mathrm{E}-07$ \\
\hline 1039 & 1,2 & 0.2 & 13.3 & 12.3 & 1 & $1.09 \mathrm{E}-07$ \\
\hline 1039 & 3,4 & 0.1 & 18.5 & 17.5 & 1 & $2.23 \mathrm{E}-07$ \\
\hline 1039 & 5,6 & 0.1 & 9.5 & 8.5 & 1 & $3.67 \mathrm{E}-06$ \\
\hline 1042 & 3,4 & 1.7 & 9.8 & 8.8 & 1 & $4.07 \mathrm{E}-06$ \\
\hline 1042 & 1,2 & 3.3 & 17.4 & 15.4 & 1 & $6.15 \mathrm{E}-07$ \\
\hline 1049 & 3,4 & 0.6 & 19.5 & 18.5 & 1 & $3.22 \mathrm{E}-07$ \\
\hline 1049 & 1,2 & 0.2 & 12.8 & 11.8 & 1 & $6.98 \mathrm{E}-07$ \\
\hline 1049 & 5,6 & 0.6 & 17.3 & 16.3 & 1 & $1.35 \mathrm{E}-05$ \\
\hline 1055 & 3,4 & 5.7 & 10.0 & 8.0 & 1 & $4.59 \mathrm{E}-07$ \\
\hline 1055 & 1,2 & 0.3 & 13.4 & 12.4 & 1 & $4.13 \mathrm{E}-06$ \\
\hline 1061 & 3,4 & 2.5 & 12.5 & 11.5 & 1 & $4.84 \mathrm{E}-07$ \\
\hline 1061 & 1,2 & 0.5 & 20.6 & 19.6 & 1 & \\
\hline & & & & & & 1 \\
\hline
\end{tabular}




\section{ACT REMBCO JOINT VENTURE \\ Ornl Wag 4 Seeps 4 \& 6 Removal Action Project \\ In-Situ Hydraulic Conductivity Testing Record}

September 21, 1996

\begin{tabular}{|c|c|c|c|c|c|c|}
\hline $\begin{array}{c}\text { Hole } \\
\text { No. }\end{array}$ & $\begin{array}{c}\text { Sleeve } \\
\text { No. }\end{array}$ & $\begin{array}{c}\text { Flow } \\
\text { Rate } \\
(\mathrm{pm})\end{array}$ & $\begin{array}{c}\text { Gauge } \\
\text { Pressure } \\
(\mathrm{psi})\end{array}$ & $\begin{array}{c}\text { Effective } \\
\text { Pressure } \\
(\mathrm{psi})\end{array}$ & $\begin{array}{c}\text { Length of } \\
\text { Zone Tested } \\
(\mathrm{m})\end{array}$ & $\begin{array}{c}\text { Hydraulic Conductivity Value } \\
(\mathrm{m} / \mathrm{s})\end{array}$ \\
\hline 1075 & 1,2 & 2.5 & 18.5 & 17.5 & 1 & $2.71 \mathrm{E}-06$ \\
\hline 1075 & 3,4 & 2.0 & 14.0 & 13.0 & 1 & $2.92 \mathrm{E}-06$ \\
\hline 1078 & 3,4 & 0.4 & 18.5 & 17.5 & 1 & $4.34 \mathrm{E}-07$ \\
\hline 1078 & 1,2 & 0.2 & 21.5 & 20.5 & 1 & $1.85 \mathrm{E}-07$ \\
\hline 1080 & 1,2 & 0.2 & 21.5 & 20.5 & 1 & $1.85 \mathrm{E}-07$ \\
\hline
\end{tabular}

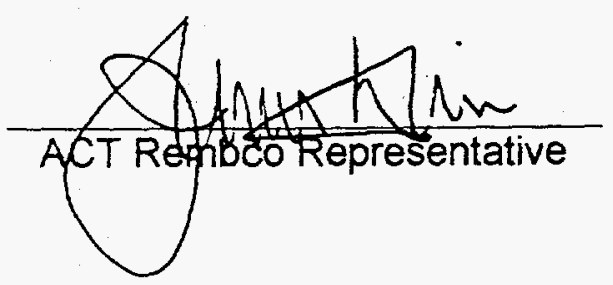




\section{ACT REMBCO JOINT VENTURE Ornl Wag 4 Seeps 4 \& 6 Removal Action Project}

In-Situ Hydraulic Conductivity Testing Record

October 02, 1996

\begin{tabular}{|c|c|c|c|c|c|l|}
\hline $\begin{array}{c}\text { Hole } \\
\text { No. }\end{array}$ & $\begin{array}{c}\text { Sleeve } \\
\text { No. }\end{array}$ & $\begin{array}{c}\text { Flow } \\
\text { Rate } \\
\text { (lpm) }\end{array}$ & $\begin{array}{c}\text { Gauge } \\
\text { Pressure } \\
\text { (psi) }\end{array}$ & $\begin{array}{c}\text { Effective } \\
\text { Pressure } \\
\text { (psi) }\end{array}$ & $\begin{array}{c}\text { Length of } \\
\text { Zone Tested } \\
\text { (m) }\end{array}$ & \multicolumn{1}{|l|}{$\begin{array}{l}\text { Hydraulic Conductivity Value } \\
\text { (m/s) }\end{array}$} \\
\hline 3001 & 1 & 22.0 & 17.0 & $(-)$ & 0.5 & Infinity \\
\hline 3001 & 2 & 19.0 & 13.0 & $(-)$ & 0.5 & Infinity \\
\hline 3001 & 3 & 13.0 & 8.0 & $(-)$ & 0.5 & Infinity \\
\hline 3001 & 4 & 13.0 & 8.0 & $(-)$ & 0.5 & Infinity \\
\hline 3001 & 5 & 30.0 & 25.0 & $(-)$ & 0.5 & Infinity \\
\hline 3001 & 6 & 26.0 & 40.0 & $(-)$ & 0.5 & Infinity \\
\hline 3003 & 4 & 24.0 & 24.0 & $(-)$ & 0.5 & Infinity \\
\hline 3003 & 6 & 17.0 & 13.0 & $(-)$ & 0.5 & Infinity \\
\hline 3003 & 5 & 15.0 & 12.0 & $(-)$ & 0.5 & Infinity \\
\hline 3003 & 3 & 13.0 & 6.0 & $(-)$ & 0.5 & Infinity \\
\hline 3003 & 2 & 16.0 & 24.0 & $(-)$ & 0.5 & Infinity \\
\hline 3003 & 1 & 20.0 & 14.0 & $(-)$ & 0.5 & Infinity \\
\hline 3007 & 1 & 11.0 & 24.0 & $(-)$ & 0.5 & Infinity \\
\hline 3007 & 2 & 21.0 & 35.0 & $(-)$ & 0.5 & Infinity \\
\hline 3007 & 3 & 15.0 & 20.0 & $(-)$ & 0.5 & Infinity \\
\hline 3007 & 4 & 18.0 & 20.0 & $(-)$ & 0.5 & Infinity \\
\hline 3007 & 5 & 23.0 & 32.0 & $(-)$ & 0.5 & Infinity \\
\hline 3009 & 4 & 18.0 & 23.0 & $(-)$ & 0.5 & Infinity \\
\hline 3009 & 2 & 19.0 & 18.0 & $(-)$ & 0.5 & Infinity \\
\hline 3009 & 3 & 25.0 & 30.0 & $(-)$ & 0.5 & Infinity \\
\hline 3009 & 5 & 17.0 & 24.0 & $(-)$ & 0.5 & Infinity \\
\hline 3009 & 1 & 19.0 & 14.0 & $(-)$ & 0.5 & Infinity \\
\hline 3011 & 3 & 29.0 & 32.0 & $(-)$ & 0.5 & Infinity \\
\hline 3011 & 1 & 20.0 & 16.0 & $(-)$ & 0.5 & Infinity \\
\hline 3011 & 2 & 32.0 & 32.0 & $(-)$ & 0.5 & Infinity \\
\hline 3011 & 4 & 27.0 & 21.0 & $(-)$ & 0.5 & Infinity \\
\hline 3014 & 4 & 8.0 & 15.0 & $(-)$ & 0.5 & Infinity \\
\hline 3014 & 3 & 18.0 & 18.0 & $(-)$ & 0.5 & Infinity \\
\hline 3014 & 6 & 1.5 & 66.0 & 65 & 0.5 & \\
\hline 3014 & 1 & 8.0 & 34.0 & 4 & 0.5 & $8.76 E-07$ \\
\hline 3014 & 2 & 17.0 & 17.0 & $(-)$ & 0.5 & Infinity \\
\hline 3014 & 5 & 1.5 & 50.0 & 49 & 0.5 & \\
\hline 3018 & 4 & 14.0 & 22.0 & $(-)$ & 0.5 & Infinity \\
\hline & & & & & & \\
\hline
\end{tabular}




\section{ACT REMBCO JOINT VENTURE Ornl Wag 4 Seeps 4 \& 6 Removal Action Project}

In-Situ Hydraulic Conductivity Testing Record

October 02, 1996

\begin{tabular}{|c|c|c|c|c|c|c|}
\hline $\begin{array}{l}\text { Hole } \\
\text { No. }\end{array}$ & $\begin{array}{l}\text { Sleeve } \\
\text { No. }\end{array}$ & $\begin{array}{l}\text { Flow } \\
\text { Rate } \\
\text { (lpm) }\end{array}$ & $\begin{array}{l}\text { Gauge } \\
\text { Pressure } \\
\text { (psi) }\end{array}$ & $\begin{array}{c}\text { Effective } \\
\text { Pressure } \\
\text { (psi) }\end{array}$ & $\begin{array}{c}\text { Length of } \\
\text { Zone Tested } \\
(\mathrm{m})\end{array}$ & $\begin{array}{l}\text { Hydraulic Conductivity Value } \\
\qquad(\mathrm{m} / \mathrm{s})\end{array}$ \\
\hline 3018 & 3 & 16.0 & 18.0 & $(-)$ & 0.5 & Infinity \\
\hline 3018 & 1 & 11.0 & 33.0 & $(-)$ & 0.5 & Infinity \\
\hline 3018 & 6 & 16.0 & 46.0 & $(-)$ & 0.5 & Infinity \\
\hline 3018 & 2 & 11.0 & 13.0 & $(-)$ & 0.5 & Infinity \\
\hline 3018 & 5 & 13.0 & 17.0 & $(-)$ & 0.5 & Infinity \\
\hline 3020 & 5 & 2.0 & 120.0 & 119 & 0.5 & $6.38 \mathrm{E}-07$ \\
\hline 3020 & 2 & 0.6 & 100.0 & 99 & 0.5 & $2.30 \mathrm{E}-07$ \\
\hline 3020 & 4 & 1.5 & 65.0 & 64 & 0.5 & $8.89 E-07$ \\
\hline 3020 & 3 & 1.9 & 63.0 & 62 & 0.5 & $1.16 \mathrm{E}-06$ \\
\hline 3020 & 1 & 12.0 & 18.0 & $(-)$ & 0.5 & Infinity \\
\hline 3020 & 6 & 2.0 & 75.0 & 74 & 0.5 & $1.03 E-06$ \\
\hline 3021 & 4 & 22.0 & 35.0 & $(-)$ & 0.5 & Infinity \\
\hline 3022 & 1 & 10.0 & 30.0 & $(-)$ & 0.5 & Infinity \\
\hline 3024 & 1. & 25.0 & 20.0 & $\frac{1}{(-)}$ & 0.5 & Infinity \\
\hline 3026 & 1 & 24.0 & 26.0 & $(-)$ & 0.5 & Infinity \\
\hline 3028 & 1 & 11.0 & 11.0 & $(-)$ & 0.5 & Infinity \\
\hline 3030 & 1 & 9.0 & 29.0 & $(-)$ & 0.5 & Infinity \\
\hline 3032 & 1 & 25.0 & 18.0 & $(-)$ & 0.5 & Infinity \\
\hline 3034 & 1 & 14.0 & 40.0 & $(-)$ & 0.5 & Infinity \\
\hline 3036 & 1 & 21.0 & 18.0 & $(-)$ & 0.5 & Infinity \\
\hline 3038 & 1 & 21.0 & 16.0 & $(-)$ & 0.5 & Infinity \\
\hline 3041 & 1 & 12.0 & 36.0 & $(-)$ & 0.5 & Infinity \\
\hline 3044 & 1 & 16.0 & 19.0 & $(-1)$ & 0.5 & Infinity \\
\hline 3048 & 1 & 15.0 & 11.0 & $(-)$ & 0.5 & Infinity \\
\hline 3050 & 1 & 17.0 & 15.0 & $(-)$ & 0.5 & Infinity \\
\hline 4016 & 1 & 13.0 & 43.0 & $(-)$ & 0.5 & Infinity \\
\hline 4016 & 2 & 17.0 & 44.0 & $(-)$ & 0.5 & Infinity \\
\hline 4016 & 3 & 15.0 & 34.0 & $(-)$ & 0.5 & Infinity \\
\hline 4018 & 1 & 9.0 & 48.0 & 18 & 0.5 & 1.90E-05 \\
\hline 4018 & 2 & 1.0 & 50.0 & 49 & 0.5 & $7.75 E-07$ \\
\hline 4021 & 1 & 14.0 & 47.0 & $(-)$ & 0.5 & Infinity \\
\hline 4021 & 2 & 5.0 & 50.0 & 40 & 0.5 & $4.74 E-06$ \\
\hline 021 & 3 & 19.0 & 16.0 & $(-)$ & 0.5 & Infinity \\
\hline
\end{tabular}




\section{ACT REMBCO JOINT VENTURE Ornl Wag 4 Seeps 4 \& 6 Removal Action Project \\ In-Situ Hydraulic Conductivity Testing Record}

October 02, 1996

\begin{tabular}{|c|c|c|c|c|c|l|}
\hline $\begin{array}{c}\text { Hole } \\
\text { No. }\end{array}$ & $\begin{array}{c}\text { Sleeve } \\
\text { No. }\end{array}$ & $\begin{array}{c}\text { Flow } \\
\text { Rate } \\
\text { (lpm) }\end{array}$ & $\begin{array}{c}\text { Gauge } \\
\text { Pressure } \\
\text { (psi) }\end{array}$ & $\begin{array}{c}\text { Effective } \\
\text { Pressure } \\
\text { (psi) }\end{array}$ & $\begin{array}{c}\text { Length of } \\
\text { Zone Tested } \\
(\mathrm{m})\end{array}$ & $\begin{array}{l}\text { Hydraulic Conductivity Value } \\
\text { (m/s) }\end{array}$ \\
\hline 4026 & 4 & 13.0 & 30.0 & $(-)$ & 0.5 & Infinity \\
\hline 4026 & 2 & 16.0 & 21.0 & $(-)$ & 0.5 & Infinity \\
\hline 4026 & 1 & 22.0 & 22.0 & $(-)$ & 0.5 & Infinity \\
\hline 4026 & 3 & 17.0 & 16.0 & $(-)$ & 0.5 & Infinity \\
\hline 4030 & 2 & 18.0 & 23.0 & $(-)$ & 0.5 & Infinity \\
\hline 4030 & 1 & 16.0 & 26.0 & $(-)$ & 0.5 & Infinity \\
\hline 4033 & 2 & 12.0 & 8.0 & $(-)$ & 0.5 & Infinity \\
\hline 4033 & 3 & 23.0 & 30.0 & $(-)$ & 0.5 & Infinity \\
\hline 4033 & 1 & 16.0 & 26.0 & $(-)$ & 0.5 & Infinity \\
\hline 4033 & 4 & 27.0 & 41.0 & $(-)$ & 0.5 & Infinity \\
\hline 4037 & 2 & 8.0 & 26.0 & $(-)$ & 0.5 & Infinity \\
\hline 4037 & 1 & 19.0 & 16.0 & $(-)$ & 0.5 & Infinity \\
\hline 4037 & 4 & 28.0 & 30.0 & $(-)$ & 0.5 & Infinity \\
\hline 4037 & 3 & 9.0 & 29.0 & $(-)$ & 0.5 & Infinity \\
\hline 4042 & 1 & 4.0 & 50.0 & 40 & 0.5 & \\
\hline 4042 & 3 & 22.0 & 34.0 & $(-)$ & 0.5 & Infinity \\
\hline 4042 & 2 & 20.0 & 33.0 & $(-)$ & 0.5 & Infinity \\
\hline 4042 & 4 & 24.0 & 36.0 & $(-)$ & 0.5 & Infinity \\
\hline 4049 & 1 & 17.0 & 17.0 & $(-)$ & 0.5 & Infinity \\
\hline 4049 & 2 & 28.0 & 22.0 & $(-)$ & 0.5 & Infinity \\
\hline 4049 & 3 & 24.0 & 40.0 & $(-)$ & 0.5 & Infinity \\
\hline 4049 & 4 & 34.0 & 40.0 & $(-)$ & 0.5 & Infinity \\
\hline 4052 & 3 & 25.0 & 21.0 & $(-)$ & 0.5 & Infinity \\
\hline 4052 & 2 & 22.0 & 16.0 & $(-)$ & 0.5 & Infinity \\
\hline 4052 & 4 & 22.0 & 13.0 & $(-)$ & 0.5 & Infinity \\
\hline 4052 & 1 & 19.0 & 14.0 & $(-)$ & 0.5 & Infinity \\
\hline 4053 & 1 & 16.0 & 16.0 & $(-)$ & 0.5 & Infinity \\
\hline 4053 & 4 & 25.0 & 20.0 & $(-)$ & 0.5 & Infinity \\
\hline 4054 & 2 & 24.0 & 16.0 & $(-)$ & 0.5 & Infinity \\
\hline 4054 & 1 & 17.0 & 14.0 & $(-)$ & 0.5 & Infinity \\
\hline 4054 & 4 & 25.0 & 23.0 & $(-)$ & 0.5 & Infinity \\
\hline 4054 & 3 & 20.0 & 21.0 & $(-)$ & 0.5 & Infinity \\
\hline 4056 & 1 & 15.0 & 16.0 & $(-)$ & 0.5 & Infinity \\
\hline & & & & & & \\
\hline
\end{tabular}




\section{ACT REMBCO JOINT VENTURE \\ Ornl Wag 4 Seeps 4 \& 6 Removal Action Project \\ In-Situ Hydraulic Conductivity Testing Record}

October 02, 1996

\begin{tabular}{|c|c|c|c|c|c|l|}
\hline $\begin{array}{c}\text { Hole } \\
\text { No. }\end{array}$ & $\begin{array}{c}\text { Sleeve } \\
\text { No. }\end{array}$ & $\begin{array}{c}\text { Flow } \\
\text { Rate } \\
(\mathrm{lpm})\end{array}$ & $\begin{array}{c}\text { Gauge } \\
\text { Pressure } \\
(\mathrm{psi})\end{array}$ & $\begin{array}{c}\text { Effective } \\
\text { Pressure } \\
(\mathrm{psi})\end{array}$ & $\begin{array}{c}\text { Length of } \\
\text { Zone Tested } \\
(\mathrm{m})\end{array}$ & $\begin{array}{c}\text { Hydraulic Conductivity Value } \\
(\mathrm{m} / \mathrm{s})\end{array}$ \\
\hline 4056 & 4 & 17.0 & 20.0 & $(-)$ & 0.5 & Infinity \\
\hline 4056 & 2 & 33.0 & 38.0 & $(-)$ & 0.5 & Infinity \\
\hline 4056 & 3 & 24.0 & 27.0 & $(-)$ & 0.5 & Infinity \\
\hline
\end{tabular}

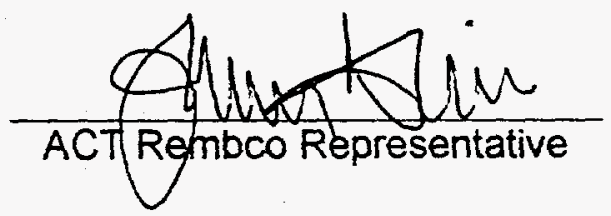




\section{ACT REMBCO JOINT VENTURE Ornl Wag 4 Seeps 4 \& 6 Removal Action Project}

In-Situ Hydraulic Conductivity Testing Record

October 03, 1996

\begin{tabular}{|c|c|c|c|c|c|c|}
\hline $\begin{array}{l}\text { Hole } \\
\text { No. }\end{array}$ & $\begin{array}{l}\text { Sleeve } \\
\text { No. }\end{array}$ & $\begin{array}{l}\text { Flow } \\
\text { Rate } \\
\text { (lpm) }\end{array}$ & $\begin{array}{l}\text { Gauge } \\
\text { Pressure } \\
\text { (psi) }\end{array}$ & \begin{tabular}{|c} 
Effective \\
Pressure \\
(psi)
\end{tabular} & $\begin{array}{l}\text { Length of } \\
\text { Zone Tested } \\
\text { (m) }\end{array}$ & $\begin{array}{l}\text { Hydraulic Conductivity Value } \\
\qquad(\mathrm{m} / \mathrm{s})\end{array}$ \\
\hline 3022 & 6 & 19.0 & 30.0 & $(-1)$ & 0.5 & Infinity \\
\hline 3022 & 5 & 11.0 & 36.0 & $(-)$ & 0.5 & Infinity \\
\hline 3022 & 2 & 13.0 & 33.0 & $(-)$ & 0.5 & Infinity \\
\hline 3022 & 3 & 8.0 & 44.0 & 14 & 0.5 & $2.17 \mathrm{E}-05$ \\
\hline 3022 & 4 & 12.0 & 33.0 & $(-)$ & 0.5 & Infinity \\
\hline 3024 & 6 & 4.0 & 80.0 & 77 & 0.5 & $1.97 \mathrm{E}-06$ \\
\hline 3024 & 2 & 3.0 & 90.0 & 88 & 0.5 & $1.29 \mathrm{E}-06$ \\
\hline 3024 & 5 & 3.0 & 80.0 & 78 & 0.5 & $1.46 \mathrm{E}-06$ \\
\hline 3024 & 3 & 1.0 & 84.0 & 83 & 0.5 & $4.57 \mathrm{E}-07$ \\
\hline 3024 & 4 & 1.0 & 83.0 & 82 & 0.5 & $4.63 \mathrm{E}-07$ \\
\hline 3026 & 5 & 1.0 & 95.0 & 94 & 0.5 & $4.04 \mathrm{E}-07$ \\
\hline 3026 & 2 & 2.0 & 55.0 & 49 & 0.5 & $1.55 \mathrm{E}-06$ \\
\hline 3026 & 6 & 1.0 & 72.0 & 71 & 0.5 & $5.35 \mathrm{E}-07$ \\
\hline 3026 & 3 & 4.0 & 75.0 & 72 & 0.5 & $2.11 \mathrm{E}-06$ \\
\hline 3026 & 4 & 1.0 & 90.0 & 89 & 0.5 & $4.26 \mathrm{E}-07$ \\
\hline 3028 & 5 & 10.0 & 17.0 & $(-)$ & 0.5 & Infinity \\
\hline 3028 & 3 & 19.0 & 19.0 & $(-)$ & 0.5 & Infinity \\
\hline 3028 & 4 & 18.0 & 19.0 & $(-)$ & 0.5 & Infinity \\
\hline 3028 & 2 & 17.0 & 16.0 & $(-)$ & 0.5 & Infinity \\
\hline 3028 & 6 & 16.0 & 20.0 & $(-)$ & 0.5 & Infinity \\
\hline \multicolumn{7}{|c|}{ Packer plugged, tests on various holes must be performed again. } \\
\hline 3020 & 5 & 19.0 & 30.0 & $(-)$ & 0.5 & Infinity \\
\hline 3020 & 2 & 22.0 & 24.0 & $(-)$ & 0.5 & Infinity \\
\hline 3020 & 4 & 23.0 & 23.0 & $(-)$ & 0.5 & Infinity \\
\hline 3020 & 6 & 19.0 & 40.0 & $(-)$ & 0.5 & Infinity \\
\hline 3020 & $\overline{3}$ & 23.0 & 21.0 & $(-)$ & 0.5 & Infinity \\
\hline 3024 & 2 & 12.0 & 6.0 & $(-)$ & 0.5 & Infinity \\
\hline 3024 & $\overline{5}$ & 18.0 & 23.0 & $(-1)$ & 0.5 & Infinity \\
\hline 3024 & 6 & 17.0 & 23.0 & $(-)$ & 0.5 & Infinity \\
\hline 3024 & 4 & 13.0 & 13.0 & $(-)$ & 0.5 & Infinity \\
\hline 3024 & 3 & 13.0 & 7.0 & $(-)$ & 0.5 & Infinity \\
\hline 3026 & 3 & 8.0 & 12.0 & $(-)$ & 0.5 & Infinity \\
\hline 3026 & 2 & 9.0 & 9.0 & $(-)$ & 0.5 & Infinity \\
\hline
\end{tabular}




\section{ACT REMBCO JOINT VENTURE Ornl Wag 4 Seeps 4 \& 6 Removal Action Project}

In-Situ Hydraulic Conductivity Testing Record

October 03, 1996

\begin{tabular}{|c|c|c|c|c|c|c|}
\hline $\begin{array}{l}\text { Hole } \\
\text { No. }\end{array}$ & $\begin{array}{l}\text { Sleeve } \\
\text { No. }\end{array}$ & $\begin{array}{l}\text { Flow } \\
\text { Rate } \\
\text { (lpm) }\end{array}$ & $\begin{array}{c}\text { Gauge } \\
\text { Pressure } \\
\text { (psi) }\end{array}$ & $\begin{array}{c}\text { Effective } \\
\text { Pressure } \\
\text { (psi) }\end{array}$ & $\begin{array}{c}\text { Length of } \\
\text { Zone Tested } \\
\text { (m) }\end{array}$ & $\begin{array}{l}\text { Hydraulic Conductivity Value } \\
\qquad(\mathrm{m} / \mathrm{s})\end{array}$ \\
\hline 3026 & 6 & 14.0 & 32.0 & $(-)$ & 0.5 & Infinity \\
\hline 3026 & 5 & 12.0 & 17.0 & $(-)$ & 0.5 & Infinity \\
\hline 3026 & 4 & 13.0 & 14.0 & $(-1)$ & 0.5 & Infinity \\
\hline 3030 & 4 & 10.0 & 20.0 & $(-1)$ & 0.5 & Infinity \\
\hline 3030 & 3 & 16.0 & 25.0 & $(-)$ & 0.5 & Infinity \\
\hline 3030 & 2 & 11.0 & 40.0 & $(-)$ & 0.5 & Infinity \\
\hline 3030 & 5 & 12.0 & 30.0 & $(-)$ & 0.5 & Infinity \\
\hline 3032 & 3 & 17.0 & 20.0 & $(-1)$ & 0.5 & Infinity \\
\hline 3032 & 2 & 12.0 & 13.0 & $(-1)$ & 0.5 & Infinity \\
\hline 3032 & 6 & 14.0 & 20.0 & $(-)$ & 0.5 & Infinity \\
\hline 3032 & 4 & 19.0 & 26.0 & $(-1)$ & 0.5 & Infinity \\
\hline 3032 & 5 & 13.0 & 19.0 & $(-1)$ & 0.5 & Infinity \\
\hline 3034 & 5 & 12.0 & 6.0 & $(-)$ & 0.5 & Infinity \\
\hline 3034 & 4 & 13.0 & 6.0 & $(-1)$ & 0.5 & Infinity \\
\hline 3034 & 3 & 14.0 & 25.0 & $(-)$ & 0.5 & Infinity \\
\hline 3034 & 6 & 11.0 & 23.0 & $(-)$ & 0.5 & Infinity \\
\hline 3034 & 2 & 13.0 & 20.0 & $(-)$ & 0.5 & Infinity \\
\hline 3036 & 5 & 19.0 & 15.0 & $(-)$ & 0.5 & Infinity \\
\hline 3036 & 6 & 15.0 & 11.0 & $(-1)$ & 0.5 & Infinity \\
\hline 3036 & 4 & 13.0 & 10.0 & $(-)$ & 0.5 & Infinity \\
\hline 3036 & 3 & 14.0 & 11.0 & $(-)$ & 0.5 & Infinity \\
\hline 3036 & 2 & 16.0 & 16.0 & $(-1)$ & 0.5 & Infinity \\
\hline 3038 & 5 & 20.0 & 23.0 & $(-1)$ & 0.5 & Infinity \\
\hline 3038 & 2 & 13.0 & 6.0 & $(-)$ & 0.5 & Infinity \\
\hline 3038 & 4 & 18.0 & 26.0 & $(-)$ & 0.5 & Infinity \\
\hline 3038 & 3 & 15.0 & 15.0 & $(-1)$ & 0.5 & Infinity \\
\hline 3040 & 4 & 18.0 & 13.0 & $(-)$ & 0.5 & Infinity \\
\hline 3040 & 3 & 14.0 & 9.0 & $(-)$ & 0.5 & Infinity \\
\hline 3040 & 2 & 17.0 & 11.0 & $(-1)$ & 0.5 & Infinity \\
\hline 3041 & 5 & 13.0 & 31.0 & $(-)$ & 0.5 & Infinity \\
\hline 3041 & 4 & 16.0 & 42.0 & $(-)$ & 0.5 & Infinity \\
\hline 3041 & 2 & 16.0 & 26.0 & $(-1)$ & 0.5 & Infinity \\
\hline 041 & 3 & 15.0 & 35.0 & $(-)$ & 0.5 & Infinity \\
\hline
\end{tabular}




\section{ACT REMBCO JOINT VENTURE \\ Ornl Wag 4 Seeps 4 \& 6 Removal Action Project}

In-Situ Hydraulic Conductivity Testing Record

October 03, 1996

\begin{tabular}{|c|c|c|c|c|c|c|}
\hline $\begin{array}{l}\text { Hole } \\
\text { No. }\end{array}$ & $\begin{array}{l}\text { Sleeve } \\
\text { No. }\end{array}$ & $\begin{array}{l}\text { Flow } \\
\text { Rate } \\
(\mathrm{lpm})\end{array}$ & $\begin{array}{c}\text { Gauge } \\
\text { Pressure } \\
\text { (psi) }\end{array}$ & $\begin{array}{l}\text { Effective } \\
\text { Pressure } \\
\text { (psi) }\end{array}$ & $\begin{array}{l}\text { Length of } \\
\text { Zone Tested } \\
\text { (m) }\end{array}$ & $\begin{array}{l}\text { Hydraulic Conductivity Value } \\
(\mathrm{m} / \mathrm{s})\end{array}$ \\
\hline 3044 & 4 & 12.0 & 18.0 & $(-)$ & 0.5 & Infinity \\
\hline 3044 & 2 & 8.0 & 7.0 & $(-1)$ & 0.5 & Infinity \\
\hline 3044 & 5 & 16.0 & 20.0 & $(-)$ & 0.5 & Infinity \\
\hline 3044 & 6 & 17.0 & 20.0 & $(-)$ & 0.5 & Infinity \\
\hline 3044 & 3 & 17.0 & 18.0 & $(-1)$ & 0.5 & Infinity \\
\hline 3045 & 2 & 17.0 & 14.0 & $(-)$ & 0.5 & Infinity \\
\hline 3045 & 3 & 19.0 & 15.0 & $(-)$ & 0.5 & Infinity \\
\hline 3048 & 2 & 19.0 & 17.0 & $(-)$ & 0.5 & Infinity \\
\hline 3048 & 3 & 17.0 & 19.0 & $(-)$ & 0.5 & Infinity \\
\hline 3050 & 2 & 17.0 & 15.0 & $\frac{1}{(-)}$ & 0.5 & Infinity \\
\hline 3050 & 3 & 16.0 & 11.0 & $(-)$ & 0.5 & Infinity \\
\hline 4013 & $\frac{5}{2}$ & 15.0 & 13.0 & $\frac{1-1}{(-)}$ & 0.5 & Infinity \\
\hline 4034 & 1 & 19.0 & 19.0 & $(-)$ & 0.5 & Infinity \\
\hline 4034 & $\frac{1}{2}$ & 16.0 & 15.0 & $\frac{(-1}{(-)}$ & 0.5 & Infinity \\
\hline 4047 & 2 & 16.0 & 24.0 & $(-)$ & 0.5 & Infinity \\
\hline 4047 & 3 & 16.0 & 27.0 & $(-)$ & 0.5 & Infinity \\
\hline 4047 & 4 & 15.0 & 22.0 & $(-)$ & 0.5 & Infinity \\
\hline 4047 & 1 & 15.0 & 30.0 & $(-)$ & 0.5 & Infinity \\
\hline
\end{tabular}




\section{ACT REMBCO JOINT VENTURE \\ Ornl Wag 4 Seeps 4 \& 6 Removal Action Project \\ In-Situ Hydraulic Conductivity Testing Record}

October 09, 1996

\begin{tabular}{|c|c|c|c|c|c|c|}
\hline $\begin{array}{l}\text { Hole } \\
\text { No. }\end{array}$ & $\begin{array}{l}\text { Sleeve } \\
\text { No. }\end{array}$ & $\begin{array}{l}\text { Flow } \\
\text { Rate } \\
\text { (lpm) }\end{array}$ & $\begin{array}{l}\text { Gauge } \\
\text { Pressure } \\
\text { (psi) }\end{array}$ & $\begin{array}{c}\text { Effective } \\
\text { Pressure } \\
\text { (psi) }\end{array}$ & $\begin{array}{c}\text { Length of } \\
\text { Zone Tested } \\
\text { (m) }\end{array}$ & $\begin{array}{l}\text { Hydraulic Conductivity Value } \\
\qquad(\mathrm{m} / \mathrm{s})\end{array}$ \\
\hline 2046 & $1-5$ & 0.0 & 10.0 & 10.0 & 6 & $<1.0 E-08$ \\
\hline 2036 & $1-8$ & \multicolumn{5}{|c|}{ No volume recorded on flowmeter after 5 minutes. } \\
\hline 2024 & $1-6$ & 00 & 116 & 116 & 55 & $<10$ E-08 \\
\hline 2031 & $1-6$ & \multicolumn{5}{|c|}{ No volume recorded on flowmeter after 5 minutes. } \\
\hline & & & & & & \\
\hline 2013 & $1-5$ & 0.0 & 9.7 & 9.7 & 4.5 & $<1.0 \mathrm{E}-08$ \\
\hline 2002 & $1-5$ & \multicolumn{5}{|c|}{ No volume recorded on flowmeter after 5 minutes. } \\
\hline & & & & & & \\
\hline 1002 & $1-4$ & 0.0 & 9.5 & 9.5 & 4 & $<1.0 \mathrm{E}-08$ \\
\hline 1012 & $1-5$ & \multicolumn{5}{|c|}{ No volume recorded on flowmeter after 5 minutes. } \\
\hline & & & & & & \\
\hline 1022 & $1-5$ & 0.0 & 9.9 & 9.9 & 4.5 & $<1.0$ E-08 \\
\hline 1032 & $1-5$ & \multicolumn{5}{|c|}{ No volume recorded on flowmeter after 5 minutes. } \\
\hline
\end{tabular}

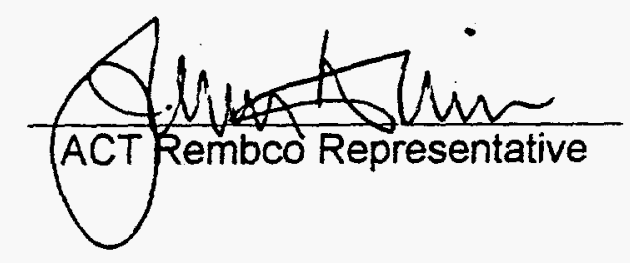




\section{ACT REMBCO JOINT VENTURE \\ Ornl Wag 4 Seeps 4 \& 6 Removal Action Project \\ In-Situ Hydraulic Conductivity Testing Record}

October 10, 1996

\begin{tabular}{|c|c|c|c|c|c|c|}
\hline $\begin{array}{l}\text { Hole } \\
\text { No. }\end{array}$ & $\begin{array}{l}\text { Sleeve } \\
\text { No. }\end{array}$ & $\begin{array}{l}\text { Flow } \\
\text { Rate } \\
\text { (Ipm) }\end{array}$ & \begin{tabular}{|c} 
Gauge \\
Pressure \\
(psi)
\end{tabular} & $\begin{array}{l}\text { Effective } \\
\text { Pressure } \\
\text { (psi) }\end{array}$ & $\begin{array}{l}\text { Length of } \\
\text { Zone Tested } \\
\text { (m) }\end{array}$ & $\begin{array}{l}\text { Hydraulic Conductivity Value } \\
\qquad(\mathrm{m} / \mathrm{s})\end{array}$ \\
\hline 1201 & $1-5$ & 0.0 & 9.8 & 9.8 & 4.5 & $<1.0$ E-08 \\
\hline 1202 & $1-5$ & \multicolumn{5}{|c|}{ No volume recorded on flowmeter after 5 minutes. } \\
\hline 1042 & $1-3$ & 0.0 & 99 & 99 & 3.5 & $<10 F-08$ \\
\hline 1055 & $1-5$ & \multicolumn{5}{|c|}{ No volume recorded on flowmeter after 5 minutes. } \\
\hline 1075 & $1-5$ & 0.0 & 9.8 & 9.8 & 2.25 & $<1.0$ E-08 \\
\hline & & \multicolumn{5}{|c|}{ No volume recorded on flowmeter after 5 minutes. } \\
\hline
\end{tabular}

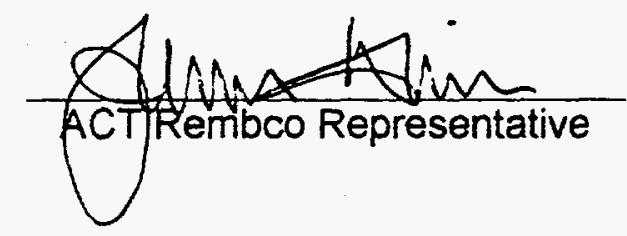




\section{ACT REMBCO JOINT VENTURE \\ Ornl Wag 4 Seeps 4 \& 6 Removal Action Project}

In-Situ Hydraulic Conductivity Testing Record

October 23, 1996

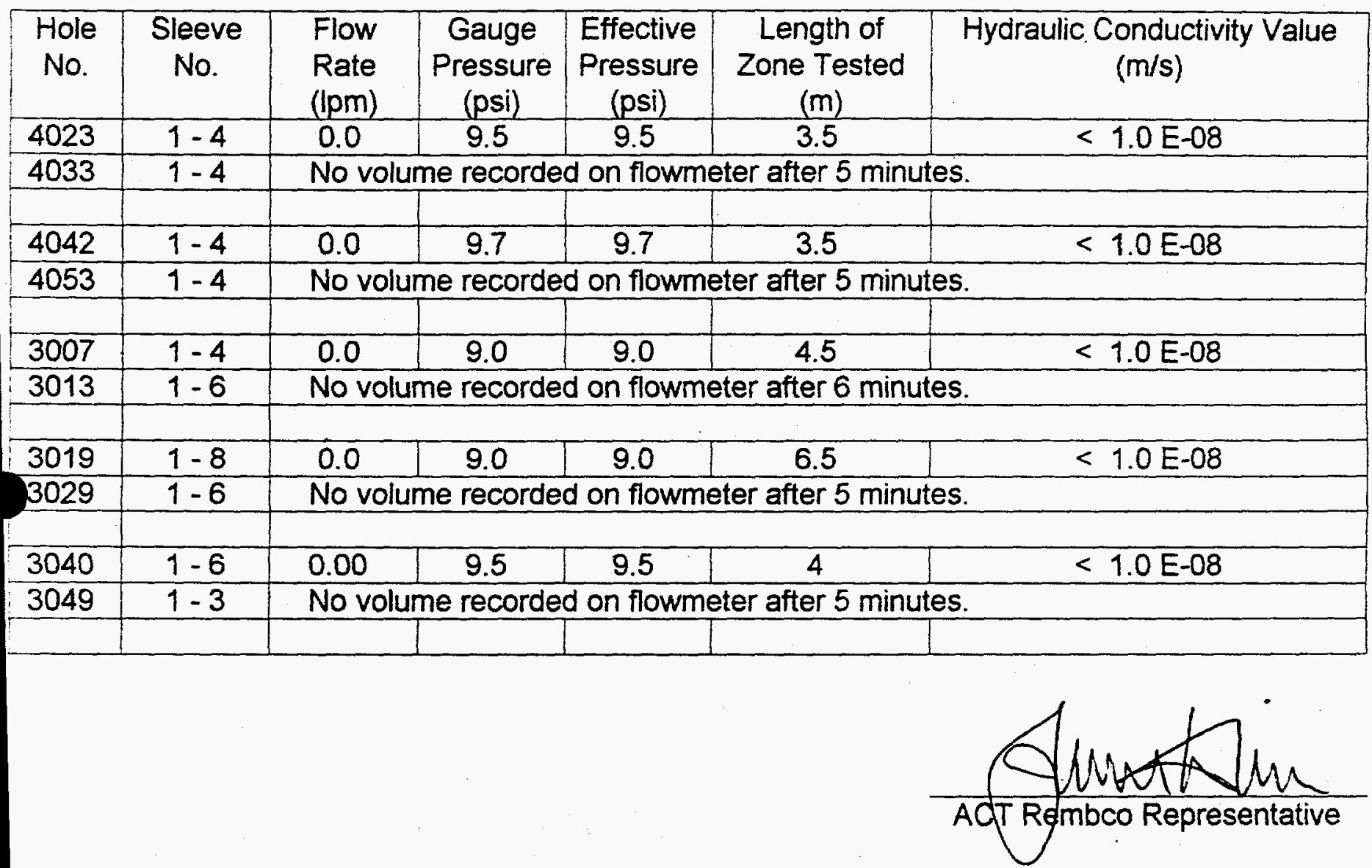




\section{APPENDIX D}

GROUT QUALITY CONTROL TESTING RECORDS 


\section{CEMENTITIOUS GROUT \\ QUALITY CONTROL TESTING REPORTS}




\section{ACT REMBCO JOINT VENTURE \\ Ornl Wag 4 Seeps 4 \& 6 Removal Action Project}

Quality Contol Report

August 13, 1996

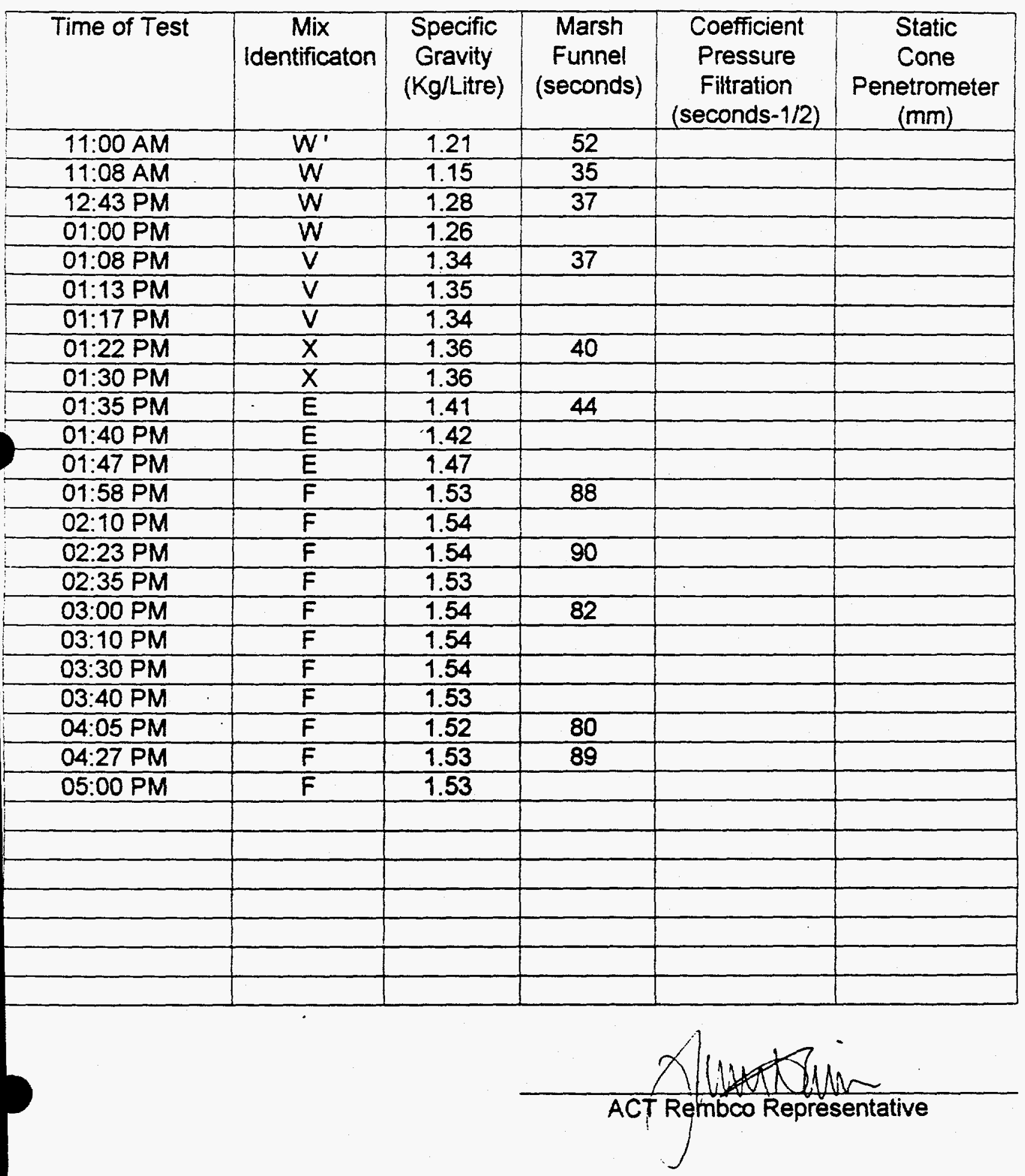


ACT REMBCO JOINT VENTURE

Ornl Wag 4 Seeps 4 \& 6 Removal Action Project

Quality Contol Report

August 14, 1996

\begin{tabular}{|c|c|c|c|c|c|}
\hline Time of Test & $\begin{array}{c}\text { Mix } \\
\text { Identificaton }\end{array}$ & $\begin{array}{c}\text { Specific } \\
\text { Gravity } \\
\text { (Kg/Litre) }\end{array}$ & $\begin{array}{c}\text { Marsh } \\
\text { Funnel } \\
\text { (seconds) }\end{array}$ & $\begin{array}{l}\text { Coefficient } \\
\text { Pressure } \\
\text { Filtration } \\
\text { (seconds-1/2) }\end{array}$ & $\begin{array}{c}\text { Static } \\
\text { Cone } \\
\text { Penetrometer } \\
(\mathrm{mm})\end{array}$ \\
\hline $07: 53 \mathrm{AM}$ & W & 1.28 & 38 & & \\
\hline $08: 00 \mathrm{AM}$ & $\bar{W}$ & 1.28 & 38 & & \\
\hline $08: 05 \mathrm{AM}$ & $\bar{W}$ & 1.28 & & & \\
\hline $08: 10 \mathrm{AM}$ & $\bar{W}$ & 1.31 & & & \\
\hline $08: 21 \mathrm{AM}$ & $\bar{W}$ & 1.29 & & & \\
\hline $08: 26 \mathrm{AM}$ & $\bar{x}$ & 1.38 & 43 & & \\
\hline $08: 31 \mathrm{AM}$ & $\bar{x}$ & 1.38 & 43 & & \\
\hline $08: 37 \mathrm{AM}$ & $\bar{x}$ & 1.39 & 42 & & \\
\hline $08: 46 \mathrm{AM}$ & $x$ & 1.38 & 42 & & \\
\hline $08: 58$ AM & $E$ & 1.45 & 56 & & \\
\hline $09: 02$ AM & $E$ & 1.45 & & & \\
\hline $09: 16 \mathrm{AM}$ & $E$ & 1.47 & 65 & & \\
\hline $09: 27 \mathrm{AM}$ & $E$ & 1.44 & 57 & & \\
\hline $09: 36 \mathrm{AM}$ & $\bar{E}$ & 1.45 & 57 & & \\
\hline $09: 55 \mathrm{AM}$ & $F^{\prime}$ & 1.57 & 72 & & \\
\hline $10: 46 \mathrm{AM}$ & $F^{\prime}$ & 1.57 & 80 & & \\
\hline $11: 05 \mathrm{AM}$ & $F^{\prime}$ & 1.54 & 82 & & \\
\hline $11: 22 \mathrm{AM}$ & $F^{\prime}$ & 1.54 & 100 & & \\
\hline $11: 50 \mathrm{AM}$ & $F^{\prime}$ & 1.55 & 95 & & \\
\hline $01: 16 \mathrm{PM}$ & $F^{\prime}$ & 1.54 & 85 & & \\
\hline & - & & - & & \\
\hline & & & & & \\
\hline & & & & & \\
\hline & & & & & \\
\hline & & & & & \\
\hline & & & & & \\
\hline & & & & & \\
\hline & & & & & \\
\hline & & & & & \\
\hline
\end{tabular}




\section{ACT REMBCO JOINT VENTURE \\ Ornl Wag 4 Seeps 4 \& 6 Removal Action Project}

Quality Contol Report

August 15, 1996

\begin{tabular}{|c|c|c|c|c|c|}
\hline Time of Test & \begin{tabular}{c|} 
Mix \\
Identificaton
\end{tabular} & $\begin{array}{l}\text { Specific } \\
\text { Gravity } \\
\text { (Kg/Litre) }\end{array}$ & $\begin{array}{c}\text { Marsh } \\
\text { Funnel } \\
\text { (seconds) }\end{array}$ & $\begin{array}{l}\text { Coefficient } \\
\text { Pressure } \\
\text { Filtration } \\
\text { (seconds-1/2) }\end{array}$ & $\begin{array}{c}\text { Static } \\
\text { Cone } \\
\text { Penetrometer } \\
(\mathrm{mm}) \\
\end{array}$ \\
\hline $01: 22 \mathrm{PM}$ & $\bar{W}$ & 1.27 & 41 & & \\
\hline $01: 27 \mathrm{PM}$ & W & 1.26 & & & \\
\hline $01: 34$ PM & $\bar{W}$ & 1.25 & & & \\
\hline $01: 50 \mathrm{PM}$ & $\bar{W}$ & 1.26 & & & \\
\hline $02: 32 \mathrm{PM}$ & W & 1.26 & 43 & & \\
\hline $02: 38 \mathrm{PM}$ & W & 1.26 & & & \\
\hline $02: 45 \mathrm{PM}$ & $\bar{W}$ & 1.26 & & & \\
\hline $02: 56 \mathrm{PM}$ & $\bar{W}$ & 1.26 & & & \\
\hline $03: 10 \mathrm{PM}$ & $\bar{x}$ & 1.40 & 44 & & \\
\hline $03: 16 \mathrm{PM}$ & $\bar{x}$ & 1.40 & & & \\
\hline $03: 24 \mathrm{PM}$ & $\bar{x}$ & 1.40 & 46 & & \\
\hline & & & & & \\
\hline & & & & & \\
\hline & & & & & \\
\hline & & & & & \\
\hline & & & & & \\
\hline & & & & & \\
\hline & & & & & \\
\hline & & & & & \\
\hline & & & & & \\
\hline & & & & & \\
\hline & & & & & \\
\hline & & & & & \\
\hline & & & & & \\
\hline & & & & & \\
\hline & & & & 4 & \\
\hline & & & & erepr & e \\
\hline
\end{tabular}




\section{ACT REMBCO JOINT VENTURE \\ Ornl Wag 4 Seeps 4 \& 6 Removal Action Project}

Quality Contol Report

August 19, 1996

\begin{tabular}{|c|c|c|c|c|c|}
\hline Time of Test & $\begin{array}{c}\text { Mix } \\
\text { Identificaton }\end{array}$ & $\begin{array}{c}\text { Specific } \\
\text { Gravity } \\
\text { (Kg/Litre) }\end{array}$ & $\begin{array}{c}\text { Marsh } \\
\text { Funnel } \\
\text { (seconds) }\end{array}$ & $\begin{array}{l}\text { Coefficient } \\
\text { Pressure } \\
\text { Filtration } \\
\text { (seconds-1/2) }\end{array}$ & $\begin{array}{c}\text { Static } \\
\text { Cone } \\
\text { Penetrometer } \\
(\mathrm{mm})\end{array}$ \\
\hline $09: 44 \mathrm{AM}$ & $\bar{W}$ & 1.30 & 40 & & \\
\hline $09: 50 \mathrm{AM}$ & $\bar{W}$ & 1.29 & 39 & & \\
\hline $10: 05 \mathrm{AM}$ & $\bar{W}$ & 1.29 & & & \\
\hline $10: 09 \mathrm{AM}$ & $\bar{W}$ & 1.29 & & & \\
\hline $10: 15 \mathrm{AM}$ & $\bar{x}$ & 1.38 & 48 & & \\
\hline $10: 21 \mathrm{AM}$ & $\bar{x}$ & 1.38 & 48 & & \\
\hline $10: 27 \mathrm{AM}$ & $\bar{x}$ & 1.38 & & & \\
\hline $10: 32 \mathrm{AM}$ & $\bar{x}$ & 1.38 & & & \\
\hline $10: 45 \mathrm{AM}$ & $E$ & 1.44 & & & \\
\hline $10: 49 \mathrm{AM}$ & $E$ & 1.44 & & & \\
\hline $10: 53 \mathrm{AM}$ & $E$ & 1.44 & 55 & & \\
\hline $11: 02 \mathrm{AM}$ & $E$ & 1.45 & & & \\
\hline $11: 10 \mathrm{AM}$ & $E$ & 1.44 & & & \\
\hline $11: 15 \mathrm{AM}$ & $E$ & 1.44 & & & \\
\hline $11: 34 \mathrm{AM}$ & $E$ & 1.45 & 58 & & \\
\hline $11: 45 \mathrm{AM}$ & $E$ & 1.45 & & & \\
\hline $11: 56 \mathrm{AM}$ & $E$ & 1.44 & & & \\
\hline $12: 48 \mathrm{PM}$ & $E$ & 1.44 & & & \\
\hline 01:05 PM & $E$ & 1.44 & & & \\
\hline $01: 20 P M$ & $E$ & 1.44 & 57 & & \\
\hline 01:38 PM & $\bar{E}$ & 1.44 & & & \\
\hline $01: 50 \mathrm{PM}$ & $E$ & 1.45 & & & \\
\hline $02: 00 \mathrm{PM}$ & $E$ & 1.44 & & & \\
\hline 02:09 PM & $\bar{E}$ & 1.44 & & & \\
\hline $02: 23 \mathrm{PM}$ & $\bar{E}$ & 1.46 & 65 & & $\cdot$ \\
\hline $02: 30 \mathrm{PM}$ & $x$ & 1.38 & & & \\
\hline $02: 50 \mathrm{PM}$ & $x$ & 1.38 & 44 & & \\
\hline $03: 14 \mathrm{PM}$ & $E$ & 1.43 & & & \\
\hline $03: 23 \mathrm{PM}$ & $E$ & 1.44 & 58 & & \\
\hline $03: 48 \mathrm{PM}$ & $E$ & 1.44 & 56 & & \\
\hline
\end{tabular}




\section{ACT REMBCO JOINT VENTURE \\ Ornl Wag 4 Seeps 4 \& 6 Removal Action Project \\ Quality Contol Report \\ August 20,1996}

\begin{tabular}{|c|c|c|c|c|c|}
\hline Time of Test & $\begin{array}{c}\text { Mix } \\
\text { Identificaton }\end{array}$ & $\begin{array}{c}\text { Specific } \\
\text { Gravity } \\
\text { (Kg/Litre) }\end{array}$ & $\begin{array}{c}\text { Marsh } \\
\text { Funnel } \\
\text { (seconds) }\end{array}$ & $\begin{array}{l}\text { Coefficient } \\
\text { Pressure } \\
\text { Filtration } \\
\text { (seconds-1/2) }\end{array}$ & $\begin{array}{c}\text { Static } \\
\text { Cone } \\
\text { Penetrometer } \\
(\mathrm{mm})\end{array}$ \\
\hline 12:40 PM & $x$ & 1.39 & 44 & & \\
\hline $12: 47 \mathrm{PM}$ & $x$ & 1.39 & & & \\
\hline 12:50 PM & $x$ & 1.39 & & & \\
\hline $12: 52 \mathrm{PM}$ & $x$ & 1.38 & & & \\
\hline $01: 20 \mathrm{PM}$ & $x$ & 1.40 & 50 & & \\
\hline $01: 26 \mathrm{PM}$ & $\bar{x}$ & 1.37 & 44 & & \\
\hline $01: 34$ PM & $x$ & 1.38 & & & \\
\hline 01:40 PM & $E$ & 1.46 & & & \\
\hline $01: 45 \mathrm{PM}$ & $E$ & 1.48 & 54 & & \\
\hline 01:52 PM & $E$ & 1.44 & & & \\
\hline $01: 55 \mathrm{PM}$ & $E$ & 1.45 & & & \\
\hline $02: 00 \mathrm{PM}$ & $E$ & 1.46 & 59 & & \\
\hline $02: 05 \mathrm{PM}$ & $E$ & 1.48 & & & \\
\hline $02: 07 \mathrm{PM}$ & $E$ & 1.45 & & & \\
\hline $02: 14$ PM & $F$ & 1.54 & 90 & & \\
\hline $02: 18 \mathrm{PM}$ & $F$ & 1.53 & & & \\
\hline $02: 23 \mathrm{PM}$ & $F$ & 1.57 & 106 & & \\
\hline $02: 35 \mathrm{PM}$ & $\bar{x}$ & 1.39 & & & \\
\hline $02: 39 \mathrm{PM}$ & $\bar{x}$ & 1.38 & & & \\
\hline $02: 57 \mathrm{PM}$ & $x$ & 1.38 & & & \\
\hline 03:07 PM & $x$ & 1.39 & 50 & & \\
\hline $03: 13 \mathrm{PM}$ & $\bar{x}$ & 1.36 & & & \\
\hline $03: 16 \mathrm{PM}$ & $x$ & 1.38 & & & \\
\hline $03: 23 \mathrm{PM}$ & $\bar{x}$ & 1.35 & & & \\
\hline $03: 28 \mathrm{PM}$ & $\bar{x}$ & 1.37 & 43 & & \\
\hline $03: 40 \mathrm{PM}$ & $E$ & 1.46 & & & \\
\hline 03:47 PM & $E$ & 1.44 & 56 & & \\
\hline 03:51 PM & $E$ & 1.45 & & & \\
\hline $03: 59 \mathrm{PM}$ & $E$ & 1.42 & & & \\
\hline $04: 18 \mathrm{PM}$ & $E$ & 1.45 & 51 & & \\
\hline 04:27 PM & $E$ & 1.44 & & 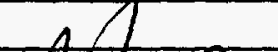 & \\
\hline
\end{tabular}


ACT REMBCO JOINT VENTURE

Ornl Wag 4 Seeps 4 \& 6 Removal Action Project

Quality Contol Report

August 21, 1996

\begin{tabular}{|c|c|c|c|c|c|}
\hline Time of Test & $\begin{array}{c}\text { Mix } \\
\text { Identificaton }\end{array}$ & $\begin{array}{c}\text { Specific } \\
\text { Gravity } \\
\text { (Kg/Litre) }\end{array}$ & $\begin{array}{c}\text { Marsh } \\
\text { Funnel } \\
\text { (seconds) }\end{array}$ & $\begin{array}{c}\text { Coefficient } \\
\text { Pressure } \\
\text { Filtration } \\
\text { (seconds-1/2) } \\
\end{array}$ & $\begin{array}{c}\text { Static } \\
\text { Cone } \\
\text { Penetrometer } \\
(\mathrm{mm})\end{array}$ \\
\hline 09:03 AM & $\bar{x}$ & 1.35 & & & \\
\hline $09: 10 \mathrm{AM}$ & $x$ & 1.37 & & & \\
\hline $09: 15 \mathrm{AM}$ & $\bar{x}$ & 1.38 & 46 & & \\
\hline $09: 25 \mathrm{AM}$ & $E$ & 1.47 & & & \\
\hline $09: 35 \mathrm{AM}$ & $E$ & 1.44 & 58 & & \\
\hline $09: 48 \mathrm{AM}$ & $E$ & 1.46 & & & \\
\hline $09: 53 \mathrm{AM}$ & $E$ & 1.43 & & & \\
\hline $10: 01 \mathrm{AM}$ & $E$ & 1.45 & 52 & & \\
\hline $10: 15 \mathrm{AM}$ & $F$ & 1.55 & & & \\
\hline 10:19 AM & $F$ & 1.51 & 80 & & \\
\hline $10: 25 \mathrm{AM}$ & $\bar{F}$ & 1.54 & & & \\
\hline $10: 34 \mathrm{AM}$ & $F$ & 1.53 & & & \\
\hline $10: 39 \mathrm{AM}$ & $F$ & 1.55 & & & \\
\hline $10: 43 \mathrm{AM}$ & $F$ & 1.54 & 97 & & \\
\hline $11: 00 \mathrm{AM}$ & $E$ & 1.44 & & & \\
\hline $11: 07 \mathrm{AM}$ & $E$ & 1.48 & & & \\
\hline $11: 13 \mathrm{AM}$ & $E$ & 1.45 & & & \\
\hline $11: 22 \mathrm{AM}$ & $\bar{E}$ & 1.46 & & & \\
\hline $11: 39 \mathrm{AM}$ & $E$ & 1.43 & & & \\
\hline $11: 47 \mathrm{AM}$ & $E$ & 1.45 & 53 & & \\
\hline $12: 55 \mathrm{PM}$ & $x$ & 1.33 & 41 & & \\
\hline $01: 05 \mathrm{PM}$ & $\bar{x}$ & 1.38 & & & \\
\hline $01: 11 \mathrm{PM}$ & $x$ & 1.37 & & & \\
\hline $01: 18 P M$ & $\bar{x}$ & 1.36 & 46 & & \\
\hline $01: 30 \mathrm{PM}$ & $\bar{x}$ & 1.38 & & & \\
\hline $01: 50 \mathrm{PM}$ & $x$ & 1.39 & 47 & & \\
\hline 02:10 PM & $E$ & 1.44 & & & \\
\hline $02: 18 \mathrm{PM}$ & $F$ & 1.55 & 108 & & \\
\hline $02: 32 \mathrm{PM}$ & $\bar{F}$ & 1.54 & & & \\
\hline $02: 44 \mathrm{PM}$ & $F$ & 1.52 & & & \\
\hline 03:00 PM & $F$ & 1.56 & 118 & & \\
\hline $03: 16 \mathrm{PM}$ & $F$ & 1.55 & & $\Omega$ & \\
\hline 03:27 PM & $F$ & 1.53 & 77 & & \\
\hline
\end{tabular}




\section{ACT REMBCO JOINT VENTURE \\ Ornl Wag 4 Seeps 4 \& 6 Removal Action Project}

Quality Contol Report

August 22, 1996

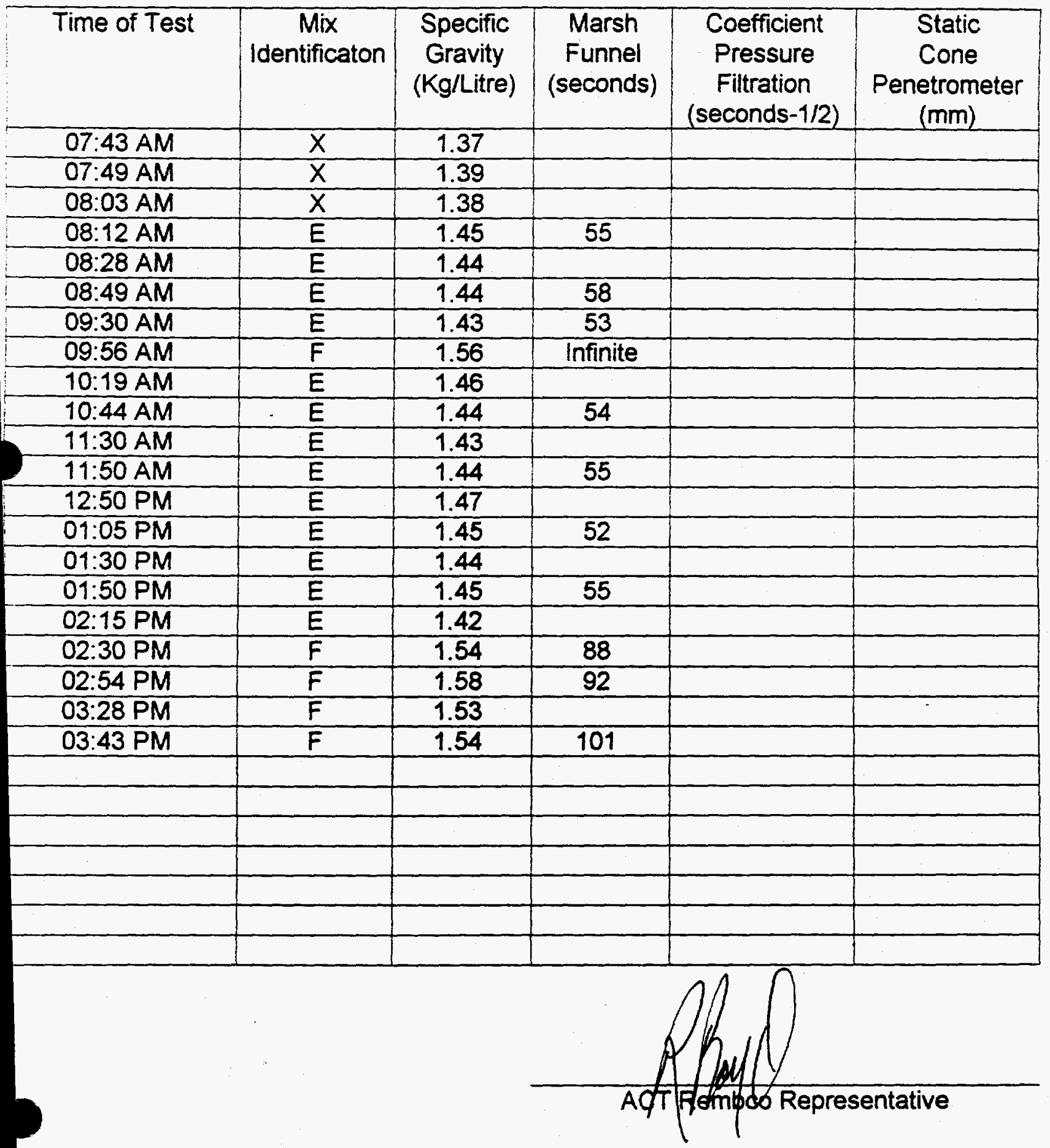




\section{ACT REMBCO JOINT VENTURE Ornl Wag 4 Seeps 4 \& 6 Removal Action Project}

Quality Contol Report

August 23, 1996

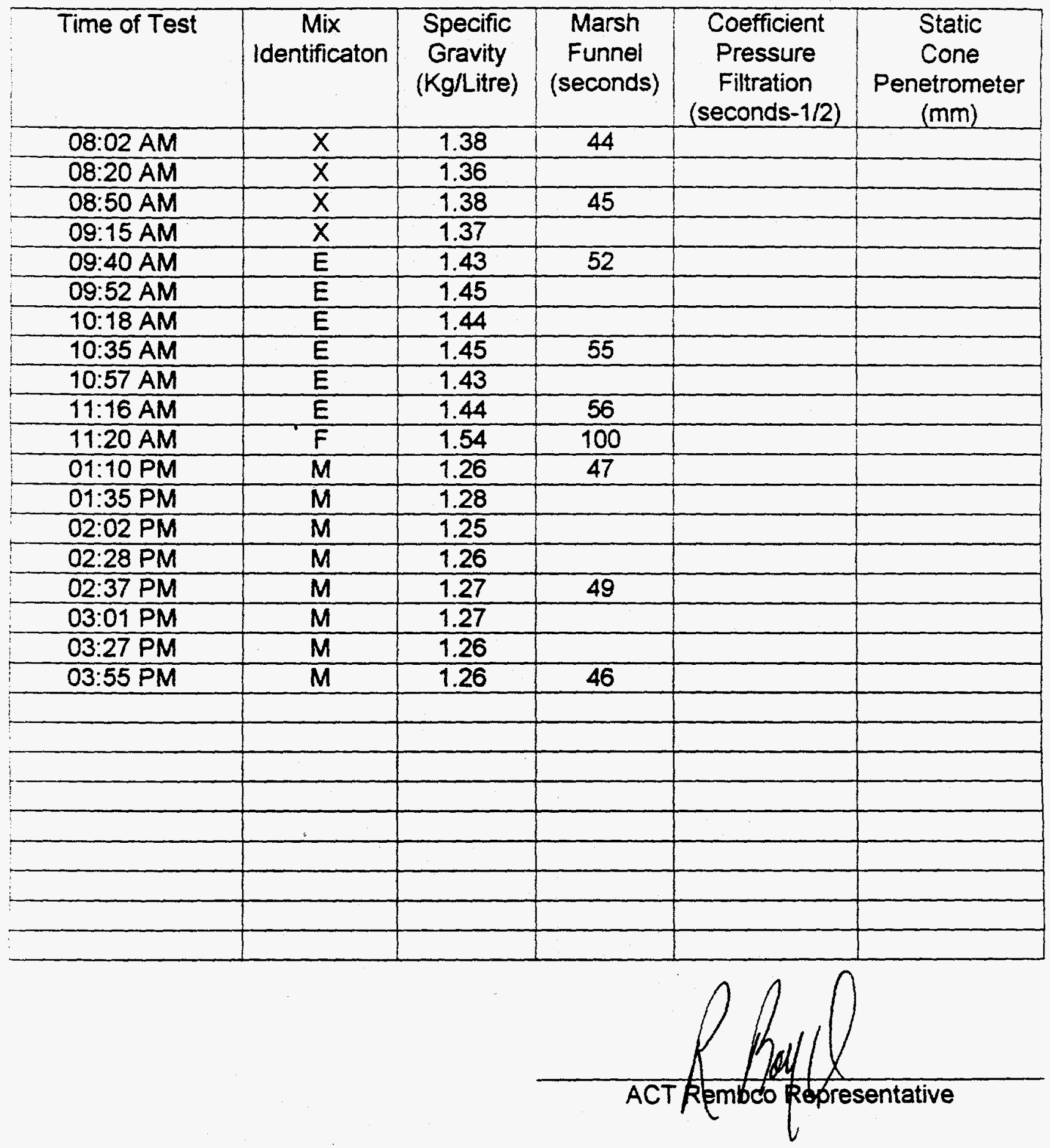




\section{ACT REMBCO JOINT VENTURE Ornl Wag 4 Seeps 4 \& 6 Removal Action Project \\ Quality Contol Report \\ August 26, 1996}

\begin{tabular}{|c|c|c|c|c|c|}
\hline Time of Test & $\begin{array}{c}\text { Mix } \\
\text { Identificaton }\end{array}$ & $\begin{array}{l}\text { Specific } \\
\text { Gravity } \\
\text { (Kg/Litre) }\end{array}$ & $\begin{array}{c}\text { Marsh } \\
\text { Funnel } \\
\text { (seconds) }\end{array}$ & $\begin{array}{l}\text { Coefficient } \\
\text { Pressure } \\
\text { Filtration } \\
\text { (seconds-1/2) }\end{array}$ & $\begin{array}{c}\text { Static } \\
\text { Cone } \\
\text { Penetrometer } \\
(\mathrm{mm})\end{array}$ \\
\hline $08: 20 \mathrm{AM}$ & $\bar{x}$ & 1.37 & 44 & & \\
\hline $08: 47 \mathrm{AM}$ & $\bar{x}$ & 1.38 & & & \\
\hline $09: 18$ AM & $x$ & 1.40 & & & \\
\hline $09: 50$ AM & $x$ & 1.36 & 41 & & \\
\hline $10: 42 \mathrm{AM}$ & $x$ & 1.38 & & & \\
\hline 11:18 AM & $X$ & 1.37 & & & \\
\hline 01:00 PM & $x$ & 1.36 & & & \\
\hline $01: 38$ PM & $\bar{x}$ & 1.29 & 39 & & \\
\hline $01: 50 \mathrm{PM}$ & $x$ & 1.39 & 47 & & \\
\hline $02: 26$ PM & $x$ & 1.38 & & & \\
\hline $02: 47 \mathrm{PM}$ & $\bar{x}$ & 1.35 & 43 & & \\
\hline 03:00 PM & $E$ & 1.45 & & & \\
\hline 03:15 PM & $F$ & 1.54 & & & \\
\hline $03: 38$ PM & $F$ & 1.53 & 96 & & \\
\hline 03:54 PM & $F$ & 1.58 & & & \\
\hline 04:16 PM & $\mathrm{F}$ & 1.53 & & & \\
\hline 04:38 PM & $F$ & 1.54 & 112 & & \\
\hline & & & & & \\
\hline & & & & & \\
\hline & & & & & \\
\hline & & & & & \\
\hline & & & & & \\
\hline & & & & & \\
\hline & & & & & \\
\hline & & & & & \\
\hline & & & & & \\
\hline
\end{tabular}




\section{ACT REMBCO JOINT VENTURE \\ Ornl Wag 4 Seeps 4 \& 6 Removal Action Project \\ Quality Contol Report \\ August 27, 1996}

\begin{tabular}{|c|c|c|c|c|c|}
\hline Time of Test & $\begin{array}{c}\text { Mix } \\
\text { Identificaton }\end{array}$ & $\begin{array}{c}\text { Specific } \\
\text { Gravity } \\
\text { (Kg/Litre) }\end{array}$ & $\begin{array}{c}\text { Marsh } \\
\text { Funnel } \\
\text { (seconds) }\end{array}$ & $\begin{array}{c}\text { Coefficient } \\
\text { Pressure } \\
\text { Filtration } \\
\text { (seconds-1/2) }\end{array}$ & $\begin{array}{c}\text { Static } \\
\text { Cone } \\
\text { Penetrometer } \\
\text { (mm) }\end{array}$ \\
\hline $08: 00 \mathrm{AM}$ & $\mathrm{X}$ & 1.39 & 48 & & \\
\hline $08: 43 \mathrm{AM}$ & $\mathrm{X}$ & 1.36 & & & \\
\hline $09: 15 \mathrm{AM}$ & $\mathrm{E}$ & 1.45 & 56 & & \\
\hline $09: 48 \mathrm{AM}$ & $\mathrm{E}$ & 1.43 & & & \\
\hline $10: 22 \mathrm{AM}$ & $\mathrm{F}$ & 1.55 & & & \\
\hline $10: 46 \mathrm{AM}$ & $\mathrm{F}$ & 1.57 & 116 & & \\
\hline $10: 59 \mathrm{AM}$ & $\mathrm{F}$ & 1.54 & & & \\
\hline $11: 07 \mathrm{AM}$ & $\mathrm{F}$ & 1.52 & 91 & & \\
\hline $11: 38 \mathrm{AM}$ & $\mathrm{F}$ & 1.55 & & & \\
\hline $01: 16 \mathrm{PM}$ & $\mathrm{F}$ & 1.54 & & & \\
\hline $01: 41 \mathrm{PM}$ & $\mathrm{F}$ & 1.59 & & & \\
\hline $02: 02 \mathrm{PM}$ & $\mathrm{F}$ & 1.58 & Infinite & & \\
\hline $02: 22 \mathrm{PM}$ & $\mathrm{F}$ & 1.55 & & & \\
\hline $02: 52 \mathrm{PM}$ & $\mathrm{F}$ & 1.54 & & & \\
\hline $03: 11 \mathrm{PM}$ & $\mathrm{F}$ & 1.54 & & & \\
\hline $03: 28 \mathrm{PM}$ & $\mathrm{F}$ & 1.54 & & & \\
\hline $03: 49 \mathrm{PM}$ & $\mathrm{F}$ & 1.57 & 88 & & \\
\hline $04: 01 \mathrm{PM}$ & $\mathrm{F}$ & 1.53 & & & \\
\hline $04: 10 \mathrm{PM}$ & $\mathrm{F}$ & 1.53 & & & \\
\hline & & & & & \\
\hline & & & & & \\
\hline & & & & & \\
\hline & & & & & \\
\hline & & & & & \\
\hline
\end{tabular}

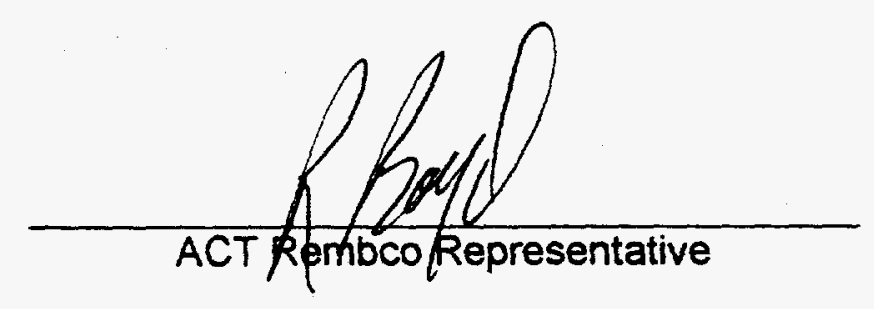




\section{ACT REMBCO JOINT VENTURE \\ Ornl Wag 4 Seeps 4 \& 6 Removal Action Project}

Quality Contol Report

August 28, 1996

\begin{tabular}{|c|c|c|c|c|c|}
\hline Time of Test & $\begin{array}{c}\text { Mix } \\
\text { Identificaton }\end{array}$ & $\begin{array}{c}\text { Specific } \\
\text { Gravity } \\
\text { (Kg/Litre) }\end{array}$ & $\begin{array}{c}\text { Marsh } \\
\text { Funnel } \\
\text { (seconds) }\end{array}$ & $\begin{array}{c}\text { Coefficient } \\
\text { Pressure } \\
\text { Filtration } \\
\text { (seconds-1/2) }\end{array}$ & $\begin{array}{c}\text { Static } \\
\text { Cone } \\
\text { Penetrometer } \\
\text { (mm) }\end{array}$ \\
\hline $08: 10 \mathrm{AM}$ & $\mathrm{M}$ & 1.26 & 47 & & \\
\hline $08: 26 \mathrm{AM}$ & $\mathrm{M}$ & 1.27 & & & \\
\hline $08: 56 \mathrm{AM}$ & $\mathrm{M}$ & 1.25 & & & \\
\hline $09: 14 \mathrm{AM}$ & $\mathrm{M}$ & 1.26 & & & \\
\hline $09: 37 \mathrm{AM}$ & $\mathrm{M}$ & 1.23 & 44 & & \\
\hline $10: 00 \mathrm{AM}$ & $\mathrm{X}$ & 1.38 & & & \\
\hline $10: 36 \mathrm{AM}$ & $\mathrm{X}$ & 1.36 & 46 & & \\
\hline $11: 14 \mathrm{AM}$ & $\mathrm{E}$ & 1.46 & & & \\
\hline $11: 45 \mathrm{AM}$ & $\mathrm{F}$ & 1.55 & & & \\
\hline $01: 07 \mathrm{PM}$ & $\mathrm{F}$ & 1.57 & & & \\
\hline $01: 31 \mathrm{PM}$ & $\mathrm{F}$ & 1.53 & 91 & & \\
\hline $01: 53 \mathrm{PM}$ & $\mathrm{F}$ & 1.54 & & & \\
\hline $02: 18 \mathrm{PM}$ & $\mathrm{F}$ & 1.55 & 112 & & \\
\hline $02: 49 \mathrm{PM}$ & $\mathrm{F}$ & 1.54 & & & \\
\hline $03: 06 \mathrm{PM}$ & $\mathrm{F}$ & 1.53 & Infinite & & \\
\hline $03: 23 \mathrm{PM}$ & $\mathrm{F}$ & 1.55 & & & \\
\hline $03: 44 \mathrm{PM}$ & $\mathrm{F}$ & 1.58 & & & \\
\hline $04: 00 \mathrm{PM}$ & $\mathrm{F}$ & 1.57 & 88 & & \\
\hline & & & & & \\
\hline & & & & & \\
\hline & & & & & \\
\hline & & & & & \\
\hline & & & & & \\
\hline & & & & & \\
\hline
\end{tabular}

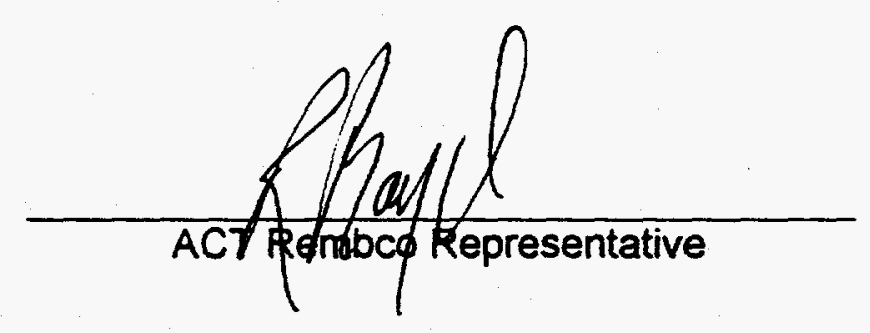




\section{ACT REMBCO JOINT VENTURE \\ Ornl Wag 4 Seeps 4 \& 6 Removal Action Project}

Quality Contol Report

August 29, 1996

\begin{tabular}{|c|c|c|c|c|c|}
\hline Time of Test & $\begin{array}{c}\text { Mix } \\
\text { Identificaton }\end{array}$ & $\begin{array}{c}\text { Specific } \\
\text { Gravity } \\
\text { (Kg/Litre) }\end{array}$ & $\begin{array}{c}\text { Marsh } \\
\text { Funnel } \\
\text { (seconds) }\end{array}$ & $\begin{array}{c}\text { Coefficient } \\
\text { Pressure } \\
\text { Filtration } \\
\text { (seconds-1/2) }\end{array}$ & $\begin{array}{c}\text { Static } \\
\text { Cone } \\
\text { Penetrometer } \\
\text { (mm) }\end{array}$ \\
\hline $07: 40 \mathrm{AM}$ & $\mathrm{X}$ & 1.37 & & & \\
\hline $07: 47 \mathrm{AM}$ & $\mathrm{X}$ & 1.36 & 44 & & \\
\hline $07: 54 \mathrm{AM}$ & $\mathrm{X}$ & 1.37 & & & \\
\hline $08: 10 \mathrm{AM}$ & $\mathrm{E}$ & 1.45 & 60 & & \\
\hline $08: 15 \mathrm{AM}$ & $\mathrm{E}$ & 1.39 & & & \\
\hline $08: 21 \mathrm{AM}$ & $\mathrm{E}$ & 1.43 & 55 & & \\
\hline $09: 03 \mathrm{AM}$ & $\mathrm{F}$ & 1.53 & 87 & & \\
\hline $09: 15 \mathrm{AM}$ & $\mathrm{F}$ & 1.54 & & & \\
\hline $09: 29 \mathrm{AM}$ & $\mathrm{F}$ & 1.55 & & & \\
\hline $09: 47 \mathrm{AM}$ & $\mathrm{F}$ & 1.57 & 132 & & \\
\hline $10: 02 \mathrm{AM}$ & $\mathrm{F}$ & 1.55 & & & \\
\hline $10: 17 \mathrm{AM}$ & $\mathrm{F}$ & 1.54 & & & \\
\hline $10: 34 \mathrm{AM}$ & $\mathrm{F}$ & 1.53 & 106 & & \\
\hline $10: 48 \mathrm{AM}$ & $\mathrm{F}$ & 1.55 & & & \\
\hline $11: 17 \mathrm{AM}$ & $\mathrm{F}$ & 1.54 & & & \\
\hline $11: 22 \mathrm{AM}$ & $\mathrm{F}$ & 1.53 & 96 & & \\
\hline $01: 10 \mathrm{PM}$ & $\mathrm{X}$ & 1.38 & & & \\
\hline $01: 30 \mathrm{PM}$ & $\mathrm{X}$ & 1.36 & 44 & & \\
\hline $01: 42 \mathrm{PM}$ & $\mathrm{X}$ & 1.38 & & & \\
\hline $02: 03 \mathrm{PM}$ & $\mathrm{E}$ & 1.44 & & & \\
\hline $02: 15 \mathrm{PM}$ & $\mathrm{E}$ & 1.44 & & & \\
\hline $02: 23 \mathrm{PM}$ & $\mathrm{E}$ & 1.43 & 59 & & \\
\hline $02: 29 \mathrm{PM}$ & $\mathrm{E}$ & 1.44 & & & \\
\hline & & & & & \\
\hline & & & & & \\
\hline & & & & & \\
\hline & & & & & \\
\hline & & & & & \\
\hline & & & & & \\
\hline & & & & & \\
\hline & & & & & \\
\hline & & & & & \\
\hline & & & & & \\
\hline & & & & & \\
\hline & & & & & \\
\hline & & & & & \\
\hline & & & & & \\
\hline & & & & \\
\hline
\end{tabular}




\section{ACT REMBCO JOINT VENTURE \\ Ornl Wag 4 Seeps 4 \& 6 Removal Action Project}

Quality Contol Report

August 30, 1996

\begin{tabular}{|c|c|c|c|c|c|}
\hline Time of Test & $\begin{array}{c}\text { Mix } \\
\text { Identificaton }\end{array}$ & $\begin{array}{c}\text { Specific } \\
\text { Gravity } \\
\text { (Kg/Litre) }\end{array}$ & $\begin{array}{c}\text { Marsh } \\
\text { Funnel } \\
\text { (seconds) }\end{array}$ & $\begin{array}{l}\text { Coefficient } \\
\text { Pressure } \\
\text { Filtration } \\
\text { (seconds-1/2) }\end{array}$ & $\begin{array}{c}\text { Static } \\
\text { Cone } \\
\text { Penetrometer } \\
(\mathrm{mm})\end{array}$ \\
\hline $07: 40 \mathrm{AM}$ & $\bar{x}$ & 1.32 & 42 & & \\
\hline $07: 50 \mathrm{AM}$ & $\bar{x}$ & 1.36 & 45 & & \\
\hline $08: 04 \mathrm{AM}$ & $\bar{x}$ & 1.38 & 47 & & \\
\hline $08: 22 \mathrm{AM}$ & $\bar{x}$ & 1.38 & 47 & & \\
\hline $08: 34 \mathrm{AM}$ & $\bar{E}$ & 1.44 & & & \\
\hline $08: 42 \mathrm{AM}$ & $E$ & 1.45 & 59 & & \\
\hline $08: 53 \mathrm{AM}$ & $E$ & 1.44 & & & \\
\hline$\overline{09: 10 \mathrm{AM}}$ & $\bar{F}$ & 1.55 & & & \\
\hline $09: 18 \mathrm{AM}$ & $F$ & 1.57 & & & \\
\hline $09: 28 \mathrm{AM}$ & $\bar{F}$ & 1.54 & 76 & & \\
\hline $09: 35 \mathrm{AM}$ & $\bar{F}$ & 1.54 & & & \\
\hline $09: 57 \mathrm{AM}$ & $F$ & 1.54 & 92 & & \\
\hline $10: 04 \mathrm{AM}$ & $F$ & 1.59 & & & \\
\hline $10: 33 \mathrm{AM}$ & $F$ & 1.53 & & & \\
\hline $11: 06$ AM & $F$ & 1.53 & 112 & & \\
\hline $11: 22 \mathrm{AM}$ & $F$ & 1.53 & & & \\
\hline $11: 39 \mathrm{AM}$ & $F$ & 1.53 & & & \\
\hline $01: 00$ PM & $F$ & 1.54 & 81 & & \\
\hline $01: 18 \mathrm{PM}$ & $\bar{F}$ & 1.53 & & & \\
\hline $01: 41 \mathrm{PM}$ & $F$ & 1.53 & & & \\
\hline $01: 52 \mathrm{PM}$ & $F$ & 1.56 & 96 & & \\
\hline 02:02 PM & $F$ & 1.52 & & & \\
\hline & & & & & \\
\hline & & & & & \\
\hline & & & & & \\
\hline & & & & & \\
\hline & & & & & \\
\hline & & & & & \\
\hline & & & & & \\
\hline
\end{tabular}




\section{ACT REMBCO JOINT VENTURE \\ Ornl Wag 4 Seeps 4 \& 6 Removal Action Project}

Quality Contol Report

September 03, 1996

\begin{tabular}{|c|c|c|c|c|c|}
\hline Time of Test & $\begin{array}{c}\text { Mix } \\
\text { Identificaton }\end{array}$ & $\begin{array}{c}\text { Specific } \\
\text { Gravity } \\
\text { (Kg/Litre) }\end{array}$ & $\begin{array}{c}\text { Marsh } \\
\text { Funnel } \\
\text { (seconds) }\end{array}$ & $\begin{array}{l}\text { Coefficient } \\
\text { Pressure } \\
\text { Filtration } \\
\text { (seconds-1/2) }\end{array}$ & $\begin{array}{c}\text { Static } \\
\text { Cone } \\
\text { Penetrometer } \\
(\mathrm{mm}) \\
\end{array}$ \\
\hline $07: 58 \mathrm{AM}$ & $x$ & 1.33 & & & \\
\hline $08: 12 \mathrm{AM}$ & $\bar{x}$ & 1.37 & 44 & & \\
\hline $08: 21 \mathrm{AM}$ & $\bar{x}$ & 1.38 & & & \\
\hline $08: 27$ AM & $\bar{x}$ & 1.38 & 46 & & \\
\hline $08: 32 \mathrm{AM}$ & $\bar{x}$ & 1.35 & & & \\
\hline $08: 58 \mathrm{AM}$ & $E$ & 1.44 & 59 & & \\
\hline $09: 12 \mathrm{AM}$ & $\bar{E}$ & 1.44 & & & \\
\hline $09: 21 \mathrm{AM}$ & $E$ & 1.44 & & & \\
\hline $09: 35 \mathrm{AM}$ & $E$ & 1.42 & & & \\
\hline $09: 42 \mathrm{AM}$ & $E$ & 1.45 & 56 & & \\
\hline $09: 56 \mathrm{AM}$ & $E$ & 1.45 & & & \\
\hline $10: 09 \mathrm{AM}$ & $E$ & 1.44 & 58 & & \\
\hline $10: 16 \mathrm{AM}$ & $E$ & 1.45 & & & \\
\hline $10: 24 \mathrm{AM}$ & $E$ & 1.45 & & & \\
\hline $10: 37 \mathrm{AM}$ & $E$ & 1.45 & & & \\
\hline $10: 53 \mathrm{AM}$ & $E$ & 1.45 & & & \\
\hline 11:03 AM & $E$ & 1.47 & 71 & & \\
\hline $11: 16 \mathrm{AM}$ & $E$ & 1.46 & & & \\
\hline $11: 30 \mathrm{AM}$ & $E$ & 1.42 & 52 & & \\
\hline $11: 46 \mathrm{AM}$ & $E$ & 1.44 & & & \\
\hline $12: 52 \mathrm{PM}$ & $E$ & 1.45 & & & \\
\hline $01: 18 \mathrm{PM}$ & $E$ & 1.44 & & & \\
\hline $01: 33 P M$ & $E$ & 1.45 & 57 & & \\
\hline 01:42 PM & $E$ & 1.45 & & & \\
\hline $01: 51 \mathrm{PM}$ & $\bar{E}$ & 1.45 & 57 & & \\
\hline $02: 04 \mathrm{PM}$ & $E$ & 1.42 & & & \\
\hline $02: 16 P M$ & $E$ & 1.44 & 60 & & \\
\hline 02:22 PM & $E$ & 1.44 & & & \\
\hline $02: 30 P M$ & $\bar{E}$ & 1.44 & & & \\
\hline $02: 37 \mathrm{PM}$ & $E$ & 1.45 & & & \\
\hline 02:49PM & $E$ & 1.43 & 66 & & \\
\hline $02: 55 \mathrm{PM}$ & $E$ & 1.45 & & & \\
\hline 03:01 PM & $\bar{E}$ & 1.44 & & & \\
\hline
\end{tabular}




\section{ACT REMBCO JOINT VENTURE \\ Ornl Wag 4 Seeps 4 \& 6 Removal Action Project \\ Quality Contol Report \\ September 03, 1996}

\begin{tabular}{|c|c|c|c|c|c|}
\hline Time of Test & $\begin{array}{c}\text { Mix } \\
\text { Identificaton }\end{array}$ & $\begin{array}{c}\text { Specific } \\
\text { Gravity } \\
\text { (Kg/Litre) }\end{array}$ & $\begin{array}{c}\text { Marsh } \\
\text { Funnel } \\
\text { (seconds) }\end{array}$ & $\begin{array}{c}\text { Coefficient } \\
\text { Pressure } \\
\text { Filtration } \\
\text { (seconds-1/2) }\end{array}$ & $\begin{array}{c}\text { Static } \\
\text { Cone } \\
\text { Penetrometer } \\
\text { (mm) }\end{array}$ \\
\hline $03: 13 \mathrm{PM}$ & $\mathrm{E}$ & 1.45 & & & \\
\hline $03: 22 \mathrm{PM}$ & $\mathrm{E}$ & 1.45 & & & \\
\hline $03: 33 \mathrm{PM}$ & $\mathrm{E}$ & 1.45 & 64 & & \\
\hline $03: 40 \mathrm{PM}$ & $\mathrm{E}$ & 1.48 & & & \\
\hline $03: 52 \mathrm{PM}$ & $\mathrm{E}$ & 1.45 & & & \\
\hline & & & & & \\
\hline & & & & & \\
\hline
\end{tabular}

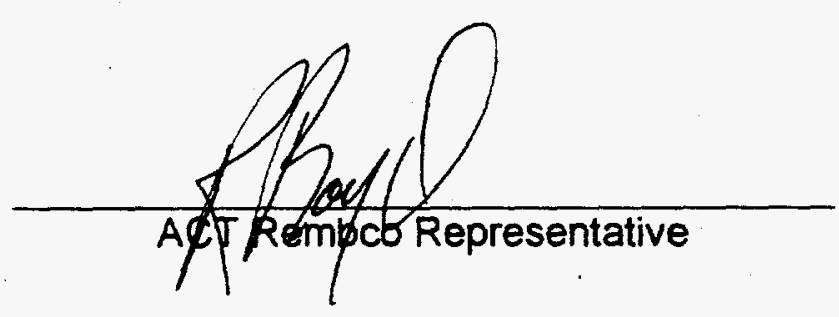




\section{ACT REMBCO JOINT VENTURE Ornl Wag 4 Seeps 4 \& 6 Removal Action Project \\ Quality Contol Report September 04, 1996}

\begin{tabular}{|c|c|c|c|c|c|}
\hline Time of Test & $\begin{array}{c}\text { Mix } \\
\text { Identificaton }\end{array}$ & $\begin{array}{c}\text { Specific } \\
\text { Gravity } \\
\text { (Kg/Litre) }\end{array}$ & $\begin{array}{c}\text { Marsh } \\
\text { Funnel } \\
\text { (seconds) }\end{array}$ & $\begin{array}{c}\text { Coefficient } \\
\text { Pressure } \\
\text { Filtration } \\
\text { (seconds-1/2) }\end{array}$ & $\begin{array}{c}\text { Static } \\
\text { Cone } \\
\text { Penetrometer } \\
\text { (mm) }\end{array}$ \\
\hline $08: 10 \mathrm{AM}$ & $\mathrm{X}$ & 1.38 & & & \\
\hline $08: 19 \mathrm{AM}$ & $\mathrm{X}$ & 1.38 & 45 & & \\
\hline $08: 28 \mathrm{AM}$ & $\mathrm{X}$ & 1.38 & & & \\
\hline $08: 33 \mathrm{AM}$ & $\mathrm{X}$ & 1.38 & & & \\
\hline $08: 55 \mathrm{AM}$ & $\mathrm{X}$ & 1.38 & 47 & & \\
\hline $09: 04 \mathrm{AM}$ & $\mathrm{X}$ & 1.38 & & & \\
\hline $09: 12 \mathrm{AM}$ & $\mathrm{X}$ & 1.40 & 50 & & \\
\hline $09: 22 \mathrm{AM}$ & $\mathrm{X}$ & 1.39 & 46 & & \\
\hline $09: 33 \mathrm{AM}$ & $\mathrm{X}$ & 1.38 & & & \\
\hline $09: 40 \mathrm{AM}$ & $\mathrm{X}$ & 1.38 & & & \\
\hline $09: 52 \mathrm{AM}$ & $\mathrm{E}$ & 1.44 & 58 & & \\
\hline $10: 10 \mathrm{AM}$ & $\mathrm{E}$ & 1.45 & & & \\
\hline $10: 17 \mathrm{AM}$ & $\mathrm{E}$ & 1.45 & & & \\
\hline $10: 22 \mathrm{AM}$ & $\mathrm{E}$ & 1.44 & & & \\
\hline $10: 33 \mathrm{AM}$ & $\mathrm{E}$ & 1.45 & 57 & & \\
\hline $10: 45 \mathrm{AM}$ & $\mathrm{E}$ & 1.44 & & & \\
\hline $11: 15 \mathrm{AM}$ & $\mathrm{E}$ & 1.45 & & & \\
\hline $11: 31 \mathrm{AM}$ & $\mathrm{E}$ & 1.50 & 65 & & \\
\hline $12: 49 \mathrm{PM}$ & $\mathrm{E}$ & 1.50 & & & \\
\hline $01: 07 \mathrm{PM}$ & $\mathrm{E}$ & 1.45 & & & \\
\hline $01: 20 \mathrm{PM}$ & $\mathrm{E}$ & 1.45 & & & \\
\hline $01: 58 \mathrm{PM}$ & $\mathrm{M}$ & 1.27 & 46 & & \\
\hline $02: 22 \mathrm{PM}$ & $\mathrm{M}$ & 1.22 & & & \\
\hline $02: 41 \mathrm{PM}$ & $\mathrm{M}$ & 1.26 & & & \\
\hline $03: 10 \mathrm{PM}$ & $\mathrm{M}$ & 1.26 & 46 & & \\
\hline $03: 37 \mathrm{PM}$ & $\mathrm{M}$ & 1.25 & & & \\
\hline & & & & & \\
\hline & & & & & \\
\hline & & & & \\
\hline
\end{tabular}




\section{ACT REMBCO JOINT VENTURE \\ Ornl Wag 4 Seeps 4 \& 6 Removal Action Project}

Quality Contol Report

September 05, 1996

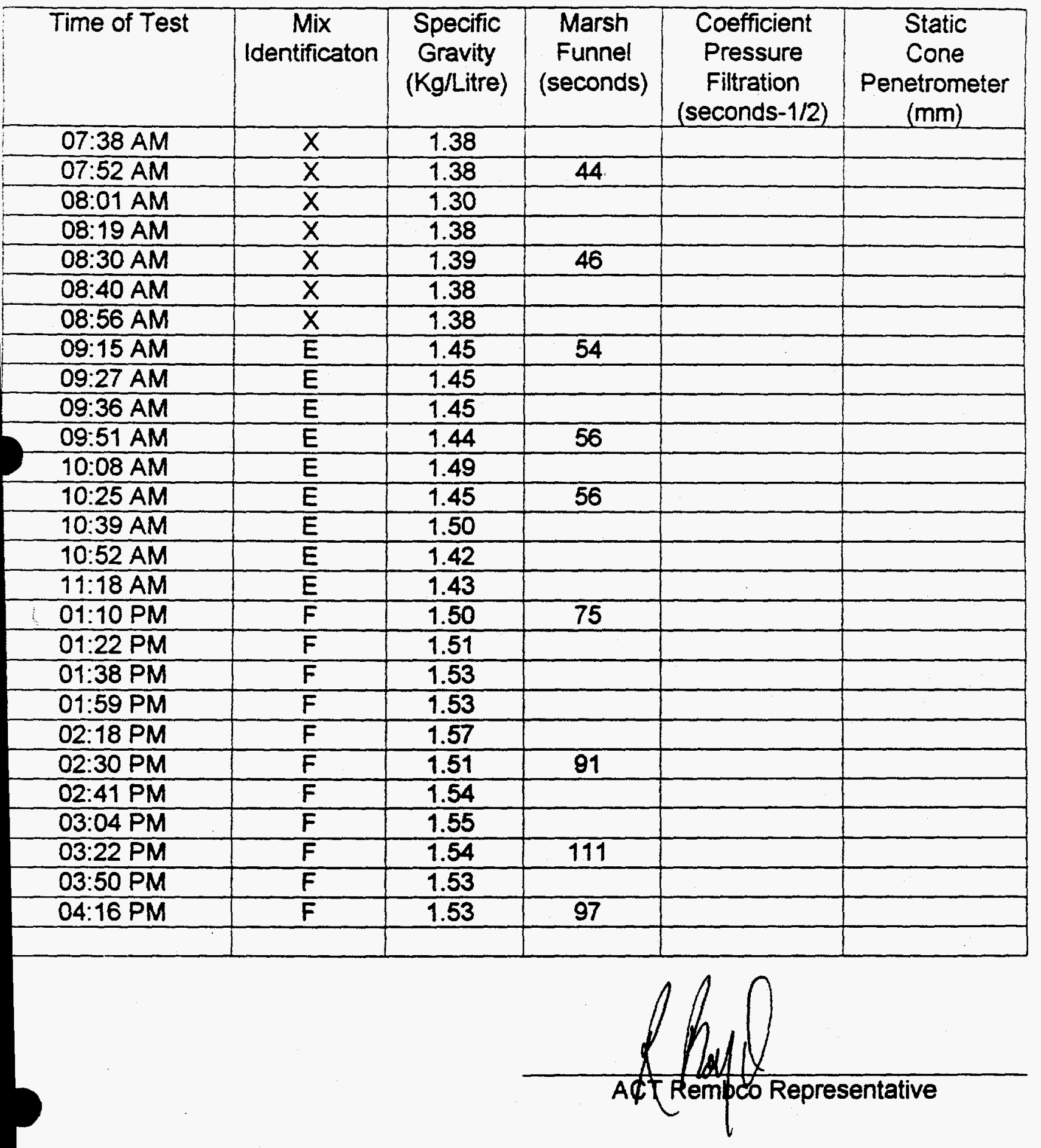




\section{ACT REMBCO JOINT VENTURE \\ Ornl Wag 4 Seeps 4 \& 6 Removal Action Project}

Quality Contol Report

September 09, 1996

\begin{tabular}{|c|c|c|c|c|c|}
\hline Time of Test & $\begin{array}{c}\text { Mix } \\
\text { Identificaton }\end{array}$ & $\begin{array}{c}\text { Specific } \\
\text { Gravity } \\
\text { (Kg/Litre) }\end{array}$ & $\begin{array}{c}\text { Marsh } \\
\text { Funnel } \\
\text { (seconds) }\end{array}$ & $\begin{array}{c}\text { Coefficient } \\
\text { Pressure } \\
\text { Filtration } \\
\text { (seconds-1/2) }\end{array}$ & $\begin{array}{c}\text { Static } \\
\text { Cone } \\
\text { Penetrometer } \\
\text { (mm) }\end{array}$ \\
\hline $09: 13 \mathrm{AM}$ & $\mathrm{X}$ & 1.38 & & & \\
\hline $09: 32 \mathrm{AM}$ & $\mathrm{X}$ & 1.35 & 45 & & \\
\hline $09: 44 \mathrm{AM}$ & $\mathrm{X}$ & 1.34 & & & \\
\hline $10: 12 \mathrm{AM}$ & $\mathrm{X}$ & 1.33 & 42 & & \\
\hline $10: 26 \mathrm{AM}$ & $\mathrm{X}$ & 1.37 & & & \\
\hline $10: 35 \mathrm{AM}$ & $\mathrm{X}$ & 1.38 & 48 & & \\
\hline $10: 51 \mathrm{AM}$ & $\mathrm{X}$ & 1.38 & 46 & & \\
\hline $11: 02 \mathrm{AM}$ & $\mathrm{X}$ & 1.40 & & & \\
\hline $11: 17 \mathrm{AM}$ & $\mathrm{X}$ & 1.39 & & & \\
\hline $11: 25 \mathrm{AM}$ & $\mathrm{X}$ & 1.38 & & & \\
\hline $11: 32 \mathrm{AM}$ & $\mathrm{X}$ & 1.38 & & & \\
\hline $01: 10 \mathrm{PM}$ & $\mathrm{X}$ & 1.38 & 47 & & \\
\hline $01: 23 \mathrm{PM}$ & $\mathrm{X}$ & 1.38 & & & \\
\hline $01: 48 \mathrm{PM}$ & $\mathrm{X}$ & 1.36 & 44 & & \\
\hline $02: 15 \mathrm{PM}$ & $\mathrm{X}$ & 1.38 & & & \\
\hline $02: 52 \mathrm{PM}$ & $\mathrm{E}$ & 1.45 & & & \\
\hline $03: 05 \mathrm{PM}$ & $\mathrm{E}$ & 1.44 & 54 & & \\
\hline $03: 22 \mathrm{PM}$ & $\mathrm{E}$ & 1.52 & & & \\
\hline $03: 40 \mathrm{PM}$ & $\mathrm{E}$ & 1.49 & 61 & & \\
\hline $03: 56 \mathrm{PM}$ & $\mathrm{E}$ & 1.44 & & & \\
\hline & & & & & \\
\hline & & & & & \\
\hline
\end{tabular}




\section{ACT REMBCO JOINT VENTURE \\ Ornl Wag 4 Seeps 4 \& 6 Removal Action Project}

Quality Contol Report

September 10, 1996

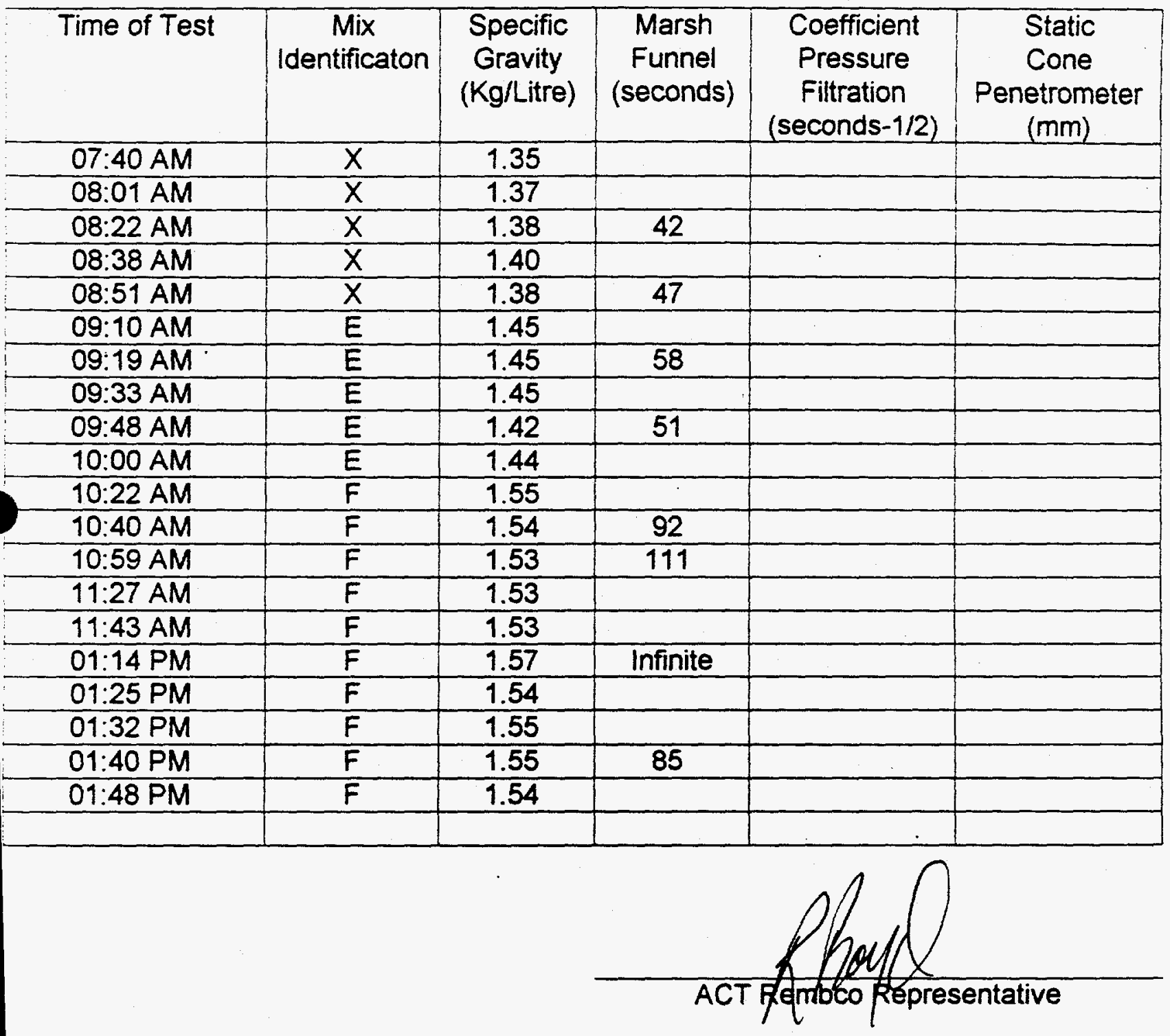




\section{ACT REMBCO JOINT VENTURE \\ Ornl Wag 4 Seeps 4 \& 6 Removal Action Project}

Quality Contol Report

September 11, 1996

\begin{tabular}{|c|c|c|c|c|c|}
\hline Time of Test & $\begin{array}{c}\text { Mix } \\
\text { Identificaton }\end{array}$ & $\begin{array}{c}\text { Specific } \\
\text { Gravity } \\
\text { (Kg/Litre) }\end{array}$ & $\begin{array}{c}\text { Marsh } \\
\text { Funnel } \\
\text { (seconds) }\end{array}$ & $\begin{array}{l}\text { Coefficient } \\
\text { Pressure } \\
\text { Filtration } \\
\text { (seconds-1/2) }\end{array}$ & $\begin{array}{c}\text { Static } \\
\text { Cone } \\
\text { Penetrometer } \\
(\mathrm{mm})\end{array}$ \\
\hline $07: 45 \mathrm{AM}$ & $E$ & 1.45 & 56 & & \\
\hline $08: 22 \mathrm{AM}$ & $E$ & 1.45 & & & \\
\hline $08: 39 \mathrm{AM}$ & $E$ & 1.42 & 53 & & \\
\hline 09:01 AM & $E$ & 1.44 & & & \\
\hline $09: 26 \mathrm{AM}$ & $E$ & 1.45 & 57 & & \\
\hline $09: 36 \mathrm{AM}$ & $E$ & 1.45 & & & \\
\hline$\overline{09: 51 \mathrm{AM}}$ & $E$ & 1.45 & 58 & & \\
\hline $10: 15 \mathrm{AM}$ & $E$ & 1.45 & & & \\
\hline $10: 33 \mathrm{AM}$ & $E$ & 1.49 & & & \\
\hline $10: 58 \mathrm{AM}$ & $E$ & 1.44 & & & \\
\hline $11: 12 \mathrm{AM}$ & $E$ & 1.44 & & & \\
\hline $11: 25 \mathrm{AM}$ & $E$ & 1.45 & & & \\
\hline $11: 37 \mathrm{AM}$ & $E$ & 1.45 & 60 & & \\
\hline $01: 21 \mathrm{PM}$ & $E$ & 1.45 & & & \\
\hline $01: 30 \mathrm{PM}$ & $E$ & 1.45 & & & \\
\hline $01: 44 \mathrm{PM}$ & $E$ & 1.44 & & & \\
\hline $02: 08 \mathrm{PM}$ & $E$ & 1.41 & & & \\
\hline $02: 30 \mathrm{PM}$ & $E$ & 1.47 & 55 & & \\
\hline $02: 52 \mathrm{PM}$ & $E$ & 1.45 & & & \\
\hline $03: 45 \mathrm{PM}$ & $\bar{F}$ & 1.54 & & & \\
\hline 03:52 PM & $F$ & 1.54 & 107 & & \\
\hline & & & & & \\
\hline & & & & 11 & \\
\hline
\end{tabular}




\section{ACT REMBCO JOINT VENTURE \\ Ornl Wag 4 Seeps 4 \& 6 Removal Action Project}

Quality Contol Report

September 12, 1996

\begin{tabular}{|c|c|c|c|c|c|}
\hline Time of Test & $\begin{array}{c}\text { Mix } \\
\text { Identificaton }\end{array}$ & $\begin{array}{c}\text { Specific } \\
\text { Gravity } \\
\text { (Kg/Litre) }\end{array}$ & $\begin{array}{c}\text { Marsh } \\
\text { Funnel } \\
\text { (seconds) }\end{array}$ & $\begin{array}{l}\text { Coefficient } \\
\text { Pressure } \\
\text { Filtration } \\
\text { (seconds-1/2) }\end{array}$ & $\begin{array}{c}\text { Static } \\
\text { Cone } \\
\text { Penetrometer } \\
(\mathrm{mm})\end{array}$ \\
\hline $07: 40 \mathrm{AM}$ & $\bar{M}$ & 1.27 & 48 & & \\
\hline $07: 50$ AM & $\bar{M}$ & 1.25 & & & \\
\hline $08: 03 \mathrm{AM}$ & $\bar{M}$ & 1.21 & 42 & & \\
\hline $08: 32 \mathrm{AM}$ & $\bar{M}$ & 1.25 & & & \\
\hline $08: 45 \mathrm{AM}$ & $\bar{M}$ & 1.28 & 45 & & \\
\hline $09: 05 \mathrm{AM}$ & $\bar{M}$ & 1.28 & & & \\
\hline $09: 26 \mathrm{AM}$ & $M$ & 1.27 & & & \\
\hline $09: 48 \mathrm{AM}$ & $\bar{M}$ & 1.30 & 46 & & \\
\hline $10: 18 \mathrm{AM}$ & $\bar{M}$ & 1.28 & & & \\
\hline $10: 42 \mathrm{AM}$ & $M^{\prime}$ & 1.32 & 56 & & \\
\hline $10: 57 \mathrm{AM}$ & $M^{\prime}$ & 1.33 & 59 & & \\
\hline $11: 05 \mathrm{AM}$ & $M^{\prime}$ & 1.31 & & & \\
\hline $11: 14 \mathrm{AM}$ & $M^{\prime}$ & 1.29 & & & \\
\hline $11: 22 \mathrm{AM}$ & $M^{\prime}$ & 1.31 & & & \\
\hline $01: 30 \mathrm{PM}$ & $M^{\prime}$ & 1.31 & & & \\
\hline 12:00 AM & $M^{\prime}$ & 1.32 & 59 & & \\
\hline $02: 00 \mathrm{PM}$ & $M^{\prime}$ & 1.31 & & & \\
\hline $02: 22 \mathrm{PM}$ & $M^{\prime}$ & 1.31 & & & \\
\hline $02: 45 \mathrm{PM}$ & $M^{\prime}$ & 1.30 & 55 & & \\
\hline $03: 00 \mathrm{PM}$ & $M^{\prime}$ & 1.31 & 57 & & \\
\hline $03: 25 \mathrm{PM}$ & $M^{\prime}$ & 1.32 & & & \\
\hline 03:50 PM & $M$. & 1.31 & 57 & & \\
\hline & & & & & \\
\hline & & & & 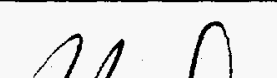 & \\
\hline
\end{tabular}




\section{ACT REMBCO JOINT VENTURE \\ Ornl Wag 4 Seeps 4 \& 6 Removal Action Project}

Quality Contol Report

September 13, 1996

\begin{tabular}{|c|c|c|c|c|c|}
\hline Time of Test & $\begin{array}{c}\text { Mix } \\
\text { Identificaton }\end{array}$ & $\begin{array}{c}\text { Specific } \\
\text { Gravity } \\
\text { (Kg/Litre) }\end{array}$ & $\begin{array}{c}\text { Marsh } \\
\text { Funnel } \\
\text { (seconds) }\end{array}$ & $\begin{array}{l}\text { Coefficient } \\
\text { Pressure } \\
\text { Filtration } \\
\text { (seconds-1/2) }\end{array}$ & $\begin{array}{c}\text { Static } \\
\text { Cone } \\
\text { Penetrometer } \\
(\mathrm{mm})\end{array}$ \\
\hline $07: 45 \mathrm{AM}$ & $M^{\prime}$ & 1.31 & 55 & & \\
\hline $08: 30 \mathrm{AM}$ & $M^{\prime}$ & 1.34 & 62 & & \\
\hline $08: 45$ AM & $\overline{M^{\prime}}$ & 1.30 & 52 & & \\
\hline $09: 40$ AM & $F$ & 1.53 & Infinite & & \\
\hline 10:00 AM & $F$ & 1.55 & 90 & & \\
\hline & & & & & \\
\hline & & & & & \\
\hline & & & & & \\
\hline & & & & & \\
\hline & & & & & \\
\hline & & & & & \\
\hline & & & & & \\
\hline & & & & & \\
\hline & & & & & \\
\hline
\end{tabular}

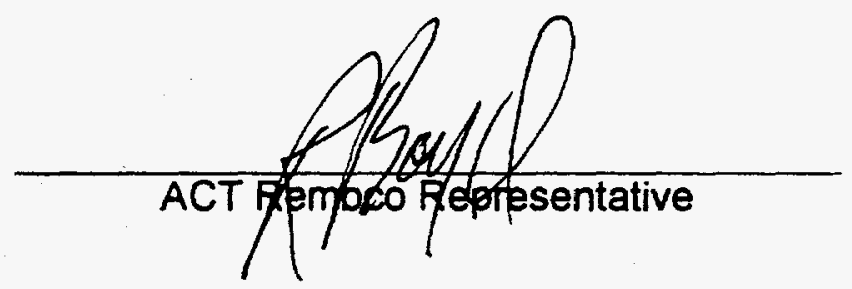




\section{ACT REMBCO JOINT VENTURE \\ Ornl Wag 4 Seeps 4 \& 6 Removal Action Project}

Quality Contol Report

September 17, 1996

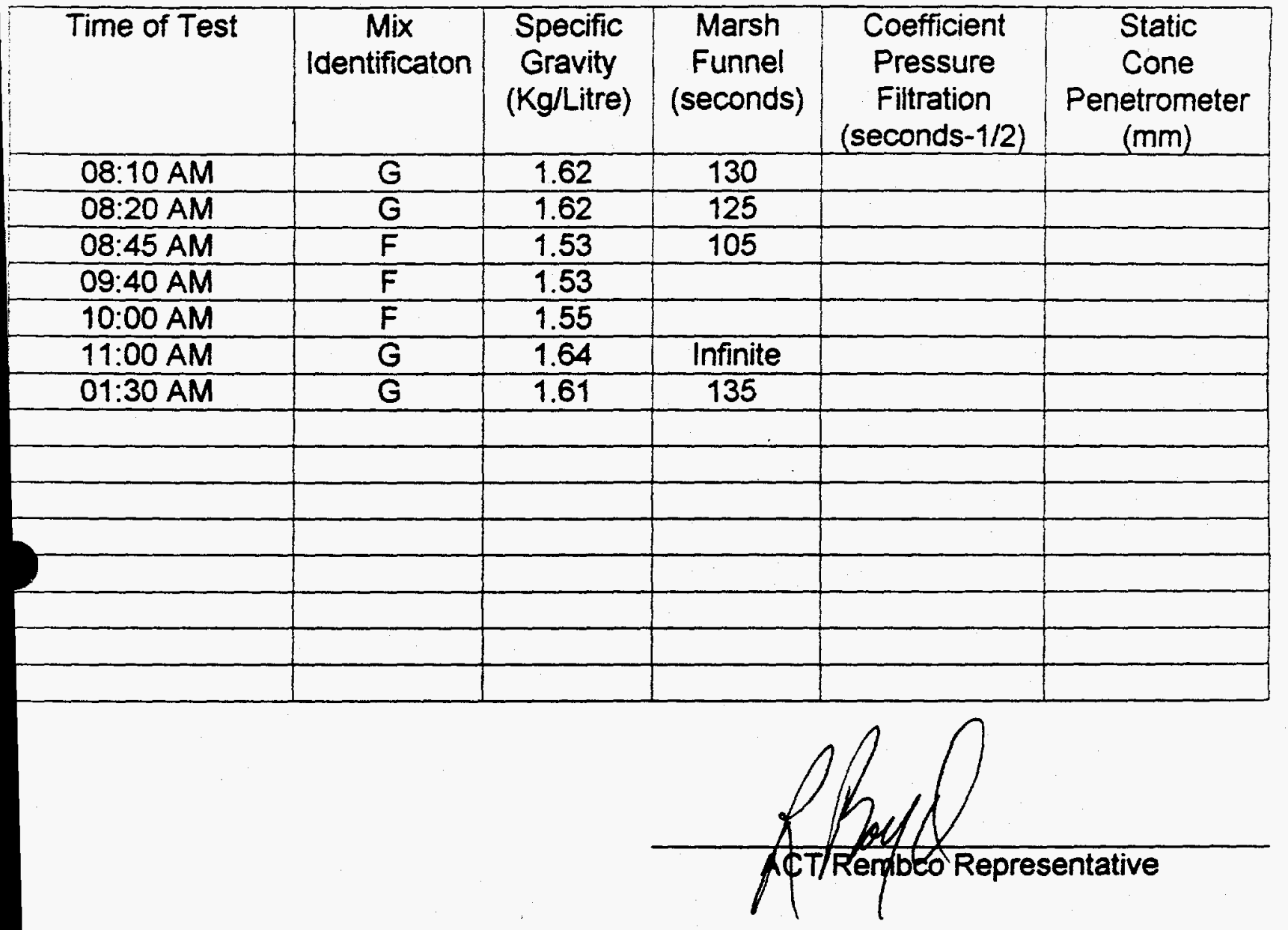




\section{ACT REMBCO JOINT VENTURE \\ Ornl Wag 4 Seeps 4 \& 6 Removal Action Project \\ Quality Contol Report \\ September 18, 1996}

\begin{tabular}{|c|c|c|c|c|c|}
\hline Time of Test & $\begin{array}{c}\text { Mix } \\
\text { Identificaton }\end{array}$ & $\begin{array}{c}\text { Specific } \\
\text { Gravity } \\
\text { (Kg/Litre) }\end{array}$ & $\begin{array}{c}\text { Marsh } \\
\text { Funnel } \\
\text { (seconds) }\end{array}$ & $\begin{array}{l}\text { Coefficient } \\
\text { Pressure } \\
\text { Filtration } \\
\text { (seconds-1/2) }\end{array}$ & $\begin{array}{c}\text { Static } \\
\text { Cone } \\
\text { Penetrometer } \\
(\mathrm{mm})\end{array}$ \\
\hline $08: 10 \mathrm{AM}$ & $F$ & 1.54 & 107 & & \\
\hline $08: 20 \mathrm{AM}$ & $F$ & 1.53 & & & \\
\hline $08: 45 \mathrm{AM}$ & $F$ & 1.53 & 92 & & \\
\hline $09: 13 \mathrm{AM}$ & $G^{\prime}$ & 1.59 & 120 & & \\
\hline 09:30 AM & $G^{\prime}$ & 1.58 & & & \\
\hline $09: 45 \mathrm{AM}$ & $G^{\prime}$ & 1.60 & & & \\
\hline $10: 00 \mathrm{AM}$ & $G^{\prime}$ & 1.55 & & & \\
\hline $10: 22 \mathrm{AM}$ & $G^{\prime}$ & 1.58 & Infinite & & \\
\hline $10: 46 \mathrm{AM}$ & $G^{\prime}$ & 1.58 & & & \\
\hline 10:59 AM & $G^{\prime}$ & 1.59 & Infinite & & \\
\hline $11: 16 \mathrm{AM}$ & $\mathrm{G}^{\prime}$ & 1.57 & & & \\
\hline $11: 29 \mathrm{AM}$ & $G^{\prime}$ & 1.54 & & & \\
\hline $01: 10 P M$ & $G^{\prime}$ & 1.58 & & & \\
\hline $01: 27 \mathrm{PM}$ & $G^{\prime}$ & 1.58 & Infinite & & \\
\hline $01: 45 \mathrm{PM}$ & $G^{\prime}$ & 1.59 & & & \\
\hline $02: 22 \mathrm{PM}$ & $G^{\prime}$ & 1.60 & & & \\
\hline 02:49 PM & $G^{\prime}$ & 1.58 & 140 & & \\
\hline $03: 10 \mathrm{PM}$ & $G^{\prime}$ & 1.58 & & & \\
\hline $03: 47 \mathrm{PM}$ & $G^{\prime}$ & 1.59 & & & \\
\hline $04: 12 \mathrm{PM}$ & $G^{\prime}$ & 1.61 & & & \\
\hline 04:30 PM & $G^{\prime}$ & 1.58 & 130 & & \\
\hline & & & & & \\
\hline & & & & & \\
\hline
\end{tabular}

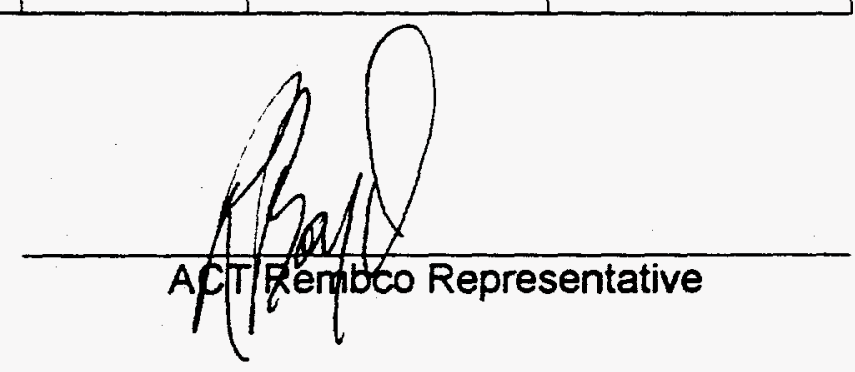




\section{ACT REMBCO JOINT VENTURE \\ Ornl Wag 4 Seeps 4 \& 6 Removal Action Project}

Quality Contol Report

September 19, 1996

\begin{tabular}{|c|c|c|c|c|c|}
\hline Time of Test & $\begin{array}{c}\text { Mix } \\
\text { Identificaton }\end{array}$ & $\begin{array}{c}\text { Specific } \\
\text { Gravity } \\
\text { (Kg/Litre) }\end{array}$ & $\begin{array}{c}\text { Marsh } \\
\text { Funnel } \\
\text { (seconds) }\end{array}$ & $\begin{array}{l}\text { Coefficient } \\
\text { Pressure } \\
\text { Filtration } \\
\text { (seconds- } 1 / 2 \text { ) }\end{array}$ & $\begin{array}{c}\text { Static } \\
\text { Cone } \\
\text { Penetrometer } \\
(\mathrm{mm})\end{array}$ \\
\hline $10: 22 \mathrm{AM}$ & $F$ & 1.53 & & & \\
\hline $10: 38 \mathrm{AM}$ & $\bar{F}$ & 1.53 & 92 & & \\
\hline $10: 52 \mathrm{AM}$ & $F$ & 1.55 & & & \\
\hline $11: 10 \mathrm{AM}$ & $\bar{F}$ & 1.58 & Infinite & & \\
\hline $11: 25 \mathrm{AM}$ & $F$ & 1.53 & & & \\
\hline $11: 37 \mathrm{AM}$ & $F$ & 1.53 & 122 & & \\
\hline $11: 48 \mathrm{AM}$ & $F$ & 1.54 & & & \\
\hline $01: 16 \mathrm{PM}$ & $\bar{F}$ & 1.54 & & & \\
\hline $01: 29 \mathrm{PM}$ & $\bar{F}$ & 1.53 & 110 & & \\
\hline $01: 42 \mathrm{PM}$ & $\bar{F}$ & 1.53 & & & \\
\hline $01: 59 \mathrm{PM}$ & $\bar{F}$ & 1.56 & Infinite & & \\
\hline $02: 12 \mathrm{PM}$ & $\bar{F}$ & 1.54 & & & \\
\hline 02:30 PM & $\bar{F}$ & 1.54 & 120 & & \\
\hline $02: 43 \mathrm{PM}$ & $F$ & 1.50 & & & \\
\hline $02: 58 \mathrm{PM}$ & $F$ & 1.51 & & & \\
\hline 03:07 PM & $\mathbf{F}$ & 1.53 & Infinite & & \\
\hline $03: 23 \mathrm{PM}$ & $F$ & 1.55 & & & \\
\hline $03: 37 \mathrm{PM}$ & $F$ & 1.53 & & & \\
\hline $03: 58 \mathrm{PM}$ & $\bar{F}$ & 1.51 & & & \\
\hline $04: 10 \mathrm{PM}$ & $F$ & 1.52 & & & \\
\hline \multirow[t]{3}{*}{$04: 30 \mathrm{PM}$} & $F$ & 1.55 & & & \\
\hline & & & & & \\
\hline & & & & & \\
\hline & & & & empoco kepr & ative \\
\hline
\end{tabular}




\section{ACT REMBCO JOINT VENTURE \\ Ornl Wag 4 Seeps 4 \& 6 Removal Action Project}

Quality Contol Report

September 20, 1996

\begin{tabular}{|c|c|c|c|c|c|}
\hline Time of Test & $\begin{array}{c}\text { Mix } \\
\text { Identificaton }\end{array}$ & $\begin{array}{c}\text { Specific } \\
\text { Gravity } \\
\text { (Kg/Litre) }\end{array}$ & $\begin{array}{c}\text { Marsh } \\
\text { Funnel } \\
\text { (seconds) }\end{array}$ & $\begin{array}{l}\text { Coefficient } \\
\text { Pressure } \\
\text { Filtration } \\
\text { (seconds-1/2) }\end{array}$ & $\begin{array}{c}\text { Static } \\
\text { Cone } \\
\text { Penetrometer } \\
(\mathrm{mm})\end{array}$ \\
\hline $08: 54 \mathrm{AM}$ & $F$ & 1.58 & Infinite & & \\
\hline $09: 23 \mathrm{AM}$ & $\bar{F}$ & 1.52 & & & \\
\hline $09: 45 \mathrm{AM}$ & $F$ & 1.54 & & & \\
\hline $10: 04 \mathrm{AM}$ & $\bar{F}$ & 1.55 & 123 & & \\
\hline $10: 32 \mathrm{AM}$ & $G^{\prime}$ & 1.59 & & & \\
\hline $10: 46 \mathrm{AM}$ & $\mathrm{G}^{\prime}$ & 1.58 & & & \\
\hline $11: 04 \mathrm{AM}$ & $G^{\prime}$ & 1.61 & Infinite & & \\
\hline $11: 22 \mathrm{AM}$ & $G^{\prime}$ & 1.56 & & & \\
\hline $11: 47 \mathrm{AM}$ & $G^{\prime}$ & 1.58 & & & \\
\hline $12: 52 \mathrm{PM}$ & $G^{\prime}$ & 1.58 & & & \\
\hline $01: 19 P M$ & $\mathrm{G}^{\prime}$ & 1.59 & & & \\
\hline $01: 31 \mathrm{PM}$ & $G^{\prime}$ & 1.60 & 133 & & \\
\hline $01: 48 \mathrm{PM}$ & $G^{\prime}$ & 1.58 & & & \\
\hline 02:00 PM & $G^{\prime}$ & 1.58 & 120 & & \\
\hline $02: 36 \mathrm{PM}$ & $G^{\prime}$ & 1.62 & & & \\
\hline $02: 53 \mathrm{PM}$ & $G^{\prime}$ & 1.60 & & & \\
\hline $03: 10 P M$ & $\mathrm{G}^{\prime}$ & 1.58 & & & \\
\hline $03: 29 \mathrm{PM}$ & $G^{\prime}$ & 1.59 & 144 & & \\
\hline $03: 47$ PM & $G^{\prime}$ & 1.59 & & & \\
\hline $04: 10 \mathrm{PM}$ & $G^{\prime}$ & 1.59 & & & \\
\hline $04: 22 \mathrm{PM}$ & $G^{\prime}$ & 1.59 & 155 & & \\
\hline 04:38 PM & $G^{\prime}$ & 1.58 & & & \\
\hline & & & & & \\
\hline & & & & & \\
\hline
\end{tabular}




\section{ACT REMBCO JOINT VENTURE \\ Ornl Wag 4 Seeps 4 \& 6 Removal Action Project \\ Quality Contol Report \\ September 23, 1996}

\begin{tabular}{|c|c|c|c|c|c|}
\hline Time of Test & $\begin{array}{c}\text { Mix } \\
\text { Identificaton }\end{array}$ & $\begin{array}{c}\text { Specific } \\
\text { Gravity } \\
\text { (Kg/Litre) }\end{array}$ & $\begin{array}{c}\text { Marsh } \\
\text { Funnel } \\
\text { (seconds) }\end{array}$ & $\begin{array}{l}\text { Coefficient } \\
\text { Pressure } \\
\text { Filtration } \\
\text { (seconds- } 1 / 2 \text { ) }\end{array}$ & $\begin{array}{c}\text { Static } \\
\text { Cone } \\
\text { Penetrometer } \\
(\mathrm{mm})\end{array}$ \\
\hline $08: 45 \mathrm{AM}$ & $M^{\prime \prime}$ & 1.28 & 43 & & \\
\hline $08: 56$ AM & $\mathrm{M}^{\prime \prime}$ & 1.27 & & & \\
\hline $09: 10 \mathrm{AM}$ & $M^{\prime \prime}$ & 1.31 & 44 & & \\
\hline $09: 24$ AM & M" & 1.30 & & & \\
\hline $09: 58$ AM & $M^{\prime \prime}$ & 1.30 & & & \\
\hline $10: 18 \mathrm{AM}$ & $M^{\prime \prime}$ & 1.29 & & & \\
\hline 10:36 AM & $M^{\prime \prime}$ & 1.28 & $\overline{43}$ & & \\
\hline $11: 12$ AM & M" & 1.30 & & & \\
\hline $11: 40 \mathrm{AM}$ & $\mathrm{M}^{\prime \prime}$ & 1.31 & 48 & & \\
\hline 01:00 PM & $M^{\prime \prime}$ & 1.30 & & & \\
\hline $01: 23 \mathrm{PM}$ & $M^{\prime \prime}$ & 1.30 & & & \\
\hline 01:42 PM & M" & 1.30 & 43 & & \\
\hline $02: 05 \mathrm{PM}$ & M" & 1.32 & & & \\
\hline $02: 18 \mathrm{PM}$ & M" & 1.31 & & & \\
\hline $02: 43 \mathrm{PM}$ & M" & 1.25 & & & \\
\hline 03:07 PM & M" & 1.30 & & & \\
\hline $03: 30 \mathrm{PM}$ & M" & 1.30 & 43 & & \\
\hline & & & & & \\
\hline & & & & & \\
\hline
\end{tabular}

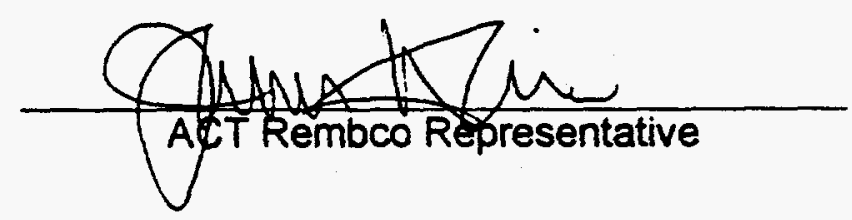




\section{ACT REMBCO JOINT VENTURE \\ Ornl Wag 4 Seeps 4 \& 6 Removal Action Project}

Quality Contol Report

September 24, 1996

\begin{tabular}{|c|c|c|c|c|c|}
\hline Time of Test & $\begin{array}{c}\text { Mix } \\
\text { Identificaton }\end{array}$ & $\begin{array}{c}\text { Specific } \\
\text { Gravity } \\
\text { (Kg/Litre) }\end{array}$ & $\begin{array}{c}\text { Marsh } \\
\text { Funnel } \\
\text { (seconds) }\end{array}$ & $\begin{array}{c}\text { Coefficient } \\
\text { Pressure } \\
\text { Filtration } \\
\text { (seconds-1/2) }\end{array}$ & $\begin{array}{c}\text { Static } \\
\text { Cone } \\
\text { Penetrometer } \\
\text { (mm) }\end{array}$ \\
\hline $08: 22 \mathrm{AM}$ & $M^{\prime \prime}$ & 1.29 & 43 & & \\
\hline $08: 34 \mathrm{AM}$ & $M^{\prime \prime}$ & 1.31 & & & \\
\hline $08: 47 \mathrm{AM}$ & $M^{\prime \prime}$ & 1.31 & 43 & & \\
\hline $09: 10 \mathrm{AM}$ & $M^{\prime \prime}$ & 1.30 & & & \\
\hline $09: 32 \mathrm{AM}$ & $M^{\prime \prime}$ & 1.30 & & & \\
\hline $10: 12 \mathrm{AM}$ & $M^{\prime \prime}$ & 1.30 & & & \\
\hline $10: 26 \mathrm{AM}$ & $M^{\prime \prime}$ & 1.30 & 45 & & \\
\hline $11: 05 \mathrm{AM}$ & $M^{\prime \prime}$ & 1.30 & & & \\
\hline $11: 50 \mathrm{AM}$ & $M^{\prime \prime}$ & 1.30 & 46 & $5.000 \mathrm{E}-02$ & \\
\hline $12: 50 \mathrm{PM}$ & $M^{\prime \prime}$ & 1.30 & & & \\
\hline $01: 22 \mathrm{PM}$ & $M^{\prime \prime}$ & 1.30 & & & \\
\hline $01: 40 \mathrm{PM}$ & $M^{\prime \prime}$ & 1.30 & 45 & & \\
\hline $02: 28 \mathrm{PM}$ & $M^{\prime \prime}$ & 1.28 & & & \\
\hline $02: 52 \mathrm{PM}$ & $M$ & 1.29 & 48 & & \\
\hline $03: 16 \mathrm{PM}$ & $M$ & 1.26 & & & \\
\hline $03: 30 \mathrm{PM}$ & $M$ & 1.27 & & & \\
\hline $03: 58 \mathrm{PM}$ & $M$ & 1.26 & 49 & & \\
\hline $04: 17 \mathrm{PM}$ & $\mathrm{M}$ & 1.54 & 112 & & \\
\hline & & & & & \\
\hline
\end{tabular}

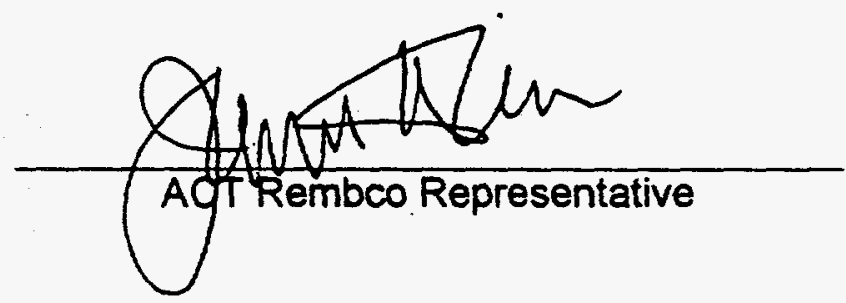




\section{ACT REMBCO JOINT VENTURE \\ Ornl Wag 4 Seeps 4 \& 6 Removal Action Project}

Quality Contol Report

September 25, 1996

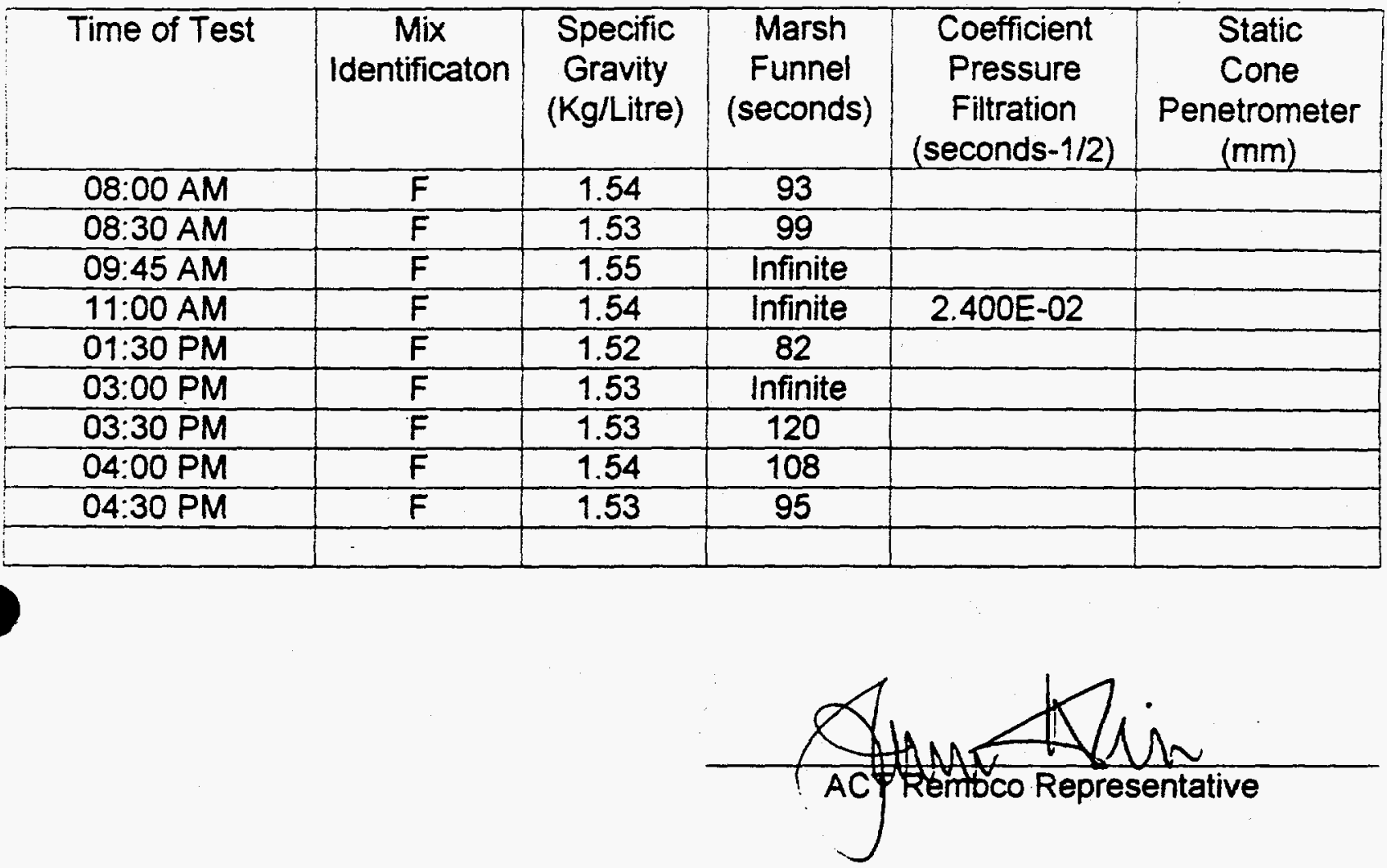




\section{ACT REMBCO JOINT VENTURE \\ Ornl Wag 4 Seeps 4 \& 6 Removal Action Project}

Quality Contol Report

September 26, 1996

\begin{tabular}{|c|c|c|c|c|c|}
\hline Time of Test & $\begin{array}{c}\text { Mix } \\
\text { Identificaton }\end{array}$ & $\begin{array}{c}\text { Specific } \\
\text { Gravity } \\
\text { (Kg/Litre) }\end{array}$ & $\begin{array}{c}\text { Marsh } \\
\text { Funnel } \\
\text { (seconds) }\end{array}$ & $\begin{array}{c}\text { Coefficient } \\
\text { Pressure } \\
\text { Filtration } \\
\text { (seconds-1/2) }\end{array}$ & $\begin{array}{c}\text { Static } \\
\text { Cone } \\
\text { Penetrometer } \\
\text { (mm) }\end{array}$ \\
\hline $08: 30 \mathrm{AM}$ & $\mathrm{G}^{\prime}$ & 1.59 & Infinite & $2.400 \mathrm{E}-02$ & \\
\hline $09: 00 \mathrm{AM}$ & $\mathrm{F}$ & 1.55 & 85 & & \\
\hline $10: 00 \mathrm{AM}$ & $\mathrm{F}$ & 1.53 & 100 & & \\
\hline $11: 03 \mathrm{AM}$ & $\mathrm{F}$ & 1.56 & Infinite & & \\
\hline $11: 11 \mathrm{AM}$ & $\mathrm{F}$ & 1.54 & 120 & & \\
\hline $11: 33 \mathrm{AM}$ & $\mathrm{F}$ & 1.52 & Infinite & & \\
\hline $11: 45 \mathrm{AM}$ & $\mathrm{F}$ & 1.57 & Infinite & & \\
\hline $12: 46 \mathrm{PM}$ & $\mathrm{F}$ & 1.53 & Infinite & & \\
\hline $01: 05 \mathrm{PM}$ & $\mathrm{F}$ & 1.53 & Infinite & & \\
\hline $01: 25 \mathrm{PM}$ & $\mathrm{F}$ & 1.51 & Infinite & & \\
\hline $01: 52 \mathrm{PM}$ & $\mathrm{F}$ & 1.53 & 118 & & \\
\hline $02: 15 \mathrm{PM}$ & $\mathrm{F}$ & 1.57 & Infinite & & \\
\hline $02: 46 \mathrm{PM}$ & $\mathrm{F}$ & 1.51 & 130 & & \\
\hline $03: 07 \mathrm{PM}$ & $\mathrm{F}$ & 1.53 & Infinite & & \\
\hline $03: 20 \mathrm{PM}$ & $\mathrm{F}$ & 1.54 & 113 & & \\
\hline & & & & & \\
\hline & & & & & \\
\hline & & & & & \\
\hline
\end{tabular}

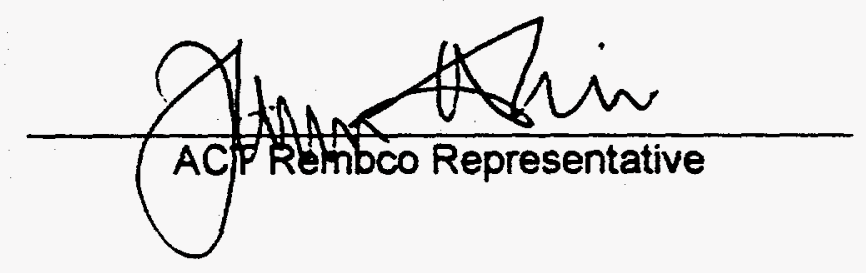




\section{ACT REMBCO JOINT VENTURE \\ Ornl Wag 4 Seeps 4 \& 6 Removal Action Project}

Quality Contol Report

September 27, 1996

\begin{tabular}{|c|c|c|c|c|c|}
\hline Time of Test & $\begin{array}{c}\text { Mix } \\
\text { Identificaton }\end{array}$ & $\begin{array}{c}\text { Specific } \\
\text { Gravity } \\
\text { (Kg/Litre) }\end{array}$ & $\begin{array}{c}\text { Marsh } \\
\text { Funnel } \\
\text { (seconds) }\end{array}$ & $\begin{array}{c}\text { Coefficient } \\
\text { Pressure } \\
\text { Filtration } \\
\text { (seconds-1/2) }\end{array}$ & $\begin{array}{c}\text { Static } \\
\text { Cone } \\
\text { Penetrometer } \\
\text { (mm) }\end{array}$ \\
\hline $09: 05 \mathrm{AM}$ & $\mathrm{M}^{\prime \prime}$ & 1.22 & 43 & & \\
\hline $09: 20 \mathrm{AM}$ & $M^{\prime \prime}$ & 1.24 & 38 & & \\
\hline $10: 08 \mathrm{AM}$ & $\mathrm{M}^{\prime \prime}$ & 1.20 & & & \\
\hline $10: 22 \mathrm{AM}$ & $\mathrm{M}^{\prime \prime}$ & 1.23 & 42 & & \\
\hline $10: 35 \mathrm{AM}$ & $\mathrm{M}^{\prime \prime}$ & 1.23 & & & \\
\hline $10: 42 \mathrm{AM}$ & $\mathrm{M}^{\prime \prime}$ & 1.24 & & & \\
\hline $11: 00 \mathrm{AM}$ & $\mathrm{M}^{\prime \prime}$ & 1.23 & 44 & & \\
\hline $11: 15 \mathrm{AM}$ & $\mathrm{M}^{\prime \prime}$ & 1.24 & & & \\
\hline $01: 13 \mathrm{PM}$ & $\mathrm{F}$ & 1.54 & 110 & & \\
\hline $01: 26 \mathrm{PM}$ & $\mathrm{F}$ & 1.53 & Infinite & & \\
\hline $01: 40 \mathrm{PM}$ & $\mathrm{F}$ & 1.56 & Infinite & & \\
\hline $02: 07 \mathrm{PM}$ & $\mathrm{F}$ & 1.55 & Infinite & & \\
\hline $02: 30 \mathrm{PM}$ & $\mathrm{F}$ & 1.56 & Infinite & & \\
\hline $02: 58 \mathrm{PM}$ & $\mathrm{F}$ & 1.53 & Infinite & & \\
\hline & & & & & \\
\hline & & & & & \\
\hline & & & & & \\
\hline
\end{tabular}

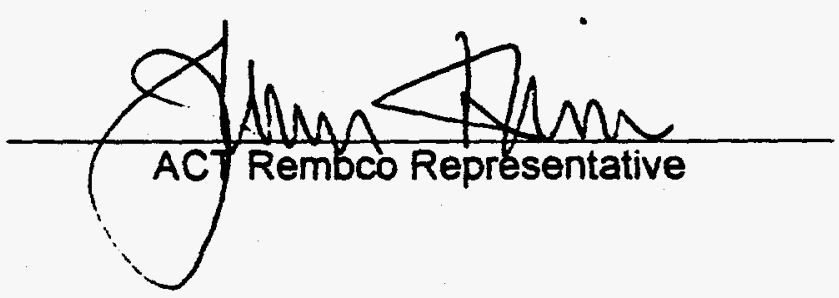


ACT REMBCO JOINT VENTURE

Ornl Wag 4 Seeps 4 \& 6 Removal Action Project

Quality Contol Report

September 30, 1996

\begin{tabular}{|c|c|c|c|c|c|}
\hline Time of Test & $\begin{array}{c}\text { Mix } \\
\text { Identificaton }\end{array}$ & $\begin{array}{c}\text { Specific } \\
\text { Gravity } \\
\text { (Kg/Litre) }\end{array}$ & $\begin{array}{c}\text { Marsh } \\
\text { Funnel } \\
\text { (seconds) }\end{array}$ & $\begin{array}{c}\text { Coefficient } \\
\text { Pressure } \\
\text { Filtration } \\
\text { (seconds-1/2) }\end{array}$ & $\begin{array}{c}\text { Static } \\
\text { Cone } \\
\text { Penetrometer } \\
\text { (mm) }\end{array}$ \\
\hline $08: 30 \mathrm{AM}$ & $M^{\prime \prime}$ & 1.25 & 44 & & \\
\hline $08: 45 \mathrm{AM}$ & $M^{\prime \prime}$ & 1.26 & 43 & & \\
\hline $08: 56 \mathrm{AM}$ & $\mathrm{M}^{\prime \prime}$ & 1.23 & 44 & & \\
\hline $09: 16 \mathrm{AM}$ & $\mathrm{M}^{\prime \prime}$ & 1.23 & 41 & & \\
\hline $09: 35 \mathrm{AM}$ & $M^{\prime \prime}$ & 1.23 & 45 & & \\
\hline $09: 50 \mathrm{AM}$ & $M^{\prime \prime}$ & 1.24 & 47 & & \\
\hline $02: 00 \mathrm{PM}$ & $\mathrm{E}$ & 1.44 & 48 & & \\
\hline $02: 40 \mathrm{PM}$ & $\mathrm{E}$ & 1.45 & & & \\
\hline $03: 00 \mathrm{PM}$ & $\mathrm{E}$ & 1.44 & 51 & & \\
\hline $03: 30 \mathrm{PM}$ & $\mathrm{E}$ & 1.44 & & & \\
\hline & & & & & \\
\hline & & & & & \\
\hline & & & & & \\
\hline & & & & & \\
\hline & & & & & \\
\hline
\end{tabular}

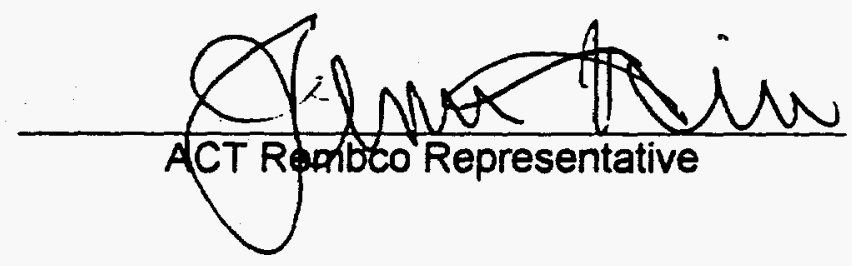




\section{ACT REMBCO JOINT VENTURE Ornl Wag 4 Seeps 4 \& 6 Removal Action Project}

Quality Contol Report

October 01, 1996

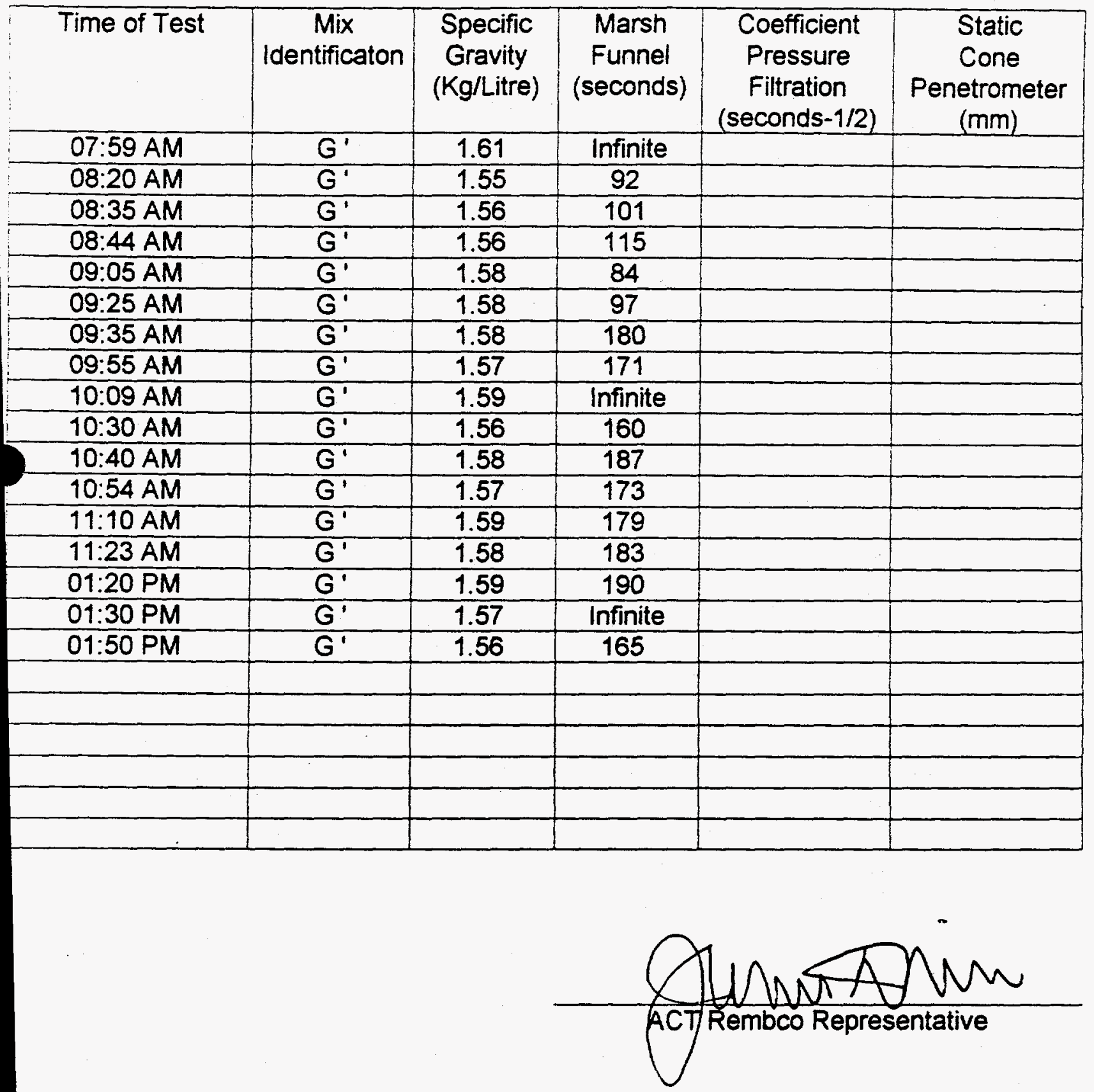




\section{ACT REMBCO JOINT VENTURE \\ Ornl Wag 4 Seeps 4 \& 6 Removal Action Project \\ Quality Contol Report \\ October 03,1996}

\begin{tabular}{|c|c|c|c|c|c|}
\hline Time of Test & $\begin{array}{c}\text { Mix } \\
\text { Identificaton }\end{array}$ & $\begin{array}{c}\text { Specific } \\
\text { Gravity } \\
\text { (Kg/Litre) }\end{array}$ & $\begin{array}{c}\text { Marsh } \\
\text { Funnel } \\
\text { (seconds) }\end{array}$ & $\begin{array}{c}\text { Coefficient } \\
\text { Pressure } \\
\text { Filtration } \\
\text { (seconds-1/2) }\end{array}$ & $\begin{array}{c}\text { Static } \\
\text { Cone } \\
\text { Penetrometer } \\
\text { (mm) }\end{array}$ \\
\hline $01: 00 \mathrm{PM}$ & $\mathrm{G}^{\prime}$ & 1.60 & 133 & & \\
\hline $01: 14 \mathrm{PM}$ & $\mathrm{G}^{\prime}$ & 1.61 & Infinite & & \\
\hline $01: 27 \mathrm{PM}$ & $\mathrm{G}^{\prime}$ & 1.59 & 102 & & \\
\hline $01: 41 \mathrm{PM}$ & $\mathrm{G}^{\prime}$ & 1.58 & & & \\
\hline $01: 55 \mathrm{PM}$ & $\mathrm{G}^{\prime}$ & 1.59 & & & \\
\hline $02: 15 \mathrm{PM}$ & $\mathrm{G}^{\prime}$ & 1.59 & 100 & & \\
\hline $02: 36 \mathrm{PM}$ & $\mathrm{G}^{\prime}$ & 1.59 & & & \\
\hline $02: 55 \mathrm{PM}$ & $\mathrm{G}^{\prime}$ & 1.59 & & & \\
\hline $03: 10 \mathrm{PM}$ & $\mathrm{G}^{\prime}$ & 1.55 & Infinite & & \\
\hline $03: 22 \mathrm{PM}$ & $\mathrm{G}^{\prime}$ & 1.60 & Infinite & & \\
\hline $03: 30 \mathrm{PM}$ & $\mathrm{G}^{\prime}$ & 1.60 & Infinite & & \\
\hline $03: 37 \mathrm{PM}$ & $\mathrm{G}^{\prime}$ & 1.58 & & & \\
\hline $04: 10 \mathrm{PM}$ & $\mathrm{G}^{\prime}$ & 1.59 & 156 & & \\
\hline & & & & & \\
\hline & & & & & \\
\hline & & & & & \\
\hline
\end{tabular}

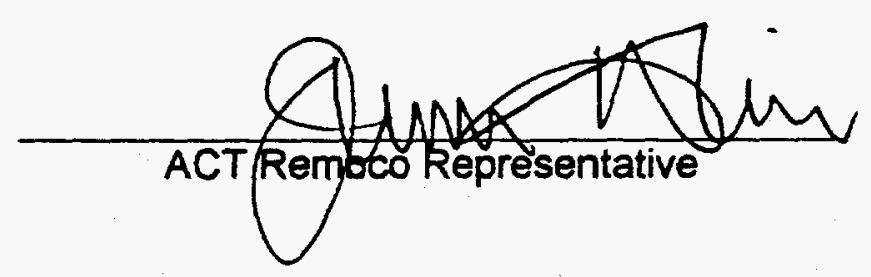




\section{ACT REMBCO JOINT VENTURE \\ Ornl Wag 4 Seeps 4 \& 6 Removal Action Project \\ Quality Contol Report \\ October 04, 1996}

\begin{tabular}{|c|c|c|c|c|c|}
\hline Time of Test & $\begin{array}{c}\text { Mix } \\
\text { Identificaton }\end{array}$ & $\begin{array}{c}\text { Specific } \\
\text { Gravity } \\
\text { (Kg/Litre) }\end{array}$ & $\begin{array}{c}\text { Marsh } \\
\text { Funnel } \\
\text { (seconds) }\end{array}$ & $\begin{array}{l}\text { Coefficient } \\
\text { Pressure } \\
\text { Filtration } \\
\text { (seconds-1/2) }\end{array}$ & $\begin{array}{c}\text { Static } \\
\text { Cone } \\
\text { Penetrometer } \\
(\mathrm{mm})\end{array}$ \\
\hline $08: 06$ AM & $G^{\prime}$ & 1.59 & Infinite & & \\
\hline $08: 18 \mathrm{AM}$ & $\mathrm{G}^{\prime}$ & 1.54 & Infinite & & \\
\hline $08: 35 \mathrm{AM}$ & $G^{\prime}$ & 1.58 & Infinite & & \\
\hline $08: 58 \mathrm{AM}$ & $\mathrm{G}^{\prime}$ & 1.60 & 180 & & \\
\hline $09: 40 \mathrm{AM}$ & $F$ & 1.51 & 125 & & \\
\hline $09: 10 \mathrm{AM}$ & M" & 1.20 & 45 & & \\
\hline $09: 55 \mathrm{AM}$ & M" & 1.21 & 46 & & \\
\hline 10:00 AM & $F$ & 1.52 & 95 & & \\
\hline $10: 24 \mathrm{AM}$ & $F$ & 1.52 & 99 & & \\
\hline $10: 38 \mathrm{AM}$ & $F$ & 1.53 & 100 & & \\
\hline $10: 49 \mathrm{AM}$ & M" & 1.22 & 48 & & \\
\hline $10: 56 \mathrm{AM}$ & $F$ & 1.51 & 88 & & \\
\hline 11:06 AM & $F$ & 1.53 & 83 & & \\
\hline $11: 17 \mathrm{AM}$ & $F$ & 1.52 & 92 & & \\
\hline 11:37 AM & $F$ & 1.53 & 96 & & \\
\hline $12: 51 \mathrm{PM}$ & $F$ & 1.54 & 101 & & \\
\hline 01:05 PM & $F$ & 1.54 & 99 & & \\
\hline 01:20 PM & $F$ & 1.53 & 90 & & \\
\hline $01: 30 \mathrm{PM}$ & $F$ & 1.55 & 95 & & \\
\hline $01: 45 \mathrm{PM}$ & $F$ & 1.53 & 88 & & \\
\hline $02: 00 \mathrm{PM}$ & $F$ & 1.53 & 94 & & \\
\hline $02: 15 \mathrm{PM}$ & $F$ & 1.53 & 97 & & \\
\hline 02:30 PM & $F$ & 1.53 & 110 & & \\
\hline $02: 45 \mathrm{PM}$ & $F$ & 1.52 & 120 & & \\
\hline 03:01 PM & $F$ & 1.54 & Infinite & & \\
\hline 03:20 PM & $\bar{F}$ & 1.54 & 129 & & \\
\hline 03:35 PM & $F$ & 1.54 & 140 & & \\
\hline 03:53 PM & $F$ & 1.52 & 162 & & \\
\hline
\end{tabular}




\section{ACT REMBCO JOINT VENTURE Ornl Wag 4 Seeps 4 \& 6 Removal Action Project}

Quality Contol Report

October 07.1996

\begin{tabular}{|c|c|c|c|c|c|}
\hline Time of Test & $\begin{array}{c}\text { Mix } \\
\text { Identificaton }\end{array}$ & $\begin{array}{c}\text { Specific } \\
\text { Gravity } \\
\text { (Kg/Litre) }\end{array}$ & $\begin{array}{c}\text { Marsh } \\
\text { Funnel } \\
\text { (seconds) }\end{array}$ & $\begin{array}{c}\text { Coefficient } \\
\text { Pressure } \\
\text { Filtration } \\
\text { (seconds-1/2) }\end{array}$ & $\begin{array}{c}\text { Static } \\
\text { Cone } \\
\text { Penetrometer } \\
\text { (mm) }\end{array}$ \\
\hline $08: 10 \mathrm{AM}$ & $\mathrm{F}$ & 1.53 & 90 & & \\
\hline $08: 21 \mathrm{AM}$ & $\mathrm{F}$ & 1.51 & 93 & & \\
\hline $08: 41 \mathrm{AM}$ & $\mathrm{F}$ & 1.52 & 89 & & \\
\hline $09: 00 \mathrm{AM}$ & $\mathrm{F}$ & 1.53 & 96 & & \\
\hline $09: 12 \mathrm{AM}$ & $\mathrm{F}$ & 1.52 & 80 & & \\
\hline $09: 25 \mathrm{AM}$ & $\mathrm{F}$ & 1.51 & 88 & & \\
\hline $09: 36 \mathrm{AM}$ & $\mathrm{F}$ & 1.52 & 108 & & \\
\hline $09: 50 \mathrm{AM}$ & $\mathrm{F}$ & 1.53 & & & \\
\hline $10: 02 \mathrm{AM}$ & $\mathrm{F}$ & 1.53 & 97 & & \\
\hline $10: 13 \mathrm{AM}$ & $\mathrm{F}$ & 1.53 & & & \\
\hline $10: 22 \mathrm{AM}$ & $\mathrm{F}$ & 1.53 & 99 & & \\
\hline $10: 33 \mathrm{AM}$ & $\mathrm{F}$ & 1.53 & & & \\
\hline $10: 50 \mathrm{AM}$ & $\mathrm{F}$ & 1.53 & Infinite & & \\
\hline $11: 04 \mathrm{AM}$ & $\mathrm{F}$ & 1.54 & & & \\
\hline $11: 19 \mathrm{AM}$ & $\mathrm{F}$ & 1.54 & Infinite & & \\
\hline $11: 33 \mathrm{AM}$ & $\mathrm{F}$ & 1.53 & & & \\
\hline $11: 46 \mathrm{AM}$ & $\mathrm{F}$ & 1.53 & 120 & & \\
\hline $12: 40 \mathrm{PM}$ & $\mathrm{F}$ & 1.53 & & & \\
\hline $12: 55 \mathrm{PM}$ & $\mathrm{F}$ & 1.53 & 107 & & \\
\hline $01: 05 \mathrm{PM}$ & $\mathrm{F}$ & 1.52 & 140 & & \\
\hline $01: 23 \mathrm{PM}$ & $\mathrm{F}$ & 1.53 & & & \\
\hline $01: 35 \mathrm{PM}$ & $\mathrm{F}$ & 1.53 & 121 & & \\
\hline $01: 49 \mathrm{PM}$ & $\mathrm{F}$ & 1.53 & & & \\
\hline $02: 10 \mathrm{PM}$ & $\mathrm{F}$ & 1.51 & 115 & & \\
\hline $02: 20 \mathrm{PM}$ & $\mathrm{F}$ & 1.52 & & & \\
\hline $02: 37 \mathrm{PM}$ & $\mathrm{F}$ & 1.54 & 56 & & \\
\hline $02: 47 \mathrm{PM}$ & $\mathrm{F}$ & 1.53 & & & \\
\hline & & & & & \\
\hline & & & & \\
\hline & & & & \\
\hline & & & & \\
\hline & & & & \\
\hline & & & & \\
\hline & & & & \\
\hline & & & & \\
\hline
\end{tabular}




\section{ACT REMBCO JOINT VENTURE \\ Ornl Wag 4 Seeps 4 \& 6 Removal Action Project \\ Quality Contol Report \\ October 07, 1996}

\begin{tabular}{|c|c|c|c|c|c|}
\hline Time of Test & $\begin{array}{c}\text { Mix } \\
\text { Identificaton }\end{array}$ & $\begin{array}{c}\text { Specific } \\
\text { Gravity } \\
\text { (Kg/Litre) }\end{array}$ & $\begin{array}{l}\text { Marsh } \\
\text { Funnel } \\
\text { (seconds) }\end{array}$ & $\begin{array}{l}\text { Coefficient } \\
\text { Pressure } \\
\text { Filtration } \\
\text { (seconds-1/2) }\end{array}$ & $\begin{array}{c}\text { Static } \\
\text { Cone } \\
\text { Penetrometer } \\
(\mathrm{mm})\end{array}$ \\
\hline 03:03 PM & $F$ & 1.52 & 76 & & \\
\hline $03: 16 \mathrm{PM}$ & $\bar{F}$ & 1.53 & & & \\
\hline $03: 35 \mathrm{PM}$ & $\bar{F}$ & $1.5 \overline{3}$ & 83 & & \\
\hline $03: 45 \mathrm{PM}$ & $F$ & 1.53 & & & \\
\hline $04: 00 \mathrm{PM}$ & $\bar{F}$ & 1.53 & 79 & & \\
\hline $04: 20 \mathrm{PM}$ & $F$ & 1.52 & & & \\
\hline $03: 35 \mathrm{PM}$ & $F$ & 1.51 & 92 & & \\
\hline & & & & & \\
\hline & & & & & \\
\hline & & & & & \\
\hline & & & & & \\
\hline & & & & & ntative \\
\hline
\end{tabular}




\section{ACT REMBCO JOINT VENTURE \\ Ornl Wag 4 Seeps 4 \& 6 Removal Action Project \\ Quality Contol Report \\ October 08. 1996}

\begin{tabular}{|c|c|c|c|c|c|}
\hline Time of Test & $\begin{array}{c}\text { Mix } \\
\text { Identificaton }\end{array}$ & $\begin{array}{c}\text { Specific } \\
\text { Gravity } \\
\text { (Kg/Litre) }\end{array}$ & $\begin{array}{c}\text { Marsh } \\
\text { Funnel } \\
\text { (seconds) }\end{array}$ & $\begin{array}{l}\text { Coefficient } \\
\text { Pressure } \\
\text { Filtration } \\
\text { (minutes-1/2) }\end{array}$ & $\begin{array}{c}\text { Static } \\
\text { Cone } \\
\text { Penetrometer } \\
(\mathrm{mm})\end{array}$ \\
\hline $08: 20 \mathrm{AM}$ & $M^{\prime \prime}$ & 1.19 & 40 & & \\
\hline $08: 30 \mathrm{AM}$ & $M^{\prime \prime}$ & 1.24 & & & \\
\hline $08: 40 \mathrm{AM}$ & $M^{\prime \prime}$ & 1.22 & 45 & & \\
\hline $08: 52 \mathrm{AM}$ & $M^{\prime \prime}$ & 1.23 & & & \\
\hline $09: 08 \mathrm{AM}$ & $M^{\prime \prime}$ & 1.20 & 42 & . & \\
\hline 09:20 AM & $M^{\prime \prime}$ & 1.22 & & & \\
\hline $09: 38 \mathrm{AM}$ & $M^{\prime \prime}$ & 1.25 & 43 & & \\
\hline $09: 50 \mathrm{AM}$ & M" & 1.24 & & & \\
\hline 10:00 AM & $M^{\prime \prime}$ & 1.23 & 44 & & \\
\hline $10: 10 \mathrm{AM}$ & $M^{\prime \prime}$ & 1.23 & & & \\
\hline $10: 27 \mathrm{AM}$ & M" & 1.23 & 45 & & \\
\hline $10: 48 \mathrm{AM}$ & M" & 1.23 & & & \\
\hline $10: 59 \mathrm{AM}$ & M" & 1.23 & 44 & & \\
\hline $11: 14 \mathrm{AM}$ & $M^{\prime \prime}$ & 1.21 & & & \\
\hline $11: 26$ AM & $\mathrm{M}^{\prime \prime}$ & 1.22 & 42 & & \\
\hline $11: 36 \mathrm{AM}$ & M" & 1.22 & & & \\
\hline $11: 47 \mathrm{AM}$ & $M^{\prime \prime}$ & 1.22 & 44 & & \\
\hline $12: 57 \mathrm{PM}$ & M" & 1.22 & & & \\
\hline $01: 08 \mathrm{PM}$ & $M^{\prime \prime}$ & 1.22 & 44 & & \\
\hline $01: 20 \mathrm{PM}$ & $M^{\prime \prime}$ & 1.23 & & & \\
\hline $01: 30 \mathrm{PM}$ & $\mathrm{M}^{\prime \prime}$ & 1.23 & 45 & & \\
\hline $01: 57 \mathrm{PM}$ & $M^{\prime \prime}$ & 1.23 & & & \\
\hline $02: 07 \mathrm{PM}$ & $M^{\prime \prime}$ & 1.26 & $\overline{44}$ & $4.500 \mathrm{E}-02$ & \\
\hline $02: 37 \mathrm{PM}$ & $M^{\prime \prime}$ & 1.22 & & & \\
\hline $02: 51 \mathrm{PM}$ & $\mathrm{M}^{\prime \prime}$ & 1.23 & 44 & & \\
\hline 03:10 PM & $M^{\prime \prime}$ & 1.23 & & & \\
\hline $03: 20 \mathrm{PM}$ & $M^{\prime \prime}$ & 1.22 & 42 & & \\
\hline
\end{tabular}




\section{ACT REMBCO JOINT VENTURE \\ Ornl Wag 4 Seeps 4 \& 6 Removal Action Project}

Quality Contol Report

October 08, 1996

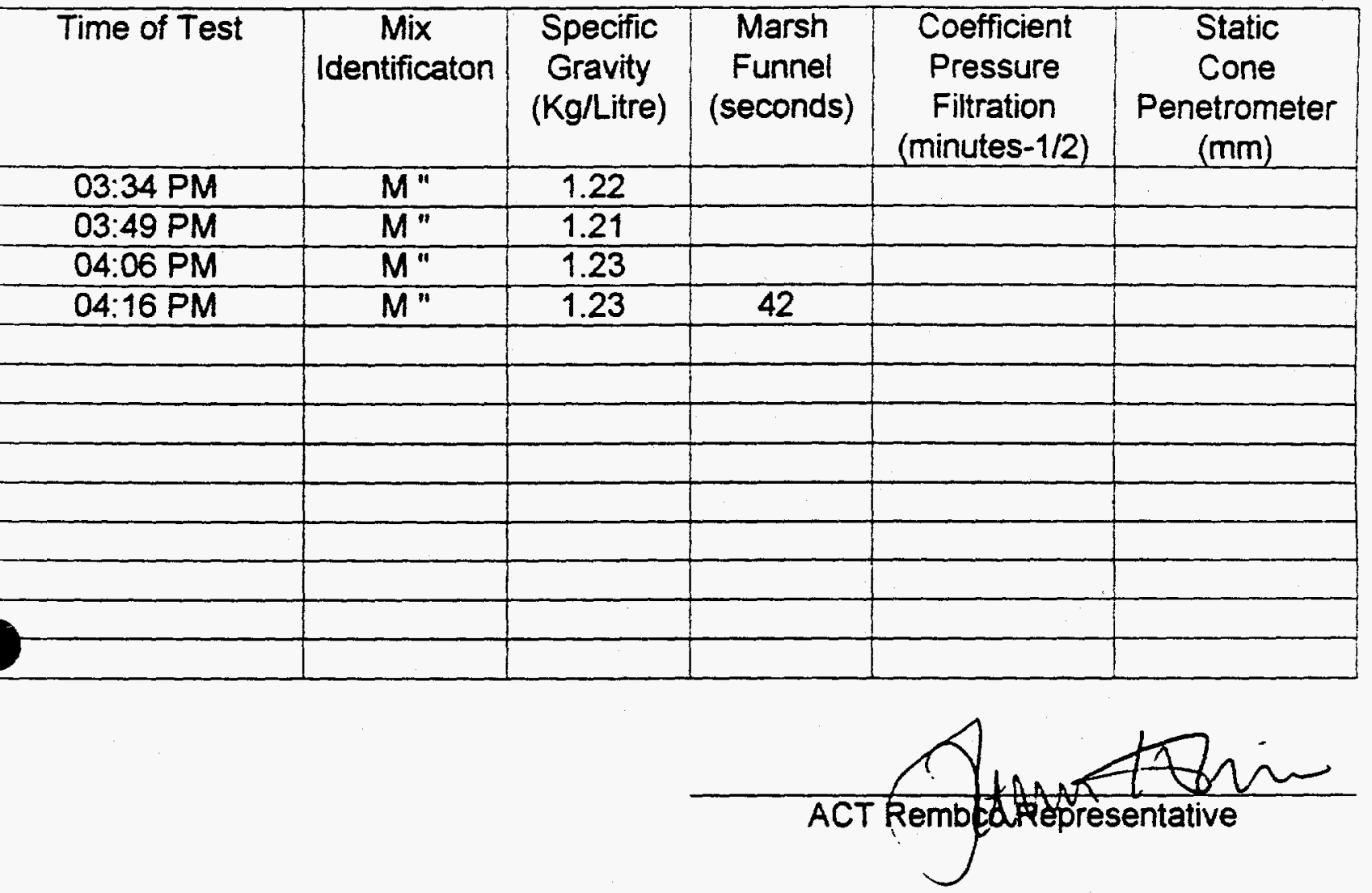




\section{ACT REMBCO JOINT VENTURE \\ Ornl Wag 4 Seeps 4 \& 6 Removal Action Project \\ Quality Contol Report \\ October 09, 1996}

\begin{tabular}{|c|c|c|c|c|c|}
\hline Time of Test & $\begin{array}{c}\text { Mix } \\
\text { Identificaton }\end{array}$ & $\begin{array}{l}\text { Specific } \\
\text { Gravity } \\
\text { (Kg/Litre) }\end{array}$ & $\begin{array}{c}\text { Marsh } \\
\text { Funnel } \\
\text { (seconds) }\end{array}$ & $\begin{array}{c}\text { Coefficient } \\
\text { Pressure } \\
\text { Filtration } \\
\text { (minutes-1/2) }\end{array}$ & $\begin{array}{c}\text { Static } \\
\text { Cone } \\
\text { Penetrometer } \\
(\mathrm{mm})\end{array}$ \\
\hline $08: 10 \mathrm{AM}$ & $F$ & 1.51 & 84 & & \\
\hline $08: 20 \mathrm{AM}$ & $F$ & 1.52 & & & \\
\hline $08: 33 \mathrm{AM}$ & $\bar{F}$ & 1.50 & 87 & & \\
\hline $08: 43 \mathrm{AM}$ & $F$ & 1.53 & & & \\
\hline $08: 55 \mathrm{AM}$ & $F$ & 1.53 & 91 & & \\
\hline $09: 05 \mathrm{AM}$ & $F$ & 1.54 & & & \\
\hline $09: 15 \mathrm{AM}$ & $F$ & 1.54 & 84 & & \\
\hline $09: 25 \mathrm{AM}$ & $F$ & 1.53 & & & \\
\hline $09: 35 \mathrm{AM}$ & $\bar{F}$ & 1.53 & 92 & & \\
\hline $09: 45 \mathrm{AM}$ & $F$ & 1.53 & & & \\
\hline $09: 55 \mathrm{AM}$ & $F$ & 1.53 & 120 & & \\
\hline $10: 10 \mathrm{AM}$ & $F$ & 1.53 & & $2.500 \mathrm{E}-02$ & \\
\hline $10: 20 \mathrm{AM}$ & $F$ & 1.53 & 115 & & \\
\hline 10:30 AM & $F$ & 1.53 & & & \\
\hline $10: 55 \mathrm{AM}$ & $F$ & 1.51 & 119 & & \\
\hline $11: 05 \mathrm{AM}$ & $\bar{F}$ & 1.53 & & & \\
\hline $11: 15 \mathrm{AM}$ & $F$ & 1.54 & 111 & & \\
\hline $11: 30 \mathrm{AM}$ & $F$ & 1.54 & & & \\
\hline $11: 45 \mathrm{AM}$ & $F$ & 1.57 & Infinite & & \\
\hline $12: 40 \mathrm{PM}$ & $F$ & 1.53 & Infinite & & \\
\hline $12: 50 \mathrm{PM}$ & $F$ & 1.53 & & & \\
\hline $01: 01 \mathrm{PM}$ & $F$ & 1.53 & Infinite & & \\
\hline $01: 15 \mathrm{PM}$ & $F$ & 1.52 & & & \\
\hline $01: 25 \mathrm{PM}$ & $F$ & 1.53 & 128 & & \\
\hline $01: 35 \mathrm{PM}$ & $F$ & 1.53 & & & \\
\hline $01: 45 \mathrm{PM}$ & $\bar{F}$ & 1.56 & 121 & & \\
\hline $01: 55 \mathrm{PM}$ & $\bar{F}$ & 1.55 & & & \\
\hline
\end{tabular}




\section{ACT REMBCO JOINT VENTURE \\ Ornl Wag 4 Seeps 4 \& 6 Removal Action Project}

Quality Contol Report

October 09, 1996

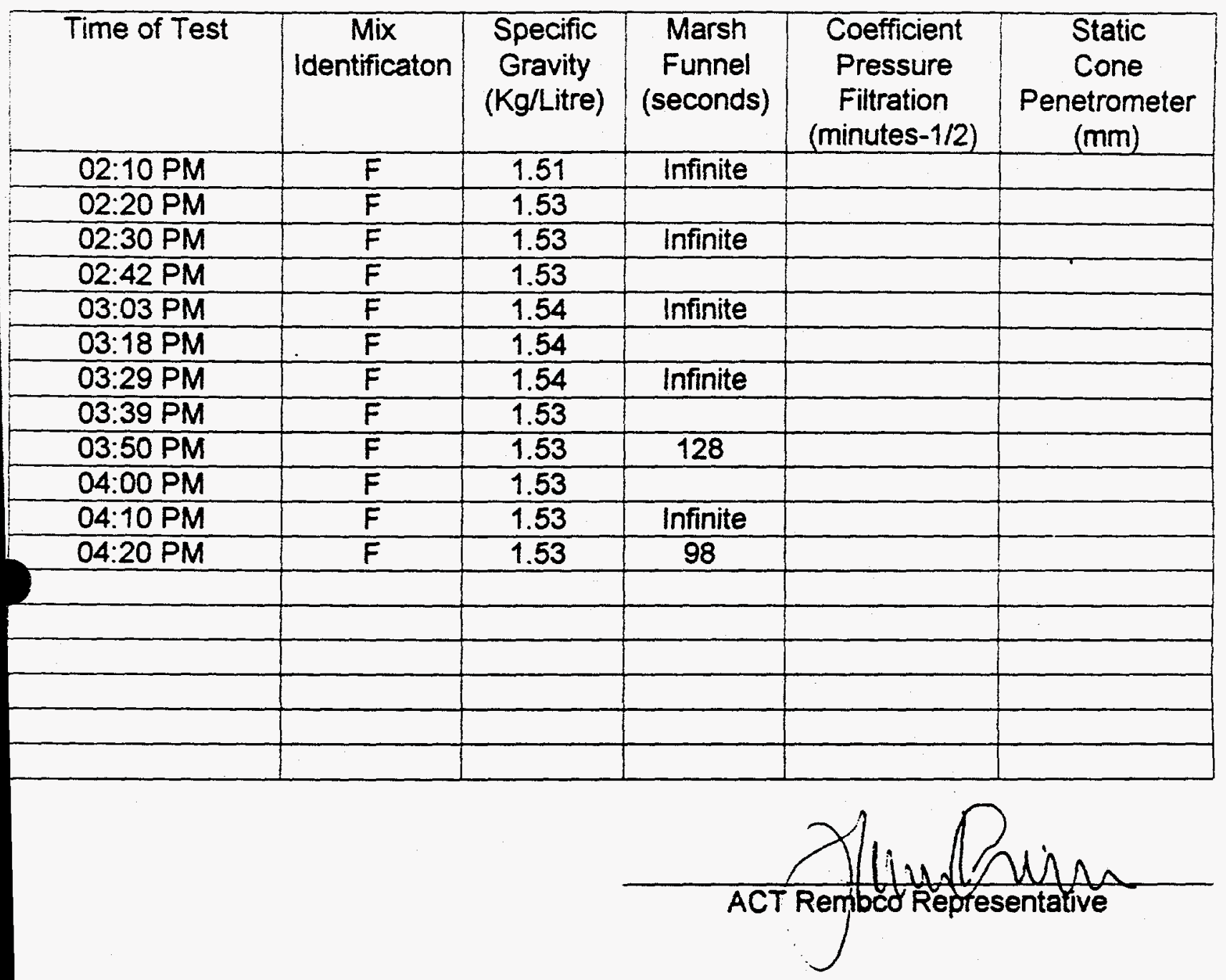




\section{ACT REMBCO JOINT VENTURE \\ Ornl Wag 4 Seeps 4 \& 6 Removal Action Project}

Quality Contol Report

October 10, 1996

\begin{tabular}{|c|c|c|c|c|c|}
\hline Time of Test & $\begin{array}{c}\text { Mix } \\
\text { Identificaton }\end{array}$ & $\begin{array}{c}\text { Specific } \\
\text { Gravity } \\
\text { (Kg/Litre) }\end{array}$ & $\begin{array}{c}\text { Marsh } \\
\text { Funnel } \\
\text { (seconds) }\end{array}$ & $\begin{array}{c}\text { Coefficient } \\
\text { Pressure } \\
\text { Filtration } \\
\text { (minutes-1/2) }\end{array}$ & $\begin{array}{c}\text { Static } \\
\text { Cone } \\
\text { Penetrometer } \\
\text { (mm) }\end{array}$ \\
\hline $08: 00 \mathrm{AM}$ & $\mathrm{F}$ & 1.51 & 91 & & \\
\hline $08: 10 \mathrm{AM}$ & $\mathrm{F}$ & 1.53 & & & \\
\hline $08: 20 \mathrm{AM}$ & $\mathrm{F}$ & 1.54 & 86 & & \\
\hline $08: 30 \mathrm{AM}$ & $\mathrm{F}$ & 1.54 & & & \\
\hline $08: 40 \mathrm{AM}$ & $\mathrm{F}$ & 1.57 & & & \\
\hline $08: 50 \mathrm{AM}$ & $\mathrm{F}$ & 1.53 & & & \\
\hline $09: 00 \mathrm{AM}$ & $\mathrm{F}$ & 1.53 & 95 & & \\
\hline $09: 10 \mathrm{AM}$ & $\mathrm{F}$ & 1.53 & & & \\
\hline $09: 20 \mathrm{AM}$ & $\mathrm{F}$ & 1.52 & & & \\
\hline $09: 30 \mathrm{AM}$ & $\mathrm{F}$ & 1.53 & & & \\
\hline $09: 40 \mathrm{AM}$ & $\mathrm{F}$ & 1.53 & 77 & & \\
\hline $09: 50 \mathrm{AM}$ & $\mathrm{F}$ & 1.56 & & & \\
\hline $10: 00 \mathrm{AM}$ & $\mathrm{F}$ & 1.55 & & & \\
\hline $10: 12 \mathrm{AM}$ & $\mathrm{F}$ & 1.51 & & & \\
\hline $10: 30 \mathrm{AM}$ & $\mathrm{F}$ & 1.53 & 120 & & \\
\hline $10: 40 \mathrm{AM}$ & $\mathrm{F}$ & 1.51 & & & \\
\hline $10: 50 \mathrm{AM}$ & $\mathrm{F}$ & 1.53 & & & \\
\hline $11: 00 \mathrm{AM}$ & $\mathrm{F}$ & 1.53 & & & \\
\hline $11: 10 \mathrm{AM}$ & $\mathrm{F}$ & 1.53 & 100 & & \\
\hline $11: 20 \mathrm{AM}$ & $\mathrm{F}$ & 1.56 & & & \\
\hline $11: 30 \mathrm{AM}$ & $\mathrm{F}$ & 1.55 & & & \\
\hline $11: 42 \mathrm{AM}$ & $\mathrm{F}$ & 1.51 & & & \\
\hline $12: 40 \mathrm{PM}$ & $\mathrm{F}$ & 1.53 & Infinite & & \\
\hline $12: 50 \mathrm{PM}$ & $\mathrm{F}$ & 1.51 & & & \\
\hline $01: 00 \mathrm{PM}$ & $\mathrm{F}$ & 1.53 & & & \\
\hline $01: 10 \mathrm{PM}$ & $\mathrm{F}$ & 1.54 & & & \\
\hline $01: 20 \mathrm{PM}$ & $\mathrm{F}$ & 1.54 & 135 & & \\
\hline $01: 30 \mathrm{PM}$ & $\mathrm{F}$ & 1.53 & & & \\
\hline & & & & & \\
\hline & & & & \\
\hline & & & & \\
\hline & & & & \\
\hline & & & & \\
\hline & & & & \\
\hline & & & & \\
\hline & & & & \\
\hline
\end{tabular}




\section{ACT REMBCO JOINT VENTURE \\ Ornl Wag 4 Seeps 4 \& 6 Removal Action Project}

Quality Contol Report

October 10, 1996

\begin{tabular}{|c|c|c|c|c|c|}
\hline Time of Test & $\begin{array}{c}\text { Mix } \\
\text { Identificaton }\end{array}$ & $\begin{array}{c}\text { Specific } \\
\text { Gravity } \\
\text { (Kg/Litre) }\end{array}$ & $\begin{array}{c}\text { Marsh } \\
\text { Funnel } \\
\text { (seconds) }\end{array}$ & $\begin{array}{c}\text { Coefficient } \\
\text { Pressure } \\
\text { Filtration } \\
\text { (minutes-1/2) }\end{array}$ & $\begin{array}{c}\text { Static } \\
\text { Cone } \\
\text { Penetrometer } \\
\text { (mm) }\end{array}$ \\
\hline $01: 40 \mathrm{PM}$ & $\mathrm{F}$ & 1.53 & & & \\
\hline $01: 50 \mathrm{PM}$ & $\mathrm{F}$ & 1.53 & 110 & $2.600 \mathrm{E}-02$ & \\
\hline $02: 00 \mathrm{PM}$ & $\mathrm{F}$ & 1.53 & & & \\
\hline $02: 10 \mathrm{PM}$ & $\mathrm{F}$ & 1.53 & & & \\
\hline $02: 25 \mathrm{PM}$ & $\mathrm{F}$ & 1.53 & & & \\
\hline $02: 40 \mathrm{PM}$ & $\mathrm{F}$ & 1.54 & Infinite & & \\
\hline $02: 50 \mathrm{PM}$ & $\mathrm{F}$ & 1.54 & & & \\
\hline $03: 00 \mathrm{PM}$ & $\mathrm{F}$ & 1.53 & & & \\
\hline $03: 20 \mathrm{PM}$ & $\mathrm{F}$ & 1.53 & & & \\
\hline $03: 30 \mathrm{PM}$ & $\mathrm{F}$ & 1.53 & Infinite & & \\
\hline $03: 40 \mathrm{PM}$ & $\mathrm{F}$ & 1.53 & & & \\
\hline $03: 50 \mathrm{PM}$ & $\mathrm{F}$ & 1.53 & & & \\
\hline $04: 00 \mathrm{PM}$ & $\mathrm{F}$ & 1.53 & & & \\
\hline $04: 15 \mathrm{PM}$ & $\mathrm{F}$ & 1.53 & 145 & & \\
\hline & & & & & \\
\hline & & & & & \\
\hline & & & & & \\
\hline
\end{tabular}

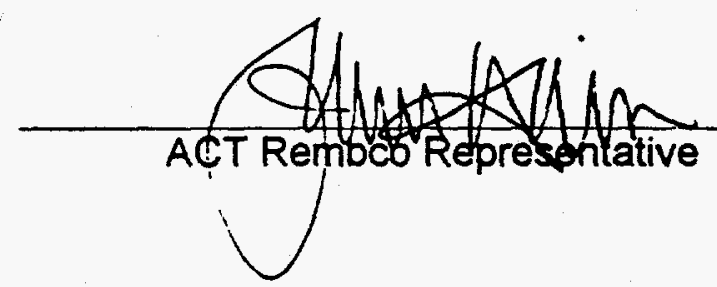


ACT REMBCO JOINT VENTURE

Ornl Wag 4 Seeps 4 \& 6 Removal Action Project

Quality Contol Report

October 11, 1996

\begin{tabular}{|c|c|c|c|c|c|}
\hline Time of Test & $\begin{array}{c}\text { Mix } \\
\text { Identificaton }\end{array}$ & $\begin{array}{c}\text { Specific } \\
\text { Gravity } \\
\text { (Kg/Litre) }\end{array}$ & $\begin{array}{l}\text { Marsh } \\
\text { Funnel } \\
\text { (seconds) }\end{array}$ & $\begin{array}{c}\text { Coefficient } \\
\text { Pressure } \\
\text { Filtration } \\
\text { (minutes-1/2) }\end{array}$ & $\begin{array}{c}\text { Static } \\
\text { Cone } \\
\text { Penetrometer } \\
(\mathrm{mm})\end{array}$ \\
\hline $08: 10 \mathrm{AM}$ & $M^{\prime \prime}$ & 1.23 & 46 & & \\
\hline $08: 20 \mathrm{AM}$ & M" & 1.22 & & & \\
\hline $08: 30$ AM & $M^{\prime \prime}$ & 1.25 & 48 & & \\
\hline $08: 40 \mathrm{AM}$ & $M^{\prime \prime}$ & 1.26 & & & \\
\hline $08: 50 \mathrm{AM}$ & M" & 1.23 & 45 & & \\
\hline $09: 00 \mathrm{AM}$ & $M^{\prime \prime}$ & 1.24 & & & \\
\hline $09: 10 \mathrm{AM}$ & M" & 1.23 & 47 & & \\
\hline $09: 20 \mathrm{AM}$ & M" & 1.22 & & & \\
\hline $09: 30 \mathrm{AM}$ & $M^{\prime \prime}$ & 1.23 & 44 & & \\
\hline $09: 40 \mathrm{AM}$ & $M^{\prime \prime}$ & 1.24 & & & \\
\hline $09: 52$ AM & M" & 1.26 & 52 & \multicolumn{2}{|c|}{ Start Using Microcem B } \\
\hline $10: 02 \mathrm{AM}$ & $M^{\prime \prime}$ & 1.23 & & & \\
\hline $11: 24 \mathrm{AM}$ & $F$ & 1.54 & Infinite & & \\
\hline $11: 34 \mathrm{AM}$ & $F$ & 1.54 & & & \\
\hline $11: 45 \mathrm{AM}$ & $F$ & 1.54 & Infinite & & \\
\hline $11: 55 \mathrm{AM}$ & $\bar{F}$ & 1.53 & & & \\
\hline $12: 45 \mathrm{PM}$ & $\bar{F}$ & 1.55 & Infinite & & \\
\hline $01: 00 \mathrm{PM}$ & $F$ & 1.55 & & & \\
\hline $01: 10 \mathrm{PM}$ & $\bar{F}$ & 1.54 & Infinite & & \\
\hline $01: 20 \mathrm{PM}$ & $\bar{F}$ & 1.54 & & & \\
\hline $01: 30 \mathrm{PM}$ & $F$ & 1.54 & Infinite & & \\
\hline $01: 40 \mathrm{PM}$ & $F$ & 1.54 & & & \\
\hline 02:00 PM & $F$ & 1.54 & Infinite & & \\
\hline $02: 10 \mathrm{PM}$ & $F$ & 1.53 & & & \\
\hline $02: 20 \mathrm{PM}$ & $\bar{F}$ & 1.55 & Infinite & & \\
\hline $02: 30 \mathrm{PM}$ & $F$ & 1.54 & & & \\
\hline $02: 40 \mathrm{PM}$ & $\bar{F}$ & 1.53 & Infinite & & \\
\hline
\end{tabular}




\section{ACT REMBCO JOINT VENTURE \\ Ornl Wag 4 Seeps 4 \& 6 Removal Action Project}

Quality Contol Report

October 11,1996

\begin{tabular}{|c|c|c|c|c|c|}
\hline Time of Test & $\begin{array}{c}\text { Mix } \\
\text { Identificaton }\end{array}$ & $\begin{array}{c}\text { Specific } \\
\text { Gravity } \\
\text { (Kg/Litre) }\end{array}$ & $\begin{array}{l}\text { Marsh } \\
\text { Funnel } \\
\text { (seconds) }\end{array}$ & $\begin{array}{c}\text { Coefficient } \\
\text { Pressure } \\
\text { Filtration } \\
\text { (minutes-1/2) }\end{array}$ & $\begin{array}{c}\text { Static } \\
\text { Cone } \\
\text { Penetrometer } \\
(\mathrm{mm})\end{array}$ \\
\hline $02: 50 P M$ & $\bar{F}$ & 1.54 & & & \\
\hline 03:00 PM & $\bar{F}$ & $\overline{1.54}$ & Infinite & & \\
\hline 03:10 PM & $\bar{F}$ & 1.54 & & & \\
\hline & & & & & \\
\hline & & & & & \\
\hline & & & & & \\
\hline & & & & & \\
\hline
\end{tabular}

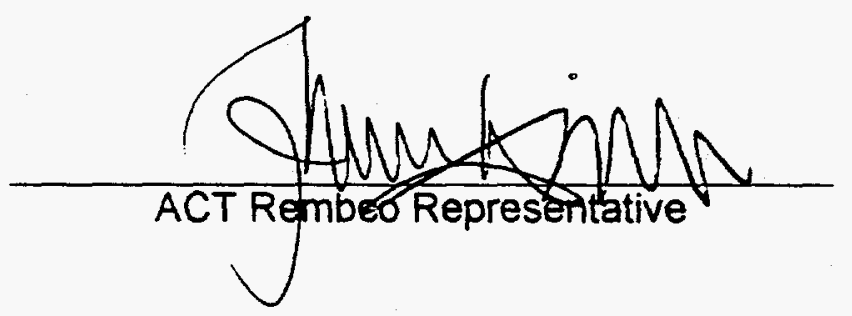




\section{ACT REMBCO JOINT VENTURE \\ Ornl Wag 4 Seeps 4 \& 6 Removal Action Project}

Quality Contol Report

October 14, 1996

\begin{tabular}{|c|c|c|c|c|c|}
\hline Time of Test & $\begin{array}{c}\text { Mix } \\
\text { Identificaton }\end{array}$ & $\begin{array}{c}\text { Specific } \\
\text { Gravity } \\
\text { (Kg/Litre) }\end{array}$ & $\begin{array}{l}\text { Marsh } \\
\text { Funnel } \\
\text { (seconds) }\end{array}$ & $\begin{array}{l}\text { Coefficient } \\
\text { Pressure } \\
\text { Filtration } \\
\text { (minutes-1/2) }\end{array}$ & $\begin{array}{c}\text { Static } \\
\text { Cone } \\
\text { Penetrometer } \\
(\mathrm{mm})\end{array}$ \\
\hline $07: 50 \mathrm{AM}$ & $M^{\prime \prime}$ & 1.23 & 44 & Microcem $B$ & \\
\hline 08:00 AM & $M^{\prime \prime}$ & 1.22 & & & \\
\hline $08: 10 \mathrm{AM}$ & $M^{\prime \prime}$ & 1.25 & 47 & & \\
\hline $08: 20 \mathrm{AM}$ & $M^{\prime \prime}$ & 1.26 & & & \\
\hline $08: 30 \mathrm{AM}$ & M" & 1.23 & 45 & & \\
\hline $08: 40 \mathrm{AM}$ & $M^{\prime \prime}$ & 1.24 & & & \\
\hline $08: 50$ AM & M" & 1.23 & 49 & & \\
\hline 09:00 AM & M" & 1.22 & & & \\
\hline $09: 10 \mathrm{AM}$ & $M^{\prime \prime}$ & 1.23 & 48 & & \\
\hline $09: 20 \mathrm{AM}$ & $M^{\prime \prime}$ & 1.24 & & & \\
\hline $09: 30 \mathrm{AM}$ & $M^{\prime \prime}$ & 1.26 & 49 & & \\
\hline $09: 40$ AM & $M^{\prime \prime}$ & 1.23 & & & \\
\hline $09: 50 \mathrm{AM}$ & $M^{\prime \prime}$ & 1.23 & 47 & & \\
\hline $10: 14 \mathrm{AM}$ & $F$ & 1.50 & 89 & & \\
\hline $10: 25 \mathrm{AM}$ & $\bar{F}$ & 1.52 & & & \\
\hline $10: 35 \mathrm{AM}$ & $\bar{F}$ & 1.53 & 94 & & \\
\hline $10: 45 \mathrm{AM}$ & $F$ & 1.53 & & & \\
\hline $11: 00 \mathrm{AM}$ & $F$ & 1.53 & 98 & & \\
\hline $11: 10 \mathrm{AM}$ & $F$ & 1.54 & & & \\
\hline $11: 20 \mathrm{AM}$ & $F$ & 1.53 & 101 & & \\
\hline $11: 30 \mathrm{AM}$ & $F$ & 1.53 & & & \\
\hline 11:40 AM & $F$ & 1.54 & 105 & & \\
\hline $12: 55 \mathrm{PM}$ & $F$ & 1.53 & & & \\
\hline $01: 10 \mathrm{PM}$ & $\bar{F}$ & 1.53 & 122 & & \\
\hline $01: 20 \mathrm{PM}$ & $\bar{F}$ & 1.52 & & & \\
\hline $01: 30 \mathrm{PM}$ & $\bar{F}$ & 1.53 & 107 & & \\
\hline
\end{tabular}




\section{ACT REMBCO JOINT VENTURE \\ Ornl Wag 4 Seeps 4 \& 6 Removal Action Project \\ Quality Contol Report \\ October 14, 1996}

\begin{tabular}{|c|c|c|c|c|c|}
\hline Time of Test & $\begin{array}{c}\text { Mix } \\
\text { Identificaton }\end{array}$ & $\begin{array}{c}\text { Specific } \\
\text { Gravity } \\
\text { (Kg/Litre) }\end{array}$ & $\begin{array}{c}\text { Marsh } \\
\text { Funnel } \\
\text { (seconds) }\end{array}$ & $\begin{array}{c}\text { Coefficient } \\
\text { Pressure } \\
\text { Filtration } \\
\text { (minutes-1/2) }\end{array}$ & $\begin{array}{c}\text { Static } \\
\text { Cone } \\
\text { Penetrometer } \\
\text { (mm) }\end{array}$ \\
\hline $01: 40 \mathrm{PM}$ & $\mathrm{F}$ & 1.53 & & & \\
\hline $01: 50 \mathrm{PM}$ & $\mathrm{F}$ & 1.53 & 99 & & \\
\hline $02: 00 \mathrm{PM}$ & $\mathrm{F}$ & 1.53 & & & \\
\hline $02: 10 \mathrm{PM}$ & $\mathrm{F}$ & 1.53 & 104 & & \\
\hline $02: 20 \mathrm{PM}$ & $\mathrm{F}$ & 1.54 & & & \\
\hline $02: 30 \mathrm{PM}$ & $\mathrm{F}$ & 1.53 & 119 & & \\
\hline $02: 40 \mathrm{PM}$ & $\mathrm{F}$ & 1.53 & & & \\
\hline $02: 50 \mathrm{PM}$ & $\mathrm{F}$ & 1.53 & 109 & & \\
\hline $03: 00 \mathrm{PM}$ & $\mathrm{F}$ & 1.53 & & & \\
\hline $03: 10 \mathrm{PM}$ & $\mathrm{F}$ & 1.53 & 107 & & \\
\hline $03: 20 \mathrm{PM}$ & $\mathrm{F}$ & 1.52 & & & \\
\hline $03: 30 \mathrm{PM}$ & $\mathrm{F}$ & 1.53 & 114 & & \\
\hline $03: 40 \mathrm{PM}$ & $\mathrm{F}$ & 1.53 & & & \\
\hline $03: 50 \mathrm{PM}$ & $\mathrm{F}$ & 1.53 & 100 & & \\
\hline $04: 00 \mathrm{PM}$ & F & 1.51 & & & \\
\hline $04: 10 \mathrm{PM}$ & $\mathrm{F}$ & 1.53 & 110 & & \\
\hline $04: 20 \mathrm{PM}$ & $\mathrm{F}$ & 1.52 & & & \\
\hline & & & & & \\
\hline
\end{tabular}

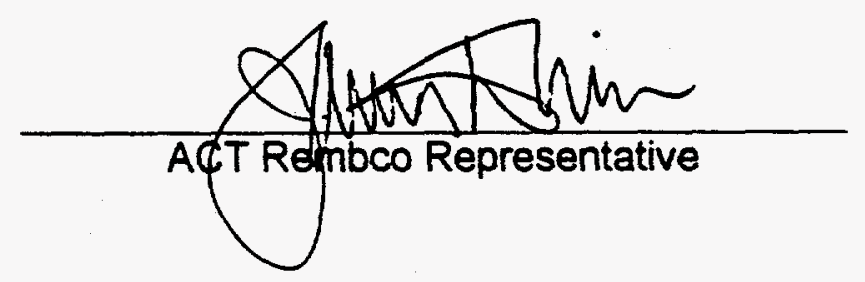




\section{ACT REMBCO JOINT VENTURE \\ Ornl Wag 4 Seeps 4 \& 6 Removal Action Project}

Quality Contol Report

October 15, 1996

\begin{tabular}{|c|c|c|c|c|c|}
\hline Time of Test & $\begin{array}{c}\text { Mix } \\
\text { identificaton }\end{array}$ & $\begin{array}{c}\text { Specific } \\
\text { Gravity } \\
\text { (Kg/Litre) }\end{array}$ & $\begin{array}{c}\text { Marsh } \\
\text { Funnel } \\
\text { (seconds) }\end{array}$ & $\begin{array}{c}\text { Coefficient } \\
\text { Pressure } \\
\text { Filtration } \\
\text { (minutes-1/2) }\end{array}$ & $\begin{array}{c}\text { Static } \\
\text { Cone } \\
\text { Penetrometer } \\
(\mathrm{mm})\end{array}$ \\
\hline 08:00 AM & $F$ & 1.52 & Infinite & & \\
\hline $08: 10 \mathrm{AM}$ & $F$ & 1.53 & & & \\
\hline $08: 20 \mathrm{AM}$ & $F$ & 1.53 & Infinite & & \\
\hline $08: 30 \mathrm{AM}$ & $F$ & 1.53 & & & \\
\hline $08: 40 \mathrm{AM}$ & $\bar{F}$ & 1.53 & Infinite & & \\
\hline $08: 50 \mathrm{AM}$ & $F$ & 1.53 & & & \\
\hline $09: 00 \mathrm{AM}$ & $\bar{F}$ & 1.54 & Infinite & & \\
\hline $09: 10 \mathrm{AM}$ & $\bar{F}$ & 1.54 & & & \\
\hline $09: 20 \mathrm{AM}$ & $\bar{F}$ & 1.53 & Infinite & & \\
\hline $09: 30 \mathrm{AM}$ & $\bar{F}$ & 1.55 & & & \\
\hline $09: 40 \mathrm{AM}$ & $F$ & 1.54 & Infinite & & \\
\hline $09: 50 \mathrm{AM}$ & $F$ & 1.54 & & & \\
\hline $10: 00 \mathrm{AM}$ & $\bar{F}$ & 1.54 & Infinite & & \\
\hline 10:10 AM & $\bar{F}$ & 1.54 & & & \\
\hline $10: 20 \mathrm{AM}$ & $\bar{F}$ & 1.54 & 127 & & \\
\hline $10: 30 \mathrm{AM}$ & $\bar{F}$ & 1.54 & & & \\
\hline $10: 40 \mathrm{AM}$ & $F$ & 1.54 & Infinite & & \\
\hline $10: 50 \mathrm{AM}$ & $F$ & 1.54 & & & \\
\hline $11: 00 \mathrm{AM}$ & $\bar{F}$ & 1.55 & 131 & & \\
\hline $11: 10 \mathrm{AM}$ & $F$ & 1.54 & & & \\
\hline $11: 20 \mathrm{AM}$ & $F$ & 1.54 & Infinite & & \\
\hline 11:30 AM & $F$ & 1.53 & & & \\
\hline $11: 40 \mathrm{AM}$ & $F$ & 1.53 & 121 & & \\
\hline $11: 50 \mathrm{AM}$ & $\bar{F}$ & 1.53 & & & \\
\hline $12: 45 \mathrm{PM}$ & $\bar{F}$ & 1.54 & 115 & & \\
\hline $12: 55 \mathrm{PM}$ & $F$ & 1.54 & & & \\
\hline
\end{tabular}




\section{ACT REMBCO JOINT VENTURE \\ Ornl Wag 4 Seeps 4 \& 6 Removal Action Project}

Quality Contol Report

October 15, 1996

\begin{tabular}{|c|c|c|c|c|c|}
\hline Time of Test & $\begin{array}{c}\text { Mix } \\
\text { Identificaton }\end{array}$ & $\begin{array}{c}\text { Specific } \\
\text { Gravity } \\
\text { (Kg/Litre) }\end{array}$ & $\begin{array}{c}\text { Marsh } \\
\text { Funnel } \\
\text { (seconds) }\end{array}$ & $\begin{array}{c}\text { Coefficient } \\
\text { Pressure } \\
\text { Filtration } \\
\text { (minutes-1/2) }\end{array}$ & $\begin{array}{c}\text { Static } \\
\text { Cone } \\
\text { Penetrometer } \\
\text { (mm) }\end{array}$ \\
\hline $01: 05 \mathrm{PM}$ & $\mathrm{F}$ & 1.53 & 118 & & \\
\hline $01: 15 \mathrm{PM}$ & $\mathrm{F}$ & 1.53 & & & \\
\hline $01: 40 \mathrm{PM}$ & $\mathrm{F}$ & 1.52 & 115 & & \\
\hline $01: 50 \mathrm{PM}$ & $\mathrm{F}$ & 1.53 & & & \\
\hline $02: 00 \mathrm{PM}$ & $\mathrm{F}$ & 1.53 & 109 & & \\
\hline $02: 10 \mathrm{PM}$ & $\mathrm{F}$ & 1.52 & & & \\
\hline $02: 20 \mathrm{PM}$ & $\mathrm{F}$ & 1.53 & 110 & & \\
\hline $02: 30 \mathrm{PM}$ & $\mathrm{F}$ & 1.53 & 113 & & \\
\hline $02: 40 \mathrm{PM}$ & $\mathrm{F}$ & 1.52 & & & \\
\hline $02: 50 \mathrm{PM}$ & $\mathrm{F}$ & 1.53 & 114 & & \\
\hline $03: 00 \mathrm{PM}$ & $\mathrm{F}$ & 1.53 & & & \\
\hline $03: 10 \mathrm{PM}$ & $\mathrm{F}$ & 1.54 & 108 & & \\
\hline $03: 20 \mathrm{PM}$ & $\mathrm{F}$ & 1.53 & & & \\
\hline $03: 30 \mathrm{PM}$ & $\mathrm{F}$ & 1.53 & 116 & & \\
\hline $03: 40 \mathrm{PM}$ & $\mathrm{F}$ & 1.54 & & & \\
\hline $03: 50 \mathrm{PM}$ & $\mathrm{F}$ & 1.54 & 122 & & \\
\hline $04: 00 \mathrm{PM}$ & $\mathrm{F}$ & 1.54 & & & \\
\hline $04: 10 \mathrm{PM}$ & $\mathrm{F}$ & 1.54 & Infinite & & \\
\hline $04: 20 \mathrm{PM}$ & $\mathrm{F}$ & 1.54 & & & \\
\hline $04: 30 \mathrm{PM}$ & $\mathrm{F}$ & 1.54 & Infinite & & \\
\hline
\end{tabular}




\section{ACT REMBCO JOINT VENTURE Ornl Wag 4 Seeps 4 \& 6 Removal Action Project}

Quality Contol Report

October 16, 1996

\begin{tabular}{|c|c|c|c|c|c|}
\hline Time of Test & $\begin{array}{c}\text { Mix } \\
\text { Identificaton }\end{array}$ & $\begin{array}{c}\text { Specific } \\
\text { Gravity } \\
\text { (Kg/Litre) }\end{array}$ & $\begin{array}{c}\text { Marsh } \\
\text { Funnel } \\
\text { (seconds) }\end{array}$ & $\begin{array}{c}\text { Coefficient } \\
\text { Pressure } \\
\text { Filtration } \\
\text { (minutes-1/2) }\end{array}$ & $\begin{array}{c}\text { Static } \\
\text { Cone } \\
\text { Penetrometer } \\
(\mathrm{mm})\end{array}$ \\
\hline $08: 00 \mathrm{AM}$ & $\bar{F}$ & 1.50 & 105 & & \\
\hline $08: 10 \mathrm{AM}$ & $F$ & 1.52 & & & \\
\hline $08: 20 \mathrm{AM}$ & $F$ & 1.53 & 108 & & \\
\hline $08: 30 \mathrm{AM}$ & $F$ & 1.53 & & & \\
\hline $08: 40 \mathrm{AM}$ & $\bar{F}$ & 1.53 & 107 & & \\
\hline $08: 50 \mathrm{AM}$ & $F$ & 1.53 & & & \\
\hline 09:00 AM & $F$ & 1.53 & 113 & & \\
\hline $09: 10 \mathrm{AM}$ & $F$ & 1.53 & & & \\
\hline $09: 20 \mathrm{AM}$ & $\bar{F}$ & 1.53 & 112 & & \\
\hline $09: 30 \mathrm{AM}$ & $\bar{F}$ & 1.53 & & & \\
\hline $09: 40 \mathrm{AM}$ & $F$ & 1.53 & 115 & & \\
\hline 09:50 AM & $F$ & 1.53 & & & \\
\hline $10: 05 \mathrm{AM}$ & $F$ & 1.54 & 125 & & \\
\hline $11: 10 \mathrm{AM}$ & $M^{\prime \prime}$ & 1.25 & 45 & & \\
\hline $11: 20 \mathrm{AM}$ & $M^{\prime \prime}$ & 1.24 & & & \\
\hline $11: 30 \mathrm{AM}$ & M" & 1.25 & 46 & & \\
\hline $11: 40$ AM & $M^{\prime \prime}$ & 1.24 & & & \\
\hline $12: 35 \mathrm{PM}$ & $M^{\prime \prime}$ & 1.24 & $\overline{43}$ & & \\
\hline $12: 45 \mathrm{PM}$ & $M^{\prime \prime}$ & 1.24 & & & \\
\hline $12: 55 \mathrm{PM}$ & $M^{\prime \prime}$ & 1.24 & 44 & & \\
\hline 01:05 PM & M" & 1.23 & & & \\
\hline $01: 15 \mathrm{PM}$ & $M^{\prime \prime}$ & 1.24 & 44 & & \\
\hline $01: 25 \mathrm{PM}$ & $M^{\prime \prime}$ & 1.24 & & & \\
\hline $01: 35$ PM & $M^{\prime \prime}$ & 1.23 & 46 & & \\
\hline
\end{tabular}




\section{ACT REMBCO JOINT VENTURE \\ Ornl Wag 4 Seeps 4 \& 6 Removal Action Project}

Quality Contol Report

October 18, 1996

\begin{tabular}{|c|c|c|c|c|c|}
\hline Time of Test & $\begin{array}{c}\text { Mix } \\
\text { Identificaton }\end{array}$ & $\begin{array}{c}\text { Specific } \\
\text { Gravity } \\
\text { (Kg/Litre) }\end{array}$ & $\begin{array}{c}\text { Marsh } \\
\text { Funnel } \\
\text { (seconds) }\end{array}$ & $\begin{array}{c}\text { Coefficient } \\
\text { Pressure } \\
\text { Filtration } \\
\text { (minutes-1/2) }\end{array}$ & $\begin{array}{c}\text { Static } \\
\text { Cone } \\
\text { Penetrometer } \\
\text { (mm) }\end{array}$ \\
\hline $07: 48 \mathrm{AM}$ & $\mathrm{M}^{\prime \prime}$ & 1.25 & 48 & & \\
\hline $07: 58 \mathrm{AM}$ & $\mathrm{M}^{\prime \prime}$ & 1.26 & 51 & & \\
\hline $08: 08 \mathrm{AM}$ & $\mathrm{M}^{\prime \prime}$ & 1.33 & Infinite & & \\
\hline $08: 18 \mathrm{AM}$ & $\mathrm{M}^{\prime \prime}$ & 1.34 & Infinite & & \\
\hline $08: 28 \mathrm{AM}$ & $\mathrm{M}^{\prime \prime}$ & 1.33 & Infinite & & \\
\hline $09: 15 \mathrm{AM}$ & $\mathrm{M}^{\prime \prime}$ & 1.24 & 48 & & \\
\hline $09: 25 \mathrm{AM}$ & $\mathrm{M}^{\prime \prime}$ & 1.23 & 47 & & \\
\hline $09: 35 \mathrm{AM}$ & $\mathrm{M}^{\prime \prime}$ & 1.24 & & & \\
\hline $09: 45 \mathrm{AM}$ & $\mathrm{M}^{\prime \prime}$ & 1.23 & 45 & & \\
\hline $09: 55 \mathrm{AM}$ & $\mathrm{M}^{\prime \prime}$ & 1.23 & & & \\
\hline $10: 05 \mathrm{AM}$ & $\mathrm{M}^{\prime \prime}$ & 1.23 & 46 & & \\
\hline $10: 15 \mathrm{AM}$ & $\mathrm{M}^{\prime \prime}$ & 1.23 & & & \\
\hline $10: 25 \mathrm{AM}$ & $\mathrm{M}^{\prime \prime}$ & 1.23 & 45 & & \\
\hline $10: 35 \mathrm{AM}$ & $\mathrm{M}^{\prime \prime}$ & 1.24 & & & \\
\hline $10: 45 \mathrm{AM}$ & $\mathrm{M}^{\prime \prime}$ & 1.23 & 44 & & \\
\hline $10: 55 \mathrm{AM}$ & $\mathrm{M}^{\prime \prime}$ & 1.23 & & & \\
\hline $11: 05 \mathrm{AM}$ & $\mathrm{M}^{\prime \prime}$ & 1.23 & 46 & & \\
\hline $11: 15 \mathrm{AM}$ & $\mathrm{M}^{\prime \prime}$ & 1.23 & & & \\
\hline $11: 25 \mathrm{AM}$ & $\mathrm{M}^{\prime \prime}$ & 1.24 & 45 & & \\
\hline $11: 35 \mathrm{AM}$ & $\mathrm{M}^{\prime \prime}$ & 1.24 & & & \\
\hline $11: 45 \mathrm{AM}$ & $\mathrm{M}^{\prime \prime}$ & 1.23 & 44 & & \\
\hline $12: 40 \mathrm{PM}$ & $\mathrm{M}^{\prime \prime}$ & 1.23 & & & \\
\hline
\end{tabular}

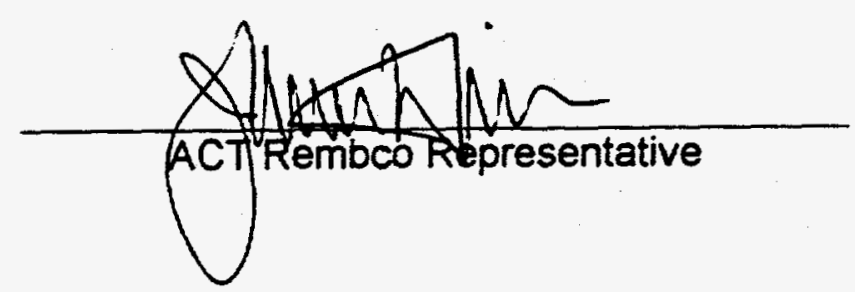



ACRYLAMIDE GROUT

QUALITY CONTROL TESTING REPORTS 
ACT REMBCO JOINT VENTURE

Ornl Wag 4 Seeps 4 \& 6 Removal Action Project

Acrylamide Testing Record For

September 06, 1996

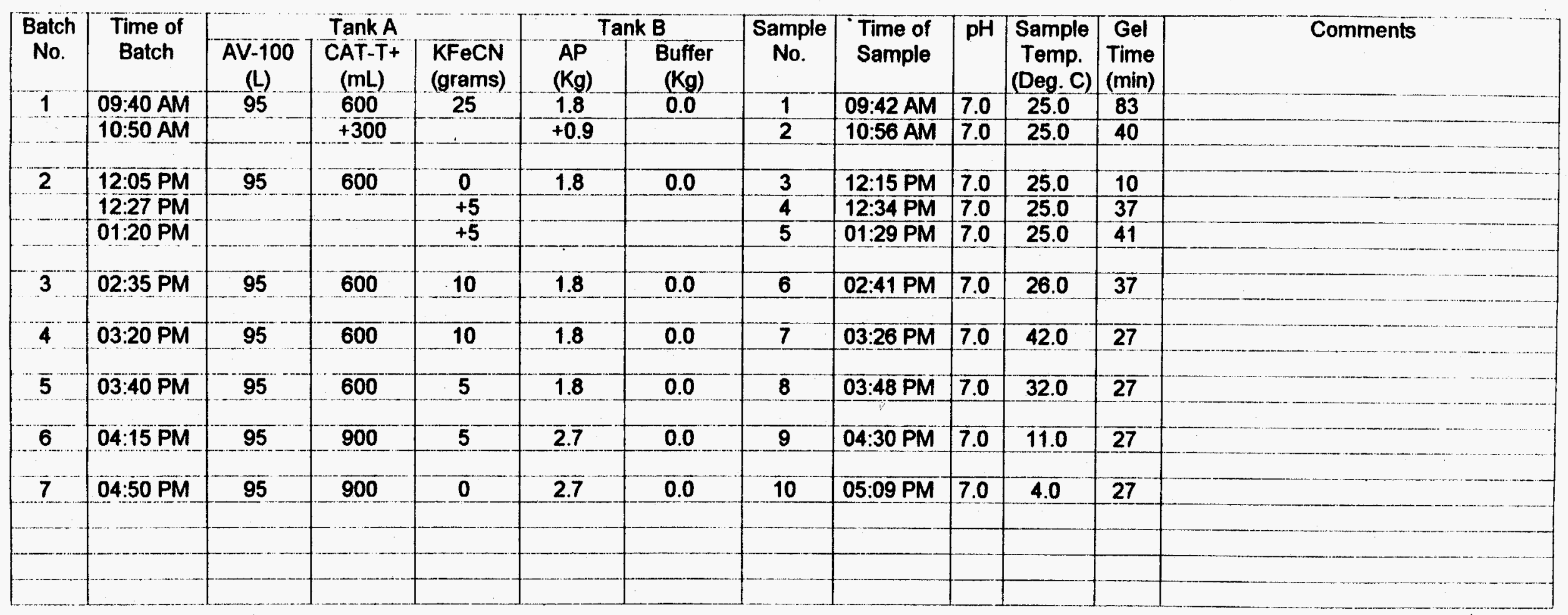

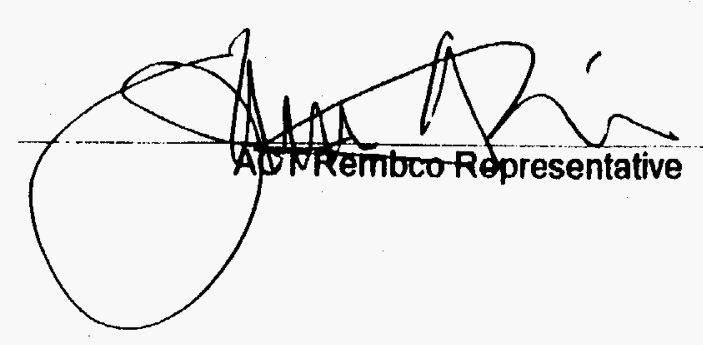


ACT REMBCO JOINT VENTURE

Ornl Wag 4 Seeps 4 \& 6 Removal Action Project

Acrylamide Batching Record For

September 17, 1996

\begin{tabular}{|c|c|c|c|c|c|c|c|c|c|c|c|c|}
\hline \multirow{2}{*}{$\begin{array}{l}\text { Batch } \\
\text { No. }\end{array}$} & \multirow{2}{*}{$\begin{array}{l}\text { Time of } \\
\text { Batch }\end{array}$} & \multicolumn{3}{|c|}{ Tank A } & \multicolumn{2}{|c|}{ Tank B } & \multirow{2}{*}{$\begin{array}{c}\text { Sample } \\
\text { No. }\end{array}$} & \multirow{2}{*}{$\begin{array}{l}\text { Time of } \\
\text { Sample }\end{array}$} & \multirow[t]{2}{*}{$\mathrm{pH}$} & \multirow{2}{*}{$\begin{array}{c}\text { Sample } \\
\text { Temp. } \\
\text { (Deg. C) }\end{array}$} & \multirow{2}{*}{$\begin{array}{c}\text { Gel } \\
\text { Time } \\
\text { (min) }\end{array}$} & \multirow[t]{2}{*}{ Comments } \\
\hline & & $\begin{array}{c}\mathrm{AV}-100 \\
\text { (L) }\end{array}$ & $\begin{array}{c}\text { CAT-T+ } \\
(\mathrm{mL})\end{array}$ & $\begin{array}{l}\mathrm{KFeCN} \\
\text { (grams) }\end{array}$ & $\begin{array}{c}\mathrm{AP} \\
(\mathrm{Kg})\end{array}$ & $\begin{array}{c}\text { Buffer } \\
\text { (Kg) }\end{array}$ & & & & & & \\
\hline \multirow{3}{*}{1} & 09:00 AM & 95 & 600 & 5 & 1.8 & 0.0 & 1 & $09: 05 \mathrm{AM}$ & 7.5 & 25.0 & 14 & \\
\hline & $09.25 \mathrm{AM}$ & & & +5 & & & 2 & $09: 28 \mathrm{AM}$ & 7.5 & 25.0 & 43 & \\
\hline & & & & & & & & & & & & \\
\hline \multirow[t]{2}{*}{2} & $10: 30 \mathrm{AM}$ & 95 & 600 & 5 & 1.8 & 0.0 & 3 & 10:34 AM & 7.5 & 25.0 & 14 & \\
\hline & & & & & & & & & & & & \\
\hline \multirow[t]{2}{*}{3} & $11: 00 \mathrm{AM}$ & 95 & 600 & o & 1.8 & 0.0 & 4 & 11:06 AM & 7.5 & 26.0 & 6 & \\
\hline & & & & & & & & & & & & \\
\hline 4 & 11:25 AM & 95 & 600 & 0 & 1.8 & 0.0 & 5 & 11:29 AM & 7.5 & 26.0 & 7 & \\
\hline \multirow[t]{2}{*}{5} & $12: 00 \mathrm{PM}$ & 95 & 600 & 0 & 1.8 & 0.0 & 6 & $12: 03 \mathrm{PM}$ & 7.5 & 26.0 & 6 & \\
\hline & & & & & & & & & & & & \\
\hline 6 & 12:35 PM & 95 & 600 & 0 & 1.8 & 0.0 & 7 & $12: 37 \mathrm{PM}$ & 7.5 & 26.0 & 6 & \\
\hline \multirow[t]{2}{*}{7} & 01:00 PM & 95 & 600 & 0 & 1.8 & 0.0 & 8 & $01: 02 \mathrm{PM}$ & 7.5 & 26.0 & 6 & \\
\hline & & & & & & & & & & & & \\
\hline \multirow[t]{3}{*}{8} & $01: 25$ PM & 95 & 600 & 0 & 1.8 & 0.0 & 9 & $01: 29 \mathrm{PM}$ & 7.5 & 26.0 & 6 & \\
\hline & & & & +5 & & & 10 & $01: 50 \mathrm{PM}$ & 7.5 & 26.0 & 13 & \\
\hline & & & & & & & & & & & & \\
\hline \multirow{2}{*}{9} & 02:55 PM & 95 & 600 & 0 & 1.8 & 0.0 & 11 & $02.57 \mathrm{PM}$ & 7.5 & 26.0 & 12 & \\
\hline & & & & & & & & & & & & \\
\hline \multirow{2}{*}{10} & $03: 25$ PM & 95 & 600 & 0 & 1.8 & 0.0 & 12 & 03:30 PM & 7.5 & 26.0 & 14 & \\
\hline & & & & & & & & & & & & \\
\hline
\end{tabular}


ACT REMBCO JOINT VENTURE

Ornl Wag 4 Seeps 4 \& 6 Removal Action Project

Acrylamide Batching Record For

September 18, 1996

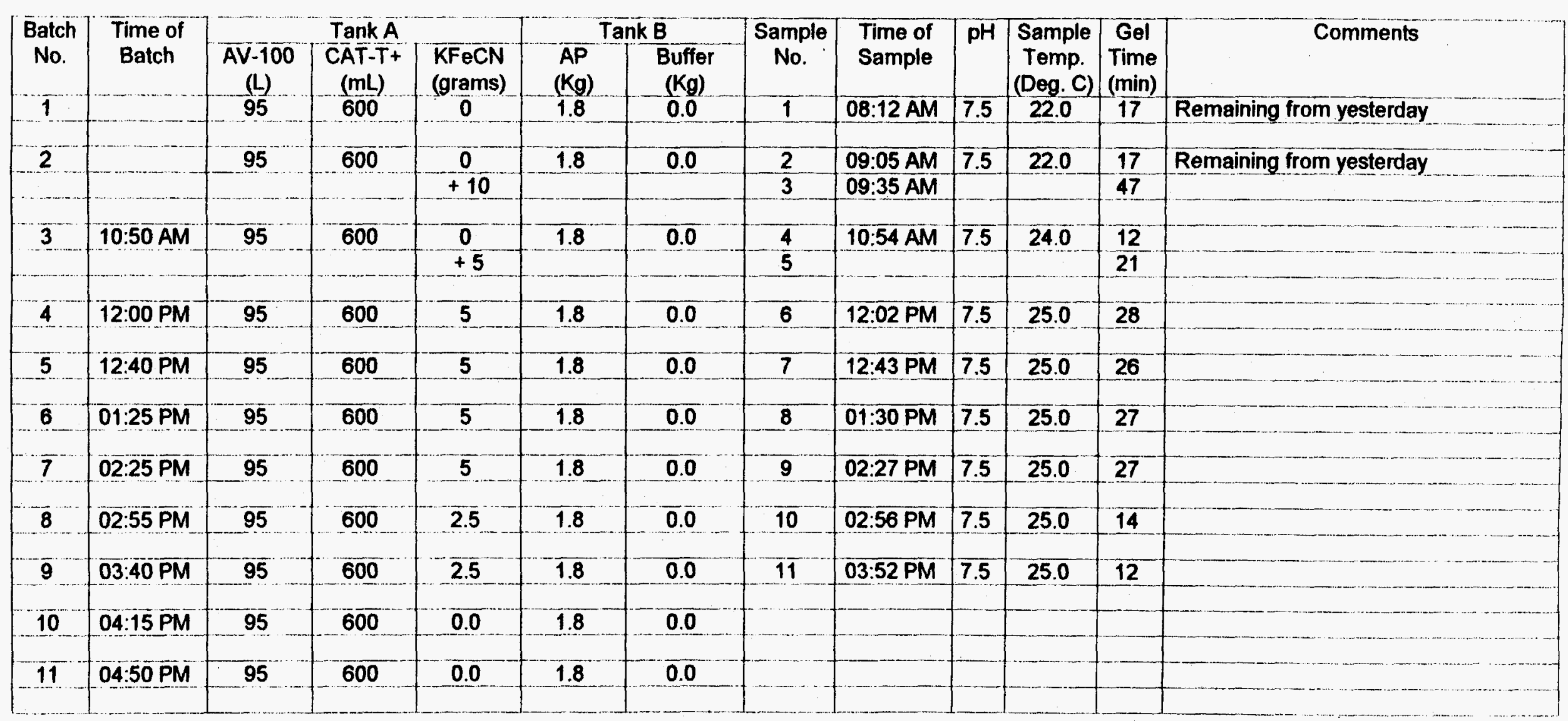


ACT REMBCO JOINT VENTURE

Ornl Wag 4 Seeps 4 \& 6 Removal Action Project

Acrylamide Batching Record For

September 19, 1996

\begin{tabular}{|c|c|c|c|c|c|c|c|c|c|c|c|c|}
\hline \multirow{2}{*}{$\begin{array}{c}\text { Batch } \\
\text { No. }\end{array}$} & \multirow{2}{*}{$\begin{array}{l}\text { Time of } \\
\text { Batch }\end{array}$} & \multicolumn{3}{|c|}{ Tank A } & \multicolumn{2}{|c|}{ Tank B } & \multirow{2}{*}{$\begin{array}{c}\text { Sample } \\
\text { No. }\end{array}$} & \multirow{2}{*}{$\begin{array}{l}\text { Time of } \\
\text { Sample }\end{array}$} & \multirow[t]{2}{*}{$\mathrm{pH}$} & \multirow{2}{*}{$\begin{array}{c}\text { Sample } \\
\text { Temp. } \\
\text { (Deg. C) }\end{array}$} & \multirow{2}{*}{$\begin{array}{l}\text { Gel } \\
\text { Time } \\
\text { (min) }\end{array}$} & \multirow[t]{2}{*}{ Comments } \\
\hline & & $\begin{array}{c}A V-100 \\
\text { (L) }\end{array}$ & $\begin{array}{c}\text { CAT-T+ } \\
(\mathrm{mL})\end{array}$ & $\begin{array}{l}\text { KFeCN } \\
\text { (grams) }\end{array}$ & $\begin{array}{l}\mathrm{AP} \\
(\mathrm{Kg})\end{array}$ & $\begin{array}{l}\text { Buffer } \\
(\mathrm{Kg})\end{array}$ & & & & & & \\
\hline 1 & & 95 & 600 & 0 & 1.8 & 0.0 & 1 & $12: 25 \mathrm{PM}$ & 7.5 & 19.0 & 12 & Remaining from yesterday \\
\hline 2 & & 95 & 600 & 0 & 1.8 & 0.0 & 2 & $01: 02 \mathrm{PM}$ & 7.5 & 19.0 & 12 & Remaining from yesterday \\
\hline 3 & $01: 43 \mathrm{PM}$ & 95 & 600 & 0 & 1.8 & 0.0 & 3 & 01:46 PM & 7.5 & 22.0 & 10 & \\
\hline & & & & & & & & & & & & \\
\hline
\end{tabular}

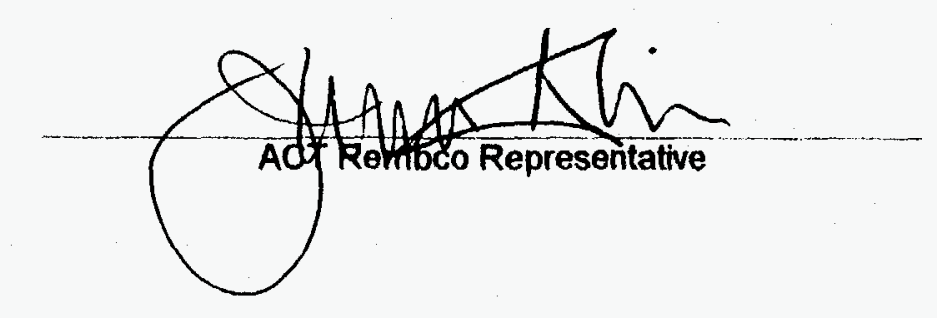




\section{ACT REMBCO JOINT VENTURE}

Ornl Wag 4 Seeps 4 \& 6 Removal Action Project

Acrylamide Batching Record For

September 25, 1996

\begin{tabular}{|c|c|c|c|c|c|c|c|c|c|c|c|c|}
\hline \multirow{2}{*}{$\begin{array}{l}\text { Batch } \\
\text { No. }\end{array}$} & \multirow{2}{*}{$\begin{array}{l}\text { Time of } \\
\text { Batch }\end{array}$} & \multicolumn{3}{|c|}{ Tank A } & \multicolumn{2}{|c|}{ Tank $\mathrm{B}$} & \multirow{2}{*}{$\begin{array}{c}\text { Sample } \\
\text { No. }\end{array}$} & \multirow{2}{*}{$\begin{array}{l}\text { Time of } \\
\text { Sample }\end{array}$} & \multirow[t]{2}{*}{$\mathrm{pH}$} & \multirow{2}{*}{$\begin{array}{c}\text { Sample } \\
\text { Temp. } \\
\text { (Deg. C) }\end{array}$} & \multirow{2}{*}{$\begin{array}{c}\text { Gel } \\
\text { Time } \\
\text { (min) }\end{array}$} & \multirow[t]{2}{*}{ Comments } \\
\hline & & $\begin{array}{c}A V-100 \\
\text { (L) }\end{array}$ & $\begin{array}{c}\text { CAT-T+ } \\
(\mathrm{mL})\end{array}$ & $\begin{array}{l}\mathrm{KFeCN} \\
\text { (grams) }\end{array}$ & $\begin{array}{c}\text { AP } \\
(\mathrm{Kg})\end{array}$ & $\begin{array}{c}\text { Buffer } \\
\text { (Kg) }\end{array}$ & & & & & & \\
\hline 1 & & 95 & 600 & 25 & 1.8 & 0.0 & 1 & 07:40 AM & 7.5 & 21.5 & 83 & Remaining from yesterday \\
\hline 2 & 08:00 AM & 95 & 600 & 10 & 1.8 & 0.0 & 2 & 08:05 AM & 7.5 & 21.5 & 45 & \\
\hline 3 & 09:10 AM & 95 & 600 & 5 & 1.8 & 0.0 & 3 & $09: 18 \mathrm{AM}$ & 7.5 & 21.5 & 39 & \\
\hline 4 & $10: 10 \mathrm{AM}$ & 95 & 600 & 0 & 1.8 & 0.0 & 4 & $10: 25 \mathrm{AM}$ & 7.5 & 22.0 & 10 & \\
\hline & & & $-\ldots$ & +10 & & & 5 & $10.57 \mathrm{AM}$ & & & 37 & \\
\hline 5 & $01: 20 \mathrm{PM}$ & 95 & 600 & 10 & 1.8 & 0.0 & 6 & $01: 30 \mathrm{PM}$ & 7.5 & 24.0 & 35 & \\
\hline 6 & $02: 30 \mathrm{PM}$ & 95 & 600 & 10 & 1.8 & 0.0 & 7 & $02: 43 \overline{P M}$ & 7.5 & 24.0 & 28 & \\
\hline 7 & $03: 30 \mathrm{PM}$ & 95 & 600 & 10 & $1 . \overline{8}$ & 0.0 & 8 & $03: 35 \mathrm{PM}$ & 7.5 & 24.0 & 48 & \\
\hline & $\cdots$ & & & & & & & & & & & \\
\hline
\end{tabular}

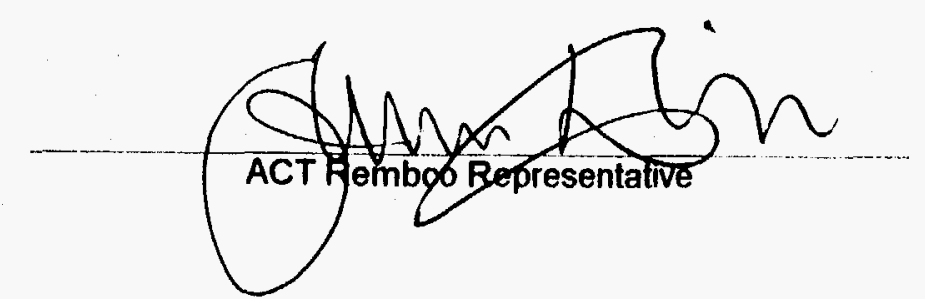


ACT REMBCO JOINT VENTURE

Ornl Wag 4 Seeps 4 \& 6 Removal Action Project

Acrylamide Batching Record For

September 26, 1996

\begin{tabular}{|c|c|c|c|c|c|c|c|c|c|c|c|c|}
\hline \multirow{2}{*}{$\begin{array}{l}\text { Batch } \\
\text { No. }\end{array}$} & \multirow{2}{*}{$\begin{array}{l}\text { Time of } \\
\text { Batch }\end{array}$} & \multicolumn{3}{|c|}{$\operatorname{Tank} \bar{A}$} & \multicolumn{2}{|c|}{ Tank B } & \multirow{2}{*}{$\begin{array}{c}\text { Sample } \\
\text { No. }\end{array}$} & \multirow{2}{*}{$\begin{array}{l}\text { Time of } \\
\text { Sample }\end{array}$} & \multirow[t]{2}{*}{$\mathrm{pH}$} & \multirow{2}{*}{$\begin{array}{l}\text { Sample } \\
\text { Temp. } \\
\text { (Deg. C) }\end{array}$} & \multirow{2}{*}{$\begin{array}{c}\text { Gel } \\
\text { Time } \\
\text { (min) }\end{array}$} & \multirow[t]{2}{*}{ Comments } \\
\hline & & $\begin{array}{l}A \bar{V}-100 \\
\text { (L) }\end{array}$ & $\begin{array}{c}\text { CAT-T+ } \\
(\mathrm{mL})\end{array}$ & $\begin{array}{l}\mathrm{KFeCN} \\
\text { (grams) }\end{array}$ & $\begin{array}{c}\bar{A} \bar{P} \\
(\mathrm{Kg})\end{array}$ & $\begin{array}{c}\text { Buffer } \\
\text { (Kg) }\end{array}$ & & & & & & \\
\hline 1 & & 95 & 600 & 10 & 1.8 & 0.0 & & & 7.5 & & & Remaining from yesterday \\
\hline 2 & & 95 & 600 & 10 & 1.8 & 0.0 & 1 & $07: 37$ AM & 7.5 & 21.0 & 40 & Remaining from yesterday \\
\hline$\overline{3}$ & $08: \overline{45} \mathrm{AM}$ & 95 & 600 & 10 & 1.8 & 0.0 & 2 & 08:49 AM & 7.5 & 21.5 & 38 & \\
\hline & & & & & & & & & & & & \\
\hline 4 & $09: 20 \mathrm{AM}$ & 95 & 600 & 10 & 1.8 & 0.0 & 3 & $09: 25$ AM & 7.5 & 22.0 & 40 & \\
\hline 5 & $10: 50 \mathrm{AM}$ & 95 & 600 & 10 & 1.8 & 0.0 & 4 & $10.54 \mathrm{AM}$ & 7.5 & 22.0 & 40 & \\
\hline & & & & & & & & & & & & \\
\hline$\overline{6}$ & $11: 30 \mathrm{AM}$ & 95 & 600 & 10 & 1.8 & 0.0 & 5 & $11: 40$ AM & 7.5 & 22.0 & 42 & \\
\hline 7 & $1225 \overline{P M}$ & 95 & 600 & 10 & 1.8 & 0.0 & 6 & $12: 32 \mathrm{PM}$ & 7.5 & 22.0 & 34 & \\
\hline & & & & & & & & & & & & \\
\hline 8 & $01: 10 \mathrm{PM}$ & 95 & 600 & 10 & 1.8 & 0.0 & 7 & 01:14 PM & 7.5 & 23.0 & 40 & \\
\hline 9 & $01.55 \mathrm{PM}$ & 95 & 600 & 10 & 1.8 & 0.0 & 8 & $02: 06 \mathrm{PM}$ & 7.5 & 23.0 & 37 & \\
\hline & & & & & & & & & & & & \\
\hline 10 & $02: 55 \mathrm{PM}$ & 95 & 600 & 10 & 1.8 & 0.0 & 9 & 03:05 PM & 7.5 & 24.0 & 42 & \\
\hline 11 & $03: 45 \mathrm{PM}$ & 95 & 600 & 10 & 1.8 & 0.0 & 10 & 04:00 PM & 7.5 & 24.0 & 33 & \\
\hline 12 & $04: 25 \mathrm{PM}$ & 95 & 600 & 10 & 1.8 & 0.0 & 11 & $04: 37 \mathrm{PM}$ & 7.5 & 24.0 & 33 & \\
\hline & & & & & & & & & & & & \\
\hline
\end{tabular}


ACT REMBCO JOINT VENTURE

Ornl Wag 4 Seeps 4 \& 6 Removal Action Project

Acrylamide Batching Record For

September 27, 1996

\begin{tabular}{|c|c|c|c|c|c|c|c|c|c|c|c|c|}
\hline \multirow{2}{*}{$\begin{array}{c}\text { Batch } \\
\text { No. }\end{array}$} & \multirow{2}{*}{$\begin{array}{l}\text { Time of } \\
\text { Batch }\end{array}$} & \multicolumn{3}{|c|}{ Tank A } & \multicolumn{2}{|c|}{ Tank $\mathrm{B}$} & \multirow{2}{*}{$\begin{array}{c}\text { Sample } \\
\text { No. }\end{array}$} & \multirow{2}{*}{$\begin{array}{l}\text { Time of } \\
\text { Sample }\end{array}$} & \multirow[t]{2}{*}{$\mathrm{pH}$} & \multirow{2}{*}{$\begin{array}{c}\text { Sample } \\
\text { Temp. } \\
\text { (Deg. C) }\end{array}$} & \multirow{2}{*}{$\begin{array}{c}\text { Gel } \\
\text { Time } \\
\text { (min) }\end{array}$} & \multirow[t]{2}{*}{ Comments } \\
\hline & & $\begin{array}{c}A V-100 \\
(L)\end{array}$ & $\begin{array}{c}\text { CAT-T+ } \\
(\mathrm{mL})\end{array}$ & $\begin{array}{l}\text { KFeCN } \\
\text { (grams) }\end{array}$ & $\begin{array}{c}\mathrm{AP} \\
(\mathrm{Kg})\end{array}$ & $\begin{array}{c}\text { Buffer } \\
\text { (Kg) }\end{array}$ & & & & & & \\
\hline 1 & & 95 & 600 & 10 & 1.8 & 0.0 & & & 7.5 & & & Remaining from yesterday \\
\hline & & & & & & & & & & & & \\
\hline 2 & $09: 05 \mathrm{AM}$ & 95 & 600 & 10 & 1.8 & 0.0 & 1 & 09:13 AM & 7.5 & 22.0 & 42 & \\
\hline 3 & $10: 45 \mathrm{AM}$ & 95 & 600 & 10 & 1.8 & 0.0 & 2 & $10: 52 \mathrm{AM}$ & 7.5 & 22.0 & 41 & \\
\hline & & & & & & & & & & & & \\
\hline 4 & $11: 30 \mathrm{AM}$ & 95 & 600 & 10 & $1 . \overline{8}$ & 0.0 & 3 & $11: 45 \mathrm{AM}$ & 7.5 & 22.0 & 33 & \\
\hline & & & & & & & & & & & & \\
\hline 5 & $12: 20 \mathrm{PM}$ & 95 & 600 & 10 & 1.8 & 0.0 & 4 & 12:25 PM & 7.5 & 22.0 & 35 & \\
\hline & & & & & & & & & & & & \\
\hline 6 & 01:00 PM & 95 & 600 & 10 & 1.8 & 0.0 & 5 & 01:08 PM & 7.5 & 22.0 & 35 & \\
\hline 7 & $01: 55 \mathrm{PM}$ & 95 & 600 & 10 & 1.8 & 0.0 & 6 & $01: 59 \mathrm{PM}$ & 7.5 & 22.0 & $\overline{36}$ & \\
\hline & & & & & & & & & & & & \\
\hline 8 & $02: 40 \mathrm{PM}$ & 95 & 600 & 10 & 1.8 & $0 . \overline{0}$ & 7 & $02: 48 \mathrm{PM}$ & 7.5 & 22.0 & 35 & \\
\hline & & & & & & & & & & & & \\
\hline
\end{tabular}

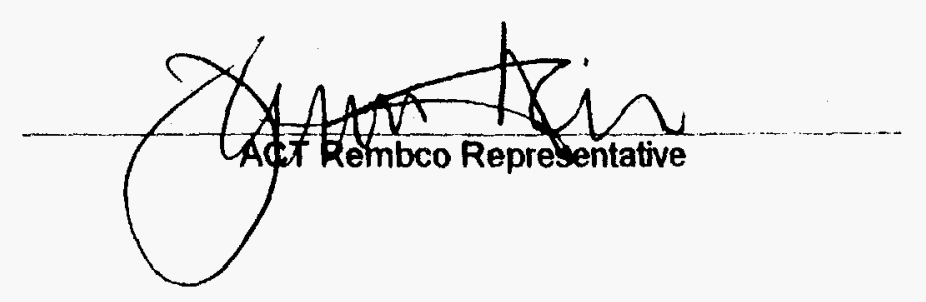




\section{ACT REMBCO JOINT VENTURE}

Ornl Wag 4 Seeps 4 \& 6 Removal Action Project

\section{Acrylamide Batching Record For}

September 30, 1996

\begin{tabular}{|c|c|c|c|c|c|c|c|c|c|c|c|c|}
\hline \multirow{2}{*}{$\begin{array}{l}\text { Batch } \\
\text { No. }\end{array}$} & \multirow{2}{*}{$\begin{array}{l}\text { Time of } \\
\text { Batch }\end{array}$} & \multicolumn{3}{|c|}{ Tank A } & \multicolumn{2}{|c|}{ Tank B } & \multirow{2}{*}{$\begin{array}{c}\text { Sample } \\
\text { No. }\end{array}$} & \multirow{2}{*}{$\begin{array}{l}\text { Time of } \\
\text { Sample }\end{array}$} & \multirow[t]{2}{*}{$\mathrm{pH}$} & \multirow{2}{*}{$\begin{array}{l}\text { Sample } \\
\text { Temp. } \\
\text { (Deg. C) }\end{array}$} & \multirow{2}{*}{$\begin{array}{c}\text { Gel } \\
\text { Time } \\
\text { (min) }\end{array}$} & \multirow[t]{2}{*}{ Comments } \\
\hline & & $\begin{array}{c}\text { AV-100 } \\
\text { (L) }\end{array}$ & $\begin{array}{c}\text { CAT-T+ } \\
(\mathrm{mL})\end{array}$ & $\begin{array}{l}\mathrm{KFeCN} \\
\text { (grams) }\end{array}$ & $\begin{array}{c}\text { AP } \\
(\mathrm{Kg})\end{array}$ & $\begin{array}{c}\text { Buffer } \\
(\mathrm{Kg})\end{array}$ & & & & & & \\
\hline 1 & & 95 & 600 & 10 & 1.8 & 0.0 & & & 7.5 & & & Remaining from yesterday \\
\hline 2 & & 95 & 600 & 10 & 1.8 & 0.0 & 1 & $08: 43 \mathrm{AM}$ & $\overline{7.5}$ & 16.0 & 54 & Remaining from yesterday \\
\hline 3 & $09.50 \mathrm{AM}$ & 95 & 600 & 10 & 1.8 & 0.0 & 2 & $09: 56 \mathrm{AM}$ & 7.5 & 15.0 & 70 & \\
\hline & & & & & & & & & & & & \\
\hline 4 & $11: 00 A \bar{M}$ & 95 & 600 & 10 & 1.8 & 0.0 & 3 & 12:44 PM & 7.5 & 18.0 & 48 & \\
\hline 5 & $12: 05 \mathrm{PM}$ & 95 & 600 & 10 & 1.8 & 0.0 & 4 & 12:14 PM & 7.5 & 18.0 & 55 & \\
\hline & & & & & & & & & & & & \\
\hline 6 & $01: 05 \mathrm{PM}$ & 95 & 600 & 10 & 1.8 & 0.0 & 5 & $01: 14 \mathrm{PM}$ & 7.5 & 18.0 & 48 & \\
\hline$\overline{7}$ & 02:10 PM & 95 & 600 & 10 & 1.8 & 0.0 & 6 & 02:22 PM & $\overline{7.5}$ & 22.0 & 41 & \\
\hline & & & & & & & & & & & & \\
\hline
\end{tabular}

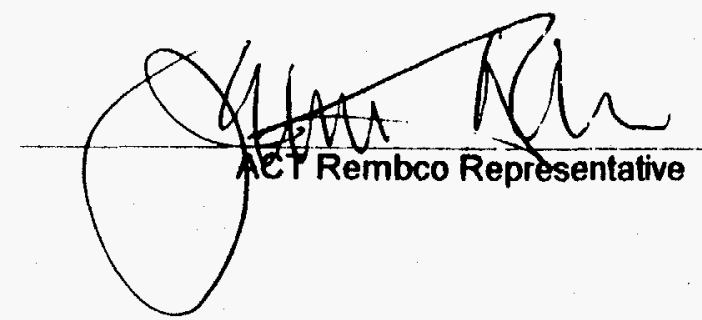


ACT REMBCO JOINT VENTURE

Ornl Wag 4 Seeps 4 \& 6 Removal Action Project

Acrylamide Batching Record For

October 01, 1996

\begin{tabular}{|c|c|c|c|c|c|c|c|c|c|c|c|c|}
\hline \multirow{2}{*}{$\begin{array}{l}\text { Batch } \\
\text { No. }\end{array}$} & \multirow{2}{*}{$\begin{array}{l}\text { Time of } \\
\text { Batch }\end{array}$} & \multicolumn{3}{|c|}{ Tank A } & \multicolumn{2}{|c|}{ Tank $\bar{B}$} & \multirow{2}{*}{$\begin{array}{c}\text { Sample } \\
\text { No. }\end{array}$} & \multirow{2}{*}{$\begin{array}{l}\text { Time of } \\
\text { Sample }\end{array}$} & \multirow[t]{2}{*}{$\mathrm{pH}$} & \multirow{2}{*}{$\begin{array}{l}\text { Sample } \\
\text { Temp. } \\
\text { (Deg. C) }\end{array}$} & \multirow{2}{*}{$\begin{array}{l}\text { Gel } \\
\text { Time } \\
\text { (min) }\end{array}$} & \multirow[t]{2}{*}{ Comments } \\
\hline & & $\begin{array}{l}A V-100 \\
\text { (L) }\end{array}$ & $\begin{array}{c}\text { CAT-T+ } \\
(\mathrm{mL})\end{array}$ & $\begin{array}{l}\text { KFeCN } \\
\text { (grams) }\end{array}$ & $\begin{array}{l}\text { AP } \\
(\mathrm{Kg})\end{array}$ & $\begin{array}{c}\text { Buffer } \\
(\mathrm{Kg})\end{array}$ & & & & & & \\
\hline 1 & & 95 & 600 & 10 & 1.8 & 0.0 & & & $7 . \overline{5}$ & & & Remaining from yesterday \\
\hline 5 & & 05 & 600 & 10 & 18 & 00 & 1 & $07 \cdot 10 \mathrm{AM}$ & 75 & 200 & AM & Pomainin from vactardav \\
\hline & & 90 & & 10 & 1.0 & 0.0 & 1 & $07.49 \mathrm{AM}$ & & 20.0 & 44 & Remaiming woon yesteruay \\
\hline 3 & $08.25 \mathrm{AM}$ & 95 & 600 & 10 & 1.8 & 0.0 & 2 & $08: 35 \mathrm{AM}$ & 7.5 & 20.0 & 38 & \\
\hline 4 & $09: 25 \mathrm{AM}$ & 95 & 600 & 10 & 1.8 & 0.0 & 3 & $09: 35 \mathrm{AM}$ & 7.5 & 20.0 & 37 & \\
\hline 5 & $10: 15 \mathrm{AM}$ & 95 & 600 & 10 & 1.8 & 0.0 & 4 & $10: 33 \mathrm{AM}$ & 7.5 & $20.0^{2}$ & 40 & \\
\hline & & & & & & & & & & & & \\
\hline 6 & $11: 00 \mathrm{AM}$ & 95 & 600 & 10 & 1.8 & 0.0 & 5 & $11: 10 \mathrm{AM}$ & 7.5 & 20.0 & 44 & \\
\hline$\overline{7}$ & $11: 55 \mathrm{AM}$ & $\overline{9}$ & 600 & 10 & 1.8 & $\overline{0.0}$ & 6 & $12: 14 \mathrm{PM}$ & 7.5 & 20.0 & 36 & \\
\hline 8 & $01: 00 \mathrm{PM}$ & 95 & 600 & 10 & 1.8 & 0.0 & 7 & $01: 09 \mathrm{PM}$ & 7.5 & 21.0 & 48 & \\
\hline & & & & & & & & & & & & \\
\hline 9 & $01: 50 \mathrm{PM}$ & 95 & 600 & 10 & 1.8 & 0.0 & 8 & 02:05 PM & 7.5 & 21.0 & 35 & \\
\hline 10 & $02: 35 \mathrm{PM}$ & 95 & 600 & 10 & 1.8 & 0.0 & 9 & $\overline{02: 44 \mathrm{PM}}$ & 7.5 & 20.0 & 38 & \\
\hline & & & & & & & & & & & & \\
\hline
\end{tabular}




\section{ACT REMBCO JOINT VENTURE}

Ornl Wag 4 Seeps 4 \& 6 Removal Action Project

Acrylamide Batching Record For October 02, 1996

\begin{tabular}{|c|c|c|c|c|c|c|c|c|c|c|c|c|}
\hline $\begin{array}{c}\text { Batch } \\
\text { No. }\end{array}$ & $\begin{array}{l}\text { Time of } \\
\text { Batch }\end{array}$ & $\begin{array}{c}\overline{A V}-10 \overline{0} \\
\text { (L) }\end{array}$ & $\begin{array}{c}\text { Tank A } \\
\text { CAT-T+ } \\
(\mathrm{mL})\end{array}$ & $\begin{array}{l}\mathrm{KFeCN} \\
\text { (grams) }\end{array}$ & $\begin{array}{c}\mathrm{AP} \\
(\mathrm{Kg})\end{array}$ & $\begin{array}{c}\bar{B} \\
\text { Buffer }\end{array}$ & $\begin{array}{c}\text { Sample } \\
\text { No. }\end{array}$ & $\begin{array}{l}\text { Time of } \\
\text { Sample }\end{array}$ & $\mathrm{pH}$ & $\begin{array}{c}\text { Sample } \\
\text { Temp. } \\
\text { (Deg. C) }\end{array}$ & $\begin{array}{c}\text { Gel } \\
\text { Time } \\
\text { (min) }\end{array}$ & Comments \\
\hline 1 & & 95 & 600 & 10 & 1.8 & 0.0 & & & 7.5 & & & Remaining from yesterday \\
\hline$\overline{2}$ & & 95 & 600 & 10 & 1.8 & 0.0 & & & 7.5 & & & Remaining from yesterday \\
\hline 3 & $09.55 \mathrm{AM}$ & 95 & 600 & 10 & 1.8 & 0.0 & 1 & 10:07 AM & 7.5 & 20.0 & 38 & \\
\hline-4 & $10: 15 \mathrm{AM}$ & 95 & 600 & 10 & 1.8 & 0.0 & 2 & $10: 27 \mathrm{AM}$ & 7.5 & 20.0 & 25 & \\
\hline 5 & $11: 25 \mathrm{AM}$ & 95 & 600 & 10 & 1.8 & 0.0 & 3 & $11: 40 \mathrm{AM}$ & 7.5 & 21.0 & 37 & \\
\hline 6 & $11: 45 \mathrm{AM}$ & 95 & 600 & 10 & 1.8 & 0.0 & 4 & 11:50 AM & 7.5 & 22.0 & 48 & \\
\hline 7 & $01: 45 \mathrm{PM}$ & 95 & 600 & 10 & 1.8 & 0.0 & 5 & 02:08 PM & 7.5 & 22.0 & 35 & \\
\hline 8 & $02: \overline{4} \overline{P M}$ & 95 & 600 & 10 & $1 . \overline{8}$ & 0.0 & 6 & $02: 53 \mathrm{PM}$ & 7.5 & 21.0 & 39 & \\
\hline 9 & 03:05 PM & 95 & 600 & 10 & 1.8 & 0.0 & 7 & 03:13 PM & 7.5 & 21.0 & 40 & \\
\hline 10 & $04: 10 \mathrm{PM}$ & 95 & 600 & 10 & 1.8 & 0.0 & 8 & 04:27 PM & 7.5 & 22.0 & 35 & \\
\hline 11 & $04: 40 \mathrm{PM}$ & 95 & 600 & 10 & 1.8 & 0.0 & 9 & $04: 45 \mathrm{PM}$ & 7.5 & 22.0 & 35 & \\
\hline
\end{tabular}


ACT REMBCO JOINT VENTURE

Ornl Wag 4 Seeps 4 \& 6 Removal Action Project

Acrylamide Batching Record For

October 03, 1996

\begin{tabular}{|c|c|c|c|c|c|c|c|c|c|c|c|c|}
\hline \multirow{2}{*}{$\begin{array}{l}\text { Batch } \\
\text { No. }\end{array}$} & \multirow{2}{*}{$\begin{array}{l}\text { Time of } \\
\text { Batch }\end{array}$} & \multicolumn{3}{|c|}{ Tank A } & \multicolumn{2}{|c|}{ Tank B } & \multirow{2}{*}{$\begin{array}{c}\text { Sample } \\
\text { No. }\end{array}$} & \multirow{2}{*}{$\begin{array}{l}\text { Time of } \\
\text { Sample }\end{array}$} & \multirow[t]{2}{*}{$\mathrm{pH}$} & \multirow{2}{*}{$\begin{array}{l}\text { Sample } \\
\text { Temp. } \\
\text { (Deg. C) }\end{array}$} & \multirow{2}{*}[\begin{array}{c}{\text{Gel}}\\
{\text{Time}}\\
{\text{(min)}}\end{array}]{} & \multirow[t]{2}{*}{ Comments } \\
\hline & & $\begin{array}{c}A V-100 \\
\text { (L) }\end{array}$ & $\begin{array}{c}\text { CAT-T+ } \\
(\mathrm{mL})\end{array}$ & $\begin{array}{l}\text { KFeCN } \\
\text { (grams) }\end{array}$ & $\begin{array}{c}\mathrm{AP} \\
(\mathrm{Kg})\end{array}$ & $\begin{array}{c}\text { Buffer } \\
\text { (Kg) }\end{array}$ & & & & & & \\
\hline 1 & & 95 & 600 & 10 & 1.8 & 0.0 & & & 7.5 & & & Remaining from yesterday \\
\hline & & & & & & & & & & & & \\
\hline 2 & $07: 35 \mathrm{AM}$ & 95 & 600 & 10 & 1.8 & 0.0 & 1 & 07:50 AM & 7.5 & 21.0 & 35 & \\
\hline 3 & $08: 45 \mathrm{AM}^{-}$ & 95 & 600 & 10 & 1.8 & 0.0 & 2 & $09: 06 \mathrm{AM}$ & 7.5 & 21.0 & 34 & \\
\hline & & & & & & & & & & & & \\
\hline 4 & $09: 40 \mathrm{AM}$ & $9 \overline{5}$ & 600 & 10 & 1.8 & 0.0 & 3 & 09:50 AM & 7.5 & 21.0 & 39 & \\
\hline & & & & & & & & & & & & \\
\hline 5 & $10: 20 \mathrm{AM}$ & 95 & 600 & 10 & 1.8 & 0.0 & 4 & 10:28 AM & 7.5 & 21.0 & 34 & \\
\hline 6 & $11: 05 \mathrm{AM}$ & 95 & 600 & 10 & 1.8 & 0.0 & 5 & $11: 08 \mathrm{AM}$ & 7.5 & 21.0 & 40 & \\
\hline & & & & & & & & & & & & \\
\hline 7 & $11: \overline{35} \overline{\mathrm{AM}}$ & 95 & 600 & 10 & 1.8 & 0.0 & 6 & $11: 46 \mathrm{AM}$ & 7.5 & 22.0 & 25 & \\
\hline 8 & $12: 00 \mathrm{PM}$ & 95 & 600 & 10 & 1.8 & 0.0 & 7 & $12: 12 \mathrm{PM}$ & 7.5 & 22.0 & 36 & \\
\hline & & & & & & & & & & & & \\
\hline 9 & $12: 40 \mathrm{PM}$ & 95 & 600 & 10 & 1.8 & 0.0 & 8 & 01:05 PM & 7.5 & 22.0 & 35 & \\
\hline & $01.15 \mathrm{PM}$ & 05 & 600 & 10 & 19 & n & 9 & n1.22 PM & 75 & 220 & 35 & \\
\hline 10 & U1.15 MM & 95 & 600 & 10 & 1.8 & 0.0 & $y$ & $01.2 \angle 2+11$ & & 22.0 & & \\
\hline 11 & $01: 45 \mathrm{PM}$ & 95 & 600 & 10 & 1.8 & 0.0 & 10 & $01: 55 \mathrm{PM}$ & 7.5 & 23.0 & 40 & \\
\hline & & & & & & & & & & & & \\
\hline
\end{tabular}

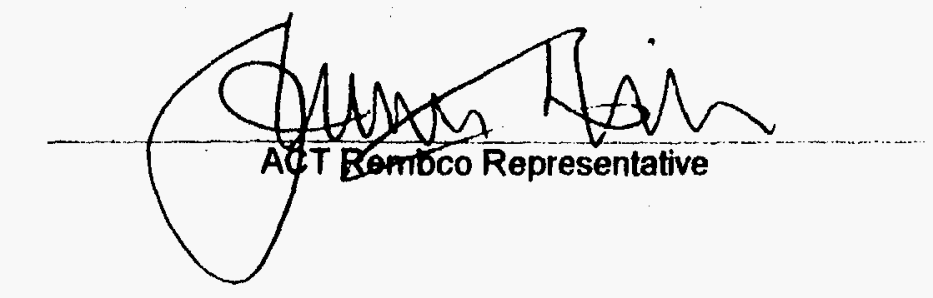




\section{ACT REMBCO JOINT VENTURE}

Ornl Wag 4 Seeps 4 \& 6 Removal Action Project

Acrylamide Batching Record For

October 08, 1996

\begin{tabular}{|c|c|c|c|c|c|c|c|c|c|c|c|c|}
\hline \multirow{2}{*}{$\begin{array}{l}\text { Batch } \\
\text { No. }\end{array}$} & \multirow{2}{*}{$\begin{array}{l}\text { Time of } \\
\text { Batch }\end{array}$} & \multicolumn{3}{|c|}{ Tank A } & \multicolumn{2}{|c|}{ Tank B } & \multirow{2}{*}{$\begin{array}{c}\text { Sample } \\
\text { No. }\end{array}$} & \multirow{2}{*}{$\begin{array}{l}\text { Time of } \\
\text { Sample }\end{array}$} & \multirow[t]{2}{*}{$\mathrm{pH}$} & \multirow{2}{*}{$\begin{array}{l}\text { Sample } \\
\text { Temp. } \\
\text { (Deg. C) }\end{array}$} & \multirow{2}{*}{$\begin{array}{c}\text { Gel } \\
\text { Time } \\
\text { (min) }\end{array}$} & \multirow{2}{*}{ Comments } \\
\hline & & $\begin{array}{c}A V-100 \\
\text { (L) }\end{array}$ & $\begin{array}{c}\text { CAT-T+ } \\
(\mathrm{mL})\end{array}$ & $\begin{array}{l}\text { KFeCN } \\
\text { (grams) }\end{array}$ & $\begin{array}{l}\overline{A P} \\
(\mathrm{Kg})\end{array}$ & $\begin{array}{c}\text { Buffer } \\
(\mathrm{Kg})\end{array}$ & & & & & & \\
\hline 1 & & 95 & 600 & 10 & 1.8 & 0.0 & & & 7.5 & & & Remaining from yesterday \\
\hline 2 & & 95 & 600 & 10 & 18 & 00 & 1 & $07.55 \mathrm{AM}$ & 75 & 160 & 75 & Remaining from vesterdav \\
\hline & & & & & & & & & & & & \\
\hline 3 & $\overline{08}: \overline{55} \mathrm{AM}$ & 95 & 600 & 10 & 1.8 & 0.0 & 2 & $\overline{09: 00 ~ A M}$ & 7.5 & 18.0 & 37 & \\
\hline$\pi$ & $00.45 \mathrm{AME}$ & 05 & 900 & $\overline{5}$ & 10 & 0 & 3 & $00.25 \mathrm{AM}$ & 75 & $100+2+253$ & 22 & \\
\hline & $09.15 \mathrm{AlM}$ & & out & & 1.0 & 0.0 & & $00.20 \mathrm{ANW}$ & 1.0 & & & \\
\hline 5 & $09: 45 \mathrm{AM}$ & 95 & 600 & 5 & 1.8 & 0.0 & 4 & 09:57 AM & 7.5 & 20.0 & 28 & \\
\hline$\overline{6}$ & $10: 15$ AM & $\overline{95}$ & 600 & 5 & 1.8 & 0.0 & 5 & $10.24 \mathrm{AM}$ & $\overline{7.5}$ & 20.0 & 22 & \\
\hline & & & & & & & & & & & & \\
\hline$\overline{7}$ & $11: 10 \mathrm{AM}$ & $\overline{95}$ & 600 & 5 & 1.8 & 0.0 & 6 & $11: 24 \mathrm{AM}$ & 7.5 & 20.0 & 22 & \\
\hline 8 & $11: 40 \mathrm{AM}$ & 95 & 600 & 5 & 1.8 & 0.0 & 7 & $11: 54 \mathrm{AM}$ & $\overline{7.5}$ & 20.0 & 21 & \\
\hline & & & & & & & & & & & & \\
\hline
\end{tabular}


ACT REMBCO JOINT VENTURE

Ornl Wag 4 Seeps 4 \& 6 Removal Action Project

Acrylamide Batching Record For

October 11, 1996

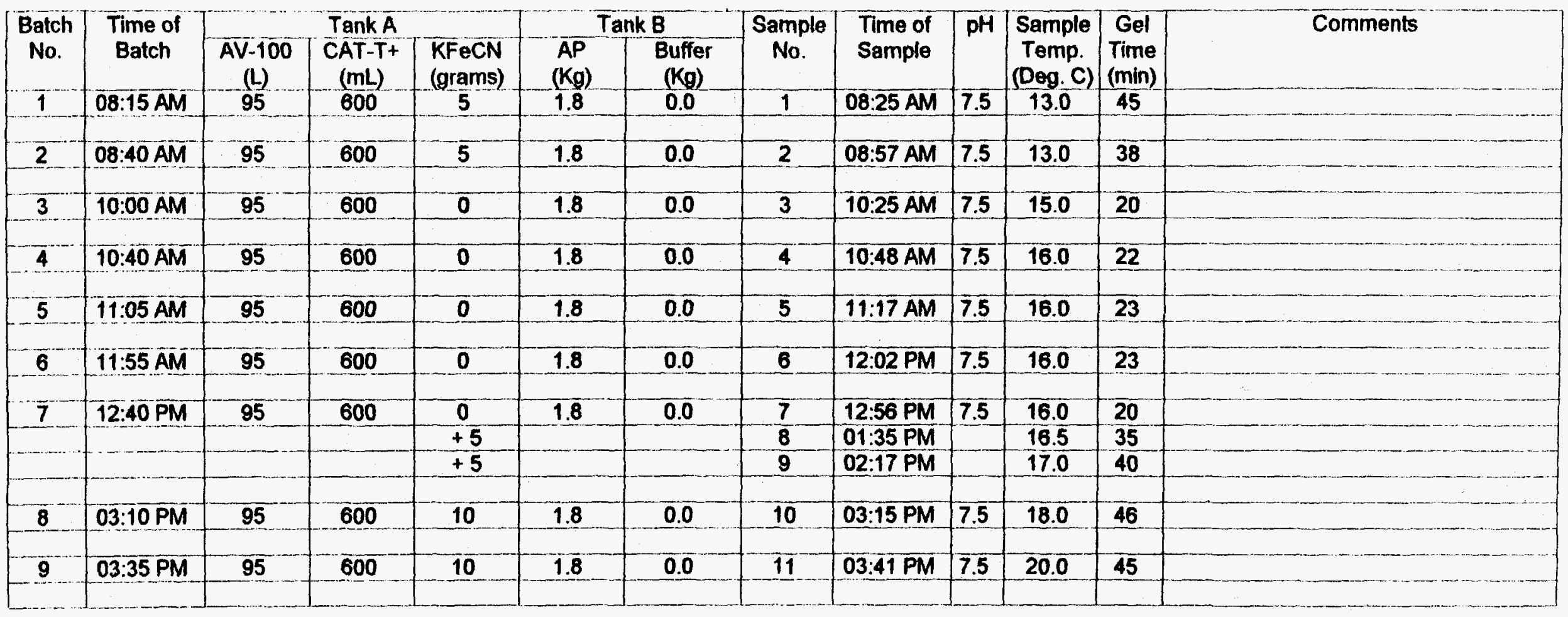

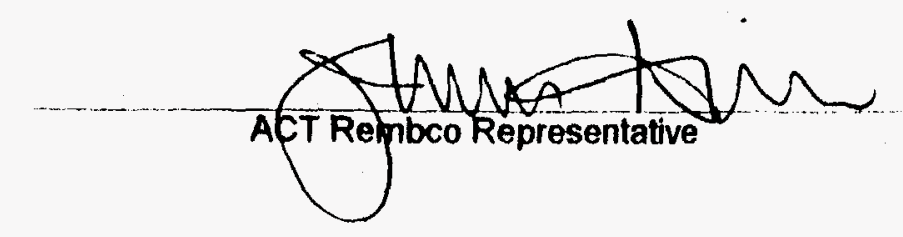


ACT REMBCO JOINT VENTURE

Ornil Wag 4 Seeps 4 \& 6 Removal Action Project

Acrylamide Batching Record For

October 12, 1996

\begin{tabular}{|c|c|c|c|c|c|c|c|c|c|c|c|c|}
\hline \multirow{2}{*}{$\begin{array}{l}\text { Batch } \\
\text { No. }\end{array}$} & \multirow{2}{*}{$\begin{array}{l}\text { Time of } \\
\text { Batch }\end{array}$} & \multicolumn{3}{|c|}{ Tank $\bar{A}$} & \multicolumn{2}{|c|}{ Tank B } & \multirow{2}{*}{$\begin{array}{c}\text { Sample } \\
\text { No. }\end{array}$} & \multirow{2}{*}{$\begin{array}{l}\text { Time of } \\
\text { Sample }\end{array}$} & \multirow[t]{2}{*}{ pH } & \multirow{2}{*}{$\begin{array}{c}\text { Sample } \\
\text { Temp. } \\
\text { (Deg. C) }\end{array}$} & \multirow{2}{*}{$\begin{array}{l}\text { Gel } \\
\text { Time } \\
\text { (min) }\end{array}$} & \multirow{2}{*}{ Comments } \\
\hline & & $\begin{array}{l}A V-100 \\
\text { (L) }\end{array}$ & $\begin{array}{c}\text { CAT-T+ } \\
(\mathrm{mL})\end{array}$ & $\begin{array}{l}\mathrm{KFeCN} \\
\text { (grams) }\end{array}$ & $\begin{array}{c}\mathrm{AP} \\
(\mathrm{Kg})\end{array}$ & $\begin{array}{c}\text { Buffer } \\
(\mathrm{Kg})\end{array}$ & & & & & & \\
\hline 1 & & 95 & 600 & 10 & 1.8 & 0.0 & & & 7.5 & & & Remaining from yesterday \\
\hline 2 & $08: 10 \mathrm{AM}$ & 95 & 600 & 5 & 1.8 & 0.0 & 1 & $08: 20 \mathrm{AM}$ & 7.5 & 13.0 & 50 & \\
\hline 3 & $08: 45 \overline{A M}$ & $\overline{95}$ & 600 & 5 & 1.8 & 0.0 & 2 & $\overline{08: 50} \overline{A M}$ & 7.5 & 15.0 & 43 & \\
\hline 4 & $09: 20 \mathrm{AM}$ & $\overline{95}$ & 600 & 5 & 18 & 00 & 3 & $09 \cdot 29 \mathrm{AM}$ & 75 & 150 & 15 & \\
\hline & & & & & & & & & & & & \\
\hline 5 & $09: 50 \mathrm{AM}$ & 95 & 600 & 5 & 1.8 & 0.0 & 4 & 10:02 AM & 7.5 & 16.0 & 43 & \\
\hline 6 & $10: 20 \mathrm{AM}$ & $\overline{95}$ & 600 & 5 & 1.8 & 0.0 & 5 & $10.34 \mathrm{AM}$ & 75 & 170 & 48 & \\
\hline & & & & & & & & & & & & \\
\hline 7 & $10: 50 \mathrm{AM}$ & 95 & 600 & 5 & 1.8 & 0.0 & 6 & 11:10 AM & 7.5 & 17.0 & 48 & \\
\hline 8 & $11: 25 \overline{A M}$ & 95 & 600 & 5 & 1.8 & 0.0 & 7 & $11: 37 \mathrm{AM}$ & 7.5 & $17 . \overline{0}$ & 50 & \\
\hline & & & & & & & & & & & & \\
\hline 9 & $12: 00 \mathrm{PM}$ & 95 & 600 & 5 & 1.8 & 0.0 & 8 & 12:07 PM & 7.5 & 18.0 & 45 & \\
\hline 10 & $12: 45 \mathrm{PM}$ & $\overline{95}$ & 600 & 5 & 1.8 & 0.0 & 9 & 12:52 PM & 7.5 & 18.0 & 43 & \\
\hline 11 & $01: 10 \mathrm{PM}$ & 95 & 600 & 10 & 18 & 00 & 10 & $01.18 \mathrm{DM}$ & 75 & 180 & CE & \\
\hline & & & & 10 & & 0.0 & 10 & n & 1.2 & 18.0 & 65 & \\
\hline 12 & 01:35 PM & 95 & 600 & 10 & 1.8 & 0.0 & 11 & $01: 55 \mathrm{PM}$ & 7.5 & 18.0 & 60 & \\
\hline & & & & & & & & & & & & \\
\hline
\end{tabular}


ACT REMBCO JOINT VENTURE

Oml Wag 4 Seeps 4 \& 6 Removal Action Project

Acrylamide Batching Record For

October 18, 1996

\begin{tabular}{|c|c|c|c|c|c|c|c|c|c|c|c|c|}
\hline \multirow{2}{*}{$\begin{array}{l}\text { Batch } \\
\text { No. }\end{array}$} & \multirow{2}{*}{$\begin{array}{l}\text { Time of } \\
\text { Batch }\end{array}$} & \multicolumn{3}{|c|}{ Tank A } & \multicolumn{2}{|c|}{ Tank B } & \multirow{2}{*}{$\begin{array}{c}\text { Sample } \\
\text { No. }\end{array}$} & \multirow{2}{*}{$\begin{array}{l}\text { Time of } \\
\text { Sample }\end{array}$} & \multirow[t]{2}{*}{$\mathrm{pH}$} & \multirow{2}{*}{$\begin{array}{c}\text { Sample } \\
\text { Temp. } \\
\text { (Deg. C) }\end{array}$} & \multirow{2}{*}{$\begin{array}{c}\text { Gel } \\
\text { Time } \\
\text { (min) }\end{array}$} & \multirow[t]{2}{*}{ Comments } \\
\hline & & $\begin{array}{c}A V-100 \\
\text { (L) }\end{array}$ & $\begin{array}{c}\text { CAT-T+ } \\
(\mathrm{mL})\end{array}$ & $\begin{array}{l}\text { KFeCN } \\
\text { (grams) }\end{array}$ & $\begin{array}{c}\mathrm{AP} \\
(\mathrm{Kg})\end{array}$ & $\begin{array}{l}\text { Buffer } \\
(\mathrm{Kg})\end{array}$ & & & & & & \\
\hline 1 & & 95 & 600 & 10 & 1.8 & 0.0 & & & 7.5 & & & Remaining from yesterday \\
\hline 2 & 08:02 AM & 95 & 600 & 10 & 1.8 & 0.0 & 1 & 08:03 AM & 7.5 & 20.0 & 53 & \\
\hline 3 & $09: 30 \mathrm{AM}$ & $\overline{95}$ & 600 & 10 & 1.8 & 0.0 & 2 & 09:32 AM & 7.5 & 20.0 & 60 & \\
\hline 4 & $10: 05 \mathrm{AM}$ & $9 \overline{5}$ & 600 & 10 & 1.8 & 0.0 & 3 & 10:06 AM & 7.5 & 20.0 & 62 & \\
\hline 5 & $10: 49 \overline{A M}$ & 95 & 600 & 10 & 1.8 & 0.0 & 4 & $10: 50$ AM & 7.5 & 18.0 & 68 & \\
\hline 6 & $11: 37 \overline{A M}$ & 95 & 600 & 10 & 1.8 & 0.0 & 5 & $11: 38$ AM & 7.5 & 20.0 & 68 & \\
\hline 7 & $12: 43 \mathrm{PM}$ & $9 \overline{5}$ & 600 & 10 & 1.8 & 0.0 & 6 & $12: 44 \mathrm{PM}$ & 7.5 & 15.0 & 70 & \\
\hline 8 & $01: 20 \mathrm{PM}$ & 95 & 600 & 5 & 1.8 & 0.0 & 7 & $01: 47$ PM & 7.5 & 18.0 & & \\
\hline$\overline{9}$ & $02: 13 \mathrm{PM}$ & 95 & 600 & 5 & 1.8 & 0.0 & 8 & 02:14 PM & 7.5 & 18.0 & 70 & \\
\hline 10 & $03: 36 \mathrm{PM}$ & 95 & 600 & 5 & 1.8 & 0.0 & 9 & 03:37 PM & 7.5 & 18.0 & 43 & \\
\hline 11 & $04: 17 \mathrm{PM}$ & 95 & 600 & 5 & 1.8 & 0.0 & 10 & 04:19 PM & 7.5 & 19.0 & & \\
\hline
\end{tabular}

Thbnat

ACT Rembco Representative 
ACT REMBCO JOINT VENTURE

Ornl Wag 4 Seeps 4 \& 6 Removal Action Project

Acrylamide Batching Record For

October 21, 1996

\begin{tabular}{|c|c|c|c|c|c|c|c|c|c|c|c|c|}
\hline \multirow{2}{*}{$\begin{array}{l}\text { Batch } \\
\text { No. }\end{array}$} & \multirow{2}{*}{$\begin{array}{l}\text { Time of } \\
\text { Batch }\end{array}$} & \multicolumn{3}{|c|}{ Tank A } & \multicolumn{2}{|c|}{ Tank B } & \multirow{2}{*}{$\begin{array}{c}\text { Sample } \\
\text { No. }\end{array}$} & \multirow{2}{*}{$\begin{array}{l}\text { Time of } \\
\text { Sample }\end{array}$} & \multirow{2}{*}{$\mathrm{pH}$} & \multirow{2}{*}{$\begin{array}{c}\text { Sample } \\
\text { Temp. } \\
\text { (Deg. C) }\end{array}$} & \multirow{2}{*}{$\begin{array}{c}\text { Gel } \\
\text { Time } \\
\text { (min) }\end{array}$} & \multirow{2}{*}{ Comments } \\
\hline & & $\begin{array}{c}A V-100 \\
(L)\end{array}$ & $\begin{array}{c}\text { CAT-T+ } \\
(\mathrm{mL})\end{array}$ & $\begin{array}{l}\text { KFeCN } \\
\text { (grams) }\end{array}$ & $\begin{array}{l}\mathrm{AP} \\
(\mathrm{Kg})\end{array}$ & $\begin{array}{l}\text { Buffer } \\
(\mathrm{Kg})\end{array}$ & & & & & & \\
\hline 1 & & 95 & 600 & 10 & 1.8 & 0.0 & & & 7.5 & & & Remaining from yesterday \\
\hline 2 & 09:00 AM & 95 & 600 & 5 & 1.8 & 0.0 & 1 & 09:02 AM & 7.5 & 10.0 & 65 & \\
\hline & & & & & & & & & & & & \\
\hline 3 & 09:48 AM & 95 & 600 & 5 & 1.8 & 0.0 & 2 & $09: 49 \mathrm{AM}$ & 7.5 & 11.0 & 72 & \\
\hline 4 & 10:20 AM & 95 & 600 & 5 & 1.8 & 0.0 & 3 & $10: 21 \mathrm{AM}$ & 7.5 & 12.0 & 66 & \\
\hline & & & & & & & & & & & & \\
\hline 5 & & 95 & 600 & 5 & 1.8 & 0.0 & & & & & & \\
\hline 6 & & 95 & 600 & 5 & 1.8 & 0.0 & & & & & & \\
\hline & & & & & & & & & & & & \\
\hline 7 & $12: 37 \mathrm{PM}$ & $9 \overline{5}$ & 600 & 5 & 1.8 & 0.0 & 4 & 12:39 PM & 7.5 & 13.0 & 64 & \\
\hline 8 & $01: 10 \mathrm{PM}$ & 95 & 600 & 5 & 1.8 & $\overline{0} . \overline{0}$ & 5 & 01:12 PM & 7.5 & 14.0 & 70 & \\
\hline$\overline{9}$ & 02:10 PM & 95 & 600 & 5 & 1.8 & 0.0 & 6 & $02: 24 \mathrm{PM}$ & 7.5 & 15.0 & 63 & t. \\
\hline & & & & & & & & & & & & \\
\hline 10 & 02:38 PM & 95 & 600 & 5 & 1.8 & 0.0 & 7 & $02: 39 \mathrm{PM}$ & 7.5 & 16.0 & 61 & \\
\hline & & & & & & & & & & & & \\
\hline
\end{tabular}

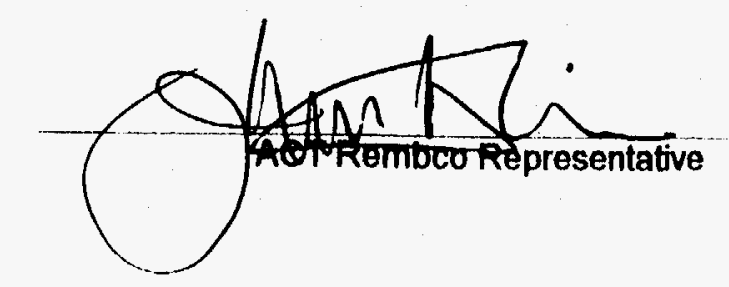




\section{DISTRIBUTION}

1. L. V. Asplund

2. W. D. Brickeen

3. D. D. Huff

4. T. M. Koepp

5. S. L. Laman

6-7. J. D. Long

8. P. T. Owen

9. L. B. Raulston

10. Central Research Library

11. File-EMEF DMC-RC

12-14. A. Naudts, ECO Grouting Specialists Limited, Cheltenham, Ontario, Canada

15. R. Boyd, International Technology Corporation, 312 Directors Drive, Knoxville, TN 37923

16. R. Berry, Rembco Engineering Corporation, 10001 Kingston Pike, Knoxville, TN 37922

17. J. Cockburn, Advanced Construction Techniques Limited, 10495 Keele St., Maple, Ontario, Canada L6A1R7 